UNIVERSIDAD POLITÉCNICA DE MADRID

ESCUELA TÉCNICA SUPERIOR DE INGENIEROS INDUSTRIALES

\title{
DESARROLLO DE UN MODELO DE EVALUACIÓN DE PAISAJES SONOROS SEGÚN ASPECTOS ESPACIALES, TEMPORALES, SUBJETIVOS Y DE CONTEXTO
}

\section{TESIS DOCTORAL}

Autor: Luis Fernando Hermida Cadena

Máster Universitario en Ingeniería Acústica en la Industria y el Transporte por la Universidad Politécnica de Madrid

Director: Ignacio Pavón García

Doctor por la Universidad Politécnica de Madrid 

Tribunal nombrado por el Mgfco. y Excmo. Sr. Rector de la Universidad Politécnica de Madrid, el día , de de 2019.

Presidente

Vocal

Vocal

Vocal

Secretario
D.

D.

D.

D.

D.

Realizado el acto de defensa y lectura de la tesis el día de de 2019.

Calificación:

El Presidente

El secretario

Los vocales 



\section{AGRADECIMIENTOS}

A la vida por ofrecerme tantas puertas abiertas a las cuales entrar.

A mi esposa Laura, por su amor y apoyo incondicional en el propósito de alcanzar sueños.

A mi familia por creer en mi y por su invaluable compañía.

Al Profesor Ignacio Pavón García, quien me ha guiado en estos años y me ha ofrecido de forma generosa e incondicional su conocimiento y apoyo.

Al Profesor Manuel Recuero por acogerme en este proceso de formación académica y ofrecerme la posibilidad de formarme bajo los más altos niveles de calidad en la Universidad Politécnica de Madrid.

Al Profesor Luis J. Bento Coelho por permitirme compartir con él y su grupo de investigación en el Instituto Superior Técnico de la Universidad de Lisboa en el tramo crucial de esta investigación.

A mi amigo Antonio Carlos Lobo Soares, compañero de dudas e inquietudes.

A la Profesora Fátima Aparecida dos Santos por presentarme el camino al estudio de la ciudad.

A todos los profesores y amigos que han aportado en este largo camino.

A la Universidad de San Buenaventura por apoyarme en este proceso de formación. 



\section{RESUMEN}

Esta investigación aborda la evaluación de paisajes sonoros desde aspectos objetivos, subjetivos y descriptivos. Para tal fin fueron analizados trece paisajes sonoros urbanos de las ciudades de Bogotá (Colombia), Brasilia (Brasil) y Lisboa (Portugal).

La primera parte de esta investigación se enfocó en la búsqueda de parámetros acústicos que permitieran describir atributos alternativos al confort y molestia frente al ruido, teniendo en cuenta comportamientos espaciales y temporales de las fuentes sonoras. En esta etapa fueron adaptados y aplicados al estudio de entornos acústicos parámetros derivados de las funciones de autocorrelación y correlación cruzada interaural (ACF e IACF) en sus modalidades móviles y de largo plazo. Así mismo, fueron desarrolladas pruebas de evaluación subjetiva y una serie de análisis descriptivos. Se obtuvieron altas correlaciones entre parámetros objetivos y atributos perceptuales, permitiendo apreciar que las variaciones de las fuentes sonoras en el plano horizontal y las variaciones energéticas temporales están asociadas a entornos variables, agitados o monótonos. Además, los resultados obtenidos de pruebas estadísticas indican diferencias estadísticamente significativas entre los entornos acústicos según parámetros acústicos derivados de las ACF e IACF y su sonido fundamental.

La segunda parte de la investigación indagó acerca de la influencia de aspectos contextuales y semióticos en la evaluación, diseño y gestión de paisajes sonoros, a partir de la comparación de metodologías de evaluación in situ y en laboratorio y del estudio del componente interacción lugar-persona. En lo que respecta a la comparación de metodologías de evaluación, se realizaron pruebas in situ y en laboratorio correspondientes a tres parques urbanos de la ciudad de Lisboa, encontrando diferencias estadísticamente significativas según el tipo de metodología usada. En relación a la influencia de la interacción lugar-persona en la evaluación de paisajes sonoros, fueron desarrolladas pruebas en laboratorio en las ciudades de Bogotá y Lisboa, donde se presentaron a los oyentes de cada ciudad fragmentos de audios binaurales de entornos acústicos de lugares conocidos y desconocidos. No fueron encontradas asociaciones considerables entre el lugar de prueba y las respuestas a corto plazo, pero si fueron encontradas asociaciones entre el lugar de prueba y las salidas a largo plazo. Los resultados obtenidos se explican a partir de la construcción de significado de la ciudad a partir del uso, por lo que, los significados de los entornos acústicos varían según el lugar de origen de los evaluadores y sus experiencias previas.

Como resultado de este proceso se presenta un modelo de evaluación de paisajes sonoros en laboratorio, un modelo de semiosis de paisajes sonoros, se aportan herramientas eficaces para el estudio de paisajes sonoros teniendo en cuenta parámetros binaurales y monoaurales y se discute la influencia de las variaciones de las fuentes sonoras en el plano horizontal en la percepción de atributos relacionados con la variabilidad del entorno acústico. Además, se realizan aportes en el estudio del contexto y su relación con la generación de significado de los entornos acústicos, información que puede ser utilizada para la gestión de los entornos acústicos urbanos, teniendo en cuenta no solo la influencia de los atributos perceptuales, sino también proyectando los posibles usos de los lugares. Así mismo se realizan aportes teóricos con conceptos como "no lugares sonoros" y "Oferta Sonora", útiles en los procesos de diseño de paisajes sonoros. 



\section{ABSTRACT}

This research discusses soundscape assessment from objective, subjective and descriptive aspects. For this, were analyzed thirteen urban soundscapes from Bogotá (Colombia), Brasilia (Brazil) and Lisbon (Portugal) cities.

The first part of this research focused on the search for acoustic parameters that allowed to describe alternative attributes to comfort and annoyance related to noise, considering spatial and temporal behavior of sound sources. At this stage, parameters derived from the functions of autocorrelation and interaural cross-correlation (ACF and IACF) in their running and long-term modalities were adapted and applied to the study of acoustic environments. Likewise, subjective evaluation tests and descriptive analysis were developed to have a wide evaluation of the environments and soundscape. High correlations were found between objective parameters and perceptual attributes, allowing to appreciate that variations of sound sources in the horizontal plane and temporal energy variations are associated with variable, agitated or monotonous environments. In addition, results obtained from statistical tests indicate significant differences between the acoustic environments according to acoustic parameters derived from the ACF and IACF and their keynote sound.

The second part of this research analyzed the influence of contextual and semiotics aspects in the assessment, design, and management of soundscapes, based on the comparison of in situ and laboratory evaluation methodologies and the study of the Person-Place Interaction component. Regarding the assessment methods comparison, in situ and laboratory tests were carried out in three urban parks of Lisbon, finding statistically significant differences according to the type of methodology used. In relation to the influence of Place-Person Interaction in the assessment of soundscapes, laboratory tests were developed in Bogota and Lisbon cities. In this experiment were presented audio fragments of acoustic environments from known and unknown places to the audiences from each city (showing to both groups the same places of Lisbon and Bogota). No considerable associations were found between the test site and short-term responses, but associations were found between the test site and the long-term outputs. The results obtained are explained by the construction of the meaning of the city upon the use, so that the meanings of the acoustic environments vary according to the evaluators' city of origin (and their previous experiences).

As a result of this process, the following are presented: 1) a laboratory soundscape assessment model and 2) a soundscape semiosis model. Likewise, effective tools are provided for the study of soundscapes considering binaural and monaural parameters. The influence of the variations of the sound sources in the horizontal plane on the perception of attributes related to the variability of the acoustic environment is discussed. In addition, contributions are made in the study of context and its relationship with the generation of the meaning of acoustic environments. This information can be used for the management of urban acoustic environments, considering not only the influence of perceptual attributes, but also projecting the possible uses of places. Likewise, theoretical contributions are made with concepts such as "sonorous non-places" and "Sound Affordance", useful in the design processes of soundscapes. 



\section{ÍNDICE GENERAL}

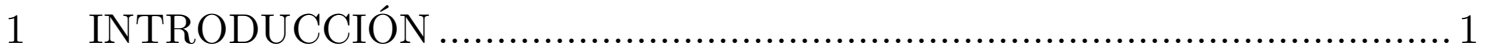

1.1 Ruido Urbano: Enfoque desde el control de ruido..................................... 1

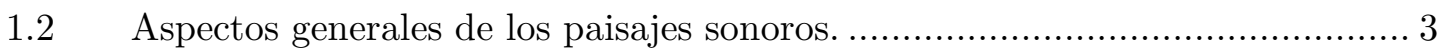

1.3 Estandarización del marco conceptual, recolección de datos y reportes de

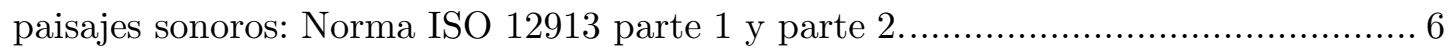

$1.4 \quad$ Paisaje sonoro y elementos contextuales..................................................11

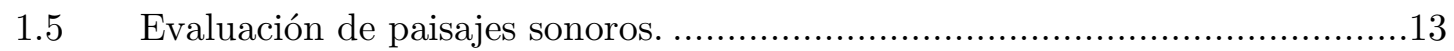

1.6 Funciones de autocorrelación y correlación cruzada interaural: aspectos

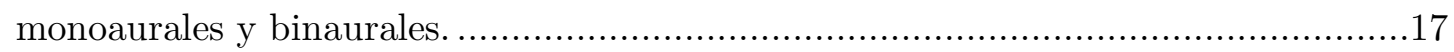

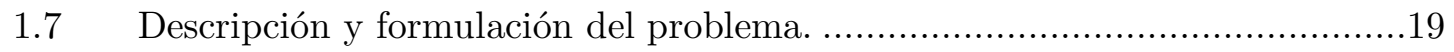

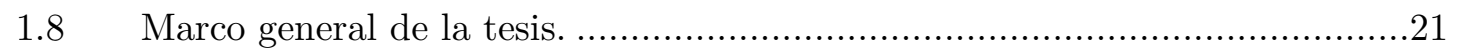

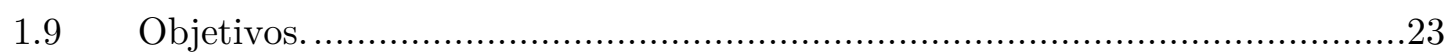

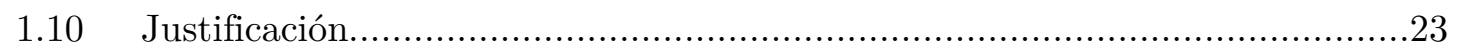

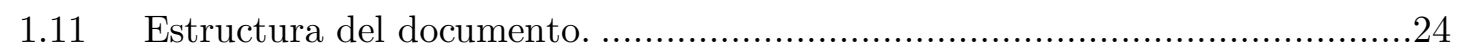

Referencias Primer Capítulo ....................................................... 26

2 SEGÚNDO CAPÍTULO. ANÁLISIS DE ASPECTOS ESPACIALES Y TEMPORALES EN LA EVALUACIÓN DE PAISAJES SONOROS: Adaptación e implementación de parámetros acústicos derivados de las funciones de autocorrelación (ACF) y correlación cruzada interaural (IACF) . ................... 31

2.1 Abordaje teórico de aspectos espaciales y temporales en la evaluación de paisajes sonoros.

2.1.1 Uso de parámetros acústicos en el estudio de paisajes y entornos acústicos. 32

2.1.2 Teoría de las ACF e IACF: Parámetros relacionados con comportamientos espaciales y temporales.

2.1.3 Evaluación subjetiva: Atributos perceptuales y modelos de evaluación

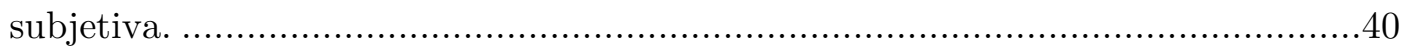

2.1.4 Aspectos descriptivos de entornos acústicos..........................................41

$2.2 \quad$ Abordaje metodológico y pruebas realizadas............................................42

2.2.1 Lugares en estudio y grabación de entornos acústicos.............................43

2.2.2 Evaluación objetiva: parámetros binaurales, temporales y energéticos. .....44

2.2.3 Aspectos descriptivos y evaluación subjetiva de los paisajes sonoros.........47

2.2.4 Procesado de datos y pruebas estadísticas. ...........................................50

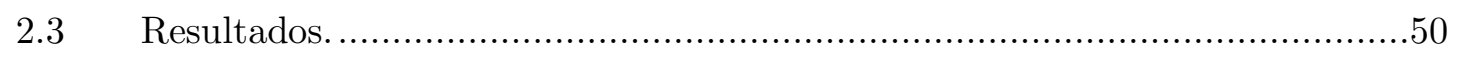

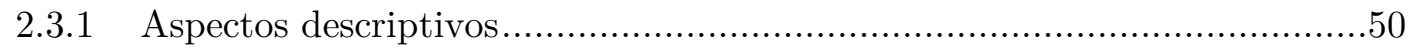

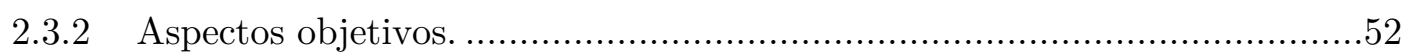

2.3.3 Aspectos subjetivos y estudios de correlación entre parámetros objetivos y

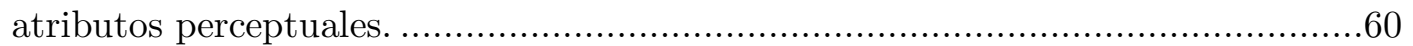

2.4 Discusión y conclusiones.......................................................................... 70 
3. PAISAJES SONOROS: RESPUESTAS Y SALIDAS Y SU RELACIÓN CON EL CONTEXTO.

3.1 Aproximación teórica: percepción ecológica, experiencia ambiental, calidad sonora y comunicación y semiótica en el estudio de paisajes sonoros urbanos. .........83

3.1.1 Psicología ecológica y enfoque tradicional de percepción...........................83

3.1.2 Modelo de experiencia ambiental aplicado a los paisajes sonoros. ..............85

3.1.3 Calidad sonora y elementos semióticos en el estudio de paisajes

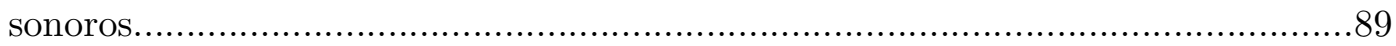

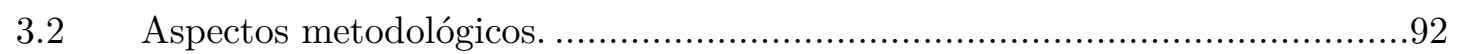

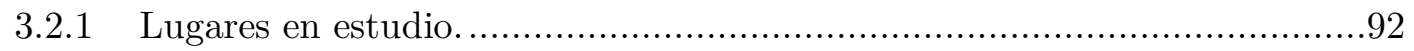

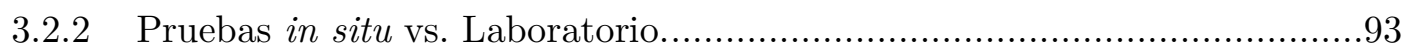

3.2.3 Pruebas según contexto del oyente: respuestas y salidas en evaluaciones de

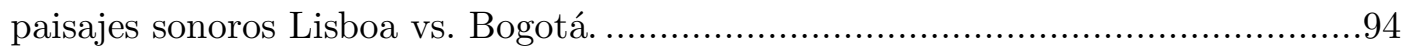

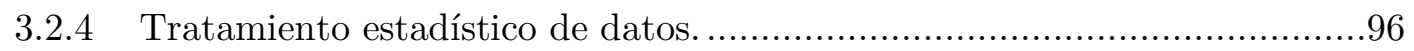

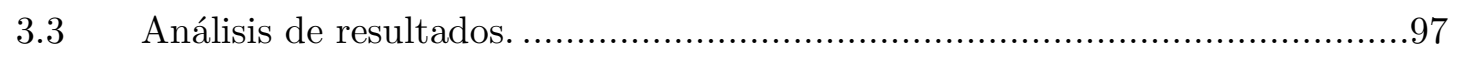

3.3.1 Comparación pruebas in situ vs. laboratorios. .....................................97

3.3.2 Evaluación en laboratorio de paisajes sonoros considerando el elemento interacción lugar-persona: comparación de evaluación de paisajes sonoros entre oyentes de las ciudades de Lisboa (Portugal) y Bogotá (Colombia)....................104

3.4 Discusión y conclusiones............................................................... 113

3.4.1 Discusión y conclusiones según comparación entre evaluaciones in situ vs. laboratorio.

3.4.2 Discusión y conclusiones de diferencias en respuestas y salidas según evaluadores de Lisboa y Bogotá.

3.4.3 Aspectos contextuales y semióticos aplicados a procesos de gestión, diseño y planeación de paisajes sonoros urbanos.

Referencias Tercer Capítulo...................................................................... 124

4 CONSIDERACIONES FINALES, CONCLUSIONES Y TRABAJOS A

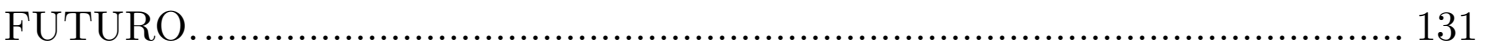

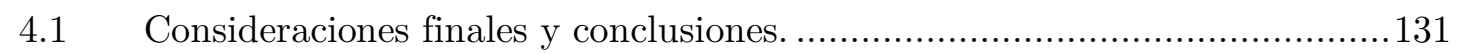

4.2 Líneas futuras de investigación.......................................................... 138

4 FINAL CONSIDERATIONS AND CONCLUSIONS. ........................... 143

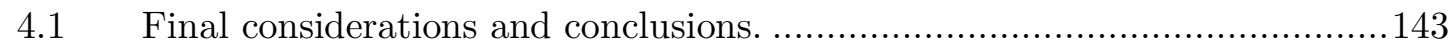

$4.2 \quad$ Future research lines. ........................................................................ 150 


\section{LISTADO DE ANEXOS}

Anexo 1. Presentación de lugares en estudio Bogotá y Brasil...

Anexo 2. Especificaciones técnicas del sistema de grabación binaural (datos

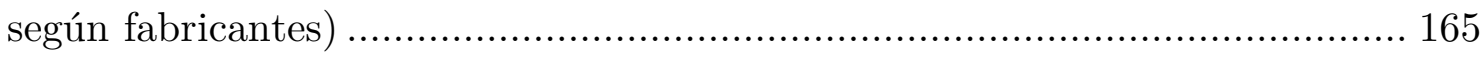

Anexo 3. Características y calibración del sistema de reproducción................ 169

Anexo 5. Lugares en estudio en Lisboa (Portugal)................................. 177

Anexo 6. Herramienta de evaluación in situ en Lisboa................................ 181

Anexo 7. Herramienta de evaluación en laboratorio en portugués. ................. 183

Anexo 8. Pruebas de normalidad de Shapiro-Wilk para los parques de Lisboa-

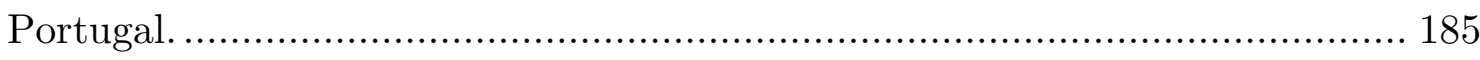





\section{LISTADO DE FIGURAS}

Figura 1-1. Porcentaje urbano y aglomeración urbana................................. 2

Figura 1-2. Pirámide de necesidades humanas de Maslow. .............................. 3

Figura 1-3. Modelo gráfico de paisaje sonoro presentado en Internoise 2010...... 5

Figura 1-4. Marco conceptual de paisajes sonoros según la norma ISO 12913-1..8

Figura 1-5. Modelo conceptual de experiencias ambientales que influencian la

construcción perceptual de los paisajes sonoros ................................... 12

Figura 1-6 Modelo bidimensional de respuesta emocional de paisajes sonoros propuesto por Axelsson, Nilsson y Gerglund.................................. 15

Figura 1-7 Modelo bidimensional de respuesta emocional de paisajes sonoros propuesto por Cain, Jennings y Poxon. ................................................... 16

Figura 1-8. Marco general de la teses doctoral............................................ 22

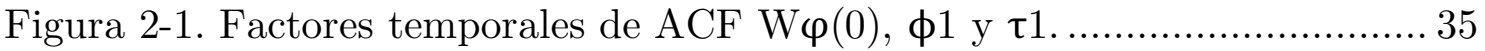

Figura 2-2. Duración efectiva de la envolvente normalizada de la ACF $\tau e . . . . . .36$

Figura 2-3. Función de autocorrelación móvil o de corta duración. .................. 37

Figura 2-4. Factores espaciales relacionados con el IACF........................... 40

Figura 2-5. Modelo Bidimensional de atributos perceptuales de Axelsson ........ 40

Figura 2-6. Clasificación de fuentes sonoras según modelo de Brown .............. 42

Figura 2-7. Diagrama de proceso de calibración para la grabación y reproducción de ambientes sonoros................................................. 49

Figura 2-8. Aspectos generales del proceso de evaluación subjetiva................. 50

Figura 2-9. Descriptores estadísticos para el parámetro IACC en señales con ponderación Z ....................................................................... 54

Figura 2-10. Descriptores estadísticos para el parámetro IACC en señales con

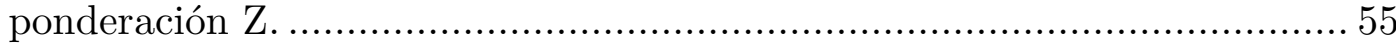

Figura 2-11. Descriptores estadísticos para el parámetro IACC en señales con ponderación Z ........................................................................... 56

Figura 2-12. Descriptores estadísticos para el parámetro $\tau$ IACC en señales con ponderación A

Figura 2-13. Descriptores estadísticos para el parámetro $\tau$ e en señales con ponderación Z.

Figura 2-14. Descriptores estadísticos para el parámetro te en señales con

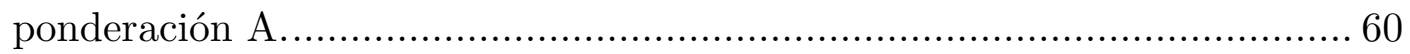

Figura 2-15. Representación gráfica de los lugares evaluados. ......................... 61

Figura 3-1. Modelo tradicional de percepción ........................................... 85

Figura 3-2. Proceso psicológico de experiencia ambiental ............................. 86

Figura 3-3. Modelo general conceptual de experiencia ambiental................... 87

Figura 3-4. Modelo de semiosis de Jekosch ............................................... 91 
Figura 3-5. Resumen pruebas de análisis de contexto.

Figura 3-6. Medias de atributos perceptuales (respuestas a corto plazo) en pruebas in situ y en laboratorio para parques de Lisboa-Portugal. 98

Figura 3-7. Medianas de atributos perceptuales (respuestas a corto plazo) en pruebas in situ y en laboratorio para parques de Lisboa-Portugal. 98

Figura 3-8 Representación gráfica de la evaluación de parques urbanos de la ciudad de Lisboa, usando como medida de tendencia central las medias. 102

Figura 3-9 Representación gráfica de la evaluación de parques urbanos de la ciudad de Lisboa, usando como medida de tendencia central las medianas.

Figura 3-10. Valores medios de atributos perceptuales Parte I (respuestas a corto plazo de lugares de Lisboa y Bogotá según el lugar de prueba) ...... 106

Figura 3-11.Valores medios de atributos perceptuales Parte II (respuestas a corto plazo de lugares de Lisboa y Bogotá según el lugar de evaluación) 106

Figura 3-12. Representación gráfica de la evaluación paisajes sonoros de las ciudades de Lisboa y Bogotá según evaluadores bogotanos y lisboetas usando como medida de tendencia central la media

Figura 3-13. Porcentajes de tiempos de permanencia de los evaluadores en lugares con entornos acústicos como los presentados en las grabaciones.. 110

Figura 3-14. Porcentajes de uso que darían los evaluadores en lugares con entornos acústicos como los presentados en las grabaciones.

Figura 3-15. Modelo de semiosis de paisajes sonoros urbanos 121

Figura 4-1. Modelo de evaluación subjetiva de paisajes sonoros en laboratorios.

Figure 4-1. Subjective assessment model of soundscapes in laboratories 148 


\section{LISTADO DE TABLAS}

Tabla 1-1. Resumen de atributos perceptuales según diferentes autores.... 16

Tabla 2-1. Parámetros acústicos objetivos usados para el análisis espacial y temporal de entornos acústicos.

Tabla 2-2. Descripción, listado de sonidos, sonidos fundamentales y categorías principal y secundaria a la que pertenecen los sonidos fundamentales ...... 51

Tabla 2-3. Valores de parámetros binaurales para los entornos acústicos con ponderación Z .............................................................................. 52

Tabla 2-4. Valores de parámetros binaurales para los entornos acústicos con ponderación A............................................................................. 53

Tabla 2-5. Resultados de las pruebas de Kruskal-Wallis con $\alpha=0.05$ para señales con ponderación $\mathrm{Z}$ según las categorías de sonidos fundamentales y parámetros binaurales

Tabla 2-6. Resultados de las pruebas de Kruskal-Wallis con $\alpha=0.05$ para señales con ponderación A según las categorías de sonidos fundamentales y parámetros binaurales 55

Tabla 2-7. Valores de parámetros temporales para los entornos acústicos con ponderación $\mathrm{Z}$

Tabla 2-8. Valores de parámetros temporales para los entornos acústicos con

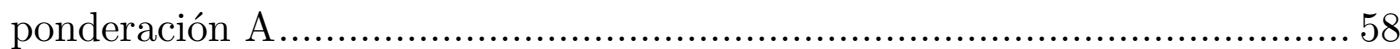

Tabla 2-9. Resultados de las pruebas de Kruskal-Wallis con $\alpha=0.05$ para señales con ponderación $\mathrm{Z}$ según las categorías de sonidos fundamentales y parámetros temporales.

Tabla 2-10. Resultados de las pruebas de Kruskal-Wallis con $\alpha=0.05$ para señales con ponderación A según las categorías de sonidos fundamentales y parámetros temporales

Tabla 2-11. Valores medios de parámetros subjetivos.

Tabla 2-12. Coeficientes de correlación de Spearman entre atributos perceptuales, parámetros binaurales y Leq con ponderación Z.

Tabla 2-13. Coeficientes de correlación de Spearman entre atributos perceptuales y parámetros binaurales con ponderación A.

Tabla 2-14. Coeficiente de correlación de Spearman entre atributos perceptuales y parámetros temporales con ponderación Z 68

Tabla 2-15. Coeficiente de correlación de Spearman entre atributos perceptuales y parámetros temporales con ponderación A.

Tabla 3-1. Desviaciones estándar de los tres parques en Lisboa-Portugal para las pruebas in situ vs. laboratorio 
Tabla 3-2. Resultados de las pruebas no paramétricas de Mann-Whitney para parques de Lisboa. Metodologías In situ. vs. Laboratorio

Tabla 3-3. Resultados de componentes principales según model de evaluación sueco.

Tabla 3-4. Resultados de pruebas de Mann-Whitney entre expertos y no expertos para parques de Lisboa (pruebas en laboratorio).

Tabla 3-5. Sonido Fundamental y nivel continuo equivalente ponderado A para los 8 paisajes sonoros en estudio.

Tabla 3-6. Resultados de las pruebas de U Mann-Whitney entre evaluadores de Bogotá y evaluadores de Lisboa. 107

Tabla 3-7. Resultado de las pruebas de Chi-cuadrado y Razón de verosimilitud para los 8 paisajes sonoros entre evaluadores de Bogotá y evaluadores de Lisboa

Tabla 3-8. Aspectos contextuales y semióticos aplicado a la guía de diseño de paisajes sonoros de Bento-Coelho 122

Tabla 7-A. Pruebas de normalidad de Shapiro-Wilk 185 
CAPÍTULO 1

\section{INTRODUCCIÓN}




\section{INTRODUCCIÓN}

\subsection{Ruido Urbano: Enfoque desde el control de ruido.}

Según datos del Departamento de asuntos económicos y sociales de Naciones Unidas, el 55\% de la población mundial vive en centros urbanos [1]. En Europa la cifra se incrementa a un $74 \%$, mientras que en América Latina y países del Caribe es del 81\% (Figura 1-1). Teniendo en cuenta que el mismo informe prevé que para el año 2050 estas cifras se incrementarán al $68.4 \%$ a nivel mundial (en Europa y América latina llegarán al $83.7 \%$ y $87.8 \%$ respectivamente), los problemas y desafíos de los entornos urbanos están lejos de desaparecer y la gestión acústica ambiental no se escapa de ello.

Tráfico rodado, aéreo y ferroviario, actividades comerciales, así como prácticas culturales son algunos de los responsables de emisiones sonoras en los ambientes urbanos, los cuales a altos niveles pueden generar un impacto directo en la salud y en la calidad de vida de los habitantes de la ciudad [2-6]. Estas emisiones se convierten en un peligro potencial que puede afectar la salud de un gran porcentaje de la población mundial, por lo que la Organización Mundial de la Salud ha venido generando documentos en donde, además de advertir de los riesgos a los que se exponen los habitantes de las ciudades (perturbación del sueño, hipertensión arterial, efectos fisiológicos y en la salud mental), se presentan recomendaciones, guías y criterios para la medición, control y disminución de dichas emisiones, así como para la preservación de las denominadas zonas tranquilas [7-11].

Atendiendo a la necesidad de generar herramientas que regulen las emisiones acústicas, distintos países y regiones han creado directivas y legislaciones que evalúan y controlan los niveles de ruido ambiental. Para los países miembros de la Unión Europea las directivas 2002/49/CE y 2015/996 establecen enfoques comunes para evitar, prevenir o reducir efectos nocivos relacionados con la exposición al ruido ambiental, así como los métodos comunes en la evaluación de ruido [12,13]. Estas directivas tienen como objetivo la generación de diagnosis acústicas mediante mapas estratégicos de ruido (útil en la definición de políticas y estrategias de planeamiento urbano), planes de acción 
(a fin de corregir y mejorar el ambiente urbano enfocado al control de ruido) y la difusión de la información (tanto a la ciudadanía como a la comisión europea). A partir de la directiva 2002/49/CE, los diferentes países miembros de la Unión Europea establecen los máximos valores de ruido permitidos según el uso del suelo, definiendo además zonas sensibles al ruido. Este modelo de política acústica ambiental, con un enfoque predominante en el control de los niveles de presión sonora, ha sido usado en otros países del mundo debido las fortalezas y ventajas que ofrece respecto al planeamiento urbanístico [14].

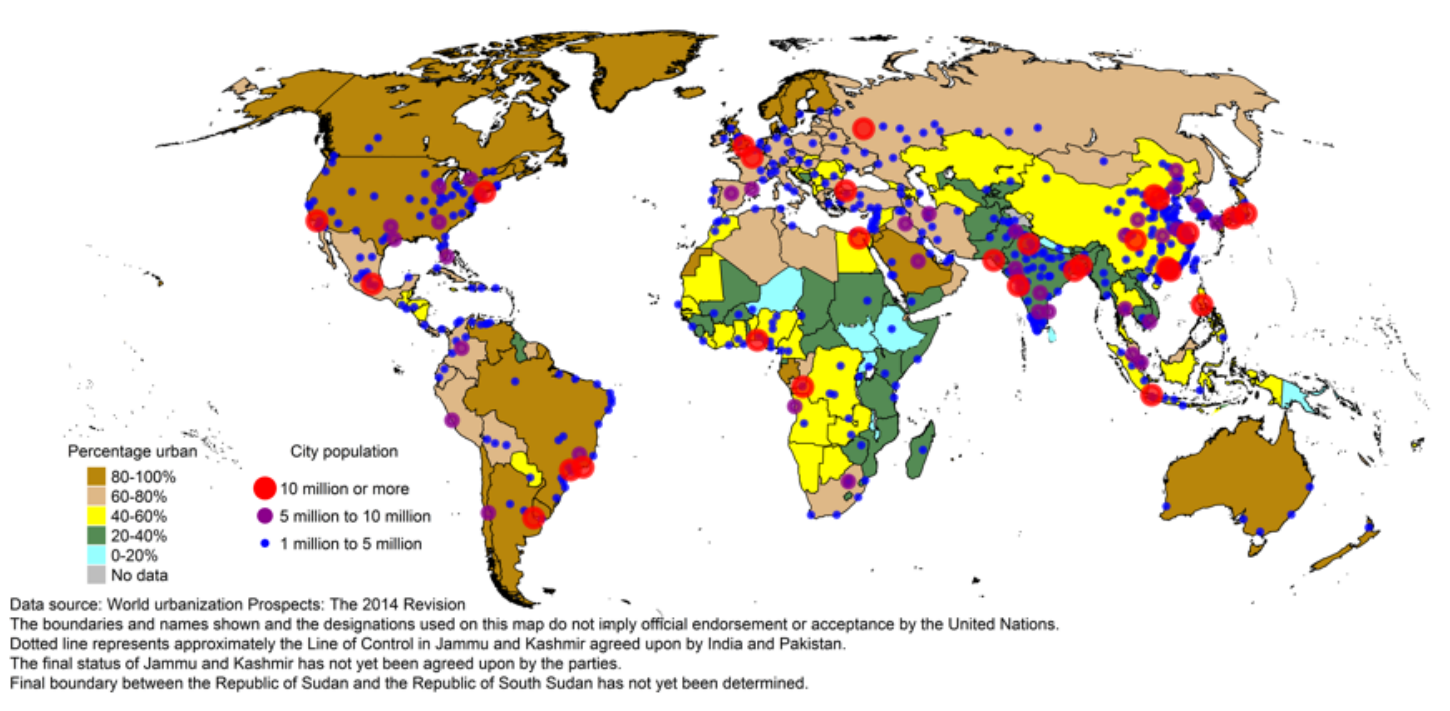

Figura 1-1. Porcentaje urbano y aglomeración urbana. (Fuente: Departamento de asuntos económicos y sociales de Naciones Unida, 2018)

Partiendo de que toda emisión sonora que pueda generar un impacto en la salud debe ser controlada, los modelos de gestión acústica ambiental no deben reducirse únicamente al control de los niveles de ruido. En muchas ocasiones los niveles sonoros pueden superar los máximos niveles de ruido permitidos y aun así no ser considerados molestos. Estas situaciones han sido descritas en diversos trabajos, donde lugares con altos niveles de sonoros no necesariamente eran considerados molestos, así como aquellos con bajos niveles sonoros no siempre eran los más confortables $[15,16]$.

Así mismo, a medida que las sociedades evolucionan, sus necesidades también lo hacen, siendo este el principio de la pirámide de Maslow (Figura 1-2). Según Maslow existen cinco niveles de necesidades, las cuales se agrupan a su vez en tres niveles: necesidades básicas, necesidades psicológicas y necesidades de autocumplimiento. Dentro del primer grupo, necesidades básicas, se encuentran 
las necesidades fisiológicas (necesidades como comer, dormir, etc.) y las necesidades de seguridad (leyes, seguridad física, orden, estabilidad). En el segundo grupo, de necesidades psicológicas, se encuentran las necesidades de afiliación (amor, afectos, amistad) y reconocimiento (confianza, respeto, éxito). En el tercer grupo se encuentra la necesidad de autorrealización (creatividad, espontaneidad y desarrollo total de potencialidades) [17]. Si bien no es objeto de esta tesis entrar a discutir la validez de la propuesta de Maslow, sí resulta interesante traer este modelo al campo de la acústica ambiental y el planeamiento urbano. Esto debido a que, aunque el enfoque de control de ruido permite cumplir con aspectos fisiológicos de la pirámide de Maslow (evitar el impacto del ruido en la salud), necesidades como calidad de vida y disfrute del entorno (que podrían estar encuadradas en el segundo nivel de necesidades) no son atendidas.

Necesidades

de autorrealización

Necesidades de reconocimiento

Necesidades de afiliación

Necesidades de seguridad

Necesidades fisiológicas

Figura 1-2. Pirámide de necesidades humanas de Maslow.

En este sentido, el modelo convencional compuesto por fuente sonora, entorno acústico y evaluación de molestia o impacto en la salud usado en normas y legislaciones relacionadas con ruido ambiental está siendo fuertemente discutido [18]. Se hace necesario que las normas y legislaciones, además de aspectos fisiológicos y de molestia frente al ruido, tengan en cuenta aspectos perceptivos, culturales, sociológicos y semióticos, propendiendo de esta forma por mejorar la calidad de vida de las personas. En otras palabras, empieza a ser necesario un enfoque desde el concepto de paisajes sonoros.

\subsection{Aspectos generales de los paisajes sonoros.}

Según Murray Schafer, considerado como uno de los precursores de este concepto, el término de paisaje sonoro se refiere al ambiente sonoro de un lugar 
determinado [19]. Derivado del término inglés landscape, el concepto de soundscape ha venido desarrollándose de formas diversas, aunque actualmente todos coinciden en que el marco general es la percepción que tiene el ser humano del ambiente sonoro, esto según su contexto [2]. Para Traux, los entornos sonoros deben ser estudiados como un sistema de comunicación, concentrándose en la relación entre el individuo y el entorno [20,21]. Esta mirada, enmarcada en el campo de la ecología acústica, requiere de un trabajo multidisciplinar, donde además de caracterizar y describir el entorno acústico (aspectos abordados generalmente desde campos ingenieriles), se deben tener en cuenta aspectos como significado del sonido para los pobladores (semiótica y sociología) y percepción que las personas tienen de los entornos acústicos según el contexto (psicología) [22-24].

Desde el punto de vista conceptual, Schafer propone inicialmente tres grandes clasificaciones de sonidos que componen los paisajes sonoros: sonidos fundamentales, marcas sonoras y señales sonoras. Los sonidos fundamentales son aquellos que son escuchados continuamente o que se presentan de forma constante, de tal forma que estos crean un fondo contra el cual los otros sonidos son percibidos. Las marcas sonoras derivan del término landmark y se refieren a los sonidos que son característicos de una comunidad, los cuales pueden ser únicos o que poseen cualidades que los hacen especialmente notados. Si se relacionan los sonidos fundamentales con las marcas sonoras se puede decir, en términos de imágenes visuales, que los sonidos fundamentales son el fondo de la imagen y las marcas sonoras son la forma [19]. Las señales sonoras son aquellos sonidos a los cuales la atención es particularmente direccionada. Estos conceptos son presentados en esta tesis debido a que, como ha sido expresado en otros trabajos, son útiles en la descripción y comprensión de paisajes sonoros [25].

Sin embargo, debido a las diferentes vertientes ecológicas, ingenieriles y hasta artísticas, en las que ha sido usado este concepto, no ha sido fácil el consenso de un método para el estudio y evaluación de paisajes sonoros. Ambiente acústico, ambiente sónico, ambiente sonoro, espacio aural y el ambiente de sonido son solo algunos de los conceptos por los que ha pasado el término de paisaje sonoro en años recientes. Solo hasta el año 2013, con la publicación de la ISO 12913-1. Acoustics - Soundscape. Part 1. Definition and conceptual framework, es posible 
comenzar la estandarización de aspectos conceptuales. A continuación se analizan algunos de los trabajos más relevantes que contribuyeron con este proceso de estandarización.

En el año 2010 se celebró en Lisboa (Portugal) el International Congress on Noise Control Engineering (Internoise), enfocado en ruido y sostenibilidad. En este evento fue discutido el modelo de descripción gráfico del concepto de paisaje sonoro [18]. La figura 1-3 se presenta el modelo propuesto. Este está compuesto por cinco cajas menores y contrasta fuertemente con el proceso de evaluación clásica de ruido comunitario (que presenta tres etapas: fuentes sonoras, entorno acústico del área receptora y grado de molestia). A diferencia del ya mencionado modelo de control de ruido tradicional, en este modelo destaca principalmente la aparición de los componentes contexto y percepción. Así mismo, se tienen en cuenta las reacciones (outcoments) del ser humano, así como los caminos de flujo de información.

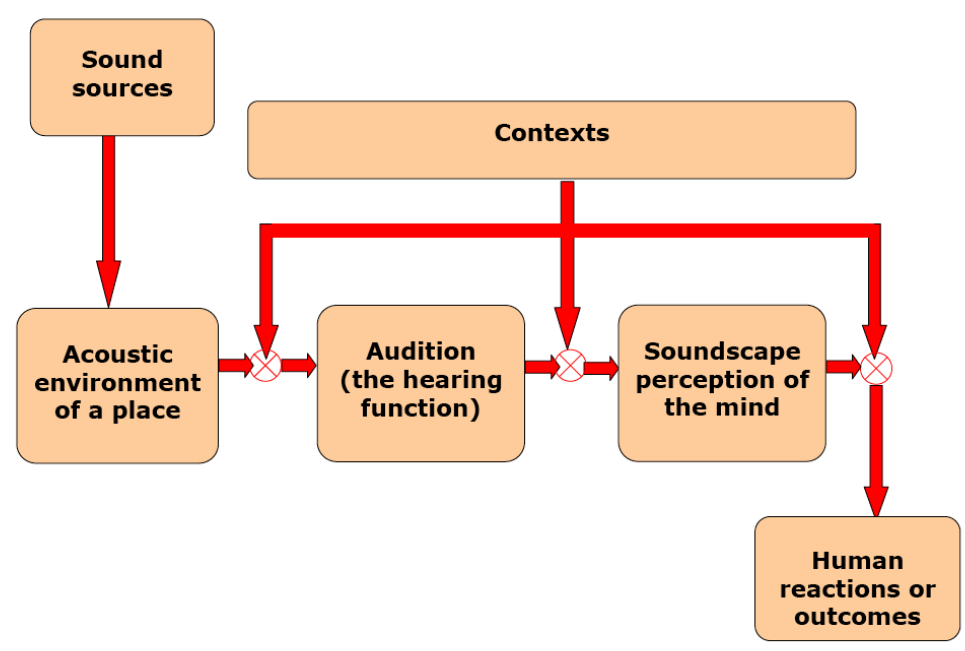

Figura 1-3. Modelo gráfico de paisaje sonoro presentado en Internoise 2010, Lisboa (Portugal) [18].

El modelo presentado en la figura 1-3 implica que la respuesta del ser humano no genera una reacción simple por parte del ser humano (molestia y/o confort), puesto que puede tener múltiples "salidas" o respuestas. Además, esas salidas no son una función directa de la fuente sonora y el ambiente acústico, sino de la percepción del ser humano. Así mismo, la percepción y respuesta del ser humano está en función del contexto en que el entorno acústico es percibido.

Considerando que en el enfoque de paisaje sonoro el ser humano es quien experimenta el entorno acústico, otro aspecto importante en el desarrollo de este 
concepto está relacionado con la estandarización de metodologías, cuestionarios, identificación de sonidos y evaluación de la calidad del paisaje sonoro. Es por ello que los procesos de evaluación de paisajes sonoros requieren de un riguroso estudio del contexto (incluyendo aspectos visuales y de configuración arquitectónica), de una buena clasificación de las fuentes sonoras (lo que debe incluir una taxonomía sonora), de una caracterización detallada de los entornos acústicos (a partir de descriptores energéticos, temporales, binaurales y de parámetros psicoacústicos) así como de una detallada evaluación subjetiva que indique actitudes y comportamientos de las personas. Para lo anterior se requiere de cuestionarios y procesos de evaluación subjetiva, así como métodos de procesamiento de datos y presentación de resultados claramente establecidos $[2,26]$.

Por otra parte, se ha buscado consolidar el concepto de paisajes sonoros como herramienta que permita no solo hacer control de ruido en espacios públicos, sino también pensar en el diseño, gestión y/o administración de ciudad considerando aspectos sonoros [16,25,27-31]. En este sentido, algunos trabajos han propuesto modelos donde la composición del paisaje sonoro en términos objetivos, y la inclusión de factores pueden afectar una percepción positiva del ambiente [27].

En la actualidad la norma ISO 12913 en sus partes 1 y 2 presentan un marco conceptual de paisaje sonoro, así como requerimientos para la recolección de datos y reportes. A continuación se presentan los aspectos más relevantes relacionados con estas normas.

\subsection{Estandarización del marco conceptual, recolección de datos y reportes de paisajes sonoros: Norma ISO 12913 parte 1 y parte 2.}

En el año 2013 la International Organization for Standarization ISO publica la primera sección de la norma relacionada con los paisajes sonoros: la ISO 12913-1, Acoustics - Soundscape. Part 1. Definition and conceptual framework. Esta norma provee definiciones y el marco conceptual para la descripción de paisajes sonoros, explicando los factores relevantes para la medición y descripción de paisajes sonoros, así como para su planificación, diseño y gestión. Además de incluir la definición de paisaje sonoro y ambiente acústico, se presenta 
un marco conceptual de paisajes sonoros, el cual es presentado en la figura 1-4 $[32]$.

La norma ISO 12913-1 define los paisajes sonoros como la percepción, experimentación y/o comprensión del entorno acústico mediada por el contexto, siendo el entorno acústico el sonido de todas las fuentes sonoras que fueron modificadas por el ambiente. Estos entornos acústicos pueden ser reales o simulados [32]. De esta forma se puede apreciar una clara diferencia entre paisaje sonoro y entorno acústico, diferencia que también ha sido remarcada trabajos previos [2,33]. Un concepto abarca el otro y sus análisis deben ofrecer información clara pero diferenciada. En relación al estudio de entornos sonoros, este se ha enfocado en dos frentes: el primero relacionado con la descripción y clasificación de los entornos acústicos y de las fuentes sonoras que los componen [18,26,34-36], y el segundo centrado en el estudio del comportamiento energético del campo sonoro, usando para ello descriptores de promediación energética temporal (así como otros descriptores estadísticos como percentiles y máximos y mínimos) y en algunos casos descriptores psicoacústicos [37-41].

Respecto a la percepción del entorno acústico, concepto global de paisaje sonoro, han sido propuestos diversos modelos de evaluación subjetiva que introdujeron atributos perceptuales como actividad, placer y cantidad de eventos por nombrar algunos ejemplos, así como también se han desarrollado trabajos que analizaron la influencia de otros estímulos (principalmente visuales) en la percepción del entorno acústico [15,21,42-52]. Las metodologías de evaluación subjetiva pueden dividirse en dos: pruebas in situ y pruebas en laboratorio. La sección 1.5 profundiza en los métodos de evaluación de paisajes sonoros.

Volviendo al modelo conceptual de paisajes sonoros presentado en la Figura 1-4, este presenta siete aspectos principales: 1. Contexto, 2. Fuentes sonoras, 3. Entorno acústico, 4. Sensación auditiva, 5. Interpretación de la sensación auditiva, 6. Respuestas y 7. Salidas y se busca que la medición, juicio o evaluación del paisaje sonoro se haga mediante la percepción humana del paisaje sonoro (Figura 1-4). 


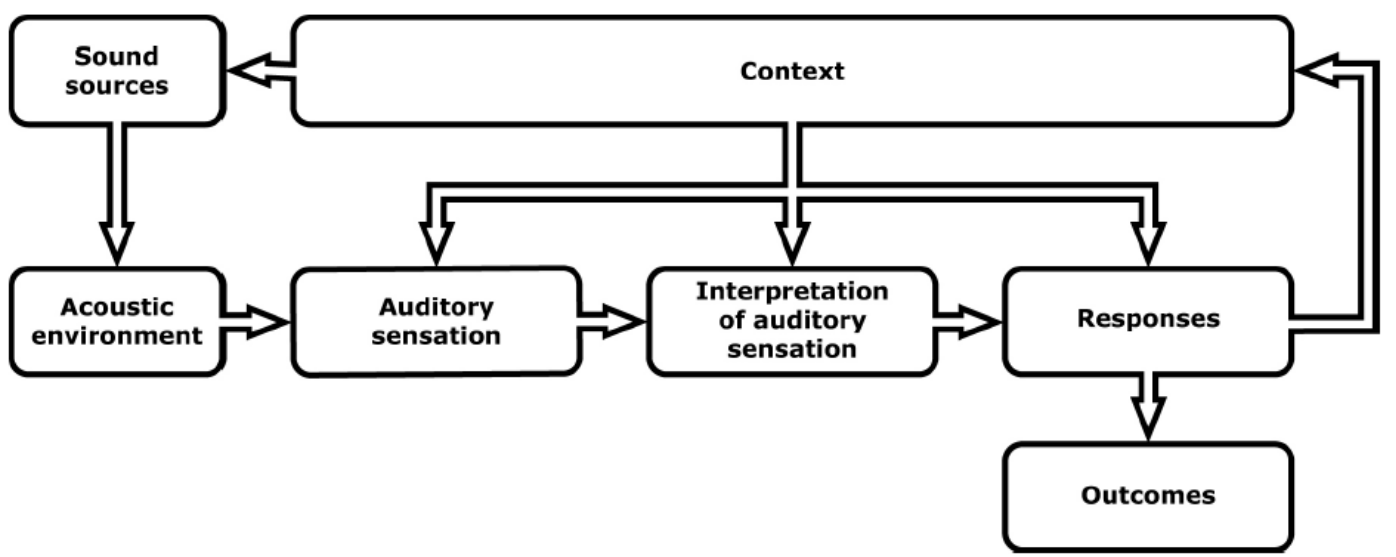

Figura 1-4. Marco conceptual de paisajes sonoros según la norma ISO 12913-1. Acoustics Soundscape. Part 1. Definition and conceptual framework.

Como se puede apreciar, se trata de un modelo mucho más completo que el presentado en la figura 1-3, incluyendo la interrelación entre persona-actividadespacio, así como la relación espacio-tiempo. El contexto puede influenciar la sensación auditiva, la interpretación de la sensación auditiva y las respuestas del ambiente acústico. Así mismo la norma propone que la fuente sonora es el origen de los paisajes sonoros por lo que debe hacerse un buen análisis de su distribución tanto en el espacio como en el tiempo.

Según esta norma, el ambiente o entorno acústico hace referencia a los sonidos de todas las fuentes sonoras que fueron modificadas por el ambiente. Esta modificación incluye condiciones de propagación, reflexión, difracción, condiciones meteorológicas, absorción y reverberación.

En relación a la sensación auditiva, para la norma ISO 12913-1, esta es una función neurológica que inicia cuando los estímulos auditivos alcanzan los receptores del sistema auditivo. Esta es la primera etapa del proceso de detección y representación de un ambiente o entorno acústico. Los caminos de los oídos al cerebro están separados y el sonido (creado en la corteza auditiva) se compone de una mezcla de las dos señales neuronales [32]. Por lo anterior, la escucha binaural está influenciada por el enmascaramiento, el contenido espectral y temporal, así como la distribución espacial de las fuentes sonoras. En este sentido, a pesar de que el concepto de paisaje sonoro busca evaluar la percepción que el ser humano tiene del entorno acústico, llama la atención cómo los análisis objetivos usan principalmente parámetros acústicos de carácter monoaural, ignorando 
información relacionada con la espacialidad, ubicación y movimiento de las fuentes sonoras existente en el entorno acústico y el impacto en la percepción de estos. Si bien diferentes estudios recomiendan el uso de grabaciones binaurales para el estudio de los entornos acústicos [53,54], son pocos los trabajos que exploran el uso de descriptores binaurales aplicados con estos propósitos [36,55$57]$.

Para la interpretación de la sensación auditiva, la norma ISO 12913-1 hace referencia al procesado, consciente o inconsciente, de crear información valiosa que puede llevar a la comprensión o entendimiento del entorno acústico. La comprensión de dicho entorno representa una experiencia del entorno acústico.

Pasando a las respuestas y salidas, las primeras se relacionan con reacciones a corto plazo y emociones, así como el comportamiento, que puede cambiar según el contexto, mientras que las segundas son una consecuencia general a largo plazo e incluyen actitudes (ej. creencias, juicios y hábitos). Puede apreciarse en el modelo propuesto que el contexto influencia prácticamente todos los componentes que lo conforman, aunque no se especifica la forma como lo hace.

Pasando a la norma ISO 12913-2 Acoustics - Soundscape. Part 2. Data collection and reporting requirements, del año 2018, esta presenta aspectos de recolección y reporte de datos para el estudio de paisajes sonoros considerando tres elementos fundamentales: las personas, el entorno acústico y el contexto. En referencia a las personas, la norma indica la necesidad de describir ampliamente el tipo de participante de las pruebas de evaluación de paisajes sonoros (si son residentes, o visitantes), la forma en que dichos participantes fueron seleccionados, su conocimiento y experiencia en el campo del conocimiento, edad y género entre otros aspectos. En relación al entorno acústico, la norma especifica que se debe detallar si el entorno acústico es real o simulado, siendo necesario describir aspectos taxonómicos de las fuentes sonoras (el anexo C presenta la aplicación del modelo de Brown) así como recomendando la combinación de indicadores de ruido y parámetros psicoacústicos para la caracterización de dicho entorno. En referencia a los aspectos contextuales, este documento solo indica que la información contextual debe ser reportada en detalle, aunque no especifica sobre cuales aspectos se debe detallar. 
Así mismo, desde aspectos conceptuales, las norma ISO 12913-2 establece una diferencia entre descriptores e indicadores, donde el primer término se refiere a la descripción de la percepción de cualquier entorno acústico (es decir que es accionado por la persona), mientras que el segundo es un término relaciona con la predicción de un descriptor (o de una parte de este) [58]. En otras palabras, la norma presenta la necesidad de fortalecer la conexión entre aspectos objetivos y subjetivos, dependiendo del tipo de estudio y/o evaluación que se esté realizando.

Finalmente, la norma especifica las formas de obtención de datos de aspectos objetivos, a partir de caminatas sonoras, cuestionarios y entrevistas, recomendando que los procesos de medición de entornos acústicos sean realizados con sistemas binaurales. Los anexos B y C presentan ejemplos de cuestionarios e indicadores potencialmente útiles en el estudio de paisajes sonoros, mientras que el anexo D detalla aspectos de posicionamiento y aspectos técnicos de la grabación binaural.

Se considera que las normas 12913 parte 1 y parte 2 son un buen inicio para la estandarización del estudio de paisajes sonoros. La definición general de paisajes sonoros y su marco conceptual la parte 1 de la norma es clara, mientras que la parte 2 de la norma presenta tres elementos claves sobre los cuales deben desarrollarse el estudio de paisajes sonoros. Sin embargo, vale la pena analizar lo siguiente:

- A pesar de presentar un marco conceptual claro y completo, la norma 12913-1 plantea muchas más preguntas que respuestas, principalmente relacionadas con procesos y métodos necesarios para poder estudiar y gestionar paisajes sonoros.

- Si bien la 12913-2 clarifica aspectos relacionados con la obtención de datos del entorno acústico (principalmente respecto a aspectos taxonómicos), la recomendación acerca del uso de "indicadores de ruido" en estudio de paisajes sonoros, así como la carencia de indicadores binaurales siguen en contravía de caracterizar entornos acústicos de la forma más cercana a la cual el ser humano los percibe.

- Tanto la norma 12913 en sus dos partes, como los estudios presentados en esta sección sugieren la necesidad de evaluar paisajes sonoros a partir de grabaciones y sistemas binaurales. Sin embargo, en el campo de la 
acústica ambiental, estos temas han sido poco desarrollados. Lo anterior deja preguntas como ¿qué tipos de atributos binaurales pueden ser usados en el estudio de paisajes sonoros? Esta interrogante expone uno de los aspectos más importantes a tratar en esta tesis doctoral.

- La descripción del contexto, elemento clave en todas las discusiones anteriormente citadas, no es clara en cuanto a los elementos que pueden ser tenidos en cuenta. Quedan preguntas relacionadas con ¿cómo determinar la afectación del contexto en los paisajes sonoros? ¿cómo puede influir el contexto en aspectos relacionados con la gestión y diseño de paisajes sonoros?

- Es interesante la distinción entre respuestas y salidas que toma el oyente. La primera se relaciona con reacciones a corto plazo generadas por el paisaje sonoro y la segunda relacionada con decisiones a largo plazo. Sin embargo, los trabajos referenciados y la norma ISO 12913-2 se centran en la obtención de datos relacionados con las respuestas a corto plazo, por lo que datos referentes a las salidas no son considerados. Además, sigue sin esclarecerse la forma en que dichas respuestas y salidas se ven afectadas por el contexto.

- Para la descripción del lugar y el estudio del contexto se hace necesario incluir aspectos visuales, estéticos y de significado entre otros, aunque si no se especifica con claridad los elementos a tener en cuenta en este ítem, las informaciones pueden ser difusas.

Por lo anterior, la norma ISO 12913 es una invitación a futuros desarrollos metodológicos de evaluación y gestión de paisajes sonoros, ofreciendo un marco conceptual claro y una propuesta de recolección y presentación de datos. Sin embargo, procesos de evaluación y elementos contextuales deben seguirse profundizando para estudios efectivos de paisajes sonoros. A continuación se presentan algunos trabajos relevantes relacionados con elementos contextuales y procesos de evaluación de paisajes sonoros.

\subsection{Paisaje sonoro y elementos contextuales.}

La Figura 1-4 presenta un marco conceptual donde sus diferentes componentes se ven afectados de forma directa o indirecta por el contexto. Sin 
embargo, dicho marco plantea de nuevo muchos interrogantes relacionadas con los aspectos que lo componen y la forma en que puede ser evaluada. En este aspecto, en el año 2010 se proponen un modelo conceptual de experiencias ambientales que influencian la construcción perceptual de los paisajes sonoros [59] (figura 1-5). El modelo está compuesto por tres elementos principales: la persona, el lugar y actividad, los cuales deben ser tenidos en cuenta en el estudio o valoración de los paisajes sonoros [33,48,59-61].

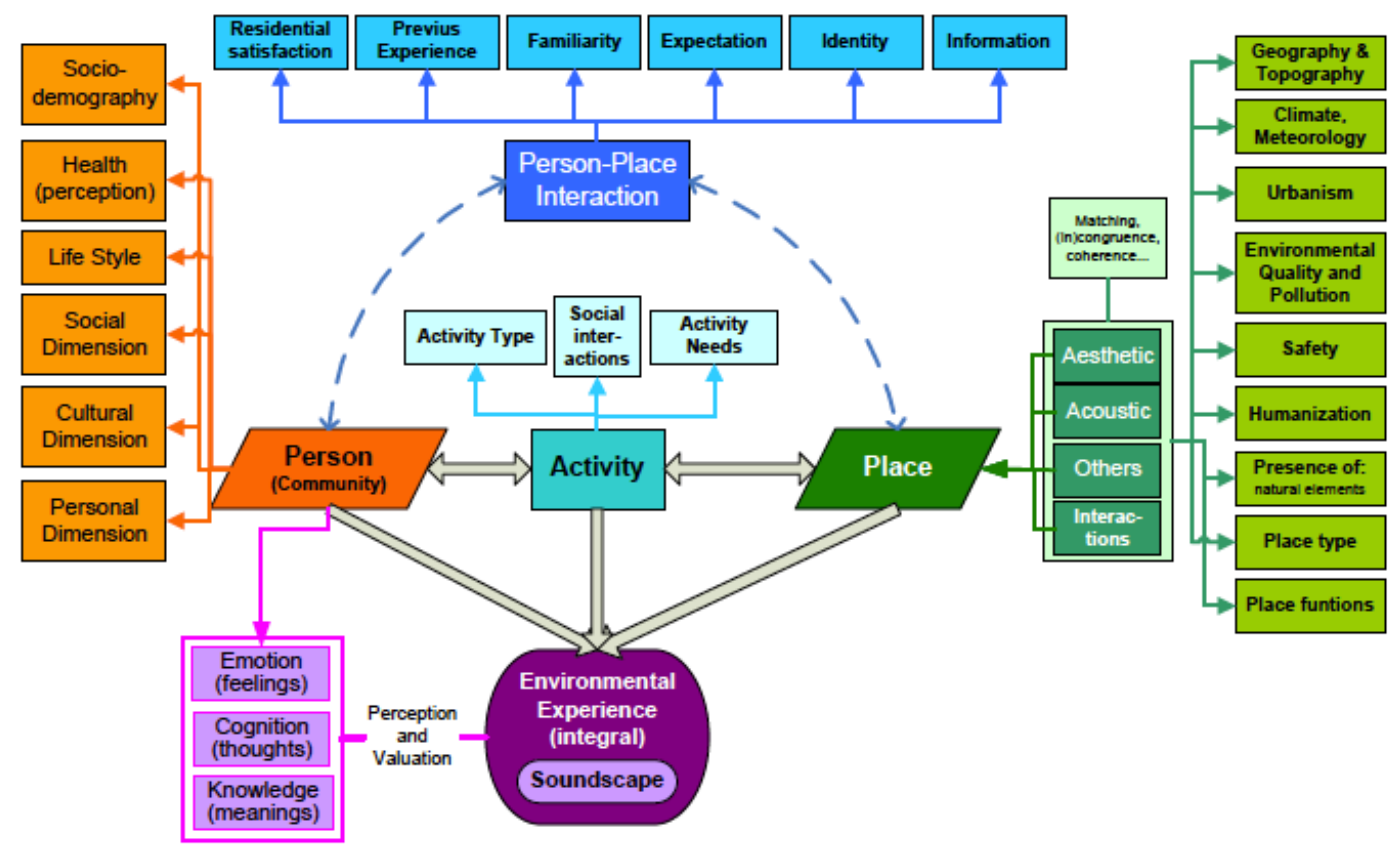

Figura 1-5. Modelo conceptual de experiencias ambientales que influencian la construcción perceptual de los paisajes sonoros (Herranz-Pascual et al, 2010)

El modelo presentado en la figura 1-5 permite apreciar una gran cantidad de aspectos que influencian la percepción de paisajes sonoros, aunque no ofrece herramientas para su análisis y evaluación, así como tampoco establece el grado de influencia de las diferentes variables en las respuestas y salidas. Aunque existen trabajos que analizan la relación contexto-percepción, la mayoría hacen énfasis en la importancia de los estímulos visuales en la percepción de los paisajes sonoros. En esta sección se presentan los aportes más importantes relacionados con la relación estímulos visuales y estímulos auditivos.

Respecto a la influencia de estímulos visuales en la percepción paisajes sonoros, varios trabajos demuestran que los paisajes sonoros urbanos pueden ser caracterizados por las marcas sonoras (soundmarks), mientras que la percepción general del paisaje sonoro es dominada por el confort acústico, las imágenes 
visuales y la luz del día [45]. También se han desarrollado métodos que permiten evaluar la tranquilidad de los espacios rurales y urbanos respecto a estímulos visuales y acústicos, donde los descriptores visuales y auditivos claves que influencian la tranquilidad dentro de los paisajes sonoros son el nivel de presión sonora máximo ponderado A (LAmax) y los porcentajes de características naturales presentes en los paisajes visuales (NF) [46]. En la misma línea se pueden encontrar trabajos que analizan la influencia de características físicas de paisajes visuales en la percepción de paisajes sonoros en diferentes tipos de espacios $[15,47]$.

Así mismo, considerando el elemento interacción lugar-persona, presentado en el modelo de Herranz-Pascual, han sido desarrollados trabajos relacionados con la comparación de la percepción de paisajes sonoros en parques de diferentes parques del mundo, la comparación de la percepción de paisajes sonoros entre turistas y residentes, así como la comparación de la evaluación de paisajes sonoros entre expertos y no expertos en el campo de conocimiento acústico [24,62-65].

Los trabajos presentados en esta sección permiten apreciar la importancia del contexto en los paisajes sonoros. Sin embargo, dentro del modelo de HerranzPascual et. al presentado en la figura 1-5 todavía quedan demasiadas interrogantes que resolver, como por ejemplo el grado de influencia entre las experiencias previas de los oyentes en los lugares y la percepción y significado de paisajes sonoros, así como la relación entre contexto-percepción. Para abordar esta problemática, se hace necesario primero determinar diferentes aspectos relacionados con la evaluación de paisajes sonoros.

\subsection{Evaluación de paisajes sonoros.}

En esta sección se hará énfasis en los métodos de evaluación in situ y de laboratorio, modelos de evaluación perceptual, así como trabajos relacionados con parámetros binaurales.

Las pruebas in situ pueden desarrollarse a partir de caminatas sonoras (sound walks) o puntos de escucha fijos, donde los evaluadores presentan su opinión respecto a diferentes atributos del entorno acústico en el que se encuentran [66]. Se recomienda que las pruebas subjetivas se desarrollen a partir de cuestionarios, o entrevistas guiadas [58]. Generalmente son usadas pruebas de 
tipo diferencial semántico o de escala de respuestas, aunque también es común realizar entrevistas y preguntas abiertas a fin de no limitar la posibilidad de respuestas de los evaluadores. Al ser una prueba in situ, el entorno ofrece estímulos visuales, olfativos y táctiles, lo que permite una contextualización total a los evaluadores respecto a su entorno, por lo que los resultados obtenidos reflejan no solo aspectos sonoros sino también la complejidad del mundo real [15,67-71]

Pasando a las pruebas en laboratorio, estas buscan tener un mayor control de las diferentes variables a las que está expuesto el evaluador, de tal forma que, en lo posible, a este le sean presentadas únicamente los estímulos de interés para la investigación. Además de las pruebas de tipo diferencial semántico o escala de respuestas, es posible aplicar pruebas como pares comparados con las que se pueden obtener escalas de Thurstone o de Likert [38,46,51,52,72-75]. Uno de las principales ventajas de este tipo de pruebas es que permite presentar al oyente entornos sonoros simulados, aspecto clave en el diseño de paisajes sonoros.

Respecto a los modelos perceptuales, después de una visita realizada por Schafer a Estocolmo en 1998, se crearon dos programas que a futuro permitieron generar dos modelos bidimensionales que describen la respuesta emocional basado en los paisajes sonoros. El primer programa denominado "Soundscape support to health", fue desarrollado entre 1999 y el 2007 fue liderado por Berglund (en Suecia), mientras que el segundo programa fue desarrollado entre el 2006 y 2009 por un equipo interdisciplinar en Reino Unido denominado Positive Soundscape Project [76]. Como principales resultados de estos dos proyectos se obtuvo la descripción de los paisajes sonoros mediante el modelo bidimensional de respuesta emocional, el cual fue descrito mediante la relación Eventfulness-Pleasantness [51], o Calmness-Vibrant [52]. A continuación se profundiza en cada uno de ellos.

Iniciando por el trabajo de Axelsson, Nilsson y Berglund, los resultados de este trabajo se asemejan a los obtenidos en emociones y el entorno psicológico del modelo circumplejo afectivo de Russell [77], donde se identifican dos dimensiones relacionadas con la percepción placentera de los ambientes (figura 1-6). Este trabajo sugiere que las propiedades informativas de los paisajes sonoros (ej. categorías de sonidos) son mejores descriptores de percepción de la calidad de los paisajes sonoros que los descriptores de ruido usados en mediciones acústicas (ej. Leq). Finalmente, los investigadores derivan en un modelo empírico que permite 
integrar el gran número de atributos perceptuales potenciales en un pequeño número de dimensiones básicas de percepción de paisajes sonoros. Así mismo, dentro del trabajo se determinó la relación entre esas dimensiones básicas perceptuales y las propiedades físicas de los paisajes sonoros (lo que incluye sonidos dominantes y variables acústicas).

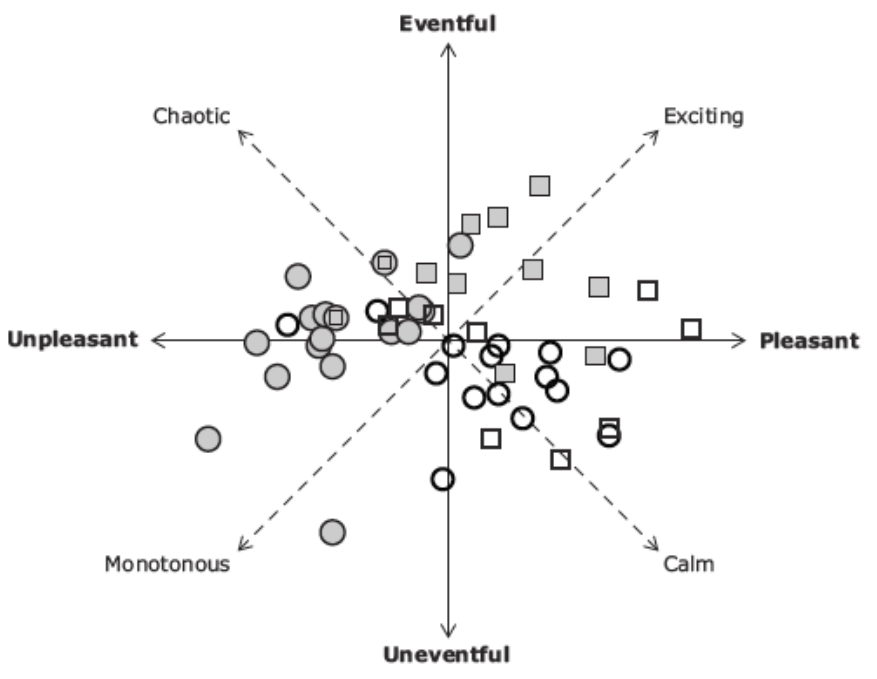

Figura 1-6 Modelo bidimensional de respuesta emocional de paisajes sonoros propuesto por Axelsson, Nilsson y Gerglund

Pasando al modelo desarrollado a partir de proyecto Positive Soundscape, este también presenta un modelo bidimensional, pero en este caso obtenido por medio del uso de la técnica de diferencial semántico (figura 1-7). Esta técnica es frecuentemente usada en la valoración de eventos auditivos y permite identificar dimensiones psicológicas a lo largo de los cuales se evalúan diferentes estímulos sensoriales y funciona por la clasificación de una serie de entidades (como paisajes sonoros), en una serie de escalas bipolares tales como calmo-perturbado. En este trabajo se realizaron grandes esfuerzos en analizar cómo el lugar hace sentir a las personas, pues sería una información útil para los planificadores y diseñadores urbanos, por lo que, según los autores no se trata solo describir el sonido, sino describir lo que ese sonido hace sentir al ser escuchado.

Si bien estos dos modelos de atributos perceptuales son los más recientes, el aporte realizado por muchos otros investigadores ha sido fundamental en el proceso de ampliación de atributos perceptuales alternativos al confort y molestia frente al ruido $[42,43,45,46,78]$. La tabla $1-1$ presenta un resumen de dichos atributos perceptuales. 


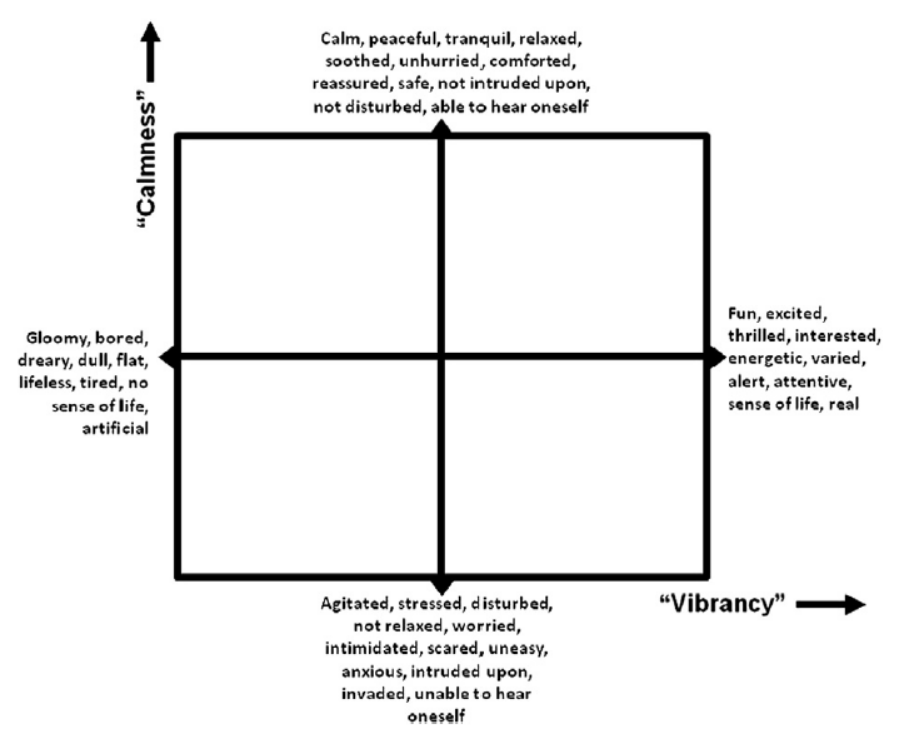

Figura 1-7 Modelo bidimensional de respuesta emocional de paisajes sonoros propuesto por Cain, Jennings y Poxon.

Tabla 1-1. Resumen de atributos perceptuales según diferentes autores. En verde atributos asociados al placer y confort, en azul atributos asociados a la actividad y diversidad de lugar. Elaboración propia.

\begin{tabular}{|c|c|c|}
\hline General Dimensions & Perceptual Attributes & Author \\
\hline Calmness and Relaxation & \multirow{2}{*}{$\begin{array}{l}\text { Calm, peaceful, tranquil, relaxed, comforted, safe. } \\
\text { Fun, excited, thrilled, energetic, varied, alert, sense } \\
\text { of life, real. }\end{array}$} & \multirow[b]{2}{*}{ Cain et al. (2013) [52] } \\
\hline Vibrancy and Arousal & & \\
\hline $\begin{array}{l}\text { Emotional evaluation and } \\
\text { strength }\end{array}$ & \multirow{2}{*}{$\begin{array}{l}\text { Comfortable, pleasant, relaxing, nice, liberating, } \\
\text { silent, weak, human, interesting, safe. } \\
\text { Animated, informative, varied, } \\
\text { clear. }\end{array}$} & \multirow{2}{*}{$\begin{array}{l}\text { Guillén and López } \\
\text { Barrio (2007) [42] }\end{array}$} \\
\hline $\begin{array}{l}\text { Activity } \\
\text { Clarity }\end{array}$ & & \\
\hline Pleasantness & Comfortable, appealing, inviting. & \multirow{2}{*}{$\begin{array}{l}\text { Axelsson et al. (2010) } \\
{[51]}\end{array}$} \\
\hline Eventfulness & Eventful, full of life, mobile. & \\
\hline Preference & & \multirow{2}{*}{$\begin{array}{l}\text { Kawai et al. (2004) } \\
{[43]}\end{array}$} \\
\hline $\begin{array}{l}\text { Activity } \\
\text { Sense of life }\end{array}$ & & \\
\hline $\begin{array}{l}\text { Comfort, loudness, and } \\
\text { pitch sensations }\end{array}$ & $\begin{array}{l}\text { Comfort, quiet, harmonious, soft, weak, light, } \\
\text { pleasant, warm }\end{array}$ & \multirow[t]{2}{*}{ Jeon et al. (2010) [78] } \\
\hline Temporal variation & Monotonous, unique & \\
\hline
\end{tabular}

La tabla 1-1 permite apreciar que entre los diferentes modelos existen aspectos comunes, pudiéndose identificar dos grupos. Un primer grupo presenta dimensiones generales como Pleasantness y Comfort, las cuales aparecen en los modelos de Axelsson, Cain y Jeon. El segundo grupo presenta como elementos comunes aspectos relacionados con Activity, Vibrancy, Eventfulness y Temporal 
variation, los cuales aparecen en todos los modelos. En relación al primer grupo, diversos estudios presentan correlaciones aceptables entre estos atributos perceptuales y parámetros acústicos convencionales como nivel continuo equivalente ponderado A $[51,78]$, lo cual concuerda con el enfoque tradicional de acústica relacionado con que altos niveles de ruido generan molestia. Sin embargo, el segundo grupo de atributos es mucho más complejo de describir desde parámetros acústicos convencionales, dado que indican actividad, movimiento, cantidad de eventos y variación temporal. Descriptores energéticos como el LeqA así como diferentes estadísticos descriptivos como Máximos, Mínimos, Percentiles 10 y 90 no presentan grandes correlaciones con el segundo grupo de atributos perceptuales. Para entender este fenómeno es importante establecer que esa actividad y movimiento puede derivarse de variaciones temporales y espaciales (en el plano horizontal) de las diferentes fuentes sonoras que conforman el paisaje sonoro. Por lo anterior, es lógico que parámetros que se refieren a comportamientos energéticos monoaurales (promedios, mínimos, máximos, etc.) no presenten correlaciones con atributos que precisan de una descripción binaural y temporal. En ese sentido se considera necesario abandonar el campo de la acústica ambiental y explorar otro tipo de parámetros acústicos que puedan ser útiles en propósitos.

\subsection{Funciones de autocorrelación y correlación cruzada interaural: aspectos monoaurales y binaurales.}

Parámetros acústicos derivados de las funciones de autocorrelación y de las funciones de correlación cruzada interaural han sido ampliamente empleadas para la descripción de comportamientos espaciales y temporales en el campo de la acústica arquitectónica [79-81]. Según Yoichi Ando, la función de autocorrelación (ACF) describe las señales monoaurales de cada uno de los dos oídos y es usada frecuentemente para evaluar comportamientos temporales y la percepción de cualidades tonales, permitiendo determinar tono, timbre, sonoridad y duración. La función de correlación cruzada interaural (IACF) describe la correlación entre las dos señales monuarales y es usada para evaluar los atributos espaciales, permitiendo el análisis de sensaciones espaciales como la localización en el plano 
horizontal, el ancho aparente de la fuente (ASW) y el envolvimiento subjetivo $[80]$.

Si bien es cierto que la teoría de Ando ha sido aplicada principalmente al estudio de campos sonoros y salas de concierto, es interesante ver como la teoría de preferencias subjetivas se ha aplicado también a las molestias de ruido. A continuación, se presentan algunos trabajos relevantes.

En relación a la aplicación de $\mathrm{ACF}$ en el estudio de molestia de ruido respecto a factores temporales, se han desarrollado pruebas donde se grabaron ruidos tonales (motos) y ruidos no tonales, se calcularon diferentes factores de ACF, parámetros acústicos tradicionales (SPL, L50, Leq, etc) y se aplicaron pruebas subjetivas [82]. Dentro de los resultados de este trabajo no se encontró correlación con el SPL, aunque si se encontró buena correlación con otros indicadores estimados como Var_SPL (Varianza del SPL que representa fluctuaciones en sonoridad), tiempo efectivo de duración te (que representan la variación en la fuerza del tono y su coherencia temporal), y Var_c1 (que representa las fluctuaciones en el tono y en el timbre). Se encontró mayor molestia en los ruidos tonales que en los no tonales, esto por los resultados de la correlación y los comentarios de los sujetos. Como resultado del trabajo se propuso una ecuación lineal que permite estimar la escala de molestia de forma aproximada, teniendo una correlación de $\mathrm{p}=0,91$.

Respecto a la molestia de ruido teniendo en cuenta factores espaciales, dos experimentos evalúan los efectos de los factores espaciales extraídos del IACF para las molestias de ruido usando estímulos en laboratorio [83]. En el primer caso se usaron pruebas por pares comparativos para que las personas evaluaran la molestia generada por las fluctuaciones en la correlación cruzada interaural IACC y en el nivel de presión sonora SPL. En el segundo caso analizaron la molestia (de nuevo usando pruebas por pares comparados) en los cambios del retraso temporal interaural $\tau_{\mathrm{IACC}}$, y el SPL. Se encontró que la molestia se incrementaba con el incremento de IACC y las fluctuaciones de $\tau_{\mathrm{IACC}}$. Así mismo proponen dos expresiones para la descripción de la molestia frente al ruido, una en términos de Flu_IACC y SPL (con un coeficiente de correlación de 0,99) y la segunda en términos de Flu_t $\tau_{\text {IACC }}$ y el SPL (con un coeficiente de correlación de 0,989 . 
Como puede apreciarse en esta sección, desde hace varias décadas se vienen aplicando parámetros acústicos binaurales y monoaurales en estudios en el campo de acústica arquitectónica y la molestia frente al ruido. Se considera que, si bien la caracterización energética de los entornos acústicos es importante, este solo es uno de los aspectos que deben ser abordados en el estudio de paisajes sonoros. La inclusión de parámetros que permitan profundizar en aspectos temporales y espaciales puede ser potencialmente útil en la descripción de entornos acústicos y en el estudio de la correlación entre aspectos objetivos y subjetivos, por lo que serán temas importantes para tratar en el desarrollo de esta tesis doctoral.

\subsection{Descripción y formulación del problema.}

Tomando en consideración lo expuesto en los apartados anteriores, a continuación se presentan algunos comentarios que permiten llegar a la descripción del problema de esta tesis.

a. La gestión ambiental de entornos urbanos no puede reducirse exclusivamente a la percepción de molestia de ruido y la caracterización física a partir de descriptores energéticos acústicos. Si bien este debe ser el primer paso, evitando que los altos niveles de emisiones sonoras impacten en la salud de las personas, es necesario diseñar, planear y gestionar entornos que permitan alcanzar una mejor calidad de vida.

b. En lo que respecta a la norma ISO 12913 partes 1 y 2 y el estudio de contexto presentado en este capítulo, han sido encontrados avances en aspectos claves relacionados con el estudio de la percepción de entornos acústicos tales como la influencia del contexto en la percepción sonora, la discriminación entre respuestas a corto plazo (percepciones) y salidas a largo plazo (decisiones) y aspectos básicos relacionados con recolección y presentación de datos. Sin embargo, aun deben esclarecerse aspectos relacionados la evaluación de objetivos y subjetivos y su relación con aspectos contextuales.

c. En lo referente a aspectos contextuales, y considerando la importancia de este elemento en la evaluación de paisajes sonoros, si bien han sido desarrollados trabajos relacionados con el impacto de estímulos visuales en los paisajes sonoros, se hace necesario indagar en otros aspectos 
contextuales, como el grado de influencia entre las experiencias previas de los oyentes en los lugares y la percepción de paisajes sonoros, así como la relación entre contexto-percepción y la interacción lugarpersona.

d. Los modelos de percepción de paisajes sonoros, así como los diferentes atributos perceptuales presentados por diversos autores, permiten ampliar el espectro de atributos en entornos acústicos (normalmente relacionados con confort y molestia frente al ruido). La inclusión de atributos como actividad, cantidad de eventos y vitalidad, por citar unos pocos ejemplos, implican también la necesidad de explorar parámetros acústicos que permitan describir y correlacionar aspectos subjetivos con aspectos objetivos. La evaluación objetiva relacionada exclusivamente con parámetros energéticos no permite apreciar variaciones temporales ni espaciales de las fuentes sonoras que conforman los entornos acústicos.

e. Así mismo, a pesar de buscar cómo evaluar la percepción del ser humano del ambiente sonoro, son pocos los estudios que hacen uso y/o proponen descriptores binaurales acústicos en el estudio de entornos acústicos. El uso exclusivo de parámetros monoaurales en la evaluación de paisajes sonoros es de por si un contra sentido, dado que el concepto de paisajes sonoros involucra directamente procesos perceptuales binaurales.

De esta forma, esta tesis se desarrolla a partir de dos preguntas principales, que a su vez involucran otra serie de interrogantes. Las preguntas a las que se desea dar respuesta con esta tesis son:

¿Cómo evaluar paisajes sonoros, de forma que se obtenga información temporal y espacial que permita una conexión entre aspectos objetivos y subjetivos? Esta pregunta involucra interrogantes como ¿Cuál es la influencia de la variación de las fuentes sonoras en el plano horizontal respecto a atributos perceptuales relacionados con la variabilidad de los paisajes sonoros? ¿Esta influencia puede reflejarse también en variaciones temporales energéticas? ¿Qué parámetros acústicos permiten la descripción de dichos comportamientos? 
La segunda pregunta principal se relaciona con ¿Cuál es la influencia del contexto en la evaluación de los paisajes sonoros según el conocimiento y experiencia previa de los oyentes en los lugares y el tipo de metodología usada en el proceso de evaluación? De esta pregunta se desprenden preguntas como ¿Existen diferencias estadísticamente significativas en las respuestas de los oyentes dependiendo del tipo de metodología usada en la evaluación (in situ vs. laboratorio)? ¿A qué aspectos específicos de las respuestas afecta? ¿Existen diferencias estadísticamente significativas en las respuestas y salidas en grupos de oyentes con y sin experiencia y/o conocimiento del entorno evaluado? ¿Varia el significado del lugar según el contexto?

\subsection{Marco general de la tesis.}

Teniendo en cuenta la sección 1.7, esta tesis doctoral se enfoca en cuatro aspectos de los paisajes sonoros según se presenta en la figura 1-8: 1) Análisis objetivo, 2) Análisis Subjetivo, 3) Análisis descriptivo y 4) Influencia del contexto.

El análisis objetivo se fundamenta en la caracterización de entornos acústicos, considerando aspectos energéticos, espaciales y temporales. Para ello se usaron parámetros acústicos asociados con las ACF e IACF así como descriptores energéticos tradicionales.

En el análisis subjetivo se tuvieron en cuenta respuestas (mediante evaluaciones in situ y en laboratorio aplicando el modelo de Axelsson que incluye ocho atributos perceptuales, la evaluación general del entorno acústico y la estimación de los componentes principales Pleassantness y Eventfulness) y salidas (teniendo en cuenta tiempos de permanencia y posibles usos). Los análisis objetivos y subjetivos se conectan a partir de los estudios de correlación entre parámetros objetivos y las respuestas de los oyentes (atributos perceptuales). 


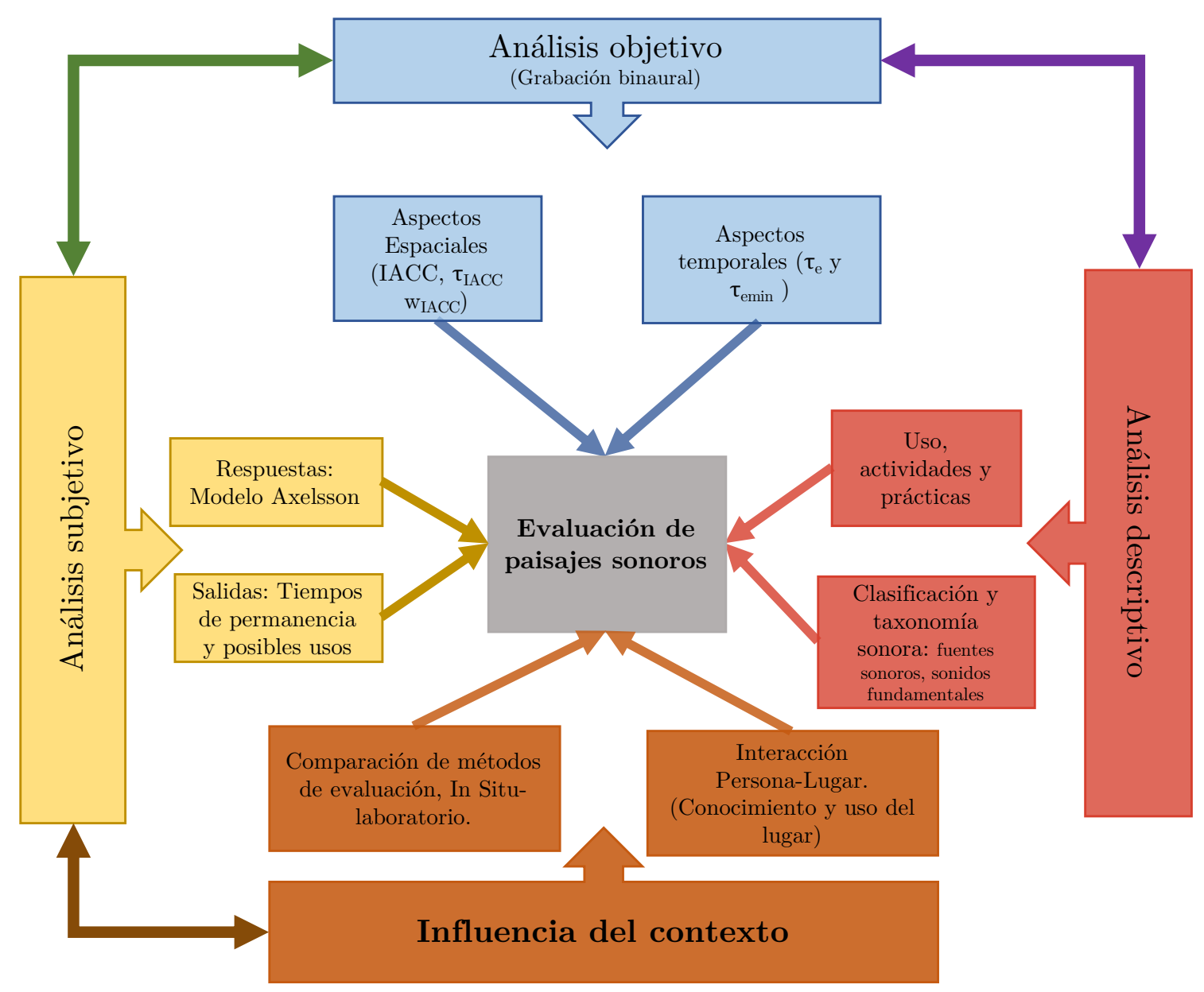

Figura 1-8. Marco general de la teses doctoral.

Para el análisis descriptivo se tuvieron en cuenta usos, actividades y prácticas, así como elementos de clasificación de fuentes sonoras y sonidos fundamentales de los paisajes sonoros. Los análisis objetivos y descriptivos se conectan a partir de análisis estadísticos que buscan establecer diferencias estadísticamente significativas entre los entornos acústicos en estudio según su parámetro acústico y su sonido fundamental.

Por último, se estudia la influencia del contexto en la evaluación de paisajes sonoros, esto a partir de la comparación de metodologías de evaluación in situ y en laboratorio y del estudio del componente interacción lugar-persona. Los puntos de conexión entre el análisis subjetivo y la influencia del contexto se generan a partir del estudio de las variaciones en las respuestas de los oyentes según el tipo de metodología usada (in situ o laboratorio) y en el estudio de la influencia del 
conocimiento del lugar y origen de los oyentes en las respuestas y salidas de los paisajes sonoros.

\subsection{Objetivos.}

Los objetivos propuestos para esta tesis son:

\section{General:}

Desarrollar un modelo de evaluación de paisajes sonoros urbanos según aspectos espaciales, temporales, subjetivos y de contexto.

\section{Objetivos específicos:}

- Establecer parámetros acústicos asociados a las funciones de autocorrelación y correlación cruzada interaural que permitan el análisis de aspectos temporales y espaciales de entornos acústicos urbanos.

- Determinar la aplicabilidad de parámetros acústicos asociados a las funciones de autocorrelación y correlación cruzada interaural en el análisis de paisajes sonoros a partir de estudios de correlación entre aspectos objetivos y subjetivos.

- Analizar la influencia en las salidas y respuestas de paisajes sonoros por parte de la interacción lugar-persona (según conocimiento de lugares) y de tipos de metodologías de evaluación.

\subsection{Justificación}

Como ha sido expuesto en este documento, existe una tendencia en el campo de la acústica ambiental que procura determinar la percepción que tiene el oyente de un espacio sonoro, teniendo en cuenta el contexto. Esa tendencia implica conlleva al desafío de desarrollar modelos de evaluación de paisajes sonoros que permitan establecer una conexión entre los parámetros objetivos, parámetros subjetivos y elementos contextuales. La evaluación de paisajes sonoros 
considerando esta triada, así como de la necesidad de aportar datos potencialmente útiles en procesos de diseño, planeación y gestión de paisajes sonoros son las razones por las que esta tesis doctoral se construye y se justifica.

Respecto a la evaluación objetiva, esta tesis doctoral se justifica considerando el vacío encontrado en el estado del arte respecto al uso de parámetros acústicos que se relacionen de forma efectiva con la gran diversidad de atributos perceptuales propuestos bajo el enfoque de paisajes sonoros.

Así mismo, el elemento contextual es clave en el estudio de paisajes sonoros. Sin embargo, los estudios de relación entre aspectos contextuales y paisajes sonoros se enfocan principalmente en la influencia de estímulos alternativos a los sonoros (principalmente visuales), dejando de lado otros aspectos contextuales como la interacción lugar-persona.

Por lo anterior, esta tesis encuentra justificación en la necesidad de profundizar en el estudio de paisajes sonoros mediante un abordaje objetivo, subjetivo y contextual que ofrezca una mirada acorde al enfoque multidisciplinar propuesto bajo el concepto de paisajes sonoros. Esto sin olvidar la necesidad latente de trabajar en la estandarización de métodos de evaluación que clarifiquen las diferentes dudas y preguntas que deja la norma ISO 12913-1.

\subsection{Estructura del documento.}

Este capítulo presentó una introducción al concepto de paisajes sonoros, una descripción detallada de la problemática abordada, los objetivos de la tesis, así como el marco general de la investigación. A continuación se describen brevemente el contenido y la estructura de los diferentes capítulos que el lector encontrará en este documento.

El segundo capítulo presenta la aplicación de parámetros acústicos asociados con las ACF e IACF en el estudio de paisajes sonoros. Para ello se realiza un abordaje teórico de las $\mathrm{ACF}$ e IACF en estudios de comportamientos temporales y espaciales, se explica el modelo de evaluación subjetiva de Östen Axelsson y el modelo de clasificación de fuentes sonoras de Lex Brown, ambos usados en esta tesis. Se presentan las metodologías usadas en los estudios de evaluación objetiva y subjetiva, así como los resultados obtenidos de las pruebas 
de correlación entre estos dos enfoques. Los experimentos desarrollados incluyen el estudio de diez entornos acústicos de ciudades de Bogotá (Colombia) y Brasilia (Brasil) (los cuales fueron analizados mediante diferentes parámetros acústicos asociados a las ACF e IACF), la aplicación de pruebas subjetivas en laboratorio (pruebas desarrolladas en la ciudad de Bogotá), los estudios de correlación entre aspectos subjetivos y objetivos, así como los estudios de detección de diferencias estadísticamente significativa entre los entornos acústicos según su parámetro acústico y su sonido fundamental. Finalmente se cierra con la discusión y conclusiones referentes a este capítulo.

El tercer capítulo se enfoca en aspectos contextuales relacionados con la influencia de la relación Persona-Lugar con las respuestas y salidas del oyente, así como la comparación entre metodologías de evaluación in situ y en laboratorio y su impacto en la respuesta de los oyentes. Se realiza un abordaje teórico desde conceptos como calidad sonora, relación lugar-significado y modelo de contexto aplicado a paisajes sonoros. Los experimentos presentados en este capítulo son dos: comparación entre metodologías de evaluación in situ y laboratorio aplicado a estudios de parques urbanos en la ciudad de Lisboa, y comparación entre respuestas y salidas de oyentes según relación Persona-Lugar (comparación de evaluación de paisajes sonoros en las ciudades de Lisboa (Portugal) y Bogotá (Colombia)). Se presenta la discusión y las conclusiones relacionada con los aspectos abordados en este capítulo.

El cuarto y último capítulo presenta las consideraciones finales de la tesis doctoral, indicando las conclusiones generales, así como las recomendaciones finales, posibles líneas de investigación y trabajos a futuro. Por último se presenta un modelo de evaluación de paisajes sonoros en laboratorio. 


\section{Referencias Primer Capítulo}

[1] United Nations, Department of Economic and Social Affairs PD. World Urbanization Prospects: The 2018 Revision, Online Edition. 2018. doi:10.1016/j.ijgo.2007.03.023.

[2] Brown AL, Kang J, Gjestland T. Towards standardization in soundscape preference assessment. Appl Acoust 2011;72:387-92. doi:10.1016/j.apacoust.2011.01.001.

[3] Van Kamp I, Klaeboe R, Brown AL, Lercher P. Soundscapes, Human Restoration and Quality of life. In: Kang J, Schulte-Fortkamp B, editors. Soundscape Built Environ., CRC Press; 2016, p. 43-68.

[4] Lercher P, Van Kamp I, Lindern E Von, Botteldooren D. Perceived Soundscapes and Health-Related Quality of Life, Context, Restoration, and Personal Characteristics. In: Kang J, Schulte-Fortkamp B, editors. Soundscape Built Environ., CRC Press; 2016, p. 133-60.

[5] Clark C, Paunovic K. Who environmental noise guidelines for the European region: A systematic review on environmental noise and quality of life, wellbeing and mental health. Int J Environ Res Public Health 2018;15. doi:10.3390/ijerph15112400.

[6] Medvedev O, Shepherd D, Hautus MJ. The restorative potential of soundscapes: A physiological investigation. Appl Acoust 2015;96. doi:10.1016/j.apacoust.2015.03.004.

[7] Berglund B, Lindvall T, Schwela D. Guidelines for community noise. 1999. doi:10.1260/0957456001497535.

[8] World Health Organization. Burden of disease from environmental noise. vol. 23. Copenhagen: 2011.

[9] World Health Organization. Night noise guidelines for Europe. 2009. doi:10.1093/ejechocard/jer095.

[10] World Health Organization (WHO). Environmental Noise guidelines for the European Region. Copenhagen: World Health Organization Regional Office for Europe; 2018. doi:10.3390/ijerph14080873.

[11] European Environmental Agency. Good practice guide on quiet areas. Luxembourg: European Environmental Agency; 2014. doi:10.2800/12611.

[12] Comision Europea. Directiva 2002/49/CE del Parlamento Europeo y del Consejo de 25 de junio de 2002 sobre evaluación y gestión del ruido ambiental. Minist La Pres España 2002:25.

[13] Comision Europea. Directiva (UE) 2015/996 de la comisión de 19 de mayo de 2015 2015:1-168.

[14] Bento-Coelho JL. Conforto sonoro em cidades - conceitos, instrumentos e 
estratégias. Congr. Iberoam. acústica FIA 2014, Valdivia-Chile: 2014, p. 112 .

[15] Liu J, Kang J, Behm H, Luo T. Effects of landscape on soundscape perception: Soundwalks in city parks. Landsc Urban Plan 2014;123:30-40. doi:10.1016/j.landurbplan.2013.12.003.

[16] Bento Coelho JL. A paisagem sonora como instrumento de design e engenharia em meio urbano. Proc. XXIII Encontro da Soc. Bras. Acústica, Salvador: 2010.

[17] H. Maslow A. A Theory of Human Motivation. New York: Harper and Row; 1954.

[18] Schomer P, Brown AL, De Coensel B, Genuit K, Gjestland T, Yong Jeon J, et al. On efforts to standardize a graphical description of the soundscape concept. Proceeding Inter-Noise 2010, 39th Int. Congr. Expo. Noise Control Eng., Lisbon: Portuguese Acoustical Society; 2010, p. 1-8.

[19] Schafer RM. The Tuning of the World. Random House; 1977. doi:10.2307/3345272.

[20] Truax B. Acoustic communication. New Jersey: Ablex Publishing Corporation; 1984.

[21] Truax B, Barrett GW. Soundscape in a context of acoustic and landscape ecology. Landsc Ecol 2011;26:1201-7. doi:10.1007/s10980-011-9644-9.

[22] Davies WJ, Adams MD, Bruce NS, Marselle M, Cain R, Jennings PA, et al. The Positive Soundscape Project: A synthesis of results from many disciplines. 38th Int. Congr. Expo. Noise Control Eng. 2009, INTER-NOISE 2009, vol. 1, Ottawa: 2009, p. 663-72.

[23] Davies WJ, Adams MD, Bruce NS, Cain R, Carlyle A, Cusack P, et al. Perception of soundscapes: An interdisciplinary approach. Appl Acoust 2013;74:224-31. doi:10.1016/j.apacoust.2012.05.010.

[24] Hermida L, Pavón I, Lobo Soares A, Bento-Coelho J. On the Person-Place Interaction and Its Relationship with the Responses/Outcomes of Listeners of Urban Soundscape (Compared Cases of Lisbon and Bogotá): Contextual and Semiotic Aspects. Int J Environ Res Public Health 2019;16:1-21. doi:10.3390/ijerph16040551.

[25] Adams M, Cox T, Moore G, Croxford B, Refaee M, Sharples S. Sustainable soundscapes: noise policy and the urban experience. Urban Stud 2006;43:2385-98. doi:10.1080/00420980600972504.

[26] Kogan P, Turra B, Arenas JP, Hinalaf M. A comprehensive methodology for the multidimensional and synchronic data collecting in soundscape. Sci Total Environ 2017;580:1068-77. doi:10.1016/j.scitotenv.2016.12.061.

[27] Jennings P, Cain R. A framework for improving urban soundscapes. Appl 
Acoust 2013;74:293-9. doi:10.1016/j.apacoust.2011.12.003.

[28] Kang J. From understanding to designing soundscapes. Front Archit Civ Eng China 2010;4:403-17. doi:10.1007/s11709-010-0091-5.

[29] Brown AL. Soundscape planning as a complement to environmental noise management. Proceeding Internoise 2014 - 43rd Int. Congr. Noise Control Eng. Improv. World Through Noise Control, Melbourne: 2014, p. 1-10.

[30] Bento Coelho JL, Remy N, Vogiatzis K. Urban Sound Planning in the City Centre of Thessaloniki. ICSV 2015 - 22 Int. Congr. Sound Vib., Florence: 2015, p. 12-6.

[31] Remy N, Vogiatzis K. Soundscape \& Land Uses Management As CompreHensive Environmental Protection Action Policy Tool Within the Strategic Environmental Noise Mapping in Greece. ICSV 2016 - 23rd Int. Congr. Sound Vib. From Anc. to Mod. Acoust., Athens: 2016, p. 1-8.

[32] International Standar Organization (ISO). 12913-1 Acoustics Soundscape - Part 1 : Definition and conceptual framework 2013.

[33] Brown AL, Gjestlan T, Dubois D. Acoustic Environments and soundscapes. In: Kang J, Schulte-Fortkamp B, editors. Soundscape Built Environ., 2016, p. $1-16$.

[34] Axelsson Ö, Nilsson ME. On sound source identification and taxonomy in soundscape research. Proceeding Inter-Noise 2010, 39th Int. Congr. Expo. Noise Control Eng., Lisbon: Portuguese Acoustical Society; 2010.

[35] Bento J-L. Approaches to Urban Soundscape Management, Planning and Design. In: Kang J, Schulte-Fortkamp B, editors. Soundscape Built Environ., CRC Press; 2016, p. 197-214.

[36] Rychtáriková M, Vermeir G. Soundscape categorization on the basis of objective acoustical parameters. Appl Acoust 2013;74:240-7. doi:10.1016/j.apacoust.2011.01.004.

[37] Genuit K, Fiebig A. Psychoacoustics and its benefit for the soundscape approach. Acta Acust United with Acust 2006;92:952-8.

[38] Hall DA, Irwin A, Edmondson-Jones M, Phillips S, Poxon JEW. An exploratory evaluation of perceptual, psychoacoustic and acoustical properties of urban soundscapes. Appl Acoust 2013;74:248-54. doi:10.1016/j.apacoust.2011.03.006.

[39] Maristany A, Recuero López M, Asencio Rivera C. Soundscape quality analysis by fuzzy logic: A field study in Cordoba, Argentina. Appl Acoust 2016;111:106-15. doi:10.1016/j.apacoust.2016.04.013.

[40] Szeremeta B, Zannin PHT. Analysis and evaluation of soundscapes in public parks through interviews and measurement of noise. Sci Total Environ 2009;407:6143-9. doi:10.1016/j.scitotenv.2009.08.039. 
[41] Rey Gozalo G, Trujillo Carmona J, Barrigón Morillas JM, Vílchez-Gómez R, Gómez Escobar V. Relationship between objective acoustic indices and subjective assessments for the quality of soundscapes. Appl Acoust 2015;97:1-10. doi:10.1016/j.apacoust.2015.03.020.

[42] Guillén J, López I. Importance of personal, attitudinal and contextual variables in the assessment of pleasantness of the urban sound environment. 19th Int. Congr. Acoust. ICA, Madrid: 2007, p. 1-6.

[43] Kawai K, Kojima T, Hirate K, Yasuoka M. Personal evaluation structure of environmental sounds: Experiments of subjective evaluation using subjects' own terms. J Sound Vib 2004;277:523-33. doi:10.1016/j.jsv.2004.03.013.

[44] López I, Guillén J. Calidad acústica urbana: influencia de las interacciones audiovisuales en la valoración del ambiente sonoro. Medio Ambient y Comport Hum 2005;6:101-17.

[45] Jeon JY, Lee PJ, Hong JY, Cabrera D. Non-auditory factors affecting urban soundscape evaluation. J Acoust Soc Am 2011;130:3761-70. doi:10.1121/1.3652902.

[46] Pheasant R, Horoshenkov K, Watts G, Barrett B. The acoustic and visual factors influencing the construction of tranquil space in urban and rural environments tranquil spaces-quiet places? J Acoust Soc Am 2008;123:1446-57. doi:10.1121/1.2831735.

[47] Liu J, Kang J, Luo T, Behm H. Landscape effects on soundscape experience in city parks. Sci Total Environ 2013;454-455:474-81. doi:10.1016/j.scitotenv.2013.03.038.

[48] Herranz-Pascual K, García I, Aspuru I, Díez I, Santander Á. Progress in the understanding of soundscape: objective variables and objectifiable criteria that predict acoustic comfort in urban places. Noise Mapp 2016;3:247-63. doi:10.1515/noise-2016-0017.

[49] Herranz-Pascual K, García I, Diez I, Santander A, Aspuru I. Analysis of Field Data to Describe the Effect of Context ( Acoustic and Non-Acoustic Factors ) on Urban Soundscape. Appl Sci 2017;7:1-18. doi:10.3390/app7020173.

[50] Hong JY, Jeon JY. Landscape and Urban Planning Influence of urban contexts on soundscape perceptions: A structural equation modeling approach. Landsc Urban Plan 2015;141:78-87. doi:10.1016/j.landurbplan.2015.05.004.

[51] Axelsson Ö, Nilsson ME, Berglund B. A principal components model of soundscape perception. J Acoust Soc Am 2010;128:2836-46. doi:10.1121/1.3493436. 
[52] Cain R, Jennings P, Poxon J. The development and application of the emotional dimensions of a soundscape. Appl Acoust 2013;74:232-9. doi:10.1016/j.apacoust.2011.11.006.

[53] Genuit K, Fiebig A. The measurement of soundscapes - Is it standardizable? Proceeding Internoise 2014 - 43rd Int. Congr. Noise Control Eng. Improv. World Through Noise Control, Melbourne: 2014, p. 9.

[54] Genuit K, Fiebig A. Human Hearing-Related Measurement and Analysis of Acoustics Environments: Requisite for Soundscape Investigations. In: Kang J, Schulte-Fortkamp B, editors. Soundscape Built Environ., CRC Press; 2016, p. 133-60.

[55] Rychtáriková M, Vermeir G, Domecka M. The application of the soundscape approach in the evaluation of the urban public spaces. J Acoust Soc Am 2008;123:3810. doi:10.1121/1.2935528.

[56] Rychtáriková M, Vermeir G. Acoustical Categorization of Urban Public Places by Clustering Method. In: Boone M, editor. NAG/DAGA Int. Conf. Acoust., Rotterdam: NAG/DAGA 2009; 2009.

[57] Deng Z, Kang J, Wang D, Liu A, Kang JZ. Linear multivariate evaluation models for spatial perception of soundscape. J Acoust Soc Am 2015;138:2860-70. doi:10.1121/1.4934272.

[58] International Standar Organization (ISO). ISO/TS 12913-2:2018. Acoustics - Soundscape - Part 2: Data collection and reporting requirements 2018:140.

[59] Herranz-Pascual K, Aspuru I, García I. Proposed conceptual model of environmental experience as framework to study the soundscape. Proc. Internoise 2010. Noise Sustain., Lisbon: 2010.

[60] Garcia I, Herranz-Pascual K, Aspuru I, Gutierrez L, Acero JA, Santander A. Indicators of Environmental Comfort Sensitive to Human Perception. In: Aletta F, Xiao J, editors. Handb. Res. Perception-Driven Approaches to Urban Assess. Des., 2018, p. 508-33. doi:10.4018/978-1-5225-36376.ch022.

[61] Pol E, Valera S, Vidal T. Psicología ambiental y procesos psicosociales. In: Morales F, Huici C, editors. Psicol. Soc., Madrid: 1999, p. 235-52.

[62] Romero VP, Brambilla G, Gabriele M Di, Gallo V, Maffei L. The influence of the soundscape on the tourists ' environmental quality perception. Proceeding Euronoise 2015, Maastrícht: 2015, p. 1535-40.

[63] Liu A, Liu F, Deng Z, Chen W. Relationship between soundscape and historical-cultural elements of Historical Areas in Beijing: A case study of Qianmen Avenue. Proceeding Internoise 2014 - 43rd Int. Congr. Noise Control Eng. Improv. World Through Noise Control, Melbourne: 2014, p. 


\section{$1-7$.}

[64] Hermida L, Lobo Soares AC, Pavón I, Bento Coelho JL. Assessing soundscape: Comparison between in situ and laboratory methodologies. Noise Mapp 2017;4:57-66. doi:10.1515/noise-2017-0004.

[65] Jeon JY, Hong JY, Lavandier C, Lafon J, Axelsson Ö, Hurtig M. A crossnational comparison in assessment of urban park soundscapes in France, Korea, and Sweden through laboratory experiments. Appl Acoust 2018;133. doi:10.1016/j.apacoust.2017.12.016.

[66] Kang J. Urban sound environment. Taylor \& Francis; 2007. doi:10.1007/s13398-014-0173-7.2.

[67] Payne SR, Davies WJ, Adams M. Research into the practical and policy applications of soundscape concepts and techniques in urban areas (NANR 200). 2009.

[68] Lobo Soares AC, Bento Coelho JL. Urban park soundscape in distinct sociocultural and geographical contexts. Noise Mapp 2016;3:232-46. doi:10.1515/noise-2016-0016.

[69] Zhang M, Kang J. Towards the evaluation, description, and creation of soundscapes in urban open spaces. Environ Plan B Plan Des 2007;34:6886. doi:10.1068/b31162.

[70] Bento JL. Conforto sonoro em cidades - conceitos, instrumentos e estratégias. Congr. Iberoam. acústica FIA 2014, Valdivia-Chile: 2014.

[71] Brambilla G, Gallo V, Asdrubali F, D'Alessandro F. The perceived quality of soundscape in three urban parks in Rome. J Acoust Soc Am 2013;134:832-9. doi:10.1121/1.4807811.

[72] Carles JL, Barrio IL, De Lucio JV. Sound influence on landscape values. Landsc Urban Plan 1999;43:191-200. doi:10.1016/S0169-2046(98)00112-1.

[73] Hume K, Ahtamad M. Physiological responses to and subjective estimates of soundscape elements. Appl Acoust 2013;74:275-81. doi:10.1016/j.apacoust.2011.10.009.

[74] Viollon S, Lavandier C, Drake C. Influence of visual setting on sound ratings in an urban environment. Appl Acoust 2002;63:493-511. doi:10.1016/S0003-682X(01)00053-6.

[75] Hong JY, Jeon JY. Designing sound and visual components for enhancement of urban soundscapes. J Acoust Soc Am 2013;134:2026-36. doi:10.1121/1.4817924.

[76] Axelsson Ö. Introducing soundscape. AESOP 26th Annu Congr 2012.

[77] Russell JA. A circumflex Model of Affect. J Pers Soc Psychol 1980;39:116178. doi:10.1037/h0077714.

[78] Jeon JY, Lee PJ, You J, Kang J. Perceptual assessment of quality of urban 
soundscapes with combined noise sources and water sounds. J Acoust Soc Am 2010;127:1357-66. doi:10.1121/1.3298437.

[79] Ando Y. Springer Series in Electrophysics Editors: Concert Hall Acoustics. vol. 17. Berlin: Springer; 1985.

[80] Ando Y, Cariani P. Auditory and visual sensations. London: Springer; 2009. doi:10.1007/b13253.

[81] Ando Y. Opera House Acoustics Based on Subjective Preference Theory. Kobe-Japan: Springer Japan; 2015. doi:10.1007/978-4-431-55423-3.

[82] Fujii K, Atagi J, Ando Y. Temporal and Spatial Factors of Traffic Noise and Its Annoyance. J Temporal Des Archit Environ 2002;2:33-41.

[83] Sato SI, Kitamura T, Ando Y. Annoyance of noise stimuli in relation to the spatial factors extracted from the interaural cross-correlation function. J Sound Vib 2004;277:511-21. doi:10.1016/j.jsv.2004.03.012. 


\section{CAPÍTULO 2}

\section{ANÁLISIS DE ASPECTOS ESPACIALES Y TEMPORALES EN LA EVALUACIÓN DE PAISAJES SONOROS: Adaptación e} implementación de parámetros acústicos derivados de las funciones de autocorrelación (ACF) y correlación cruzada interaural (IACF). 


\section{SEGÚNDO CAPÍTULO. ANÁlisis DE ASPECTOS ESPACIALES Y TEMPORALES EN LA EVALUACIÓN DE PAISAJES SONOROS: Adaptación e implementación de parámetros acústicos derivados de las funciones de autocorrelación (ACF) y correlación cruzada interaural (IACF).}

El desarrollo de este capítulo se centra en dos aspectos principales: 1) El análisis del impacto de las variaciones espaciales de las fuentes sonoras en el plano horizontal en la percepción de entornos acústicos en el estudio de paisajes sonoros y 2) La adaptación y aplicación de parámetros acústicos que mejoren la descripción de atributos perceptuales que indiquen dinamismo y variación de los entornos acústicos urbanos. Por lo tanto, las preguntas a las que se les quiere dar respuesta en este capítulo son:

- ¿Cuál es la influencia de la variación de las fuentes sonoras en el plano horizontal respecto a atributos perceptuales relacionados con la variabilidad de los paisajes sonoros?

- ¿Esta influencia puede reflejarse también en variaciones temporales energéticas?

- ¿Qué parámetros acústicos permiten la descripción de dichos comportamientos?

Para dar respuesta a estas preguntas este capítulo presenta la adaptación e implementación de parámetros acústicos asociados con las funciones de autocorrelación (ACF) y de correlación cruzada interaural (IACF) en la evaluación de entornos y paisajes sonoros. El capítulo dos se divide en cuatro partes: aspectos teóricos, aspectos metodológicos, resultados y discusión y conclusiones. En el abordaje teórico fueron estudiados parámetros asociados a las ACF e IACF según las teorías de Yoichi Ando, el modelo de evaluación subjetiva de paisajes sonoros de Östen Axelsson y aspectos descriptivos de paisajes sonoros según Lex Brown y Murray Schafer. En aspectos metodológicos se presenta la metodología usada en las pruebas desarrolladas en esta tesis a fin de determinar tanto la correlación entre parámetros acústicos derivados de las ACF e IACF y 
atributos perceptuales, así como la detección de diferencias estadísticamente significativas en paisajes sonoros según aspectos descriptivos y parámetros acústicos derivados de las ACF e IACF. Los resultados son divididos según parámetros binaurales y temporales. Finalmente se presenta la discusión y conclusiones, donde se discute la propuesta de evaluación de los entornos acústicos según los resultados y experiencias de esta tesis.

\subsection{Abordaje teórico de aspectos espaciales y temporales en la evaluación de paisajes sonoros.}

2.1.1 Uso de parámetros acústicos en el estudio de paisajes y entornos acústicos.

Como fue expuesto en el Capítulo I sección 1.5, para el estudio de paisajes y entornos acústicos, se han utilizado principalmente descriptores acústicos de tipo energético [1-4]. Niveles continuos equivalentes, valores máximos, percentiles 90 y 10 son algunos de los descriptores energéticos tradicionalmente usados. Sigue a continuación una explicación básica de ellos.

Nivel continuo equivalente (Leq): Representa la energía acústica promediada en el tiempo, y la norma ISO 1996-1 lo define como:

$$
L_{\text {Aeq } T}=10 \log \left[\frac{1}{T} \int_{T} \frac{p_{A}^{2}(t)}{p_{0}^{2}} d t\right] d B
$$

donde $\mathrm{p}_{\mathrm{A}}^{2}$ representa la presión acústica instantánea ponderada $\mathrm{A}$ durante el proceso de medición y $\mathrm{p}^{2}{ }_{0}$ es la presión acústica de referencia $(20 \mu \mathrm{pa})$ [5].

Nivel de presión sonora máximo ponderado en frecuencia y ponderado en el tiempo (Lmax): Según la ISO 1996-1 es el mayor nivel de presión sonora ponderado en frecuencia y ponderado en el tiempo durante un intervalo de tiempo determinado.

Nivel percentil: Nivel de presión sonora ponderado en frecuencia y tiempo superado en el N\% del periodo de tiempo considerado. Es común el uso de los percentiles 10, 50 y 90 en el estudio de acústica ambiental y laboral. 
Así mismo, también se utilizan descriptores como las diferencias de percentiles L10-L90 y el rango de SPL, que representan las variaciones del nivel de presión acústica respecto a valores percentiles y a valores ponderados.

Si bien estos parámetros acústicos han permitido describir de forma objetiva atributos perceptuales como confort acústico y/o molestia frente al ruido, en los estudios de paisajes sonoros la variedad de atributos perceptuales es mucho mayor. En la sección 1.5 del capítulo anterior fueron enumerados dos grandes grupos de atributos perceptuales: Placer-Confort y Actividad-Diversidad, los cuales representan atributos como placer, confort, actividad, cantidad de eventos, calma o caos [6-11]. Sin embargo, como se describió en la misma sección del capítulo anterior, los parámetros energéticos convencionales no describen satisfactoriamente algunos de estos atributos, principalmente aquellos que indiquen actividad, movimiento o variaciones de las fuentes sonoras. Teniendo en cuenta que los parámetros acústicos tradicionales representan valores de promediado temporal energético, valores máximos o mínimos o valores de percentiles, es sencillo comprender las bajas correlaciones obtenidas entre aspectos objetivos y subjetivos: son pocos los descriptores acústicos que permiten apreciar variaciones temporales de los entornos acústicos, pues fueron diseñados para identificar la cantidad de energía acústica presente en el entorno acústico.

Así mismo, los atributos relacionados con el grupo Actividad-Diversidad pueden indicar variaciones temporales energéticas, pero también pueden tener un alcance potencial en la actividad y movimiento de las fuentes en el plano horizontal. En ese sentido, además de parámetros monoaurales, es necesario profundizar en aspectos binaurales que permitan obtener información de aspectos espaciales de las fuentes sonoras y ayuden a responder preguntas como: ¿la fuente tiene una única ubicación en el espacio? ¿La fuente está en movimiento? ¿este movimiento afecta la respuesta de los oyentes?

Por lo anterior, en este apartado se analiza la utilidad de parámetros acústicos alternativos en la respuesta de las anteriores preguntas. 
2.1.2 Teoría de las ACF e IACF: Parámetros relacionados con comportamientos espaciales y temporales.

Según Yoichi Ando, el comportamiento temporal y espacial de los sonidos reviste una gran importancia, puesto que ofrece información relacionada con las características de las señales presentes dentro del campo sonoro, aportando además información de las fuentes generadoras de dichas señales y de los espacios en que estas se encuentran. Esta información es extraída tanto de las sensaciones temporales como de las sensaciones espaciales. Las sensaciones temporales conciernen a cualidades auditivas más directamente relacionadas con patrones temporales y retrasos de tiempo. Las sensaciones espaciales en cambio se obtienen de cualidades auditivas más directamente relacionadas con aspectos espaciales del sonido y del campo sonoro [12].

Para la evaluación de los campos sonoros y/o de las señales existentes dentro de dichos campos, Ando propone hacer uso de las funciones de autocorrelación y de correlación cruzada interaural. La función de autocorrelación (ACF) describe las señales monoaurales de cada uno de los dos oídos, mientras que la función de correlación cruzada interaural (IACF) describe la correlación entre las dos señales monuarales que alcanzan la entrada de los dos oídos. Si bien el campo de aplicación de estas funciones se ha dado principalmente en el ámbito de la acústica musical (detección de tonos y diferentes aspectos tonales) y de la acústica arquitectónica (análisis de ancho aparente de la fuente, ubicación en el plano horizontal y envolvimiento) se considera que algunos parámetros derivados de estas funciones pueden ser potencialmente útiles en la evaluación y estudio de paisajes y entornos sonoros. A continuación se presentan los descriptores asociados con las ACF e IACF.

\subsubsection{Factores Monoaurales: Función de Autocorrelación (ACF).}

La función de autocorrelación puede ser de largo término o de corta duración (también llamada móvil) según las necesidades o aplicaciones previstas. La función de autocorrelación de largo término $\Phi \mathrm{p}(\tau)$ se define por la siguiente expresión: 


$$
\Phi_{p}(\tau)=\lim _{T \rightarrow \infty} \frac{1}{2 T} \int_{-T}^{T} p^{\prime}(t) p^{\prime}(t+\tau) d t
$$

donde $\mathrm{p}^{\prime}=\mathrm{p}(\mathrm{t}){ }^{*} \mathrm{~s}(\mathrm{t}), \quad \mathrm{s}(\mathrm{t})$ representa la sensibilidad del oído. Para conveniencias prácticas, s(t) puede ser tomada como la respuesta al impulso (IR) de una red de ponderación A.

De la función de autocorrelación $\Phi \mathrm{p}(\tau)$ puede obtenerse la función de autocorrelación normalizada $\phi_{p}(t)$ definida por la siguiente expresión.

$$
\phi_{p}(t)=\Phi_{p}(\tau) / \Phi_{p}(0)
$$

donde $\Phi p(0)$ es la energía representada en el origen del retraso.

De $\phi_{p}(t)$ es posible estimar parámetros como el ancho de amplitud alrededor del origen del tiempo de retraso definido $\mathrm{W}_{\phi}(0)$, el retraso temporal del primer pico de la ACF $\tau 1$ y la amplitud del primer pico de la $\mathrm{ACF} \phi 1$ (ver figura 2-1). Estos parámetros se encuentran asociados principalmente con aspectos tímbricos y periódicos de los sonidos [13].

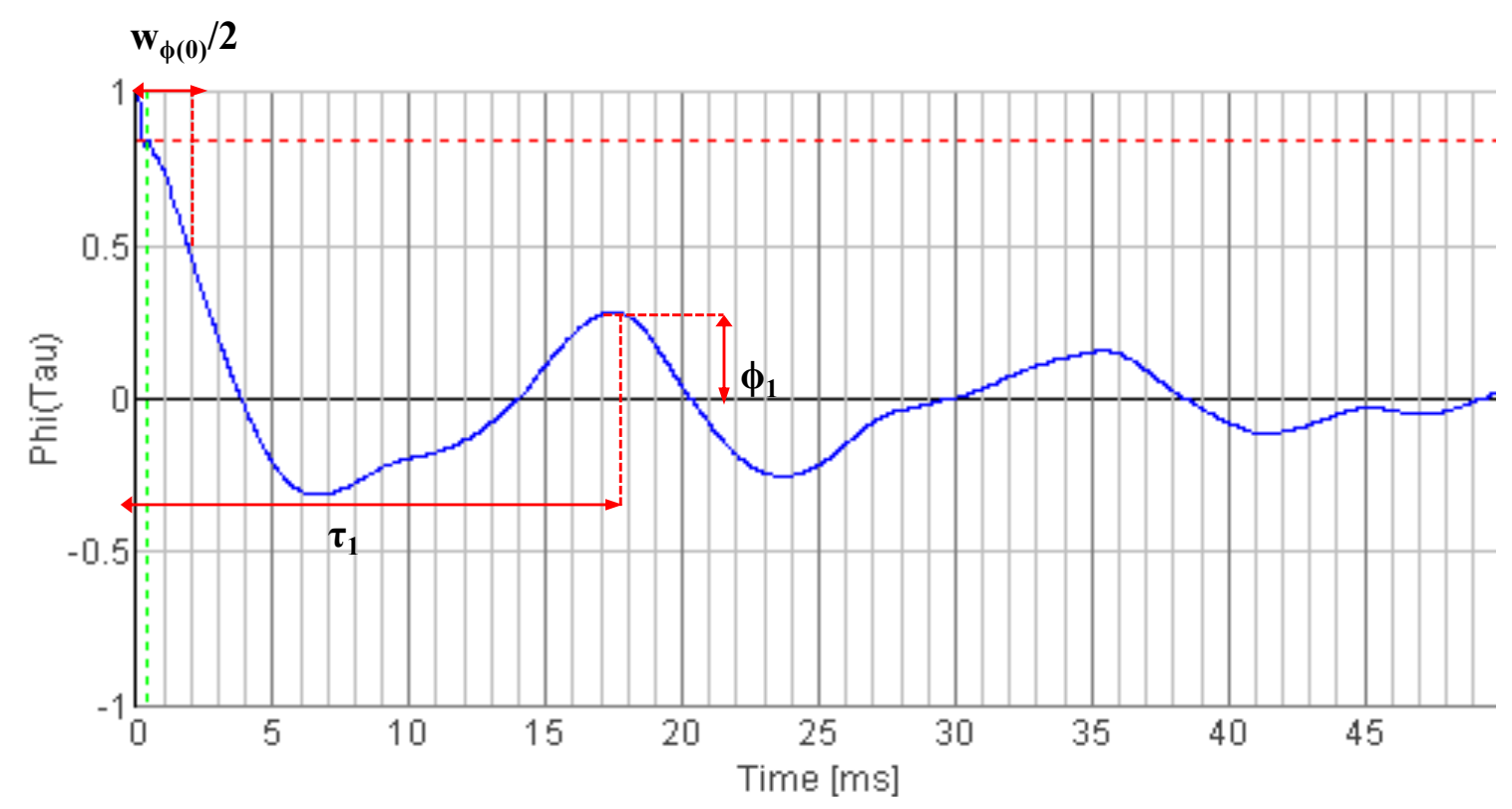

Figura 2-1. Factores temporales de $A C F W \varphi(0), \phi 1$ y $\tau 1$.

Al calcular la envolvente de $\phi p(\tau)$ definida como,

$$
\text { Env. ACF }=10 \log \left|\phi p_{(\tau)}\right|
$$

es posible calcular la duración efectiva de la envolvente normalizada de la $\mathrm{ACF} \tau_{\mathrm{e}}$. Esta se define como el tiempo de retraso que presenta la envolvente de 
$\mathrm{ACF}$ de $\phi \mathrm{p}(\tau)$ hasta decaer a su percentil 10 (figura 2-2). Teniendo en cuenta que se trata de una función de autocorrelación (que analiza la semejanza de una señal respecto a ella misma en función del tiempo), al tomar valores absolutos de $\phi \mathrm{p}(\tau)$ para señales sinusoidales puras se encontrará que la envolvente de ACF es plana, con una pendiente de cero, por lo que $\tau_{\mathrm{e}}$ tiende a infinito. Esto representa una característica repetitiva o de reverberación contenida en la propia fuente. Por lo tanto, $\tau_{\mathrm{e}}$ es una medida de coherencia temporal durante la cual la señal es autosimilar y menos degradada por reverberaciones [12]. Para el estudio de paisajes sonoros se considera que $\tau_{\mathrm{e}}$ puede llegar a ser de gran utilidad, pues permite describir numéricamente la semejanza de la señal con ella misma, lo que en algunos entornos sonoros con avenidas de alto flujo vehicular, plazas o parques podría ser un aspecto interesante a explorar.

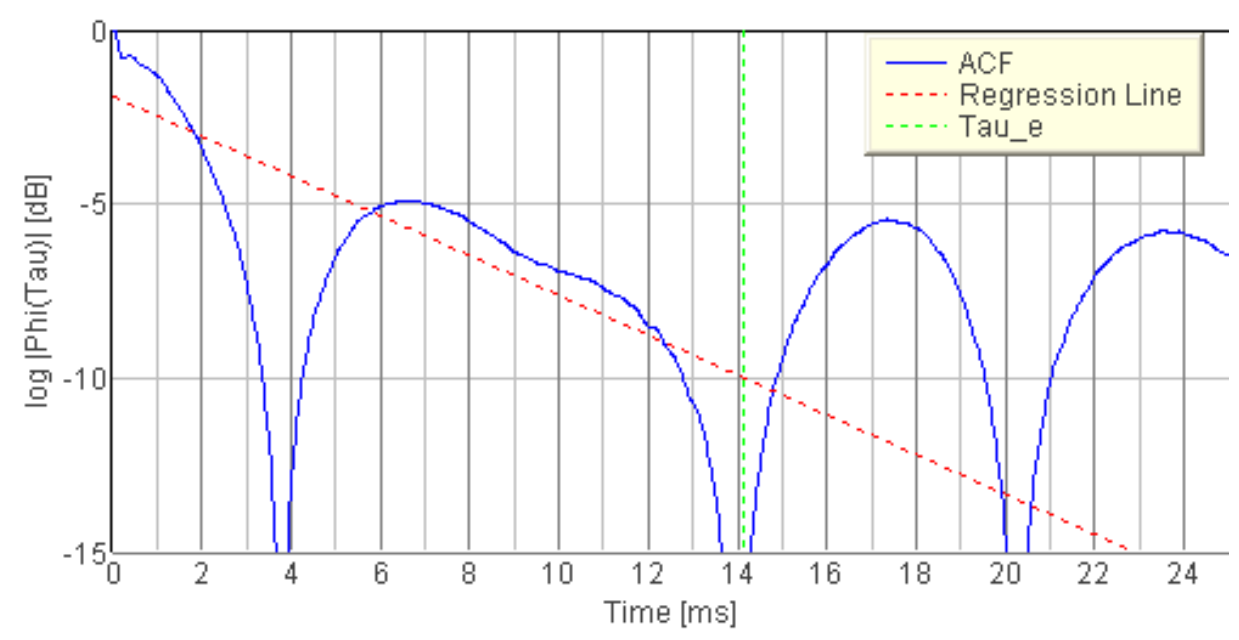

Figura 2-2. Duración efectiva de la envolvente normalizada de la ACF te

Por otra parte, pasando a la autocorrelación de corta duración o autocorrelación móvil, esta aparece debido a la necesidad de obtener información de señales de mayor duración, no solo de un evento sonoro sino de los múltiples eventos contenidos en periodos de tiempo largos. Para ello se establece una ventana de integración fija y un paso de desplazamiento como se presenta en la figura 2-3 [14]. 

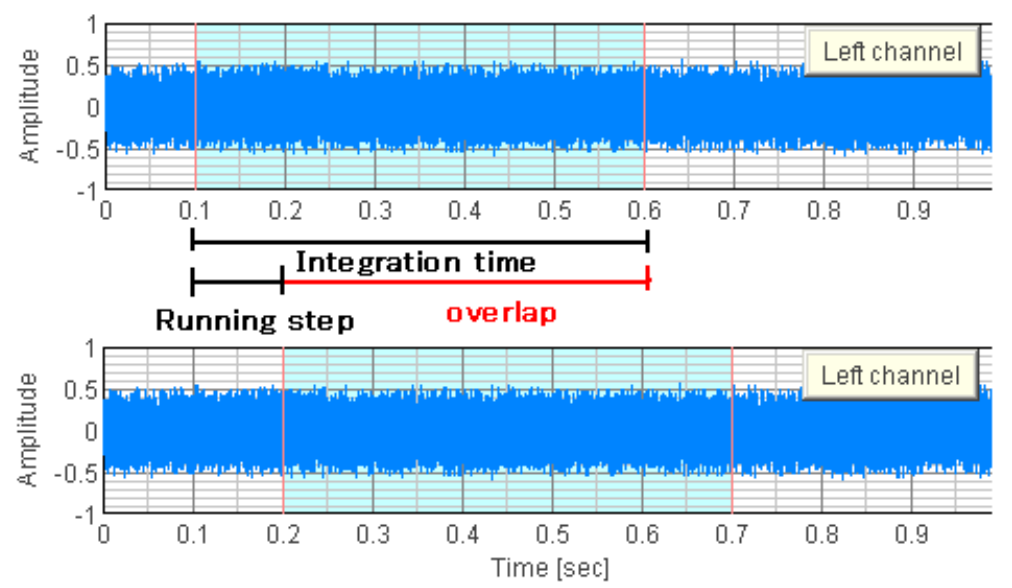

Figura 2-3. Función de autocorrelación móvil o de corta duración.

La ACF móvil se puede calcular de la siguiente forma:

$$
\begin{gathered}
\phi_{p}(\tau)=\phi_{p}(\tau ; t, T) \\
\phi_{p}(\tau)=\frac{\Phi_{p}(\tau ; t, T)}{\left[\Phi_{p}(0 ; t, T) \Phi_{p}(0 ; \tau+1, T)\right]^{1 / 2}}
\end{gathered}
$$

donde

$$
\Phi_{p}(\tau ; t, T)=\frac{1}{2} \int_{t-T}^{t+T} p^{\prime}(s) p^{\prime}(s+\tau) d s
$$

El resultado de este desplazamiento permite obtener diferentes valores de $\tau_{\mathrm{e}}$ respecto al tiempo en diferentes segmentos de la señal y realizar análisis del comportamiento de las duraciones efectivas a lo largo de todo el evento sonoro. De dicho análisis puede por lo tanto obtenerse $\tau_{\text {emin }}$, la duración efectiva mínima la cual se define como el valor mínimo en el desplazamiento de un ACF corto. El valor mínimo de un $\tau_{\mathrm{e}}$ se considera la parte más activa de la música y contiene información importante e influencia en la evaluación de la preferencia subjetiva de campos sonoros [15]. Se considera que la obtención de múltiples $\tau_{\mathrm{e}}$ en entornos acústicos pueden llegar a ofrecer información relacionada con la actividad o variación de fuentes sonoras, esto a partir del uso de diferentes descriptores estadísticos.

\subsubsection{Factores binaurales: Función de correlación cruzada IACF.}

Además de los factores temporales que pueden llegar a ser descritos por los indicadores relacionados con las funciones de ACF, bajo el enfoque de esta tesis se hace necesario el estudio de parámetros que permitan obtener información 
espacial de los entornos acústicos. Según Ando, para la localización e impresiones espaciales del campo sonoro, es clave la información que llega a los oídos izquierdo $\left(\mathrm{fl}_{(\mathrm{t})}\right)$ y derecho $\left(\mathrm{fr}_{(\mathrm{t})}\right)$, los cuales no son factores mutuamente independientes entre si, como tampoco lo son la diferencia interaural de intensidad y la diferencia interaural de tiempo. Las funciones $\mathrm{fl}(\mathrm{t})$ y $\mathrm{fr}(\mathrm{t})$ son definidas como:

$$
\begin{aligned}
& f_{l}(t)=p(t) * g_{l}(t) \\
& f_{r}(t)=p(t) * g_{r}(t)
\end{aligned}
$$

siendo $g_{1}$ y $g_{\mathrm{r}}$ la respuesta al impulso de los oídos izquierdo y derecho, que incluyen la respuesta al impulso relacionados con la cabeza.

Por lo anterior, buscando estudiar esta interdependencia, Ando propone la función de correlación cruzada interaural (IACF) $\Phi \operatorname{lr}(\tau)$ entre las funciones fl(t) y la fr(t) descrita por la siguiente expresión.

$$
\Phi_{l r}(\tau)=\lim _{T \rightarrow \infty} \frac{1}{2 T} \int_{-T}^{T} f^{\prime}{ }_{l}(t) f^{\prime}{ }_{r}(t+\tau) d t, \quad|\tau| \leq 1 m s
$$

siendo $f^{\prime} l(t)$ y f'r(t) las funciones obtenidas después de pasar las funciones $\mathrm{f}_{\mathrm{l}, \mathrm{r}}$ por la red de ponderación $\mathrm{A}$.

Así mismo puede obtenerse la función de correlación cruzada interaural normalizada $\phi \operatorname{lr}(\tau)$.

$$
\phi_{l r}(\tau)=\frac{\Phi_{l r}(\tau)}{\sqrt{\Phi_{l l}(0) \Phi_{r r}(0)}}
$$

donde $\Phi$ ll(0) y $\operatorname{Tr}(0)$ son las ACF para cada oído con $\tau=0$, y $\tau$ la el tiempo de retraso interaural posible dentro de $\pm 1 \mathrm{~ms}$.

Al discretizar las llegadas de las señales que llegan al oído, la correlación cruzada interaural normalizada se puede expresar como:

$$
\Phi_{l r}^{(N)}(\tau)=\frac{\sum_{n=0}^{N} A^{2} \Phi_{l r}^{(n)}(\tau)}{\sqrt{\sum_{n=0}^{N} A^{2} \Phi_{l l}^{(n)}(0) \sum_{n=0}^{N} A^{2} \Phi_{r r}^{(n)}(0)}}
$$

Uno de los parámetros binaurales más conocidos es el IACC, el cual representa la magnitud de la máxima correlación cruzada interaural y se define por: 


$$
I A C C=\left|\phi_{l r}(\tau)\right| \max
$$

para el máximo valor posible del retraso de tiempo interaural $|\tau| \leq 1 \mathrm{~ms}$. Según Ando, el IACC presenta una gran relación con la localización de fuentes sonoras en el plano horizontal [12].

Además del IACC y de la correlación cruzada interaural normalizada, de las IACF también puede obtenerse el retraso temporal interaural asociado al IACC, conocido como $\tau_{\text {IACC. }}$ El $\tau_{\text {IACC }}$ se estima a partir tiempo de retraso entre el tiempo de origen (0) y el tiempo donde se encuentra IACC (figura 2-4). Los valores que puede tomar el $\tau_{\text {IACC }}$ están entre 1 y -1 indicando la ubicación de la fuente en el plano horizontal (-1 izquierda y 1 derecha) [15].

Por último, otro de los descriptores asociados a la IACF es el WIACC: ancho del máximo pico de la IACF, es decir, el ancho del IACC. El WIACC es el intervalo del retraso temporal en un valor $\delta$ por debajo del IACC y está relacionado con el ancho aparente de la fuente y la localización espacial de las fuentes sonoras [16]. Este descriptor está definido por el tamaño del rango del retraso bajo el que el pico del IACF es menor del $90 \%$ del máximo valor $(\delta=0,1 * \mathrm{IACC})$. Una buena definición de la impresión direccional es percibida cuando se escucha un sonido con un pico agudo en el IACF con un bajo valor de $\mathrm{w}_{\mathrm{IACC}}$ [17]. La gráfica 2-4 presenta la IACF y sus parámetros asociados: IACC, $\tau_{\text {IACC }}$ y el wiACC.

Al igual que la $\mathrm{ACF}$, la IACF puede ser calculada en su categoría móvil, mediante el desplazamiento temporal de un periodo constante de integración respecto al tiempo. Se considera que información obtenida de la IACF móvil (y de sus parámetros asociados) es potencialmente útil en el estudio y evaluación de entornos y paisajes sonoros, pues permite apreciar las variaciones de la localización de fuentes sonoras en el espacio respecto al tiempo. 


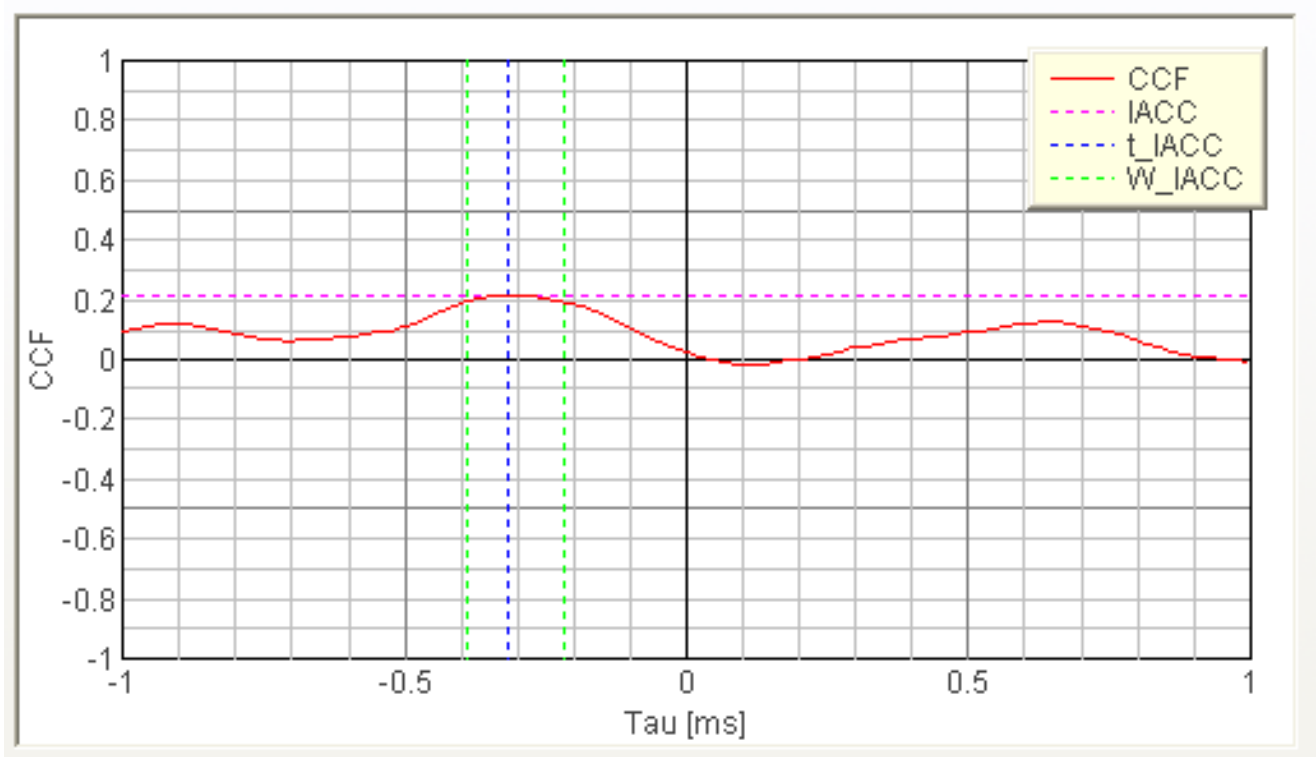

Figura 2-4. Factores espaciales relacionados con el IACF.

2.1.3 Evaluación subjetiva: Atributos perceptuales y modelos de evaluación subjetiva.

En la sección 1.5 fueron presentados diversos modelos de evaluación perceptual. Sin embargo, para el desarrollo de esta tesis se optó por trabajar con el modelo propuesto por Östen Axelsson para determinar respuestas de los oyentes en los entornos acústicos. El modelo de Axelsson está compuesto por un sistema cartesiano con dos ejes principales Eventful-Pleasant, el cual a su vez contiene otros seis atributos perceptuales (exciting-vibrant, calm, uneventful, boring (monotonous), unpleasant y stressful (chaotic)) tal como se presenta en la figura 2-5 $[6,18]$.

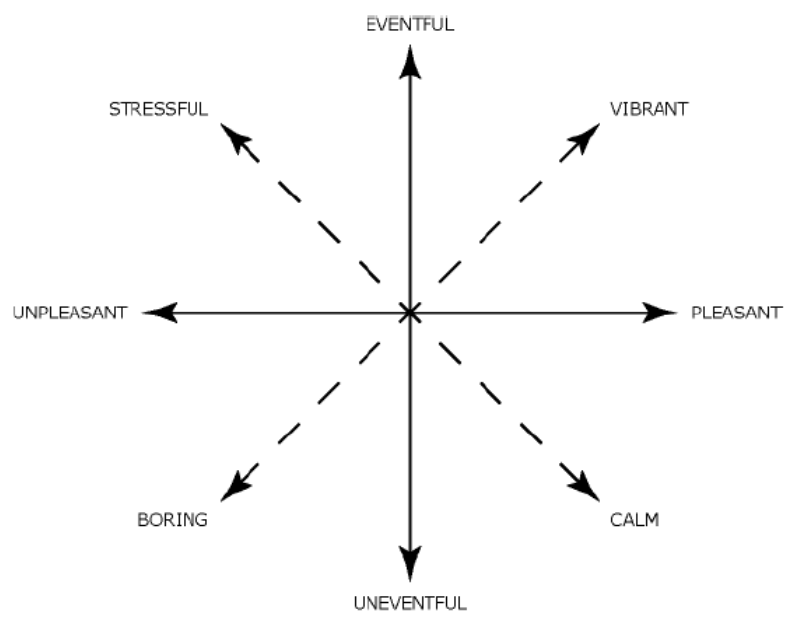

Figura 2-5. Modelo Bidimensional de atributos perceptuales de Axelsson [18]. 
A partir de los ocho atributos perceptuales presentados en la figura 2-5, es posible calcular los componentes principales Pleasantness y Eventfulness según las expresiones 14 y 15 [19-21].

$$
\begin{gathered}
\text { Pleasantness }=\frac{[(\sqrt{(2)} *(\text { pleasant }- \text { annoying }))+(\text { calm }- \text { chaotic })+(\text { exciting }- \text { monotonous })]}{[4+\sqrt{(8)}]} \\
\text { Eventfulness }=\frac{[(\sqrt{(2)} *(\text { eventful }- \text { unevent } f u l))-(\text { calm }- \text { chaotic })+(\text { exciting }- \text { monotonous })]}{[4+\sqrt{(8)}]}
\end{gathered}
$$

\subsubsection{Aspectos descriptivos de entornos acústicos.}

El aporte de Murray Schafer al estudio de paisajes sonoros no solo consiste en ser el primer investigador que acuño este término, sino que además, a través de sus diferentes proyectos a nivel mundial, ha propuesto formas alternas a la valoración cuantitativa para lograr descripciones de los paisajes sonoros. Es en este enfoque que Schafer presentó los conceptos de sonidos fundamentales y marcas sonoras para la descripción y entendimiento de los paisajes sonoros, lo cuales permiten tener un acercamiento perceptual a los paisajes sonoros. En este capítulo se retoman estos conceptos pues se consideran útiles para la apreciación y descripción de los entornos acústicos en estudio.

Según Schafer los sonidos fundamentales son definidos como aquellos sonidos que son escuchados continuamente o que están presentes con una constancia lo suficientemente fuerte como para formar un fondo contra lo cual los otros sonidos del paisaje sonoro van a contrastar. Estos sonidos según el mismo Schafer pueden no ser escuchados de forma consiente pues las personas se pueden acostumbrar a ellos, por lo que de alguna forma condicionan la percepción de los paisajes sonoros [22].

En lo que concierne a las marcas sonoras estas se refieren a aquellos sonidos que son propios de la comunidad o del lugar estudiado, o sea, aquellos sonidos que son únicos o representativos del lugar o la comunidad y que son especialmente percibidos.

En este capítulo se hará uso principalmente del concepto de sonido fundamental, pues permite describir el sonido dominante existente en cada uno de los paisajes sonoros estudiados. 
Así mismo, teniendo en cuenta la importancia los aspectos descriptivos en el estudio de paisajes sonoros, para la clasificación de sonidos fundamentales será tenido en cuenta el modelo de clasificación de fuentes sonoras de Lex Brown presentado en la figura 2-6. Dicho modelo presenta tres grandes clasificaciones: sonidos antroposónicos, sonidos geosónicos y sonidos biosónicos [23].

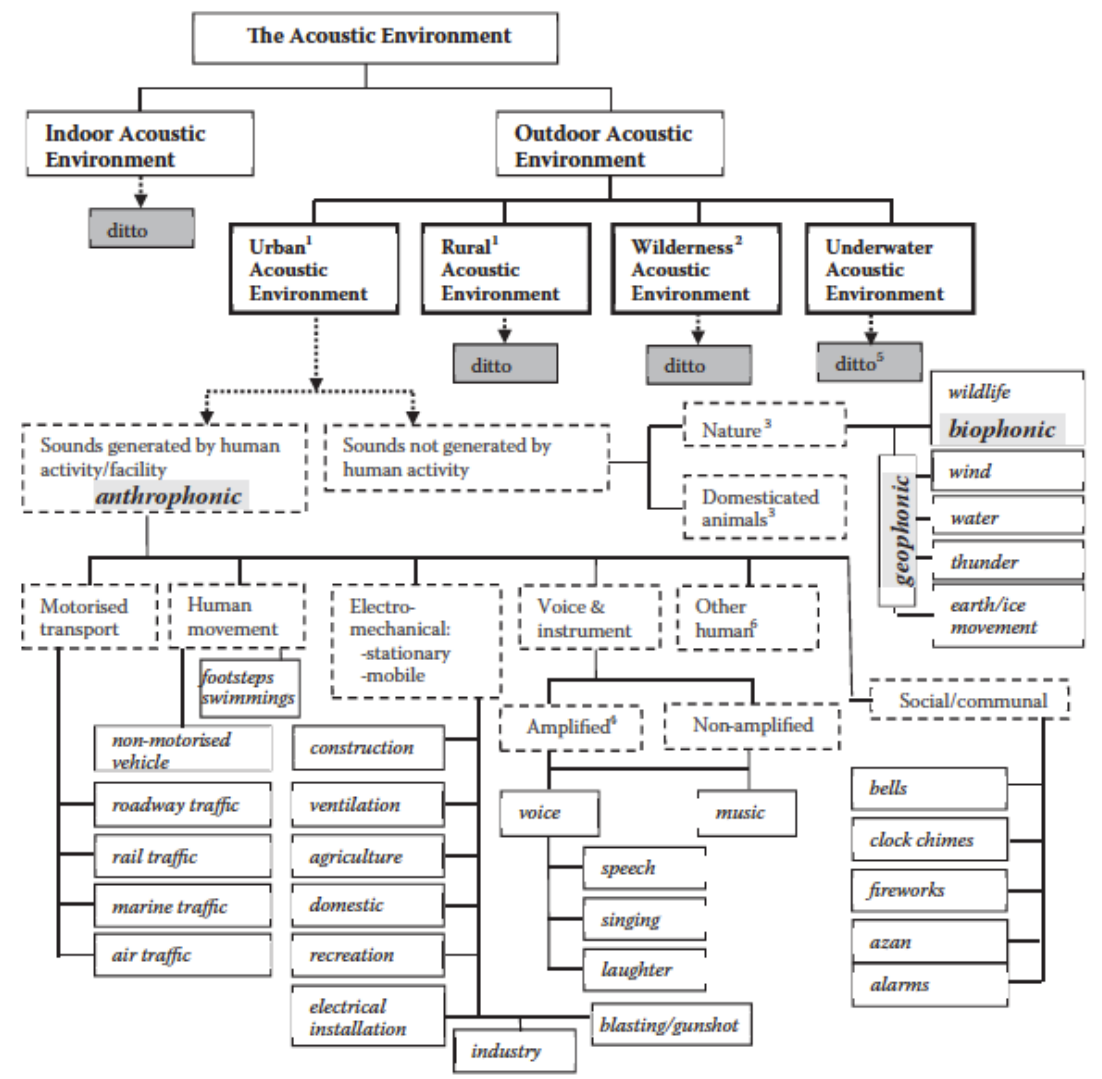

Figura 2-6. Clasificación de fuentes sonoras según modelo de Brown et al [23].

\subsection{Abordaje metodológico y pruebas realizadas.}

Con la finalidad de determinar la utilidad de parámetros acústicos relacionados con las funciones de autocorrelación y correlación cruzada interaural en el estudio de paisajes sonoros, fueron desarrolladas pruebas que involucran aspectos descriptivos, subjetivos y objetivos. Para ello fueron evaluados diez paisajes sonoros de las ciudades de Bogotá (Colombia) y Brasilia (Brasil). Respecto a los aspectos descriptivos, se analizaron los diferentes entornos acústicos según sus sonidos fundamentales y las diferentes fuentes sonoras que los componen. Así mismo se realizaron pruebas subjetivas en laboratorio aplicando el modelo de Axelsson y se usaron diferentes parámetros acústicos relacionados con las ACF e IACF. Se aplicaron pruebas de correlación de Spearman, así como 
pruebas Kruskal-Wallis para determinar la existencia de correlación entre parámetros objetivos y atributos perceptuales, así como para detectar diferencias estadísticamente significativas entre los diferentes entornos acústicos según su sonido fundamental y los parámetros acústicos. A continuación se proporciona una explicación más detallada del proceso desarrollado en estas pruebas.

\subsubsection{Lugares en estudio y grabación de entornos acústicos.}

Fueron escogidos cinco lugares de la ciudad de Bogotá-Colombia y cinco lugares de la ciudad de Brasilia-Brasil, analizando un total de diez paisajes sonoros. Para la selección de los lugares se tuvo en cuenta la diversidad de entornos acústicos que se pueden encontrar en una ciudad, por lo que en este estudio se consideraron centros de la ciudad, avenidas de alto flujo vehicular, plazas con presencia de fuente de agua, parques urbanos y centros de ferias y actividades comerciales. Los lugares de grabación en Brasilia fueron 1) el Eixo Rodoviario, (BRER, una de las principales avenidas de la ciudad), 2) Feria Torre de TV (BRFTV, una de las principales ferias artesanales en campo abierto de la ciudad), 3) Fuente de agua Torre de TV (BRFA, punto de encuentro y de referencia para los habitantes de la ciudad), 4) Parque Olhos D'agua (BRPOA, parque natural urbano ubicado en zona residencial) y 5) Centro de la ciudad (BRRO, punto de tránsito entre la rodoviaria metropolitana y centros comerciales). En la ciudad de Bogotá los lugares de grabación fueron la 6) Autopista norte estación Alcalá (BOAPA, importante autopista de la ciudad), 7) la Calle 19 con Cra. 7 (BOC197, centro de la ciudad, punto de tráfico vehicular y comercio), 8) la fuente de agua edificio Tequendama (BOFAET, plazoleta con fuente de agua), 9) la Plaza de Lourdes (BOPL, una de las plazas más representativas de la ciudad de Bogotá) y 10) el Parque Simón Bolívar (BOPSB, uno de los parques urbanos más grandes de América latina con 113ha). Para mayor información de los lugares en estudio ver Anexo 1.

A fin de no perturbar el desarrollo de actividades dentro de los diferentes lugares, ni llamar la atención de los usuarios de estos espacios con elementos ajenos a su cotidianidad (caso de cabezas binaurales), se usó un sistema de grabación binaural portátil conformado por grabadora Zoom H4N y micrófonos binaurales roland cs 10em (especificaciones técnicas presentadas en el Anexo 2). El control de ganancia del sistema es de paso digital, por lo que se aseguró un 
control cuidadoso de los niveles de la grabación según se expondrá en la sección 2.8. La frecuencia de muestreo de las grabaciones fue de $48000 \mathrm{~Hz}$ a una resolución de 24 bits. Los registros fueron realizados en puntos fijos paralelo a las principales fuentes sonoras de cada lugar. Los tiempos de grabación para cada punto fueron de 15 minutos.

2.2.2 Evaluación objetiva: parámetros binaurales, temporales y energéticos.

En el análisis de los aspectos temporales y espaciales se utilizaron las funciones de autocorrelación (ACF) y correlación cruzada (IACF) en sus funciones móviles y de largo término. Del total de 15 minutos de grabación para cada entorno acústico, fueron seleccionados para el procesamiento de señales 30 segundos de audio, teniendo en cuenta que cada fragmento representara actividades y prácticas desarrolladas cotidianamente de cada lugar. Así mismo para la longitud de cada fragmento de audio se consideraron trabajos previos que indican tiempos entre 6 segundos y 1 minuto de duración [2,6-8,24-27]. Los parámetros acústicos obtenidos de las ACF e IACF de larga duración tenidos en cuenta en esta tesis fueron el tiempo efectivo de duración $\tau_{\mathrm{e}} \mathrm{y}$ la correlación cruzada interaural (IACC).

Para las ACF e IACF móviles fueron analizadas las señales usando ponderaciones A y Z, obteniendo el tiempo efectivo de duración $\tau_{\mathrm{e}}$, la correlación cruzada interaural (IACC), el retraso temporal interaural $\left(\tau_{\text {IACC }}\right)$ y el ancho de IACC (WIACC). Debido a que en las funciones móviles las señales se fragmentan, es posible obtener múltiples valores de $\tau_{\mathrm{e}}$, IACC, $\tau_{\mathrm{IACC}} \mathrm{y} \mathrm{w}_{\mathrm{IACC}}$, por lo que es necesario el uso de diferentes descriptores estadísticos como medias, medianas, máximos, mínimos, rangos, percentiles 90 y 10 y diferencia de percentiles a fin de poder analizar estos parámetros. El intervalo de desplazamiento para las ACF e IACF móviles usado en este estudio fue de $100 \mathrm{~ms}$, con una ventana temporal de 2s. La tabla 2-1 presenta un resumen de los parámetros objetivos usados, así como los descriptores estadísticos empleados.

Finalmente, para tener un parámetro acústico tradicional, en este estudio se consideró el Nivel Continuo Equivalente con poderación A y Z. Este parámetro es uno de los más populares en el estudio de paisajes sonoros, presentando correlaciones considerables con el grupo de atributos perceptuales Placer-Confort $[6,28-30]$. 
Tabla 2-1. Parámetros acústicos objetivos usados para el análisis espacial y temporal de entornos acústicos.

\begin{tabular}{|c|c|c|c|c|}
\hline $\begin{array}{l}\text { Tipo de } \\
\text { análisis }\end{array}$ & Función general & Parámetros de análisis & $\begin{array}{l}\text { Descriptor } \\
\text { estadístico }\end{array}$ & Uso en este estudio \\
\hline \multirow[b]{2}{*}{ Espacial } & $\begin{array}{l}\text { IACF largo término: } \\
\Phi_{l r}(\tau)=\lim _{T \rightarrow \infty} \frac{1}{2 T} \int_{-T}^{T} f_{l}^{\prime}(t) f^{\prime}{ }_{r}(t+\tau) d t \\
|\tau| \leq 1 m s \\
\qquad f_{l}(t)=p(t) * g_{l}(t) \\
f_{r}(t)=p(t) * g_{r}(t) \\
\text { IACF normalizada: } \\
\qquad \phi_{l r}(\tau)=\frac{\Phi_{l r}(\tau)}{\sqrt{\Phi_{l l}(0) \Phi_{r r}(0)}}\end{array}$ & $\begin{array}{l}\text { IACC: Es la magnitud de } \\
\text { la máxima correlación } \\
\text { cruzada interaural. Se } \\
\text { expresa como: } \\
\quad I A C C=\left|\phi_{l r}(\tau)\right| \max \\
\text { para el máximo valor } \\
\text { posible del retraso de } \\
\text { tiempo interaural }|\tau| \leq 1 \mathrm{~ms} .\end{array}$ & $\begin{array}{l}\text { Valor global de } \\
\text { IACC }\end{array}$ & $\begin{array}{l}\text { Análisis de la semejanza entre las señales que } \\
\text { llegan al oído izquierdo y derecho del entorno } \\
\text { sonoro urbano. }\end{array}$ \\
\hline & $\begin{array}{l}\text { IACF móvil: } \\
\qquad \phi_{l r}(\tau)=\phi_{l r}(\tau ; t, T) \\
\phi_{l r}(\tau)=\frac{\Phi_{l r}(\tau ; t, T)}{\left[\Phi_{l r}(0 ; t, T) \Phi_{l r}(0 ; \tau+1, T)\right]^{1 / 2}}\end{array}$ & $\begin{array}{l}\text { IACC: A partir del } \\
\text { desplazamiento temporal de } \\
\text { la IACF en la señal de } \\
\text { análisis, se obtienen } \\
\text { múltiples IACC de los } \\
\text { diferentes segmentos de la } \\
\text { señal. } \\
\\
\tau_{\text {IACC: }} \text { Se calcula a partir del } \\
\text { tiempo de retraso entre el } \\
\text { tiempo de origen (0) y el } \\
\text { tiempo donde se encuentra } \\
\text { IACC. Los valores que } \\
\text { puede tomar el } \tau_{\text {IACC }} \text { están } \\
\text { entre } 1 \text { y -1. }\end{array}$ & $\begin{array}{l}\text { Media, } \\
\text { mediana, } \\
\text { mínimos, } \\
\text { máximos, } \\
\text { rangos, } \\
\text { percentiles } 90 \text { y } \\
10 \text { así como la } \\
\text { diferencia entre } \\
\text { percentiles p90 } \\
\text { y p10. } \\
\text { Rango y las } \\
\text { diferencias de } \\
\text { percentiles } 90- \\
10 \text { de } \tau_{\text {IACC }}\end{array}$ & $\begin{array}{l}\text { Dado que el IACC es uno de los principales } \\
\text { parámetros para la localización de fuentes en el } \\
\text { plano horizontal [12], a partir de la } \\
\text { segmentación del audio y de la obtención de } \\
\text { múltiples IACC, se pretende analizar la } \\
\text { correlación entre el comportamiento del IACC } \\
\text { en sus diferentes estadísticos descriptivos y } \\
\text { diferentes atributos perceptuales. } \\
\tau_{\text {IACC está relacionada con la ubicación de sonido }} \\
\text { en el plano horizontal [15]. En este trabajo los } \\
\text { descriptores estadísticos rango y diferencia de } \\
\text { percentiles se usan para analizar la variabilidad } \\
\text { de las ubicaciones de las fuentes sonoras en el } \\
\text { espacio. }\end{array}$ \\
\hline
\end{tabular}




\begin{tabular}{|c|c|c|c|c|}
\hline & & $\begin{array}{l}\text { wIACC: es el intervalo del } \\
\text { retardo temporal en un } \\
\text { valor } \delta \text { por debajo del } \\
\text { IACC. Este parámetro está } \\
\text { definido por el tamaño del } \\
\text { rango del retraso bajo el que } \\
\text { el pico del IACF es menor } \\
\text { del } 90 \% \text { del máximo valor } \\
\left(\delta=0,1^{*} \text { IACC }\right)[31] \text {. }\end{array}$ & $\begin{array}{l}\text { Rango y las } \\
\text { diferencias de } \\
\text { percentiles } 90- \\
10 \text { de WIACC }\end{array}$ & $\begin{array}{l}\text { wIACC: Ofrece información relacionada con } \\
\text { percepciones espaciales como localización y } \\
\text { claridad espacial en el plano horizontal, el } \\
\text { ancho aparente de la fuente (término aplicado } \\
\text { principalmente en el campo de acústica } \\
\text { arquitectónica) [16]. En este estudio los } \\
\text { descriptores estadísticos Rango y diferencia de } \\
\text { percentiles } 90-10 \text { se usan para analizar la } \\
\text { variabilidad de las ubicaciones de las fuentes } \\
\text { sonoras en el espacio. }\end{array}$ \\
\hline \multirow[b]{2}{*}{ Temporal } & $\begin{array}{l}\text { ACF de largo término: } \\
\qquad \boldsymbol{\Phi}_{\boldsymbol{p}}(\boldsymbol{\tau})=\lim _{\boldsymbol{T} \rightarrow \infty} \frac{\mathbf{1}}{\mathbf{2} \boldsymbol{T}} \int_{-\boldsymbol{T}}^{\boldsymbol{T}} \boldsymbol{p}^{\prime}(\boldsymbol{t}) \boldsymbol{p}^{\prime}(\boldsymbol{t}+\boldsymbol{\tau}) \boldsymbol{d} \boldsymbol{t} \\
\text { ACF normalizada: } \\
\qquad \phi_{p}(\tau)=\Phi_{p}(\tau) / \Phi_{p}(0)\end{array}$ & $\begin{array}{l}\tau_{\mathrm{e}} \text { : Se define como el tiempo } \\
\text { de retardo del percentil } 10 \\
\text { de la ACF normalizada y } \\
\text { representa una característica } \\
\text { repetitiva o de reverberación } \\
\text { contenida en la propia } \\
\text { fuente }[12] .\end{array}$ & $\begin{array}{l}\text { Valor global de } \\
\tau_{\mathrm{e}}\end{array}$ & $\begin{array}{l}\text { Estudio de la autosemejanza de una señal a } \\
\text { partir de la variación de la evolución temporal } \\
\text { de entornos sonoros. }\end{array}$ \\
\hline & $\begin{array}{l}\text { ACF de corto término o móvil: } \\
\qquad \phi_{p}(\tau)=\phi_{p}(\tau ; t, T) \\
\phi_{p}(\tau ; t, T) \\
\text { donde } \\
\left.\qquad \Phi_{p}(\tau ; t, T)=\frac{1}{2} \int_{t-T}^{t+T} p_{p}(0 ; t, T) \Phi_{p}(0 ; \tau+1, T)\right]^{1 / 2}\end{array}$ & $\begin{array}{l}\tau_{\mathrm{e}} \text { : Se obtiene a partir del } \\
\text { desplazamiento temporal de } \\
\text { la ACF en la señal de } \\
\text { análisis }[32] . \text { El } \\
\text { desplazamiento temporal } \\
\text { ofrece múltiples valores de } \\
\tau_{\mathrm{e}} \text { en el transcurso del } \\
\text { tiempo en diferentes } \\
\text { segmentos de la señal }\end{array}$ & $\begin{array}{l}\text { Media, } \\
\text { mediana, } \\
\text { mínimos, } \\
\text { máximos, } \\
\text { rangos, } \\
\text { percentiles } 90 \text { y } \\
10 \text { así como la } \\
\text { diferencia entre } \\
\text { percentiles p90 } \\
\text { y p10. }\end{array}$ & $\begin{array}{l}\text { Estudio de la variación de la autosemejanza de } \\
\text { señales de mayor duración. Permite apreciar la } \\
\text { variación de los diferentes eventos sonoros que } \\
\text { se presentan en diferentes segmentos de las } \\
\text { señales en estudio. }\end{array}$ \\
\hline
\end{tabular}




\subsubsection{Aspectos descriptivos y evaluación subjetiva de los paisajes sonoros.}

El análisis descriptivo de cada entorno sonoro se basó en el modelo de clasificación de fuentes sonoras propuesto por Brown [23], incluyendo también una descripción general de cada uno de los lugares en estudio. Así mismo, dentro de los aspectos descriptivos fueron tenidos en cuenta los sonidos fundamentales de cada paisaje sonoro. Para determinar el sonido fundamental de los diferentes paisajes sonoros en estudio, se realizaron pruebas de evaluación subjetiva en laboratorio a un panel de 25 personas. A los participantes se les presentó un listado de sonidos y se le pidió que indicaran cuan dominante percibían cada uno de ellos para cada fragmento de audio [33]. Las opciones de respuesta eran "no dominante", "poco dominante", "moderadamente dominante", "muy dominante" y "totalmente dominante", donde cada una de estas opciones correspondía a valores de $-1,-0.5,0,0.5$ y 1 respectivamente. Los sonidos fundamentales de cada lugar fueron asociados a fuentes sonoras detectadas de por parte del investigador en visitas de reconocimiento realizadas previo al desarrollo de las pruebas. Las fuentes sonoras fueron agrupadas según las cuatro categorías propuestas por Brown: Antroposónico, Geosónico y Biosónico. Las condiciones bajo las cuales fue desarrollada dicha evaluación se describen a continuación.

Para el diseño de la prueba subjetiva de laboratorio se tuvieron en cuenta diferentes recomendaciones provenientes del campo de evaluación de calidad sonora de productos relacionadas con número de evaluadores, tipos de pruebas y tiempos totales de evaluación. En las evaluaciones de calidad sonora el número de evaluadores depende del grado de entrenamiento auditivo que tengan los sujetos de prueba, de su relación con el objeto en estudio y de la complejidad del tipo de prueba [34,35]. Si las pruebas son muy complejas se recomienda usar un conjunto de 5 o 10 expertos con mucho entrenamiento y experiencia previa, si las pruebas requieren un alto grado de relación con el sonido en estudio se recomiendan entre 25 y 75 sujetos de pruebas y si simplemente se requieren oyentes con un bajo grado de relación con los productos se deben tener entre 75 y 100 sujetos.

La prueba de laboratorio aplicada en este estudio fue realizada a 25 oyentes, quienes contaban con conocimientos en el campo de acústica (estudiantes 
de mínimo 3 años de estudio en el campo de Ingeniería de Sonido y profesores con amplio entrenamiento auditivo).

Los tiempos máximos de evaluación subjetiva recomendados para evitar fatiga en los oyentes deben ser de entre 30 y 45 minutos. En este caso a los oyentes les fueron presentados 10 fragmentos de audio de 30 segundos de duración correspondientes a los 10 lugares en estudio, por lo que las pruebas presentaban un tiempo máximo de duración de 20 minutos, esto considerando que los tiempos recomendados para pruebas de calidad sonora son de 30 a 45 minutos para no generar fatiga en los oyentes [35-37]. El orden de reproducción era aleatorio para cada uno de los oyentes, a fin de evitar sesgos por orden de reproducción. El método de reproducción fue el "self-paced", donde el oyente pasaba cada fragmento de audio, respondía las preguntas de la prueba y pasaba al siguiente audio. Por lo anterior, la separación entre cada audio dependía directamente de la velocidad de respuesta de cada oyente. Fueron usados auriculares en el proceso de escucha y los sistemas de grabación y reproducción fueron calibrados utilizando un calibrador tipo I y una cabeza binaural $01 \mathrm{~dB}$ tal como se puede apreciar en la figura 2-7, esto con el fin de asegurar que los niveles de presión sonora presentados a los oyentes tuvieran la máxima similitud a los existentes en los entornos acústicos in situ en estudio (mayores especificaciones de las pruebas desarrolladas en el sistema de reproducción se presentan en el Anexo 3). Las pruebas fueron desarrolladas en los estudios de grabación de la Universidad San Buenaventura, sede Bogotá. El perfil de ruido del lugar de pruebas, acorde a medición realizada según ANSI/ASA S12.2 2008 [38], corresponde a NC 25.

Así mismo, para la evaluación subjetiva de respuestas a corto plazo, fue considerada la evaluación general del entorno sonoro así como los ocho atributos perceptuales del modelo de Axelsson [6]: pleasant, chaotic, exciting, uneventful, calm, annoying, eventful, and monotonous. Estos atributos permitieron la estimación de los componentes Pleasantness y Eventfulness del modolo bidimensional orto-normalizado del modelo de Axelsson [21]. Estos atributos fueron traducidos al español y adaptados al español latinoamericano. Los resultados de la traducción al español son los siguientes: Agradable, Caótico, excitante/vibrante, Uniforme (sin eventos), Calmo, desagradable, Agitado/variado, Monótono. Para la evaluación de cada uno de los atributos fue 
usada la técnica de escala de respuestas semántica (response escale) preguntando a los sujetos "¿en que extensión considera que los siguientes 8 factores describen el ambiente sonoro escuchado?", presentando cinco niveles de respuesta: de acuerdo completamente, de acuerdo parcialmente, ni de acuerdo ni en desacuerdo, parcialmente en desacuerdo y totalmente en desacuerdo. Para el procesamiento de datos, cada uno de estos niveles tomaron valores entre \pm 1 a un paso de 0.5 , siendo 1 concuerdo completamente y -1 totalmente en desacuerdo.

De esta forma la prueba subjetiva se encuentra dividida en cuatro grandes secciones:

- perfil general del oyente.

- aspectos descriptivos (evaluación de sonido fundamental y marca sonora)

- respuestas a corto plazo (atributos perceptuales según modelo de Axelsson)

- salidas a largo plazo (información relacionada con el uso y significado)

El esquema general de la prueba se puede apreciar en la figura 2-8. El anexo 4 presenta la herramienta de evaluación desarrollada.

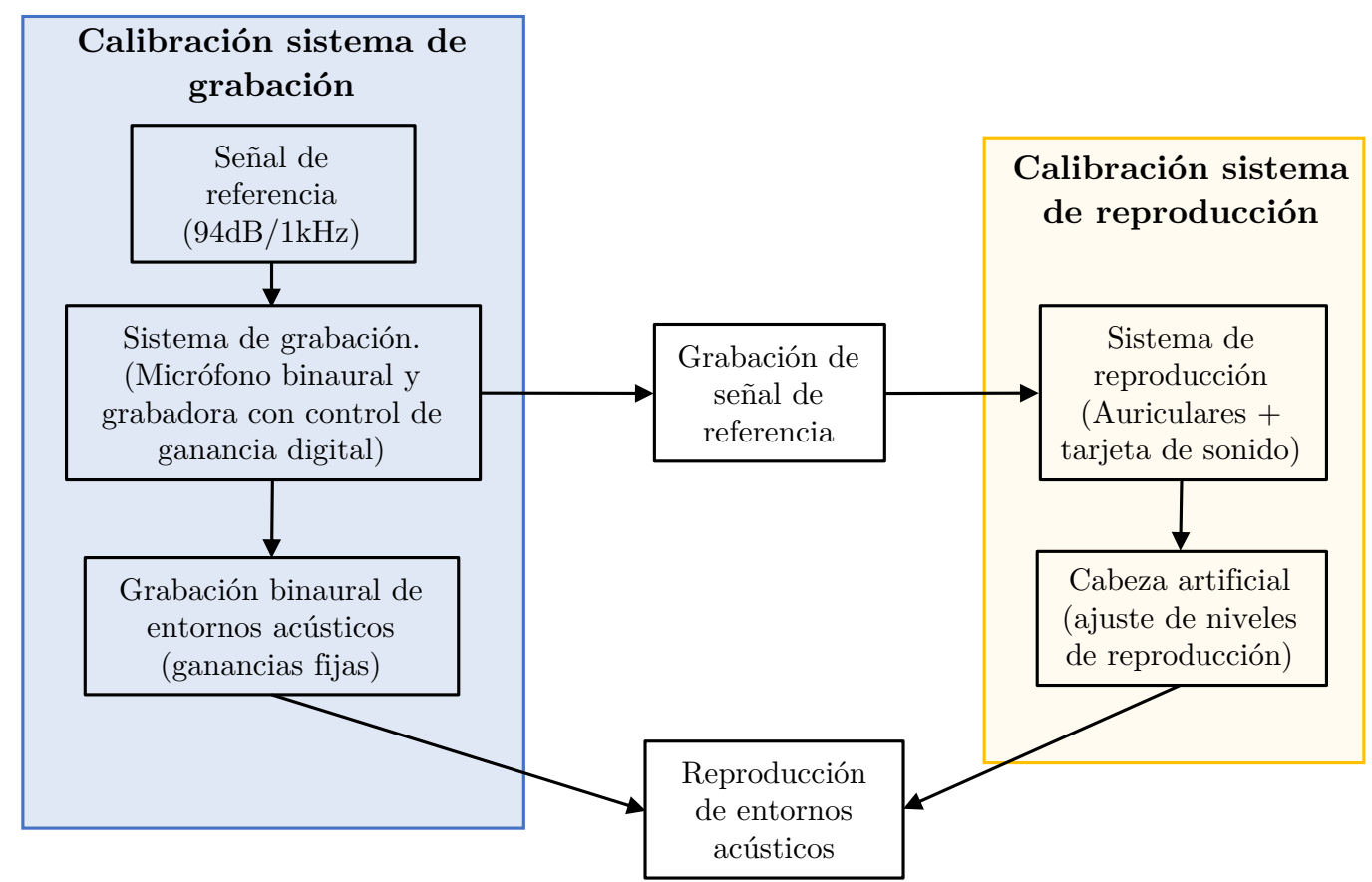

Figura 2-7. Diagrama de proceso de calibración para la grabación y reproducción de ambientes sonoros. 


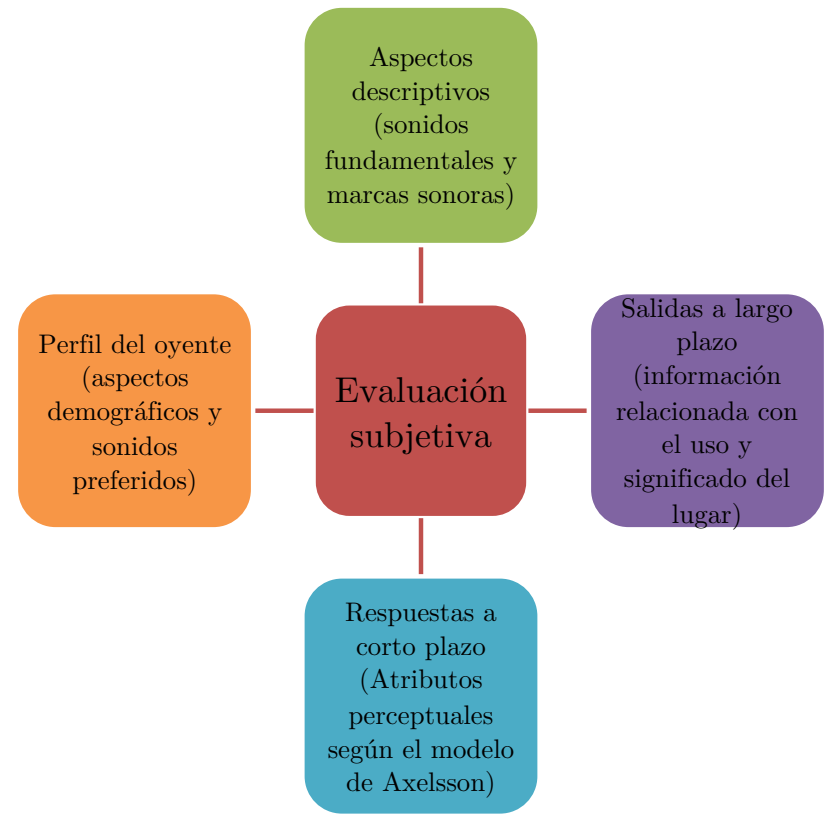

Figura 2-8. Aspectos generales del proceso de evaluación subjetiva.

2.2.4 Procesado de datos y pruebas estadísticas.

Tanto para el caso de los datos obtenidos en las pruebas objetivas como en las pruebas subjetivas, fueron aplicadas las pruebas de normalidad de ShapiroWilk, lo que permitió determinar que los datos no presentaban distribuciones normales. Por esta razón fueron aplicadas pruebas no paramétricas (pruebas de Kruskal-Wallis y coeficiente de correlación de Spearman) con el fin de: 1) determinar dependencias estadísticas entre los sonidos fundamentales y los parámetros acústicos derivados de las ACF e IACF móvil y de largo término y 2) determinar la correlación entre parámetros objetivos derivados de las ACF e IACF y los atributos perceptuales. Se utilizaron las medias como medida de tendencia central para la representación de los atributos perceptuales según el modelo de Axelsson.

\subsection{Resultados.}

\subsubsection{Aspectos descriptivos}

La tabla 2-2 presenta una descripción general de los entornos acústicos en estudio, las fuentes sonoras, así como los sonidos fundamentales y la categoría principal y secundaria a la que pertenecen dichos sonidos fundamentales. Según los oyentes, 4 lugares presentaron como sonido fundamental al tráfico vehicular 
(sonidos de origen antroposónico), mientras que otros 4 presentaron como sonido fundamental sonidos de agua, pájaros y naturaleza en general (sonidos geosónicos y biosónicos) y otros dos presentaron como sonido fundamental las voces de personas (sonido antroposónico). Lugares con la mayor diversidad de fuentes sonoras fueron los centros de ciudades (BOC197 y BRRO) y plazas con actividades comerciales (BRFTV y BRPL).

Tabla 2-2. Descripción, listado de sonidos, sonidos fundamentales y categorías principal y secundaria a la que pertenecen los sonidos fundamentales. En color violeta lugares de Bogotá, en color azul lugares de Brasil.

\begin{tabular}{|c|c|c|c|c|c|}
\hline Lugar & $\begin{array}{c}\text { Sonido } \\
\text { fundamental }\end{array}$ & $\begin{array}{c}\text { Fuente Sonora } \\
\text { asociada al } \\
\text { sonido } \\
\text { fundamental }\end{array}$ & $\begin{array}{l}\text { Categoría } \\
\text { principal }\end{array}$ & $\begin{array}{l}\text { Categoría } \\
\text { secundaria }\end{array}$ & Fuentes sonoras \\
\hline BOAPA & $\begin{array}{c}\text { Tráfico rodado } \\
\text { (valor medio de } \\
0.6 \text { y } 96 \% \text { de } \\
\text { detección de los } \\
\text { oyentes) }\end{array}$ & $\begin{array}{l}\text { Transporte } \\
\text { motorizado }\end{array}$ & $\begin{array}{c}\text { Sonidos } \\
\text { Antroposónicos }\end{array}$ & $\begin{array}{l}\text { Transporte } \\
\text { motorizado }\end{array}$ & $\begin{array}{c}\text { Avenida vehicular y } \\
\text { pájaros }\end{array}$ \\
\hline BRBRO & $\begin{array}{c}\text { Tráfico rodado } \\
\text { (valor medio de } \\
0.7 \text { y } 92 \% \text { de } \\
\text { detección de los } \\
\text { oyentes) }\end{array}$ & $\begin{array}{l}\text { Transporte } \\
\text { motorizado }\end{array}$ & $\begin{array}{c}\text { Sonidos } \\
\text { Antroposónicos }\end{array}$ & $\begin{array}{l}\text { Transporte } \\
\text { motorizado }\end{array}$ & $\begin{array}{l}\text { Avenida vehicular, } \\
\text { motocicletas, y } \\
\text { automóviles, } \\
\text { bocinas, personas } \\
\text { conversando. }\end{array}$ \\
\hline BOC197 & $\begin{array}{c}\text { Tráfico rodado } \\
\text { (valor medio de } \\
0.94 \text { y } 100 \% \text { de } \\
\text { detección de los } \\
\text { oyentes) }\end{array}$ & $\begin{array}{l}\text { Transporte } \\
\text { motorizado }\end{array}$ & $\begin{array}{c}\text { Sonidos } \\
\text { Antroposónicos }\end{array}$ & $\begin{array}{l}\text { Transporte } \\
\text { motorizado }\end{array}$ & $\begin{array}{l}\text { Avenida vehicular, } \\
\text { motocicletas, y } \\
\text { automóviles, } \\
\text { bocinas, personas } \\
\text { conversando. }\end{array}$ \\
\hline BRER & $\begin{array}{c}\text { Tráfico rodado } \\
\text { (valor medio de } \\
0.5 \text { y } 80 \% \text { de } \\
\text { detección de los } \\
\text { oyentes) }\end{array}$ & $\begin{array}{l}\text { Transporte } \\
\text { motorizado }\end{array}$ & $\begin{array}{c}\text { Sonidos } \\
\text { Antroposónicos }\end{array}$ & $\begin{array}{l}\text { Transporte } \\
\text { motorizado }\end{array}$ & $\begin{array}{c}\text { Avenida vehicular y } \\
\text { pájaros }\end{array}$ \\
\hline BRFA & $\begin{array}{c}\text { Agua (valor medio } \\
\text { de } 0.8 \text { y } 93 \% \text { de } \\
\text { detección de los } \\
\text { oyentes) }\end{array}$ & Naturaleza & $\begin{array}{c}\text { Sonidos } \\
\text { Geosónicos }\end{array}$ & Naturaleza & $\begin{array}{c}\text { Música, voces, } \\
\text { sonido de fuente de } \\
\text { agua. }\end{array}$ \\
\hline BOFAET & $\begin{array}{c}\text { Agua (valor medio } \\
\text { de } 0.8 \text { y } 100 \% \text { de } \\
\text { detección de los } \\
\text { oyentes) }\end{array}$ & Naturaleza & $\begin{array}{c}\text { Sonidos } \\
\text { Geosónicos }\end{array}$ & Naturaleza & $\begin{array}{l}\text { Fuente de agua, } \\
\text { tráfico vehicular. }\end{array}$ \\
\hline BRFTV & $\begin{array}{c}\text { Voces (valor } \\
\text { medio de } 0.8 \text { y } \\
100 \% \text { de detección } \\
\text { de los oyentes) }\end{array}$ & $\begin{array}{c}\text { Voces e } \\
\text { instrumentos } \\
\text { musicales }\end{array}$ & $\begin{array}{c}\text { Sonidos } \\
\text { Antroposónicos }\end{array}$ & $\begin{array}{c}\text { Voces e } \\
\text { instrumentos } \\
\text { musicales }\end{array}$ & $\begin{array}{c}\text { Música, } \\
\text { conversaciones, } \\
\text { sonido de dinero } \\
\text { (monedas de caen), } \\
\text { platos y cubiertos al } \\
\text { comer. }\end{array}$ \\
\hline
\end{tabular}




\begin{tabular}{|c|c|c|c|c|c|}
\cline { 2 - 6 } BOPL & $\begin{array}{c}\text { Voces (valor } \\
\text { medio de 0.5 y } \\
88 \% \text { de detección } \\
\text { de los oyentes) }\end{array}$ & $\begin{array}{c}\text { Voces e } \\
\text { instrumentos } \\
\text { musicales }\end{array}$ & $\begin{array}{c}\text { Sonidos } \\
\text { Antroposónicos }\end{array}$ & $\begin{array}{c}\text { Voces e } \\
\text { instrumentos } \\
\text { musicales }\end{array}$ & $\begin{array}{c}\text { Voces, palomas } \\
\text { volando, tráfico } \\
\text { vehicular. }\end{array}$ \\
\hline BRPOA & $\begin{array}{c}\text { Naturaleza (valor } \\
\text { medio de 0.9 y } \\
100 \% \text { de detección } \\
\text { de los oyentes) }\end{array}$ & Naturaleza & $\begin{array}{c}\text { Sonidos } \\
\text { Geosónicos }\end{array}$ & Naturaleza & $\begin{array}{c}\text { Pájaros, tráfico } \\
\text { vehicular }\end{array}$ \\
\hline \multirow{2}{*}{ BOPSB } & $\begin{array}{c}\text { Sonidos de la } \\
\text { naturaleza (valor } \\
\text { medio de 0.5 y } \\
80 \% \text { de detección } \\
\text { de los oyentes }\end{array}$ & Naturaleza & $\begin{array}{c}\text { Sonidos } \\
\text { Geosónicos }\end{array}$ & Naturaleza & $\begin{array}{c}\text { Pájaros, tráfico } \\
\text { vehicular }\end{array}$ \\
\hline
\end{tabular}

\subsubsection{Aspectos objetivos.}

\subsubsection{Parámetros binaurales y energéticos}

Las tablas 2-3 a 2-6 presentan los resultados obtenidos para las señales con ponderación A y Z de los entornos sonoros en estudio para los parámetros asociados a la IACF en su versión móvil y de largo término.

$\mathrm{Al}$ analizar los datos presentados en las tablas 2-3, se encuentra que lugares con sonidos fundamentales de fuentes de agua y voces presentaron menores valores de medidas de tendencia central para el parámetro IACC. Así mismo es interesante ver que los mayores valores para este descriptor estadístico pertenecieron a los parques BRPOA y BOPSB, esto teniendo en cuenta que según los oyentes los sonidos fundamentales de estos lugares son naturales (ver figura 2-9). Este fenómeno se presenta debido a la fuerte influencia de las fuentes sonoras de los entornos de estos parques, compuestos principalmente por avenidas de alto flujo vehicular que genera un fuerte aporte energético en bajas frecuencias y por ende altos valores de IACC. Los menores rangos y diferencias de percentiles de IACC pertenecen a las avenidas BOAPA y BRER.

Tabla 2-3. Valores de parámetros binaurales para los entornos acústicos con ponderación Z. En fondo amarillo entornos acústicos con sonidos fundamentales de tráfico vehicular, en azul sonidos fundamentales de agua y en verde sonidos fundamentales de cantos de pájaros y la naturaleza.

\begin{tabular}{|c|c|c|c|c|c|c|c|c|c|c|}
\hline $\begin{array}{c}\text { Parámetro } \\
\text { acústico }\end{array}$ & BOAPA & BRRO & BOC197 & BRER & BRFA & BRFAET & BRFTV & BOPL & BRPOA & BOPSB \\
\hline IACC $_{\text {mean }}$ & 0.89 & 0.70 & 0.80 & 0.77 & 0.53 & 0.43 & 0.29 & 0.84 & 0.91 & 0.93 \\
\hline IACC $_{\text {median }}$ & 0.89 & 0.73 & 0.81 & 0.76 & 0.54 & 0.43 & 0.29 & 0.84 & 0.93 & 0.95 \\
\hline
\end{tabular}


Segundo Capítulo. Análisis de aspectos espaciales y temporales en la evaluación de paisajes sonoros

\begin{tabular}{|c|c|c|c|c|c|c|c|c|c|c|}
\hline IACC $_{\min }$ & 0.85 & 0.47 & 0.61 & 0.71 & 0.42 & 0.35 & 0.22 & 0.77 & 0.77 & 0.75 \\
\hline IACC $_{\max }$ & 0.91 & 0.85 & 0.90 & 0.85 & 0.63 & 0.53 & 0.37 & 0.94 & 0.96 & 0.97 \\
\hline IACC $_{\text {P10 }}$ & 0.87 & 0.56 & 0.68 & 0.73 & 0.46 & 0.38 & 0.27 & 0.79 & 0.84 & 0.87 \\
\hline IACC $_{\text {P90 }}$ & 0.91 & 0.81 & 0.88 & 0.82 & 0.59 & 0.48 & 0.33 & 0.90 & 0.95 & 0.97 \\
\hline IACC $_{\text {range }}$ & 0.06 & 0.38 & 0.29 & 0.14 & 0.21 & 0.18 & 0.15 & 0.17 & 0.19 & 0.22 \\
\hline IACC $_{\text {P90-P10 }}$ & 0.04 & 0.25 & 0.20 & 0.09 & 0.13 & 0.10 & 0.06 & 0.11 & 0.11 & 0.10 \\
\hline IACC $_{\text {global }}$ & 0.89 & 0.67 & 0.79 & 0.76 & 0.52 & 0.43 & 0.27 & 0.84 & 0.92 & 0.94 \\
\hline$\tau_{\text {IACC_range }}[\mathrm{ms}]$ & 0.86 & 1.18 & 1.11 & 0.84 & 0.15 & 0.05 & 1.15 & 1.18 & 0.73 & 0.16 \\
\hline$\tau_{\text {P90-P10 }}[\mathrm{ms}]$ & 0.64 & 1.00 & 0.71 & 0.57 & 0.11 & 0.03 & 0.77 & 0.80 & 0.23 & 0.12 \\
\hline $\mathrm{W}_{\text {IACC_range }}[\mathrm{ms}]$ & 0.50 & 2.19 & 1.43 & 0.68 & 0.63 & 0.06 & 0.79 & 0.82 & 2.54 & 0.26 \\
\hline $\mathrm{W}_{\text {IACC_P90-P10 }}[\mathrm{ms}]$ & 0.30 & 1.92 & 0.914 & 0.511 & 0.32 & 0.04 & 0.46 & 0.38 & 1.00 & 0.15 \\
\hline Leq $_{\text {IdB] }}[$ & 74.4 & 79.9 & 83.8 & 73.4 & 74.5 & 77.3 & 74.6 & 80.5 & 56.7 & 71.1 \\
\hline
\end{tabular}

Tabla 2-4. Valores de parámetros binaurales para los entornos acústicos con ponderación A. En fondo amarillo entornos acústicos con sonidos fundamentales de tráfico vehicular, en azul sonidos fundamentales de agua y en verde sonidos fundamentales de cantos de pájaros y la naturaleza.

\begin{tabular}{|c|c|c|c|c|c|c|c|c|c|c|}
\hline $\begin{array}{l}\text { Parámetro } \\
\text { acústico }\end{array}$ & BOAPA & BRRO & BOC197 & BRER & BRFA & BRFAET & BRFTV & BOPL & BRPOA & BOPSB \\
\hline $\mathrm{IACC}_{\text {mean_A }}$ & 0.17 & 0.27 & 0.18 & 0.19 & 0.15 & 0.30 & 0.11 & 0.26 & 0.16 & 0.38 \\
\hline $\mathrm{IACC}_{\text {median_A }}$ & 0.16 & 0.25 & 0.18 & 0.18 & 0.15 & 0.30 & 0.10 & 0.25 & 0.14 & 0.39 \\
\hline $\mathrm{IACC}_{\text {min_A }}$ & 0.10 & 0.14 & 0.09 & 0.09 & 0.12 & 0.25 & 0.05 & 0.17 & 0.08 & 0.27 \\
\hline $\mathrm{IACC}_{\max \_\mathrm{A}}$ & 0.22 & 0.67 & 0.29 & 0.37 & 0.19 & 0.32 & 0.18 & 0.37 & 0.30 & 0.49 \\
\hline $\mathrm{IACC}_{\mathrm{P} 10 \_\mathrm{A}}$ & 0.13 & 0.16 & 0.12 & 0.11 & 0.13 & 0.28 & 0.08 & 0.21 & 0.10 & 0.32 \\
\hline $\mathrm{IACC}_{\mathrm{P} 90 \_\mathrm{A}}$ & 0.20 & 0.44 & 0.25 & 0.32 & 0.18 & 0.31 & 0.16 & 0.32 & 0.23 & 0.46 \\
\hline IACC $\mathrm{C}_{\text {range_A }}$ & 0.12 & 0.53 & 0.20 & 0.28 & 0.07 & 0.07 & 0.13 & 0.20 & 0.22 & 0.22 \\
\hline $\mathrm{IACC}_{\mathrm{P} 90-\mathrm{P} 10 \_\mathrm{A}}$ & 0.07 & 0.28 & 0.13 & 0.21 & 0.05 & 0.03 & 0.08 & 0.11 & 0.13 & 0.14 \\
\hline IACC $\mathrm{Alobal \_ A}_{\mathrm{A}}$ & 0.21 & 0.30 & 0.12 & 0.12 & 0.14 & 0.28 & 0.31 & 0.21 & 0.22 & 0.42 \\
\hline$\tau_{\mathrm{IACC} \_ \text {range_A }}[\mathrm{ms}]$ & 1.27 & 1.75 & 1.55 & 1.02 & 0.18 & 0.03 & 1.72 & 1.68 & 1.57 & 0.07 \\
\hline$\tau_{\text {IACC_P90-P10_A }}[\mathrm{ms}]$ & 1.13 & 1.32 & 0.88 & 0.84 & 0.13 & 0.03 & 1.23 & 1.34 & 0.61 & 0.05 \\
\hline $\mathrm{W}_{\mathrm{IACC} \_ \text {range_A }}[\mathrm{ms}]$ & 0.23 & 5.39 & 0.37 & 0.21 & 0.11 & 0.02 & 0.33 & 0.5 & 0.42 & 0.2 \\
\hline WIACC_P90-P10_A $[\mathrm{ms}]$ & 0.08 & 0.20 & 0.14 & 0.1 & 0.07 & 0.01 & 0.13 & 0.30 & 0.111 & 0.11 \\
\hline LAeq $[\mathrm{dB}]$ & 63.9 & 71.5 & 74.6 & 64.7 & 71.3 & 75.4 & 71.8 & 68.7 & 45.6 & 51.4 \\
\hline
\end{tabular}




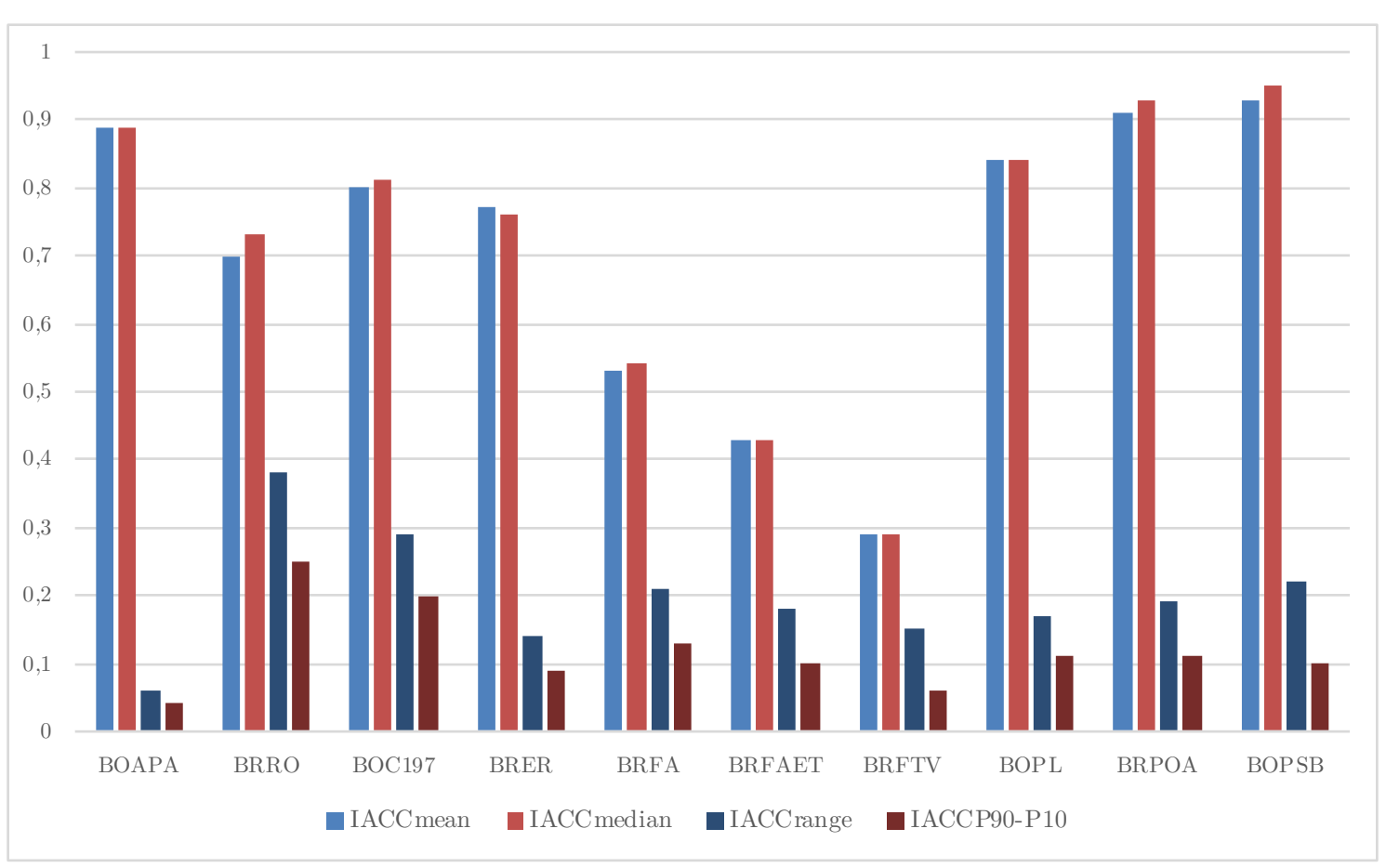

Figura 2-9. Descriptores estadísticos para el parámetro IACC en señales con ponderación Z.

A fin de determinar diferencias estadísticamente significativas en los diferentes descriptores estadísticos de IACC según la fuente generadora del sonido fundamental, fueron aplicadas las pruebas de Kruskal-Wallis con un $\alpha=0.05$. Según los resultados obtenidos (tabla 2-5) estas diferencias no fueron encontradas (tabla 2-5).

Tabla 2-5. Resultados de las pruebas de Kruskal-Wallis con $\alpha=0.05$ para señales con ponderación $Z$ según las categorías de sonidos fundamentales y parámetros binaurales. Valores sombreados en gris representan diferencias estadísticamente significativas entre el parámetro acústico y la fuente sonora generadora del sonido fundamental.

\begin{tabular}{|l|l|l|l|l|l|l|l|l|l|l|l|l|l|l|}
\hline & IACC $_{\text {mean }}$ & IACC $_{\text {median }}$ & $\begin{array}{l}\text { IACC } \\
\text { min }\end{array}$ & $\begin{array}{l}\text { IACC } \\
\text { max }\end{array}$ & $\begin{array}{l}\text { IACC } \\
\text { P10 }\end{array}$ & $\begin{array}{l}\text { IACC } \\
\text { P90 }\end{array}$ & $\begin{array}{l}\text { IACC } \\
\text { range }\end{array}$ & $\begin{array}{l}\text { IACC } \\
\text { P90-P10 }\end{array}$ & $\begin{array}{l}\tau_{\text {IACC }} \\
\text { range }\end{array}$ & $\begin{array}{l}\tau_{\text {IACC }} \\
\text { P90-P10 }\end{array}$ & $\tau_{\text {IACCrange }}$ & $\begin{array}{l}\text { W }_{\text {IACC }} \\
\text { P90-P10 }\end{array}$ & $\begin{array}{l}\text { IACC } \\
\text { global }\end{array}$ & Leq \\
\hline $\begin{array}{l}\text { Asymp. } \\
\text { sig. }\end{array}$ & 0.73 & 0.73 & 0.80 & 0.84 & 0.71 & 0.73 & 0.52 & 0.80 & $\mathbf{0 . 0 2}$ & $\mathbf{0 . 0 3}$ & 0.56 & 0.37 & 0.73 & 0.232 \\
\hline
\end{tabular}

$\mathrm{Al}$ analizar los resultados de IACC para las señales con ponderación A (Tabla 2-4), puede verse que los valores de este parámetro disminuyeron notablemente para todos los descriptores estadísticos (Figura 2-10). Sin embargo, se siguen presentando comportamientos similares a los resultados obtenidos con las señales con ponderación Z, donde lugares con sonidos fundamentales de voces presentaron menores valores de medidas de tendencia central para el parámetro IACC. Así mismo, al aplicar las pruebas de Kruskal-Wallis tampoco fueron 
encontradas diferencias estadísticamente significativas según la fuente del sonido fundamental para ninguno de los estadísticos descriptivos usados (tabla 2-6).

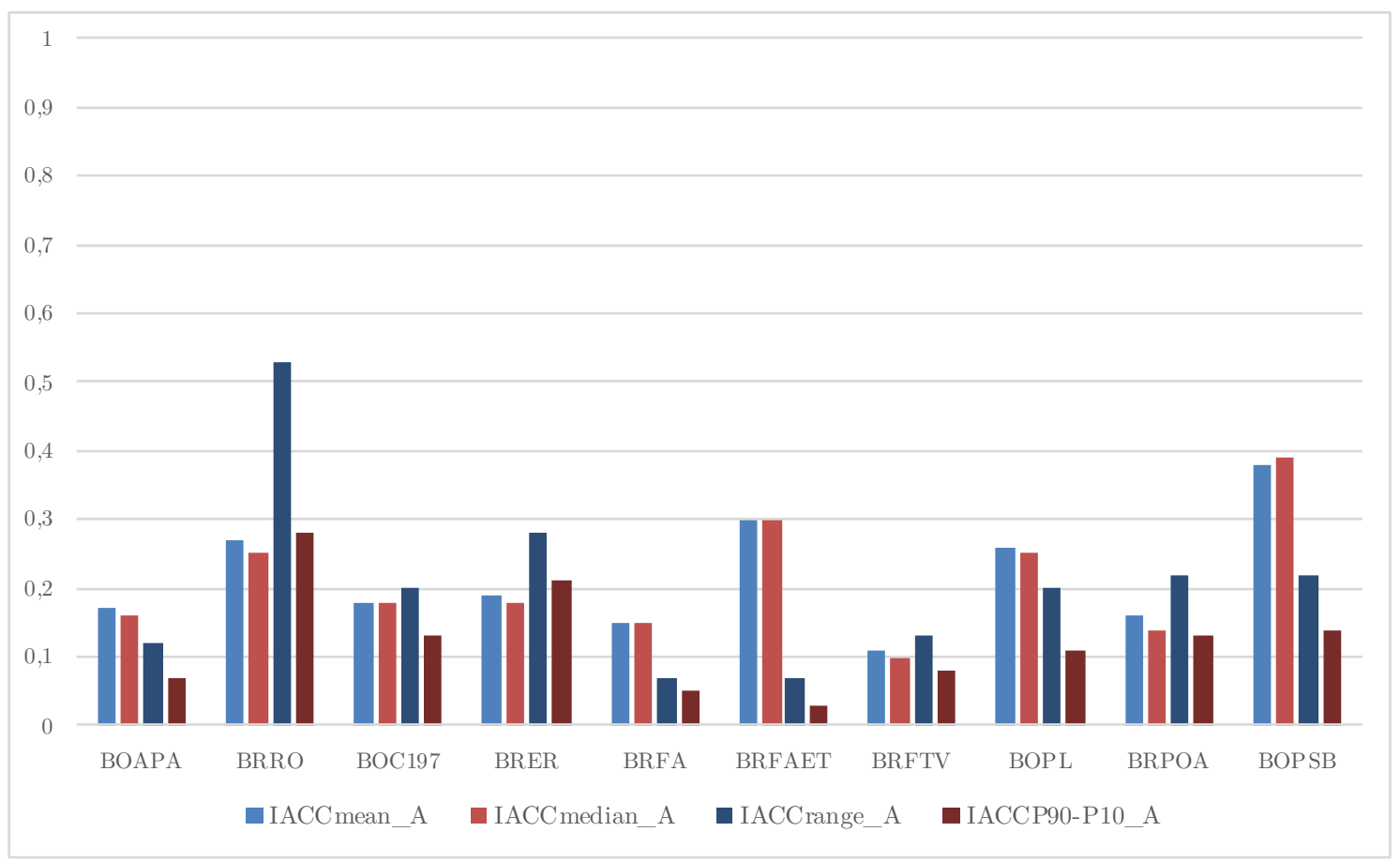

Figura 2-10. Descriptores estadísticos para el parámetro IACC en señales con ponderación Z.

Tabla 2-6. Resultados de las pruebas de Kruskal-Wallis con $\alpha=0.05$ para señales con ponderación A según las categorías de sonidos fundamentales y parámetros binaurales. Valores sombreados en gris representan diferencias estadísticamente significativas entre el parámetro acústico y la fuente sonora generadora del sonido fundamental.

\begin{tabular}{|l|l|l|l|l|l|l|l|l|l|l|l|l|l|l|}
\hline & $\begin{array}{l}\text { IACC } \\
\text { meanA }\end{array}$ & $\begin{array}{l}\text { IACC } \\
\text { median A }\end{array}$ & $\begin{array}{l}\text { IACC } \\
\text { minA }\end{array}$ & $\begin{array}{l}\text { IACC } \\
\text { maxA }\end{array}$ & $\begin{array}{l}\text { IACC } \\
\text { P10A }\end{array}$ & $\begin{array}{l}\text { IACC } \\
\text { P90A }\end{array}$ & $\begin{array}{l}\text { IACC } \\
\text { rangeA }\end{array}$ & $\begin{array}{l}\text { IACC } \\
\text { P90-P10A }\end{array}$ & $\begin{array}{l}\tau_{\text {IACC }} \\
\text { rangeA }\end{array}$ & $\begin{array}{l}\tau_{\text {IACC }} \\
\text { P90-P10A }\end{array}$ & $\begin{array}{l}\text { wIACC } \\
\text { rangeA }\end{array}$ & $\begin{array}{l}\text { WIACC } \\
\text { P90-P10A }\end{array}$ & $\begin{array}{l}\text { IACC } \\
\text { Global_A }\end{array}$ & LAeq \\
\hline $\begin{array}{l}\text { Asymp. } \\
\text { sig. }\end{array}$ & 0.73 & 0.79 & 0.56 & 0.77 & 0.62 & 0.77 & 0.49 & 0.38 & 0.11 & $\mathbf{0 . 0 2}$ & 0.21 & 0.14 & 0.37 & 0.782 \\
\hline
\end{tabular}

Para el parámetro $\tau_{\text {IACC }}$ y sus estadísticos descriptivos se encontró que, para señales con y sin ponderación A, los menores valores correspondían a lugares con sonidos fundamentales naturales (tablas 2-3 y 2-4). De forma específica, los menores valores de rangos y diferencias de percentiles 90-10 correspondían a las fuentes de agua, lo que era lógico pues estos entornos acústicos presentan una fuente acústica dominante (la fuente de agua), teniendo una clara ubicación en el espacio (figura 2-11). Por otra parte, los lugares con mayores rangos de $\tau_{\text {IACC }}$ correspondieron a entornos con sonidos fundamentales de voces y centros urbanos (BRRO y BOC197). 


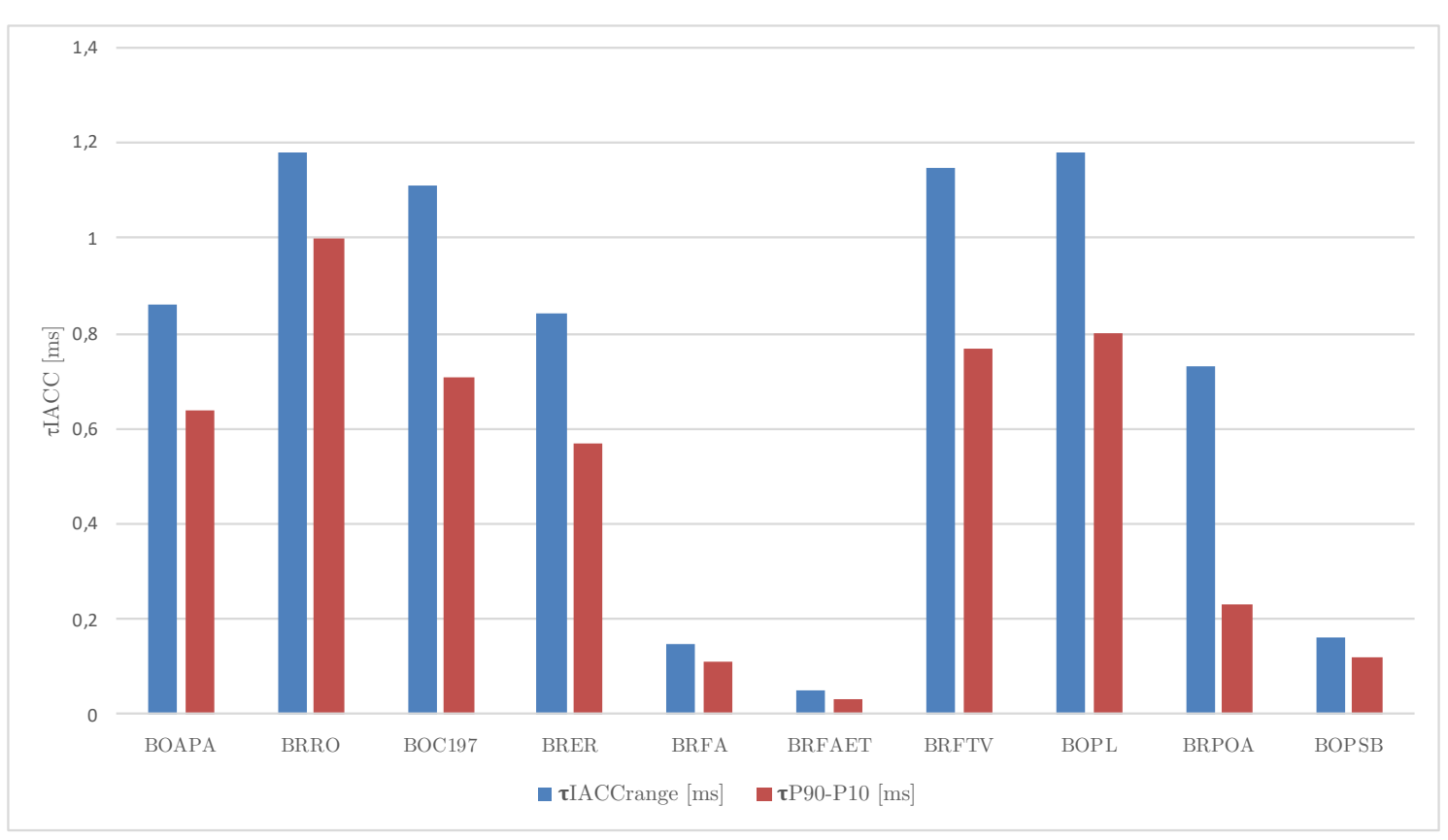

Figura 2-11. Descriptores estadísticos para el parámetro $\boldsymbol{\tau}_{I A C C}$ en señales con ponderación $Z$.

En las señales con ponderación A (figura 2-12) se pueden apreciar mayores rangos y diferencias de percentiles de $\tau_{\text {IACC }}$ respecto a señales con ponderación $\mathrm{Z}$, lo que representa el indudable aporte energético de las frecuencias bajas en aspectos de ubicaciones espaciales de fuentes sonoras. De nuevo centros de ciudades y lugares con sonidos fundamentales de voces presentan los mayores rangos y diferencias percentiles.

Al aplicar las pruebas de Kruskal-Wallis en el parámetro $\tau_{\mathrm{IACC}}$ en señales con ponderación $\mathrm{Z}$, fueron detectadas diferencias estadísticamente significativas según la fuente del sonido fundamental, tanto para los rangos como para las diferencias de percentiles (tabla 2-5). En las señales con ponderación A también fueron encontradas estas diferencias estadísticamente significativas, aunque en este caso solo bajo el descriptor de diferencia de percentiles 90-10 (tabla 2-6). 


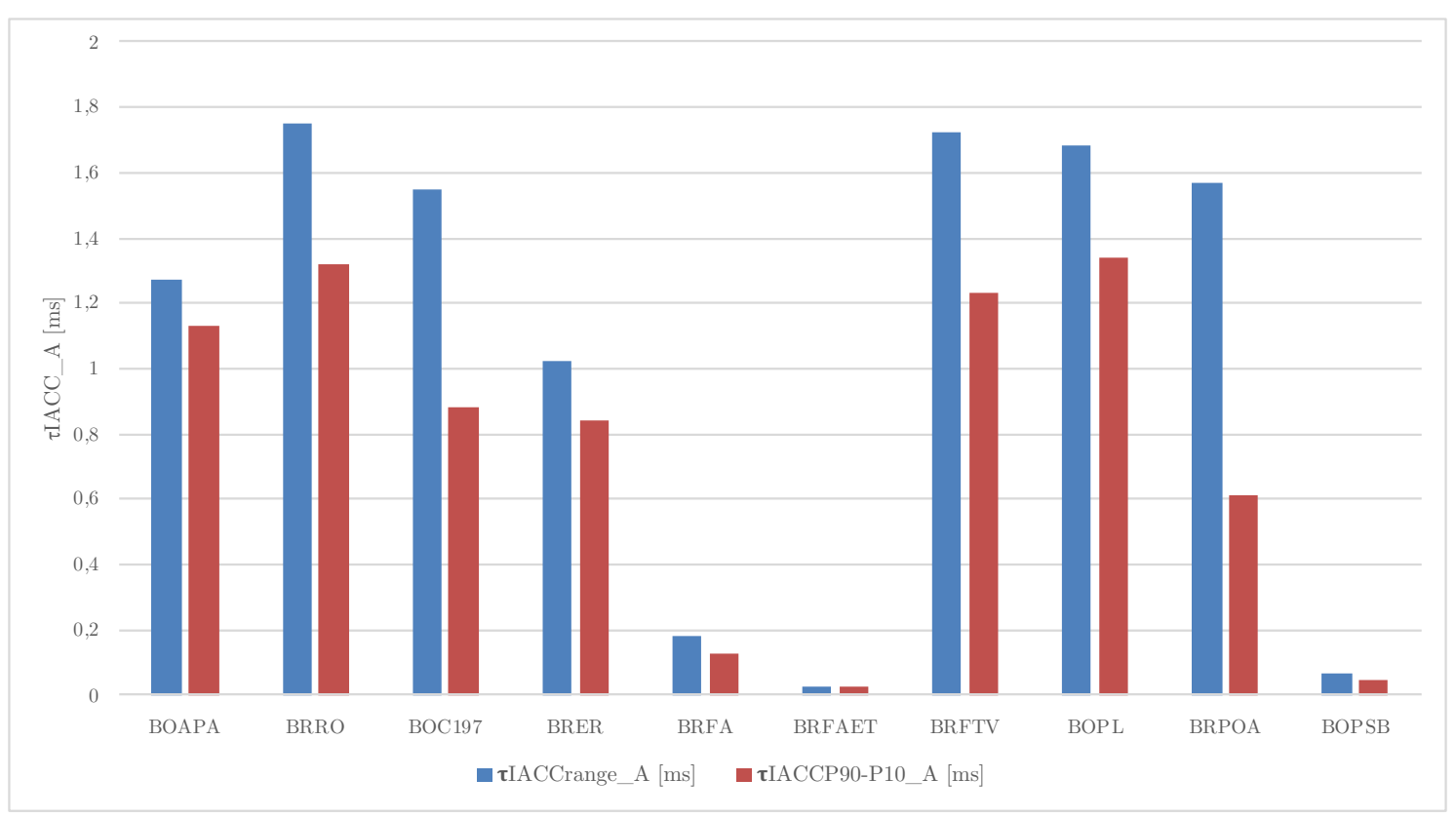

Figura 2-12. Descriptores estadísticos para el parámetro $\boldsymbol{\tau}_{I A C C}$ en señales con ponderación $A$.

Los resultados de rangos de WIACC para las señales ponderadas y no ponderadas muestran que lugares con una única fuente dominante (fuentes de agua o grandes avenidas) presentaron los menores rangos, mientras que entornos con sonidos fundamentales de voces presentaron grandes rangos y diferencias de percentiles, lo que indica variabilidad espacial en los entornos acústicos, aunque no fueron encontradas diferencias estadísticamente significativas según su sonido fundamental (tablas 2-5 y 2-6).

Respecto a los resultados obtenidos por el descriptor energético, el nivel continuo equivalente Leq, los niveles más bajos corresponden a parques con sonidos fundamentales naturales, mientras que los mayores niveles corresponden a lugares con sonidos fundamentales antroposónicos (Tabla 2-3). Para las señales con ponderación A, los niveles más bajos se asociaron nuevamente con sonidos fundamentales naturales, mientras que los mayores niveles se presentaron en los lugares con fuentes de agua, tráfico rodado y voces. Así mismo, los resultados permiten apreciar que lugares con fuentes de agua presentaron las menores variaciones entre los niveles Leq y LeqA, lo que puede atribuirse al espectro amplio en frecuencia que presentan este tipo de fuentes sonoras. No se presentan diferencias estadísticamente significativas según la fuente sonora del sonido fundamental y el Leq, para las señales con ponderación A y Z. 


\subsubsection{Parámetros temporales}

Las tablas 2-7 a 2-10 presentan los resultados obtenidos para las señales con ponderación A y Z de los entornos sonoros en estudio para los parámetros asociados a la ACF en su versión móvil y de largo término.

Tabla 2-7. Valores de parámetros temporales para los entornos acústicos con ponderación Z. En fondo amarillo entornos acústicos con sonidos fundamentales de tráfico vehicular, en azul sonidos fundamentales de agua y en verde sonidos fundamentales de cantos de pájaros y la naturaleza.

\begin{tabular}{|c|c|c|c|c|c|c|c|c|c|c|}
\hline $\begin{array}{c}\text { Parámetro } \\
\text { acústico } \\
{[\mathrm{ms}]}\end{array}$ & BOAPA & BRRO & BOC197 & BRER & BRFA & BRFAET & BRFTV & BOPL & BRPOA & BOPSB \\
\hline$\tau_{\mathrm{e}}$ mean & 6.22 & 5.99 & 6.46 & 10.18 & 4.14 & 3.20 & 2.97 & 10.39 & 9.47 & 10.05 \\
\hline$\tau_{\mathrm{e}}$ median & 5.75 & 5.51 & 6.25 & 9.91 & 3.75 & 2.19 & 2.58 & 9.64 & 7.90 & 10.99 \\
\hline$\tau_{\mathrm{e}} \min$ & 4.60 & 3.99 & 4.15 & 3.36 & 1.96 & 1.08 & 1.10 & 3.38 & 6.06 & 5.18 \\
\hline$\tau_{\mathrm{e}} \max$ & 20.30 & 14.58 & 19.04 & 36.94 & 10.19 & 18.90 & 8.10 & 45.51 & 21.96 & 18.42 \\
\hline$\tau_{\mathrm{e}}$ P10 & 5.01 & 4.55 & 4.67 & 4.97 & 2.40 & 1.89 & 1.79 & 4.31 & 6.59 & 5.89 \\
\hline$\tau_{\mathrm{e}}$ P90 & 7.18 & 8.02 & 8.17 & 14.21 & 6.64 & 3.14 & 5.34 & 14.12 & 14.88 & 13.56 \\
\hline$\tau_{\mathrm{e}}$ range & 15.70 & 10.59 & 14.89 & 33.58 & 8.23 & 17.82 & 7.00 & 42.13 & 15.90 & 13.24 \\
\hline$\tau_{\mathrm{e}}$ P90-P10 & 2.17 & 3.47 & 3.50 & 9.24 & 4.24 & 1.25 & 3.55 & 9.81 & 8.29 & 7.68 \\
\hline$\tau_{\mathrm{e}}$ Global & 85.00 & 12.60 & 22.00 & 20.8 & 6.90 & 2.50 & 0.78 & 14.8 & 141.9 & 56.70 \\
\hline
\end{tabular}

Tabla 2-8. Valores de parámetros temporales para los entornos acústicos con ponderación A. En fondo amarillo entornos acústicos con sonidos fundamentales de tráfico vehicular, en azul sonidos fundamentales de agua y en verde sonidos fundamentales de cantos de pájaros y la naturaleza.

\begin{tabular}{|c|c|c|c|c|c|c|c|c|c|c|}
\hline $\begin{array}{c}\text { Parámetro } \\
\text { acústico } \\
{[\mathrm{ms}]}\end{array}$ & BOAPA & BRRO & BOC197 & BRER & BRFA & BRFAET & BRFTV & BOPL & BRPOA & BOPSB \\
\hline$\tau_{\text {e mean_A }}$ & 1.14 & 2.85 & 3.23 & 1.03 & 0.94 & 1.02 & 2.43 & 2.77 & 1.32 & 3.23 \\
\hline$\tau_{\text {e median_A }}$ & 1.14 & 1.56 & 1.36 & 1.00 & 0.99 & 1.02 & 1.66 & 1.72 & 1.25 & 2.13 \\
\hline$\tau_{\text {e min A }}$ & 0.83 & 0.77 & 0.65 & 0.78 & 0.65 & 0.68 & 1.01 & 0.84 & 0.72 & 1.38 \\
\hline$\tau_{\text {e max A }}$ & 2.68 & 31.18 & 42.20 & 1.44 & 1.16 & 1.21 & 15.53 & 15.08 & 5.90 & 8.82 \\
\hline$\tau_{\text {e P10 A }}$ & 1.01 & 1.02 & 0.95 & 0.85 & 0.70 & 0.91 & 1.37 & 0.95 & 0.98 & 1.72 \\
\hline$\tau_{\text {e P90 A }}$ & 1.29 & 8.21 & 3.02 & 1.18 & 1.06 & 1.12 & 4.40 & 4.44 & 1.71 & 6.98 \\
\hline$\tau_{\text {e range A }}$ & 1.85 & 30.41 & 41.55 & 0.66 & 0.51 & 0.53 & 14.52 & 14.24 & 5.18 & 7.44 \\
\hline$\tau_{\text {e P90-P10_A }}$ & 0.28 & 7.20 & 2.07 & 0.33 & 0.36 & 0.21 & 3.03 & 3.49 & 0.73 & 5.26 \\
\hline
\end{tabular}

Los datos presentados en la tabla 2-7 y la figura 2-13 permiten apreciar que los menores valores de $\tau_{\mathrm{e}}$ para sus diferentes descriptores estadísticos provenían de ambientes sonoros con sonidos fundamentales de agua y voz. Los entornos acústicos que presentaron mayores valores de $\tau_{\mathrm{e}}$ tenían como sonido fundamental el tráfico vehicular. No fueron encontradas diferencias estadísticamente significativas en $\tau_{\mathrm{e}}$ para ningún estadístico descriptivo según las 
categorías de fuente de sonido fundamental bajo ningún nivel de significancia (tabla 2-9).

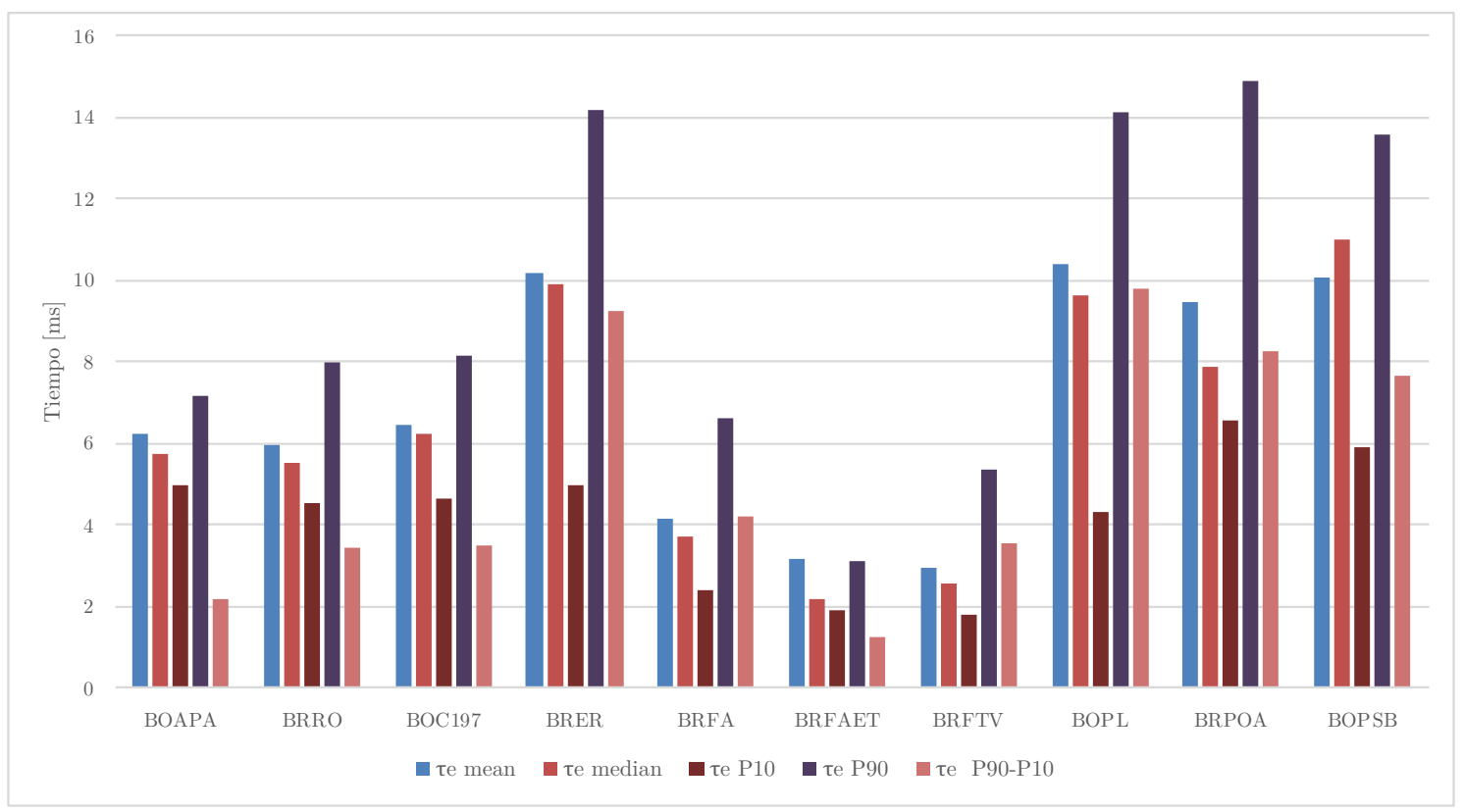

Figura 2-13. Descriptores estadísticos para el parámetro $\tau_{e}$ en señales con ponderación $Z$.

Para las señales ponderadas A pueden apreciarse valores mucho menores del parámetro $\tau_{\mathrm{e}}$, lo que se atribuye a que al aporte energético que realizan las frecuencias bajas en las señales con ponderación Z. Así mismo, en todos los descriptores estadísticos $\tau_{\mathrm{e}}$ se encontró que los valores más bajos correspondieron a entornos con sonidos fundamentales de agua (tabla 2-8 y figura 2-14). Quienes presentaban los valores más altos de $\tau_{\mathrm{e}}$ correspondían a entornos con sonidos fundamentales de tráfico vehicular y voces, siendo los de tráfico vehicular quienes presentaban los mayores rangos. Sin embargo, la tabla 2-10 muestra que no existen diferencias estadísticamente significativas según la fuente del sonido fundamental para ningún descriptor estadístico de $\tau_{\mathrm{e}}$ bajo ningún nivel de significancia. 


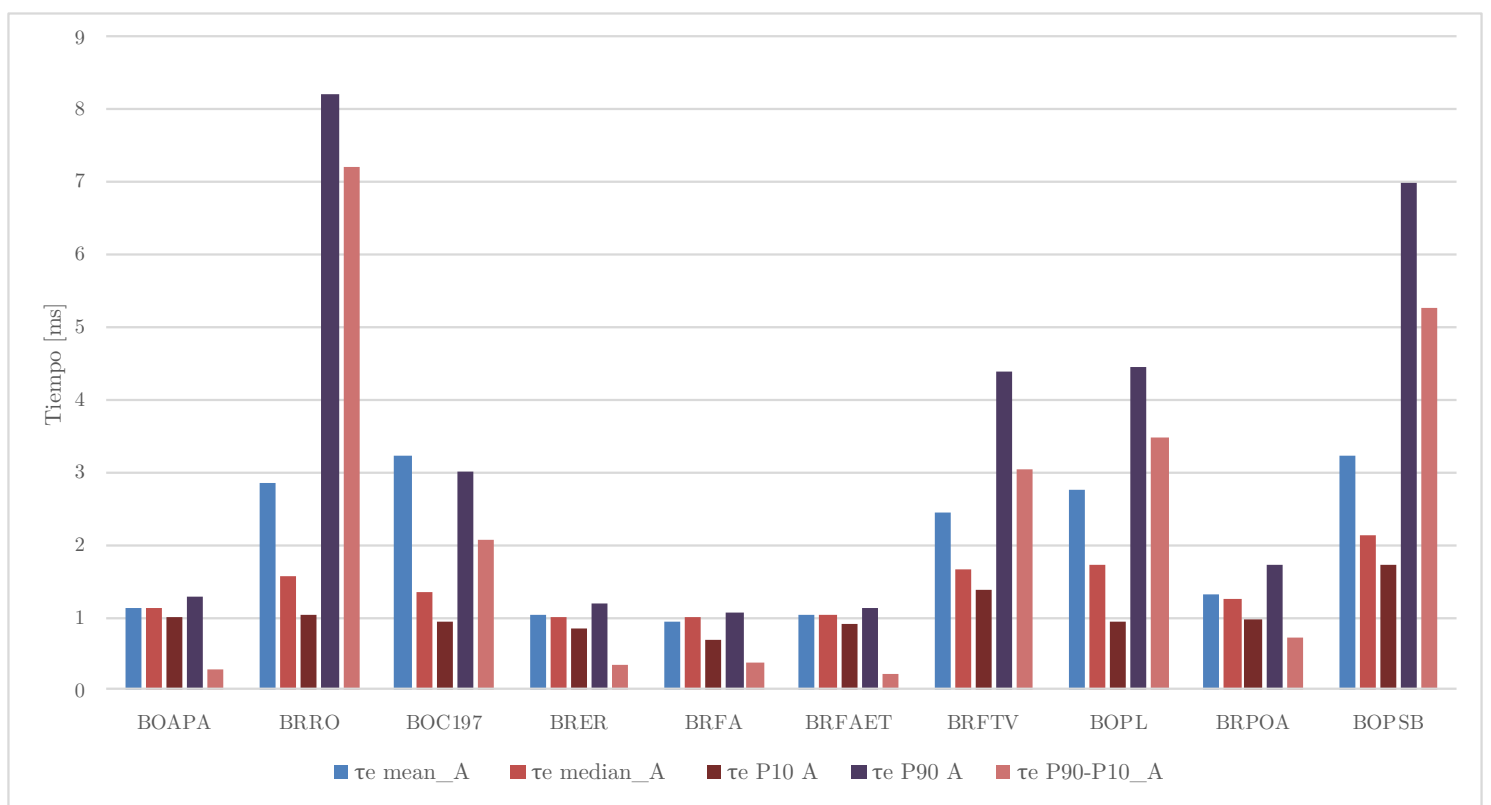

Figura 2-14. Descriptores estadísticos para el parámetro $\tau_{e}$ en señales con ponderación A.

Tabla 2-9. Resultados de las pruebas de Kruskal-Wallis con $\alpha=0.05$ para señales con ponderación $Z$ según las categorías de sonidos fundamentales y parámetros temporales.

\begin{tabular}{|c|c|c|c|c|c|c|c|c|c|}
\hline & $\tau_{\mathrm{e}}$ mean & $\tau_{\mathrm{e}}$ median & $\tau_{\mathrm{e}} \min$ & $\tau_{\mathrm{e}} \max$ & $\tau_{\mathrm{e}} \mathrm{P} 10$ & $\tau_{\mathrm{e}}$ P90 & $\tau_{\mathrm{e}}$ range & $\tau_{\mathrm{e}}$ P90-P10 & $\tau_{\mathrm{e}}$ global \\
\hline $\begin{array}{c}\text { Sig. } \\
\text { asintót. }\end{array}$ & 0.897 & 0.909 & 0.564 & 0.782 & 0.285 & 0.909 & 0.973 & 0.52 & 0.401 \\
\hline
\end{tabular}

Tabla 2-10. Resultados de las pruebas de Kruskal-Wallis con $\alpha=0.05$ para señales con ponderación A según las categorías de sonidos fundamentales y parámetros temporales.

\begin{tabular}{|l|c|c|c|c|c|c|c|c|}
\hline & $\tau_{\mathrm{e}}$ meanA & $\tau_{\mathrm{e}}$ medianA & $\tau_{\mathrm{e}} \operatorname{minA}$ & $\tau_{\mathrm{e}} \operatorname{maxA}$ & $\tau_{\mathrm{e}} \mathrm{P} 10 \mathrm{~A}$ & $\tau_{\mathrm{e}} \mathrm{P} 90 \mathrm{~A}$ & $\tau_{\mathrm{e}}$ rangeA & $\tau_{\mathrm{e}} \mathrm{P} 90-\mathrm{P} 10 \mathrm{~A}$ \\
\hline Sig. asintót. & 0.623 & 0.293 & 0.289 & 0.217 & 0.795 & 0.453 & 0.217 & 0.564 \\
\hline
\end{tabular}

2.3.3 Aspectos subjetivos y estudios de correlación entre parámetros objetivos y atributos perceptuales.

La tabla 2-11 presenta los valores medios para cada uno de los ocho atributos perceptuales del modelo de Axelsson, así como la media de la evaluación general del entorno acústico. La figura 2-15 presenta la representación gráfica de los resultados provenientes de los componentes principales en el sistema de coordenadas cartesianas según el modelo de Axelsson. 
Segundo Capítulo. Análisis de aspectos espaciales y temporales en la evaluación de paisajes sonoros

Tabla 2-11. Valores medios de parámetros subjetivos.

\begin{tabular}{|c|c|c|c|c|c|c|c|c|c|}
\hline Lugar & $\begin{array}{c}\text { Evaluación } \\
\text { general }\end{array}$ & Agradable & Caótico & $\begin{array}{c}\text { Excitante } \\
\text { /Vibrante }\end{array}$ & $\begin{array}{c}\text { Uniforme/ } \\
\text { sin eventos }\end{array}$ & Calmo & Desagradable & Agitado & Monótono \\
\hline BRAPA & -0.06 & -0.30 & 0.06 & -0.30 & 0.30 & -0.40 & 0.08 & -0.06 & 0.30 \\
\hline BRRO & -0.18 & -0.38 & 0.16 & -0.18 & -0.36 & -0.64 & 0.26 & 0.52 & -0.18 \\
\hline BOC197 & -0.62 & -0.82 & 0.62 & -0.28 & -0.54 & -0.94 & 0.50 & 0.42 & -0.38 \\
\hline BRER & 0.24 & 0.08 & -0.28 & -0.10 & 0.18 & -0.18 & -0.22 & -0.26 & 0.44 \\
\hline BOFA & 0.36 & 0.18 & -0.42 & -0.10 & 0.30 & 0.04 & -0.44 & -0.26 & 0.22 \\
\hline BRFAET & 0.38 & 0.22 & -0.30 & -0.30 & 0.50 & 0.18 & -0.58 & -0.36 & 0.30 \\
\hline BRFTV & -0.06 & -0.24 & 0.02 & -0.30 & 0.32 & -0.68 & 0.04 & 0.34 & -0.12 \\
\hline BOPL & -0.26 & -0.42 & 0.38 & -0.38 & -0.48 & -0.74 & 0.28 & 0.46 & -0.28 \\
\hline BRPOA & 0.90 & 0.98 & -0.96 & 0.28 & 0.06 & 0.68 & -0.96 & -0.52 & -0.20 \\
\hline BOPSB & 0.42 & 0.50 & -0.68 & -0.28 & 0.42 & 0.46 & -0.60 & -0.68 & 0.28 \\
\hline
\end{tabular}

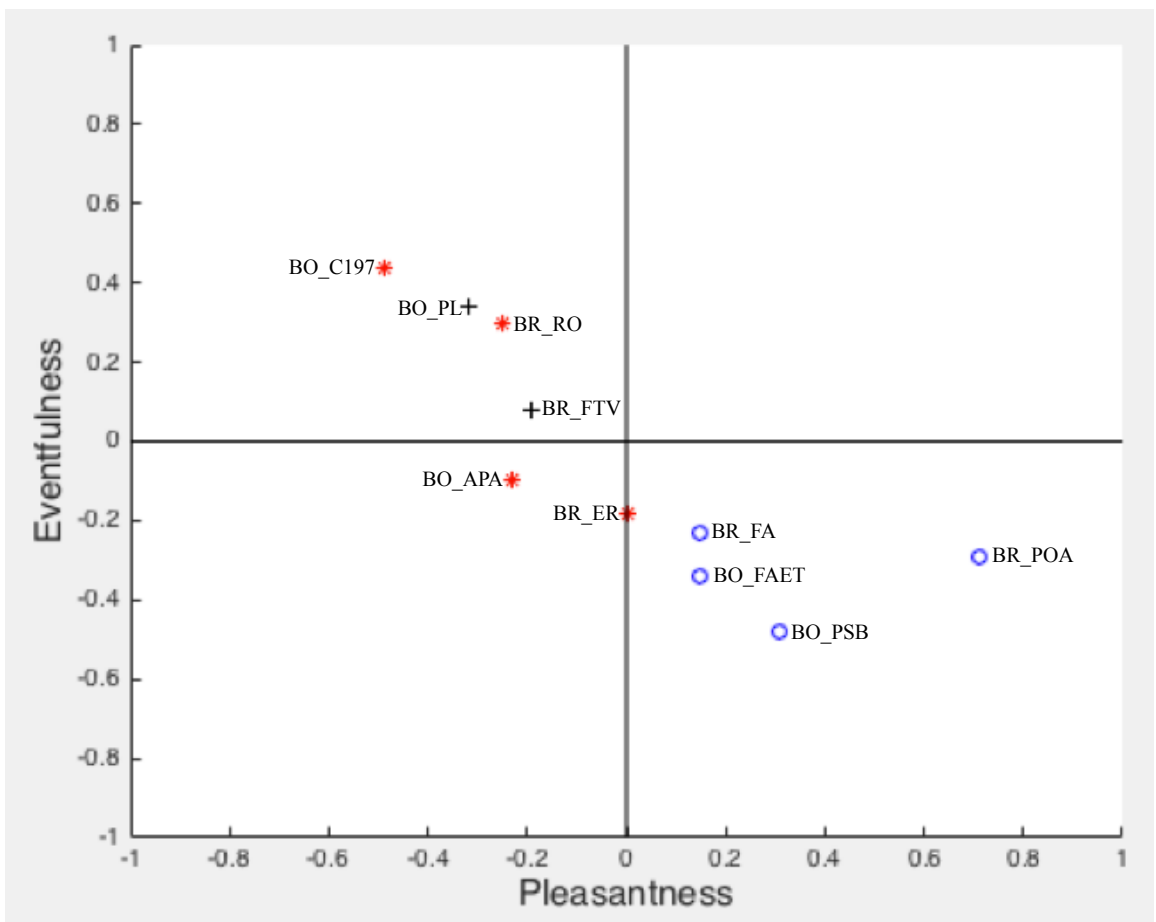

Figura 2-15. Representación gráfica de los lugares evaluados. En círculos paisajes sonoros con sonidos fundamentales naturales, en estrellas paisajes sonoros con sonidos fundamentales de tráfico rodado y en cruces paisajes sonoros con sonidos fundamentales de voces.

Se puede apreciar que lugares con sonidos fundamentales naturales (agua y pájaros) se encuentran en el cuadrante inferior derecho (placenteros y con pocos eventos). Así mismo, lugares con sonidos fundamentales de voces se encuentran en el cuadrante superior izquierdo (no placenteros y con eventos) mientras que lugares con sonidos fundamentales de tráfico vehicular se reparten entre los 
cuadrantes izquierdos superior e inferior, en cualquiera de los dos casos considerados no placenteros.

De la figura 2-15 es importante apreciar que lugares similares se encuentran en coordenadas cartesianas similares. Específicamente las fuentes de agua BOFAET y BRFA se encuentran en el cuadrante inferior derecho, parques urbanos se encuentran (BRPOA y BOPSB) se encuentran en el cuadrante inferior derecho, grandes avenidas (BRER y BRAPA) se encuentran en el cuadrante inferior izquierdo, mientras que centros de ciudad (BOC197 y BRRO) y conglomerados de personas (BOPL y BRFTV) se encuentran en el cuadrante superior izquierdo.

\subsubsection{Correlación de atributos perceptuales con parámetros binaurales derivados de la IACF.}

Pasando al estudio de correlación entre parámetros acústicos binaurales derivados de las IACF y los atributos perceptuales, las tablas 2-12 y 2-13 presentan los coeficientes de correlación de Spearman tanto para las señales con ponderación Z como con ponderación A.

La tabla 2-12 presenta una correlación negativa moderada para el parámetro IACC_P90-P10 con el atributo Monótono para las señales sin ponderar. El parámetro IACC se relaciona con la localización espacial en el plano horizontal de las fuentes, por lo que mientras mayor sea la variación espacial de las fuentes sonoras en el espacio, más grandes serán las diferencias de los percentiles de IACC. En este caso, al existir una correlación negativa moderada entre la diferencia de percentiles de IACC y el parámetro Monótono, se puede inferir que cuanto mayor sean estas diferencias (mayores variaciones de la localización espacial) menos monótono es percibido el lugar. Respecto a los parámetros con ponderación A no se encuentra ningún tipo de correlación bajo ningún nivel de significancia (tabla $2-13)$. 
Segundo Capítulo. Análisis de aspectos espaciales y temporales en la evaluación de paisajes sonoros

Tabla 2-12. Coeficientes de correlación de Spearman entre atributos perceptuales, parámetros binaurales y Leq con ponderación Z.

\begin{tabular}{|c|c|c|c|c|c|c|c|c|c|c|c|}
\hline & $\begin{array}{c}\text { Evaluación } \\
\text { general }\end{array}$ & Agradable & Caótico & $\begin{array}{l}\text { Excitante } \\
\text { /Vibrante } \\
\end{array}$ & $\begin{array}{c}\text { Uniforme/sin } \\
\text { eventos }\end{array}$ & Calmo & Desagradable & Agitado & Monótono & Pleasantness & Evenfulness \\
\hline $\begin{array}{c}\text { IACC } \\
\text { mean } \\
\end{array}$ & 0.207 & 0.164 & -0.176 & 0.142 & -0.243 & 0.285 & -0.164 & -0.322 & -0.14 & 0.17 & -0.176 \\
\hline $\begin{array}{c}\text { IACC } \\
\text { median } \\
\end{array}$ & 0.207 & 0.164 & -0.176 & 0.142 & -0.243 & 0.285 & -0.164 & -0.322 & -0.14 & 0.17 & -0.176 \\
\hline $\mathrm{IACC}_{\min }$ & 0.024 & -0.03 & 0.018 & 0.006 & -0.354 & 0.116 & 0.03 & -0.104 & -0.061 & -0.024 & 0.018 \\
\hline $\mathrm{IACC}_{\max }$ & 0.159 & 0.122 & -0.134 & 0.108 & -0.311 & 0.237 & -0.122 & -0.253 & -0.256 & 0.128 & -0.122 \\
\hline $\mathrm{IACC}_{\mathrm{P} 10}$ & 0.174 & 0.122 & -0.134 & 0.077 & -0.18 & 0.261 & -0.122 & -0.287 & 0.046 & 0.128 & -0.17 \\
\hline $\mathrm{IACC}_{\mathrm{P} 90}$ & 0.207 & 0.164 & -0.176 & 0.142 & -0.243 & 0.285 & -0.164 & -0.322 & -0.14 & 0.17 & -0.176 \\
\hline $\begin{array}{c}\mathrm{IACC}_{\mathrm{r}} \\
\text { ange }\end{array}$ & -0.055 & -0.042 & 0.018 & 0.352 & -0.286 & 0.018 & 0.042 & 0.097 & -0.529 & -0.03 & 0.055 \\
\hline $\begin{array}{l}\text { IACC } \\
\text { P90-P10 }\end{array}$ & -0.263 & -0.256 & 0.213 & 0.363 & -0.606 & -0.171 & 0.256 & 0.349 & $-.670\left(^{*}\right)$ & -0.235 & 0.317 \\
\hline$\tau_{\text {IACCrange }}$ & $-.805(* *)$ & $-.790(* *)$ & $.778(* *)$ & -0.334 & $-.659\left(^{*}\right)$ & $-.796(* *)$ & $\left..790{ }^{* *}\right)$ & $.872(* *)$ & -0.537 & $-.787(* *)$ & $.863\left(^{* *}\right)$ \\
\hline WIACCrange $_{\text {I }}$ & -0.353 & -0.333 & 0.297 & 0.358 & $-.815(* *)$ & -0.333 & 0.333 & 0.486 & $-.760\left(^{*}\right)$ & -0.316 & 0.576 \\
\hline $\begin{array}{l}\text { WIACC } \\
\text { P90-P10 } \\
\end{array}$ & -0.34 & -0.321 & 0.285 & 0.506 & $-.754\left(^{*}\right)$ & -0.309 & 0.321 & 0.474 & -0.553 & -0.304 & 0.539 \\
\hline $\begin{array}{c}\text { IACC } \\
\text { global } \\
\end{array}$ & 0.207 & 0.164 & -0.176 & 0.142 & -0.243 & 0.285 & -0.164 & -0.322 & -0.14 & 0.17 & -0.176 \\
\hline Leq & $-0.863(* *)$ & $-0.867(* *)$ & $.903(* *)$ & -0.593 & -0.468 & $-0.818(* *)$ & $0.867(* *)$ & $.802(* *)$ & -0.438 & $-0.888(* *)$ & $0.770(* *)$ \\
\hline
\end{tabular}

* Correlación significativa al nivel de 0.05 (bilateral)

** Correlación significativa al nivel de 0.01 (bilateral) 
Segundo Capítulo. Análisis de aspectos espaciales y temporales en la evaluación de paisajes sonoros

Tabla 2-13. Coeficientes de correlación de Spearman entre atributos perceptuales y parámetros binaurales con ponderación A.

\begin{tabular}{|c|c|c|c|c|c|c|c|c|c|c|c|}
\hline & $\begin{array}{c}\text { Evaluación } \\
\text { general }\end{array}$ & Agradable & Caótico & $\begin{array}{l}\text { Excitante } \\
\text { /Vibrante }\end{array}$ & $\begin{array}{l}\text { Uniforme/ } \\
\text { sin eventos }\end{array}$ & Calmo & Desagradable & Agitado & Monótono & Pleasantness & Evenfulness \\
\hline $\mathrm{IACC}_{\text {mean_A }}$ & 0.061 & 0.042 & 0.042 & -0.21 & 0.116 & 0.164 & -0.042 & -0.116 & 0.182 & 0 & -0.236 \\
\hline $\begin{array}{c}\text { IACC } \\
\text { median_A }\end{array}$ & -0.012 & -0.03 & 0.104 & -0.286 & 0.116 & 0.085 & 0.03 & -0.07 & 0.153 & -0.067 & -0.189 \\
\hline $\mathrm{IACC}_{\text {min_A }}$ & 0.122 & 0.097 & -0.061 & -0.316 & 0.28 & 0.219 & -0.097 & -0.143 & 0.195 & 0.079 & -0.328 \\
\hline IACC max_A $_{\perp}$ & 0.03 & 0.018 & 0.03 & 0.09 & -0.177 & 0.152 & -0.018 & 0.021 & 0.037 & -0.006 & -0.103 \\
\hline IACC ${ }_{\mathrm{P} 10 \_\mathrm{A}}$ & 0.076 & 0.049 & -0.006 & -0.368 & 0.256 & 0.176 & -0.049 & -0.113 & 0.155 & 0.027 & -0.286 \\
\hline $\mathrm{IACC}_{\mathrm{P} 90 \_\mathrm{A}}$ & -0.006 & -0.018 & 0.067 & 0.015 & -0.14 & 0.103 & 0.018 & -0.015 & 0.049 & -0.043 & -0.103 \\
\hline $\begin{array}{l}\text { IACC } \\
\text { range_A } \\
\end{array}$ & -0.067 & -0.061 & 0.061 & 0.427 & -0.457 & 0 & 0.061 & 0.138 & -0.169 & -0.061 & 0.147 \\
\hline $\begin{array}{c}\text { IACC } \\
\text { P90-P10_A }\end{array}$ & -0.14 & -0.134 & 0.122 & 0.43 & -0.47 & -0.073 & 0.134 & 0.171 & -0.177 & -0.128 & 0.195 \\
\hline$\tau_{\text {IACC range } \_A}$ & -0.565 & -0.539 & 0.515 & -0.074 & -0.614 & -0.576 & 0.539 & $.742(*)$ & -0.626 & -0.529 & $.721\left(^{*}\right)$ \\
\hline $\begin{array}{c}\tau_{\mathrm{IACC}} \\
\mathrm{P} 90-\mathrm{P} 10 \_\mathrm{A} \\
\end{array}$ & $-.790(* *)$ & $-.782(* *)$ & $.758\left(^{*}\right)$ & -0.364 & -0.626 & $-.782(* *)$ & $.782\left(^{* *}\right)$ & $.881(* *)$ & -0.468 & $-.772(* *)$ & $.855(* *)$ \\
\hline WIACC range_A & -0.547 & -0.539 & 0.527 & -0.019 & $-.790(* *)$ & -0.503 & 0.539 & $.675\left(^{*}\right)$ & $-.723\left(^{*}\right)$ & -0.535 & $.697(*)$ \\
\hline $\begin{array}{c}\text { WIACC } \\
\text { P90-P10_A } \\
\end{array}$ & $-.634\left(^{*}\right)$ & -0.608 & 0.596 & -0.201 & $-.701\left(^{*}\right)$ & $-.632\left(^{*}\right)$ & 0.608 & $.671(*)$ & $-.762\left(^{*}\right)$ & -0.604 & $.711(*)$ \\
\hline $\begin{array}{l}\text { IACC } \\
\text { A_Global } \\
\end{array}$ & 0,352 & 0,378 & $-0,329$ & $-0,236$ & 0,541 & 0,311 & $-0,378$ & $-0,205$ & 0,046 & 0,355 & $-0,384$ \\
\hline LAeq & -0.474 & -0.442 & 0.491 & -0.370 & 0.024 & -0.515 & 0.442 & 0.456 & -0.146 & -0.468 & 0.382 \\
\hline
\end{tabular}

* Correlación significativa al nivel de 0.05 (bilateral)

** Correlación significativa al nivel de 0.01 (bilateral) 
En referencia al parámetro $\tau_{\text {IACC }}$, se encontró una correlación alta entre el rango de $\tau_{\mathrm{IACC}}$ y los atributos Agradable, Caótico, Calmo, Molesto, Agitado, así como con los componentes principales Pleassantness y Eventfulness, todos estos bajo un nivel de significancia de 0,01. Además, se encontró correlación moderada entre el $\tau_{\text {IACC Range }} \mathrm{y}$ el atributo Uniforme/sin eventos.

La correlación entre $\tau_{\mathrm{IACC} \_ \text {Range }} \mathrm{y}$ la evaluación general del entorno acústico es negativa, lo que implica que cuanto mayor fuera la variación de las fuentes sonoras en el plano horizontal, menor era la evaluación general. Este fenómeno también se repitió en los atributos Agradable, Uniforme/sin eventos, Calmo y el componente Pleassantness. Por otra parte, la correlación entre los atributos

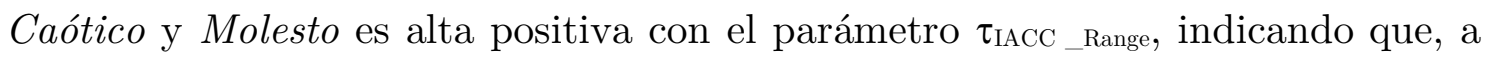
mayores variaciones espaciales de las fuentes sonoras, mayor percepción de caos y molestia. El atributo Agitado y el componente principal Eventfulness presentaron una correlación alta con el parámetro $\tau_{\text {IACC _Range }}$ bajo una significancia de 0.01 , de tal forma que con una probabilidad del $99 \%$ estas variables presentan una alta correlación.

Para las señales con ponderación A se encontró una correlación alta entre el parámetro $\tau_{\text {IACC _Range }}$ y el atributo Agitado y el componente principal Eventfulness (tabla-2-13). Al usar el estadístico descriptivo de diferencias de percentiles 90-10 en el parámetro $\tau_{\text {IACC }}$ se encontraron correlaciones idénticas con los atributos perceptuales mencionados para $\tau_{\text {IACC_range, }}$ es decir, altas correlaciones entre este parámetro y los atributos Agradable, Caótico, Uniforme/sin eventos, Calmo, Molesto, Agitado, los componentes principales Pleassantness y Eventfulness y la evaluación general del entorno acústico, todos a un nivel de 0.01 bilateral.

Al analizar los resultados de correlación entre $\tau_{\text {IACC__ }}$ 90-10_A y los atributos perceptuales (tabla 2-13), fueron encontradas altas correlaciones con los atributos Agradable, Caótico, Calmo, Molesto, Agitado y los componentes Pleassantness y Eventfulness, también a un nivel de significancia de 0.01.

Pasando al parámetro WIACC, usado también para el estudio de la localización espacial en el plano horizontal, se encontró una correlación alta negativa entre los descriptores Rango y diferencia de Percentiles 90-10 con los atributos Uniforme/sin eventos y Monótono. Esto implica nuevamente que, a 
mayores variaciones de las fuentes sonoras en el espacio, los entornos sonoros son percibidos menos monótono y sin eventos. Comportamientos similares fueron encontrados en las señales con ponderación A, donde además de las correlaciones altas negativas entre el WIACC_Range y los atributos Uniforme y Monótono, se encontró también correlación alta positiva con el parámetro Agitado.

No fueron encontradas correlaciones de ningún tipo bajo ningún valor de significancia para correlaciones cruzadas interaurales IACC obtenidas de la IACF largo término.

Respecto al parámetro Leq, los resultados presentan altas correlaciones negativas entre este parámetro y la Evaluación General, los atributos Placentero y Calmo, así como a los componentes principales Pleasantess y Eventfulness. Así mismo se detectaron correlaciones positivas entre el Leq y atributos como Caótico, Desagradable y Agitado, todos con un nivel de significancia de 0.01 (Tabla 2-12). Estos resultados van en la misma línea de trabajos previos donde la molestia se asocia con altos niveles de presión sonora, mientras que lugares con menores niveles de energía son juzgados como más agradables $[6,10]$. No se detectó ningún tipo de correlación bajo ningún nivel de significancia entre el LeqA y los atributos perceptuales (Tabla 2-13).

\subsubsection{Correlación de atributos perceptuales con parámetros temporales derivados de la $A C F$.}

Pasando al estudio de correlación entre parámetros acústicos temporales derivados de la ACF y los atributos perceptuales, las tablas 2-14 y 2-15 presentan los coeficientes de correlación de Spearman tanto para las señales con ponderación $\mathrm{Z}$ como con ponderación A.

Para las señales con ponderación $\mathrm{Z}$ no fueron encontradas correlaciones de ningún tipo bajo ningún valor de significancia entre los parámetros asociados a la ACF y los atributos perceptuales (Tabla 2-14).

Al aplicar el filtro de ponderación A a las señales, fueron encontradas correlaciones moderadas entre el parámetro $\tau_{\mathrm{e} \_ \text {range_A }}$ y los atributos Calmo, la evaluación general y el componente Eventfulness. También se encontró correlación alta entre este parámetro y el atributo Monótono (tabla 2-15). Las correlaciones 
entre el descriptivo $\tau_{\mathrm{e} \_ \text {range_A }}$ y los atributos Calmo, Monótono y la evaluación general son negativas, lo que quiere decir que cuanto mayores son los valores de $\tau_{\mathrm{e} \_ \text {range_A }}$, menores serán los valores medios de los atributos mencionados. La correlación entre el parámetro $\tau_{\mathrm{e} \_ \text {range_A }}$ y el componente Eventfulness es positiva, lo que quiere decir que al incrementar los valores de $\tau_{\mathrm{e} \_ \text {range_A }}$ también lo hace el componente Eventfulness. Para comprender este fenómeno es importante recordar que el parámetro $\tau_{\mathrm{e}}$ se puede interpretar como una especie de reverberación de la señal, representando la auto-semejanza temporal de las señales de audio, por lo que cuanto mayor sea el valor de $\tau_{\mathrm{e}}$, más parecida es la señal respecto a ella misma (más alta la autocorrelación). Por lo anterior se encuentra que cuanto mayor eran las variaciones temporales de las señales, menor era la evaluación general del lugar, así como menos calmo y monótono. Por el contrario, a mayores variaciones temporales producto de grandes rangos de $\tau_{\mathrm{e}}$, más alta era el valor del componente principal Evenfulness (relacionado con lo agitado y lleno de eventos del lugar). Un idéntico comportamiento fue encontrado con el descriptor $\tau_{\mathrm{e} \_ \text {max } \_ \text {A }}$.

Llama la atención el cambio de resultados entre las señales con ponderación Z y las señales con ponderación A para el parámetro $\tau_{e}$. En el primer caso no son detectadas correlaciones entre aspectos objetivos y subjetivos, mientras que en el segundo si se detectan correlaciones altas y moderadas. Este fenómeno se debe al fuerte aporte energético en bajas frecuencias realizado por fuentes vehiculares, los cuales pueden enmascarar la energía aportada por otro tipo de fuentes. Al aplicar la ponderación A a las señales, gran parte de este aporte energético en bajas frecuencias es minimizado, permitiendo apreciar las variaciones temporales en el resto del espectro.

Por último, no fueron encontradas correlaciones de ningún tipo bajo ningún valor de significancia para tiempos efectivos de duración de largo término. 
Segundo Capítulo. Análisis de aspectos espaciales y temporales en la evaluación de paisajes sonoros

Tabla 2-14. Coeficiente de correlación de Spearman entre atributos perceptuales y parámetros temporales con ponderación Z.

\begin{tabular}{|c|c|c|c|c|c|c|c|c|c|c|c|}
\hline & $\begin{array}{c}\text { Evaluación } \\
\text { general }\end{array}$ & Agradable & Caótico & $\begin{array}{l}\text { Excitante/ } \\
\text { Vibrante }\end{array}$ & $\begin{array}{l}\text { Uniforme/ } \\
\text { sin eventos }\end{array}$ & Calmo & Desagradable & Agitado & Monótono & Pleasantness & Evenfulness \\
\hline$\tau_{\mathrm{e} \_ \text {mean }}$ & -0.055 & -0.079 & 0.067 & 0.105 & -0.48 & 0.006 & 0.079 & -0.067 & -0.134 & -0.073 & 0.079 \\
\hline$\tau_{\mathrm{e} \text { median }}$ & 0.073 & 0.055 & -0.079 & 0.216 & -0.334 & 0.115 & -0.055 & -0.213 & -0.055 & 0.067 & -0.055 \\
\hline$\tau_{\mathrm{e} \_\min }$ & 0.14 & 0.103 & -0.127 & 0.309 & -0.353 & 0.224 & -0.103 & -0.195 & -0.292 & 0.116 & -0.055 \\
\hline$\tau_{\mathrm{e} \_\max }$ & -0.091 & -0.127 & 0.164 & -0.049 & -0.462 & -0.03 & 0.127 & -0.012 & -0.043 & -0.146 & 0.139 \\
\hline$\tau_{\mathrm{e} \_\mathrm{p} 10}$ & 0.328 & 0.285 & -0.297 & 0.457 & -0.219 & 0.418 & -0.285 & -0.407 & 0.03 & 0.292 & -0.261 \\
\hline$\tau_{\mathrm{e} \_\mathrm{p} 90}$ & 0.091 & 0.079 & -0.103 & 0.432 & -0.559 & 0.139 & -0.079 & -0.128 & -0.255 & 0.091 & 0.042 \\
\hline$\tau_{\mathrm{e} \_ \text {range }}$ & 0.012 & -0.018 & 0.091 & -0.167 & -0.237 & 0.067 & 0.018 & -0.097 & 0.116 & -0.055 & -0.018 \\
\hline$\tau_{\mathrm{e} \_\mathrm{p} 90 \_\mathrm{p} 10}$ & 0.109 & 0.127 & -0.188 & 0.253 & -0.334 & 0.067 & -0.127 & -0.122 & -0.207 & 0.158 & 0.006 \\
\hline$\tau_{\mathrm{e} \_ \text {global }}$ & 0.201 & 0.152 & -0.164 & 0.315 & -0.31 & 0.285 & -0.152 & -0.316 & -0.085 & 0.158 & -0.127 \\
\hline
\end{tabular}

* Correlación significativa al nivel de 0.05 (bilateral)

** Correlación significativa al nivel de 0.01 (bilateral) 
Segundo Capítulo. Análisis de aspectos espaciales y temporales en la evaluación de paisajes sonoros

Tabla 2-15. Coeficiente de correlación de Spearman entre atributos perceptuales y parámetros temporales con ponderación A.

\begin{tabular}{|c|c|c|c|c|c|c|c|c|c|c|c|}
\hline & $\begin{array}{c}\text { Evaluación } \\
\text { general }\end{array}$ & Agradable & Caótico & $\begin{array}{l}\text { Excitante/ } \\
\text { Vibrante }\end{array}$ & $\begin{array}{l}\text { Uniforme/ } \\
\text { sin eventos }\end{array}$ & Calmo & Desagradable & Agitado & Monótono & Pleasantness & Evenfulness \\
\hline$\tau_{\mathrm{e} \_ \text {meanA }}$ & -0.409 & -0.395 & 0.407 & -0.186 & -0.415 & -0.395 & 0.395 & 0.317 & -0.591 & -0.402 & 0.401 \\
\hline$\tau_{\mathrm{e} \_ \text {medianA }}$ & -0.249 & -0.224 & 0.248 & -0.445 & -0.134 & -0.285 & 0.224 & 0.231 & -0.486 & -0.237 & 0.236 \\
\hline$\tau_{\mathrm{e} \_\min A}$ & 0.03 & 0.043 & -0.024 & -0.44 & 0.284 & -0.03 & -0.043 & -0.034 & 0.195 & 0.034 & -0.079 \\
\hline$\tau_{\mathrm{e} \_\max A}$ & $-.638\left(^{*}\right)$ & -0.612 & 0.624 & -0.228 & -0.559 & $-.648\left(^{*}\right)$ & 0.612 & 0.614 & $-.711(*)$ & -0.62 & $.685\left(^{*}\right)$ \\
\hline$\tau_{\mathrm{e} \_\mathrm{p} 10 \mathrm{~A}}$ & 0 & 0.012 & 0.012 & -0.245 & 0.159 & -0.024 & -0.012 & 0.043 & -0.159 & 0 & 0 \\
\hline$\tau_{\mathrm{e} \_\mathrm{p} 90 \mathrm{~A}}$ & -0.353 & -0.333 & 0.345 & -0.235 & -0.316 & -0.333 & 0.333 & 0.407 & -0.492 & -0.34 & 0.358 \\
\hline$\tau_{\mathrm{e} \_ \text {rangeA }}$ & $-.638\left(^{*}\right)$ & -0.612 & 0.624 & -0.228 & -0.559 & $-.648\left(^{*}\right)$ & 0.612 & 0.614 & $-.711(*)$ & -0.62 & $.685(*)$ \\
\hline $\begin{array}{c}\tau_{\mathrm{e}} \\
\_\mathrm{P} 90 \_\mathrm{P} 10 \mathrm{~A}\end{array}$ & -0.304 & -0.273 & 0.236 & -0.031 & -0.359 & -0.297 & 0.273 & 0.389 & -0.584 & -0.255 & 0.333 \\
\hline
\end{tabular}

* Correlación significativa al nivel de 0.05 (bilateral)

** Correlación significativa al nivel de 0.01 (bilateral) 


\subsection{Discusión y conclusiones.}

El estudio y evaluación de paisajes sonoros urbanos es un proceso multidisciplinar que requiere de aspectos objetivos, subjetivos y descriptivos con el fin de alcanzar una visión holística de los lugares en estudio. En este capítulo fue usada una combinación de herramientas descriptivas, parámetros objetivos y atributos perceptuales para analizar diversos paisajes sonoros, con especial énfasis en la influencia de aspectos temporales y espaciales en las respuestas de los oyentes.

Los resultados de esta investigación indican que aspectos relacionados con las variaciones de las fuentes sonoras en el plano horizontal pueden impactar en las respuestas de los oyentes. Más específicamente el parámetro $\tau_{\mathrm{IACC}}$ (y en menor medida $\mathrm{W}_{\mathrm{IACC}}$ ), obtenido de la IACF móvil permite apreciar variaciones espaciales de las fuentes sonoras en el plano horizontal y su impacto en las respuestas de los oyentes. Para este fin es clave encontrar y escoger descriptores estadísticos que permitan apreciar estas variaciones. Es por esto que descriptores estadísticos como Rango o diferencias de percentiles 90-10 son indicadores recomendados en el estudio espacial de paisajes sonoros.

Así mismo, a partir de las pruebas de correlación de Spearman, los resultados encontrados sugieren que la mayoría de los atributos perceptuales que contempla el modelo de Axelsson, sus componentes principales (Eventfulness y Pleasantness) e incluso la impresión general del entorno acústico tienen alcances espaciales poco explorados. El parámetro $\tau_{\text {IACC }}$ presentó en alguno de sus descriptivos estadísticos altos grados de correlación con distintos aspectos subjetivos, encontrándose mayor influencia de las variaciones espaciales en atributos como Agitado, Calmo y Molesto, esto sin importar si la señal tenía ponderación A o Z . Considerando que $\tau_{\mathrm{IACC}}$ está relacionado con la ubicación de la fuente sonora en el plano horizontal, los resultados sugieren que descriptores estadísticos como la diferencia de percentil 90-10 o el rango pueden ser útiles en el análisis de respuestas relacionadas con el grupo de atributos de ActividadDiversidad presentados en la tabla 1-1 del primer capítulo. Un comportamiento similar, aunque en un menor número de atributos perceptuales fue encontrado en el parámetro WIACC. En consecuencia, los resultados obtenidos para los 10 lugares en estudio revelan una relación entre la variación de las fuentes de sonido en el 
plano horizontal y los dos grupos de atributos perceptivos de los paisajes sonoros (Placer/Confort y Actividad/Diversidad). Lo anterior demuestra que la agitación, la variabilidad, el caos o la uniformidad de los entornos acústicos pueden ser percibidos no solo a partir de variaciones energéticas, sino también de variaciones de la ubicación espacial en el plano horizontal de las fuentes sonoras.

Aunque algunos estudios han empleado parámetros derivados de la IACF para describir las fuentes sonoras y entornos acústicos [39-41], y otros han analizado la relación entre la molestia y las fluctuaciones espaciales de las fuentes de sonido $[42,43]$, esta investigación ofrece información detallada sobre el estudio de parámetros espaciales y la evaluación subjetiva de paisajes sonoros. Para los casos de estudio presentados en este trabajo, paisajes sonoros calificados como más agradables presentaron correlaciones negativas con los parámetros $\tau_{\text {IACC_Range }}$ y $\tau_{\text {IACC } \_90-10}$. Esto quiere decir que, como se mencionó en la sección 2.3.3, al presentar mayores rangos y diferencias percentiles en la ubicación horizontal de las fuentes sonoras lo lugares eran considerados menos agradables. Este resultado está en la misma línea que los trabajos desarrollados por Sato et. al, quienes encontraron que mayores variaciones de $\tau_{\text {IACC }}$ en señales sonoras eran consideradas eran más molestos [42]. Los resultados del trabajo de Sato et. al. también incluyen que fuertes variaciones de IACC eran consideradas molestas, aunque en los resultados obtenidos en esta tesis, dicha correlación no fue encontrada.

En lo que respecta a los parámetros asociados a la $\mathrm{ACF}$, aunque los resultados obtenidos no fueron tan contundentes como con los de la IACF, también fueron encontradas correlaciones entre aspectos objetivos y subjetivos. El rango de $\tau_{\text {e }}$ presentó una alta correlación negativa con el parámetro Monótono, así como correlaciones positivas moderadas con los atributos Agitado y el componente principal Eventfulness, todos asociados al grupo de atributos Actividad- Diversidad de la tabla 1-1. Esto quiere decir que la percepción de atributos que se relacionan con la variabilidad de las señales sonoras puede ser descrita con parámetros acústicos especializados en el estudio de la autosemejanza de señales.

Comparando los resultados obtenidos en esta tesis para el parámetro $\tau_{\mathrm{e}}$ con los presentados por Fujii et. al, donde se aplicaron parámetros relacionados con la $\mathrm{ACF}$ en el estudio de molestia tráfico rodado encontrando correlación positiva 
entre la variación $\tau_{\mathrm{e}}$ y la molestia generada [44], en esta tesis no fue encontrada ninguna correlación bajo ningún nivel de significancia.

Es interesante hacer un análisis específico de lugares con sonidos fundamentales de fuentes de agua (casos de BRFA y BOFAET) y su comportamiento de $\tau_{\mathrm{e}}$. Los resultados de la evaluación subjetiva para estos dos lugares en el atributo Uniforme/sin eventos fueron los más altos de los diez lugares en estudio, aunque los valores de $\tau_{\mathrm{e}}$ en las medidas de tendencia central (medias y medianas) eran los más bajos. Esto se debe a que si bien las características aleatorias de las fuentes de agua (similares a un ruido rosa) generan bajos valores de $\tau_{\mathrm{e}}$ (la señal no se parece a ella misma respecto al tiempo debido a su constante renovación), aunque la percepción que se tiene es de un lugar sin eventos. En este sentido se considera que las fuentes de agua presentan son un claro ejemplo de que la constante renovación de las señales acústicas no necesariamente impacta en la percepción de uniformidad de entornos acústicos.

Los resultados obtenidos presentan una mayor potencia en los parámetros acústicos binaurales que en los temporales. Variaciones de la ubicación en el plano horizontal de las fuentes sonoras son asociadas con un gran número de atributos perceptuales que indican movimiento de las fuentes sonoras. Si bien fueron encontradas correlaciones entre parámetros acústicos temporales y atributos perceptuales, el número de casos que presentaron correlación fue mucho menor.

No se encontraron resultados que sugieran que el uso de las ACF e IACF de largo término pueden ser útiles, bien sea en la descripción de entornos acústicos o en el análisis de aspectos subjetivos. Este resultado era esperada dado que en un paisaje sonoro urbano existirán continuas variaciones de las fuentes sonoras en su comportamiento espacial respecto al tiempo, las cuales no son detectadas por las funciones de largo término. Es por esto que, para el estudio de entornos acústicos, se recomienda el uso de las ACF e IACF en sus versiones móviles.

En relación al parámetro Leq, se observaron correlaciones negativas entre dicho parámetro y la Evaluación general y los atributos Agradable y Calmo, lo cual es consistente con la correlación positiva identificada entre este descriptor acústico y los atributos Desagradable y Agitado. Los resultados de esta investigación concuerdan con los hallazgos de trabajos previos, que han demostrado que los parámetros asociados con los niveles de energía influyen en el 
grado de confort y/o molestia [3,6,10,28,30]. Sin embargo, para atributos como Monótono o Sin eventos, no se encontraron correlaciones en ningún nivel de significancia. Este resultado puede explicarse por el hecho de que el parámetro Leq representa un promedio energético temporal, lo que significa que no es sensible a los atributos que representan las variaciones de la fuente de sonido.

Asimismo, es útil comparar los resultados de las señales con ponderación A para los parámetros energéticos y binaurales. No se encontró correlación entre LAeq y los atributos perceptuales, mientras que, para varios parámetros binaurales, se observaron diferentes tipos de correlación (Tablas 2-13). Estos resultados pueden deberse al hecho de que los 10 paisajes sonoros estudiados se encuentran en un entorno urbano, donde la contribución energética a bajas frecuencias de las fuentes de tráfico vehicular es importante. Por lo tanto, estos resultados permiten apreciar la sensibilidad del parámetro de energía en relación a las bajas frecuencias.

Cuando se discuten estos resultados, es clave recordar que están limitados a una muestra de 10 paisajes sonoros urbanos (comunes). Algunos de los casos específicos estudiados incluyeron parques y plazas con fuentes de agua. Tales lugares desempeñan un papel importante en las ciudades, ya que son áreas de tranquilidad que ofrecen a los usuarios espacios de descanso y relajación. Los resultados de las evaluaciones subjetivas para estos casos muestran que estos sitios se encuentran en el cuadrante inferior derecho del modelo de Axelsson (lugares agradables con pocos eventos), lo que concuerda con los hallazgos de investigaciones anteriores [6]. Además, esta investigación evidenció que los lugares con sonidos naturales presentaron menos diversidad de fuentes de sonido y poca variación espacial en el plano horizontal. Toda esta información permite conectar la evaluación subjetiva, los resultados objetivos y los aspectos descriptivos.

A la luz de estos hallazgos, se puede concluir que la descripción y el análisis de los entornos acústicos deben considerar aspectos energéticos y descriptivos, así como la variabilidad temporal y espacial de las fuentes de sonido en el plano horizontal. Este análisis, junto con los aspectos perceptivos, permitirá un enfoque analítico amplio de los paisajes sonoros urbanos. 


\section{Referencias Segundo Capítulo.}

[1] Genuit K., A. Fiebig. Psychoacoustics and its benefit for the soundscape approach. Acta Acustica United with Acustica 2006;92:952-8.

[2] Hall D.A., A. Irwin, M. Edmondson-Jones, S. Phillips, J.E.W. Poxon. An exploratory evaluation of perceptual, psychoacoustic and acoustical properties of urban soundscapes. Applied Acoustics 2013;74:248-54. doi:10.1016/j.apacoust.2011.03.006.

[3] Maristany A., M. Recuero López, C. Asencio Rivera. Soundscape quality analysis by fuzzy logic: A field study in Cordoba, Argentina. Applied Acoustics 2016;111:106-15. doi:10.1016/j.apacoust.2016.04.013.

[4] Szeremeta B., P.H.T. Zannin. Analysis and evaluation of soundscapes in public parks through interviews and measurement of noise. Science of the Total Environment 2009;407:6143-9. doi:10.1016/j.scitotenv.2009.08.039.

[5] UNE-ISO. UNE-ISO 1996-1 Descripción, medición y evaluación del ruido ambiental. Parte 1: Magnitudes básicas y métodos de evaluación. 2005.

[6] Axelsson Ö., M.E. Nilsson, B. Berglund. A principal components model of soundscape perception. The Journal of the Acoustical Society of America 2010;128:2836-46. doi:10.1121/1.3493436.

[7] Cain R., P. Jennings, J. Poxon. The development and application of the emotional dimensions of a soundscape. Applied Acoustics 2013;74:232-9. doi:10.1016/j.apacoust.2011.11.006.

[8] Guillén J., I. López. Importance of personal, attitudinal and contextual variables in the assessment of pleasantness of the urban sound environment. 19th Int. Congr. Acoust. ICA, Madrid: 2007, p. 1-6.

[9] Kawai K., T. Kojima, K. Hirate, M. Yasuoka. Personal evaluation structure of environmental sounds: Experiments of subjective evaluation using subjects' own terms. Journal of Sound and Vibration 2004;277:523-33. doi:10.1016/j.jsv.2004.03.013.

[10] Jeon J.Y., P.J. Lee, J. You, J. Kang. Perceptual assessment of quality of urban soundscapes with combined noise sources and water sounds. The Journal of the Acoustical Society of America 2010;127:1357-66. doi:10.1121/1.3298437.

[11] Jeon J.Y., P.J. Lee, J.Y. Hong, D. Cabrera. Non-auditory factors affecting urban soundscape evaluation. The Journal of the Acoustical Society of America 2011;130:3761-70. doi:10.1121/1.3652902.

[12] Ando Y., P. Cariani. Auditory and visual sensations. London: Springer; 2009. doi:10.1007/b13253.

[13] Kaieda S., K. Kawai, T. Yano, Y. Ando. A study on measures of timbre of 
electric guitar sounds in terms of power spectrum and auto correlation function. Journal of Temporal Design 2009;9:43-6.

[14] Bidondo A. AdAA 2009 Re-descubriendo la ventana de integración temporal del sistema auditivo : consecuencias en la ciencia Acústica 2009:118.

[15] Ando Y. Architectural Acoustics: Blending Sound Sources, Sound Fields, and Listeners. vol. 104. First. Springer; 1998. doi:10.1159/000267225.

[16] Ando Y. Opera House Acoustics Based on Subjective Preference Theory. Kobe-Japan: Springer Japan; 2015. doi:10.1007/978-4-431-55423-3.

[17] Damaske P., Y. Ando. Interaural Crosscorrelation for Multichannel Loudspeaker Reproduction. Acustica: Spatial Sound Techniques: Part 2 1972;27:232-8.

[18] Axelsson Ö. Introducing soundscape. AESOP 26th Annual Congress 2012.

[19] Axelsson Ö. How to measure soundscape quality. Proceeding Euronoise 2015, Maastrícht: EAA-NAG-ABAV; 2015, p. 1477-81.

[20] Axelsson Ö. Aesthetic Appreciation Explicated. 2011.

[21] Axelsson Ö., M.E. Nilsson, B. Berglund. The Swedish soundscape-quality protocol. Journal of Acoustical Society of America 2012;131:3476. doi:http://dx.doi.org/10.1121/1.4709112.

[22] Schafer R.M. The Tuning of the World. Random House; 1977. doi: $10.2307 / 3345272$.

[23] Brown A.L., T. Gjestlan, D. Dubois. Acoustic Environments and soundscapes. In: Kang J, Schulte-Fortkamp B, editors. Soundscape Built Environ., 2016, p. 1-16.

[24] Hong J.Y., J.Y. Jeon. Designing sound and visual components for enhancement of urban soundscapes. The Journal of the Acoustical Society of America 2013;134:2026-36. doi:10.1121/1.4817924.

[25] Viollon S., C. Lavandier, C. Drake. Influence of visual setting on sound ratings in an urban environment. Applied Acoustics 2002;63:493-511. doi:10.1016/S0003-682X(01)00053-6.

[26] Hume K., M. Ahtamad. Physiological responses to and subjective estimates of soundscape elements. Applied Acoustics 2013;74:275-81. doi:10.1016/j.apacoust.2011.10.009.

[27] Pheasant R., K. Horoshenkov, G. Watts, B. Barrett. The acoustic and visual factors influencing the construction of tranquil space in urban and rural environments tranquil spaces-quiet places? The Journal of the Acoustical Society of America 2008;123:1446-57. doi:10.1121/1.2831735.

[28] Rey Gozalo G., J. Trujillo Carmona, J.M. Barrigón Morillas, R. VílchezGómez, V. Gómez Escobar. Relationship between objective acoustic indices 
and subjective assessments for the quality of soundscapes. Applied Acoustics 2015;97:1-10. doi:10.1016/j.apacoust.2015.03.020.

[29] Marry S., J. Defrance. Analysis of the perception and representation of sonic public spaces through on site survey, acoustic indicators and in-depth interviews. Applied Acoustics 2013;74:282-92. doi:10.1016/j.apacoust.2012.01.005.

[30] Ricciardi P., P. Delaitre, C. Lavandier, F. Torchia, P. Aumond. Sound quality indicators for urban places in Paris cross-validated by Milan data. The Journal of the Acoustical Society of America 2015;138:2337-48. doi:10.1121/1.4929747.

[31] Sato S., Y. Ando. Apparent Source Width ( ASW ) of Complex Noises in Relation to the Interaural Cross-correlation Function. Journal of Temporal Design in Architecture and the Environment 2002;2:29-32.

[32] Sato S.I., S. Wu. Comparison of different calculation methods of effective

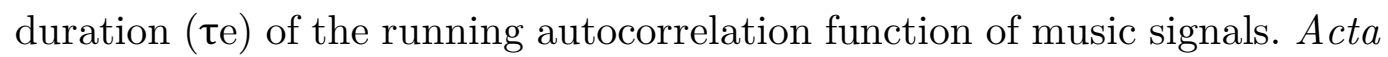
Acustica United with Acustica 2011;97:432-40. doi:10.3813/AAA.918424.

[33] Hermida L., I. Pavón. Spatial aspects in urban soundscapes: Binaural parameters application in the study of soundscapes from Bogotá-Colombia and Brasília-Brazil. Applied Acoustics 2019;145:420-30. doi:10.1016/j.apacoust.2018.10.011.

[34] Otto N., S. Amman, C. Eaton, S. Lake. Guidelines for Jury Evaluations of Automotive Sounds. Sound and Vibration 2001;April:1-14. doi:1999-011822.

[35] Otto N.C. Listening test methods for automotive sound quality. Audio Eng. Soc., New York: Audio engineering Society; 1997.

[36] Otto N., S. Amman, C. Eaton, S. Lake. Guidelines for Jury Evaluations of Automotive Sounds. vol. April. 1999. doi:1999-01-1822.

[37] Lyon R.H. Product sound quality: From perception to design. Sound and Vibration 2003;108:2471. doi:10.1121/1.4743110.

[38] ANSI/ASA. ANSI/ASA S12.2-2008. Criteria for Evaluating Room Noise 2008.

[39] Soeta Y., R. Shimokura. Survey of interior noise characteristics in various types of trains. Applied Acoustics 2013;74:1160-6. doi:10.1016/j.apacoust.2013.04.002.

[40] Jeon J.Y., P.J. Lee, J.H. Kim, S.Y. Yoo. Subjective evaluation of heavyweight floor impact sounds in relation to spatial characteristics. The Journal of the Acoustical Society of America 2009;125:2987. doi:10.1121/1.3081390.

[41] Kitamura T., R. Shimokura, S. Sato, Y. Ando. Measurement of temporal 
and spatial factors of a flushing toilet noise in a downstairs bedroom. Journal of Temporal Design in Architecture and the Environment $2002 ; 2: 13-9$.

[42] Sato S.I., T. Kitamura, Y. Ando. Annoyance of noise stimuli in relation to the spatial factors extracted from the interaural cross-correlation function. Journal of Sound and Vibration 2004;277:511-21. doi:10.1016/j.jsv.2004.03.012.

[43] Soeta Y., Y. Ando. Neurally Based Measurement and Evaluation of Environmental Noise. Tokio: Springer; 2016.

[44] Fujii K., J. Atagi, Y. Ando. Temporal and Spatial Factors of Traffic Noise and Its Annoyance. Journal of Temporal Design in Architecture and the Environment 2002;2:33-41. 


\section{CAPÍTULO 3.}

\section{PAISAJES SONOROS: RESPUESTAS Y SALIDAS Y SU RELACIÓN CON EL CONTEXTO.}




\section{PAISAJES SONOROS: RESPUESTAS Y SALIDAS Y SU RELACIÓN CON EL CONTEXTO.}

Como se ha mencionado a lo largo de todo este documento, el estudio y evaluación de paisajes sonoros requiere de un enfoque interdisciplinar, donde además de abordar aspectos de caracterización de los entornos acústicos, también debe realizarse un análisis de tipo subjetivo [1-3]. En este sentido, la norma ISO 12913-1 especifica que el paisaje sonoro solo existe a través de la percepción humana de los entornos sonoros [4], por lo que los trabajos desarrollados en este campo deben incluir elementos objetivos, subjetivos y contextuales. Bajo este enfoque, esta tesis doctoral se ha desarrollado considerando estos tres elementos, por lo que la primera parte de esta tesis se enfocó en el estudio de entornos acústicos a partir de aspectos temporales y espaciales, mientras que esta sección trata aspectos perceptuales y contextuales de los paisajes sonoros.

La percepción está mediada por aspectos socioculturales y psicológicos, los cuales a su vez se ven condicionados por el contexto [5,6]. En el marco conceptual propuesto por la norma ISO 12913-1 el contexto influencia prácticamente todos los componentes del marco conceptual de paisajes sonoros (Figura 1-4), condicionando de esta forma las respuestas y salidas de las personas, aunque como lo remarca Brown, la especificación de los aspectos que deben considerados en el análisis contextual sigue siendo difuso [7]. Si bien la norma ISO 12913-2 presenta un avance en cuanto a toma y presentación de datos (principalmente objetivos, descriptivos y de respuestas a corto plazo) [8], el estudio de la influencia de aspectos contextuales aun requiere de esfuerzos para determinar su impacto en respuestas y salidas de los oyentes. Considerando las respuestas y salidas de los oyentes se relacionan con las experiencias y usos de las personas en los espacios urbanos, puede decirse que el diseño, la planeación y la gestión de paisajes sonoros no solo comprometen aspectos puramente ingenieriles, sino que también precisan de enfoques que consideren la percepción ecológica, el enfoque de experiencia ambiental y finalmente de elementos de la comunicación. 
De esta forma, este capítulo del documento está encaminada al estudio de la relación entre contexto y el paisaje sonoro, enfocándose principalmente en el análisis de la influencia de la interacción lugar-persona en la evaluación de paisajes sonoros. En este capítulo se busca dar respuestas a preguntas como:

- ¿Existen diferencias estadísticamente significativas en las respuestas de los oyentes dependiendo del tipo de metodología usada en la evaluación (in situ vs. laboratorio)? ¿Qué aspectos específicos de las respuestas afecta?

- ¿Existen diferencias estadísticamente significativas en las respuestas y salidas en grupos de oyentes con conocimiento y experiencias previas del entorno evaluado? ¿Varia el significado del lugar según el contexto?

- ¿Cual es la influencia de aspectos contextuales y semióticos en procesos de diseño, planeación y gestión de entornos acústicos urbanos?

Para ello fueron realizados dos estudios, el primero relacionado con la comparación entre metodologías de evaluación de paisajes sonoros (in situ vs. laboratorio) y el segundo relacionado con la evaluación en laboratorio de paisajes sonoros teniendo en cuenta diferentes experiencias previas de los oyentes a partir del conocimiento contextual de los lugares en estudio (diferentes interacciones Lugar-Persona).

Este capítulo comienza con una introducción teórica, donde se tratan aspectos perceptuales, elementos de la experiencia ambiental aplicado a paisajes sonoros, así como también aspectos generales de calidad sonora y de semiótica bajo el enfoque de los paisajes sonoros. Así mismo, se realiza una descripción metodológica detallada, se presentan los resultados obtenidos y se abre una discusión que permite llegar a las conclusiones específicas de este capítulo. 


\subsection{Aproximación teórica: percepción ecológica, experiencia ambiental, calidad sonora y comunicación y semiótica en el estudio de paisajes sonoros urbanos.}

\subsubsection{Psicología ecológica y enfoque tradicional de percepción.}

Para poder comprender el proceso de percepción de entornos acústicos, se considera necesario realizar una primera inmersión en teorías básicas perceptivas. En esta sección se presenta la teoría de percepción ecológica, la cual fue propuesta inicialmente para describir percepciones visuales, pero que también aplicada en teorías de diseño. Además, se presenta la teoría tradicional de percepción, la cual se ve influenciada por experiencias previas y el contexto.

Según James Gibson, el hombre se relaciona y percibe su ambiente a partir de los diferentes estímulos que encuentra. El ambiente es el entorno de los animales y en él se pueden encontrar, además de otros animales, plantas y elementos no vivos [9]. En el ambiente, todos esos elementos interactúan, lo que quiere decir que todos los "animales", en menor o mayor medida, son perceptores y percibidos. De esta forma existe un continuum entre el animal y el ambiente, condicionando al entorno como un prerrequisito para la vida animal, por lo que es importante describir y estudiar el ambiente para comprender la percepción del entorno.

Una de las formas de estudiar el ambiente ha sido a partir de modelos físicos, buscando la caracterización del entorno por medio de la delimitación de propiedades y características físicas [10]. Sin embargo, la pregunta hecha a partir del enfoque ecológico es: ¿que es lo que el hombre percibe de su ambiente? El enfoque ecológico busca describir el hábitat de los animales, pues es en él que tanto hombres como animales ven, huelen, tocan y escuchan. Este enfoque se fundamenta en la hipótesis de que el animal no percibe en unidades físicas sino en unidades de ambiente. Esto quiere decir que, bajo este enfoque, la percepción se realiza a partir de un contacto directo entre hombre-ambiente.

Por otra parte, el ambiente puede ser descrito en términos de medio, sustancia y superficie. El medio tiene información que permite al animal moverse, sus características ofrecen variaciones lumínicas, vibraciones mecánicas y la difusión de olores [9]. Toda esta información varía siempre que el animal se mueve, por lo que cada punto del medio es considerado único. Toda esta oferta natural 
que tiene el medio es denominada "Affordance" (lo que el medio ofrece), y según Gibson, es invariante y ha acompañado la evolución animal desde siempre $[11,12]$.

El segundo elemento propuesto en la teoría ecológica para describir el ambiente es la sustancia. La materia en estado sólido o semisólido es substancial, mientras que la materia en estado gaseoso es insubstancial [9]. Es a partir de las sustancias que el animal puede tomar la decisión de moverse, comer o desarrollar objetos que le permitan sobrevivir, por lo cual las sustancias tienen una fuerte influencia en los comportamientos de los animales. Por último, las superficies separan el medio de las sustancias.

De esta forma, según Gibson, el ambiente se encuentra continuamente evaluado por el hombre y esta información le permite tomar decisiones. En otras palabras, es a través del sistema perceptivo que el hombre puede percibir el affordance del medio, moverse y actuar según esta información.

A partir de estas premisas tomadas desde el enfoque ecológico y aplicándolas a los entornos sonoros urbanos, puede verse que existen una gran cercanía entre la percepción ecológica y los paisajes sonoros, pues en los dos conceptos se requiere de un acercamiento directo entre el ser humano y el entorno, enfocándose en la forma en que el ser humano lo percibe. Así mismo, pasando al objeto de estudio de este capítulo, puede formularse la pregunta ¿cómo influye el affordance sonoro de los entornos urbanos en las respuestas y salidas de los oyentes?

Aunque el enfoque ecológico de Gibson ha tenido amplia aceptación, principalmente por la importancia que se le da a la relación directa entre hombreambiente, su propuesta también ha generado gran controversia. En la teoría de percepción ecológica se afirma que los estímulos encontrados en el medio son invariantes, por lo que toda la información necesaria para construir la percepción se encuentra directamente en el estímulo, negando de esta forma la importancia de la experiencia previa del perceptor [13]. Esta postura va en contra del modelo convencional de percepción presentado en la figura 3-1, donde el estímulo llega el órgano sensorial y se convierte en señal nerviosa, la cual es llevada al cerebro y a partir de la experiencia previa en la memoria, el cerebro interpreta, organiza e integra las sensaciones entrantes en percepciones, formulando una respuesta a partir de las percepciones del sujeto $[14,15]$. Bajo este enfoque tradicional de 
percepción, puede decirse que este término se refiere al proceso en que las personas traducen impresiones sensoriales en una visión coherente y definida del mundo que lo rodea [5].

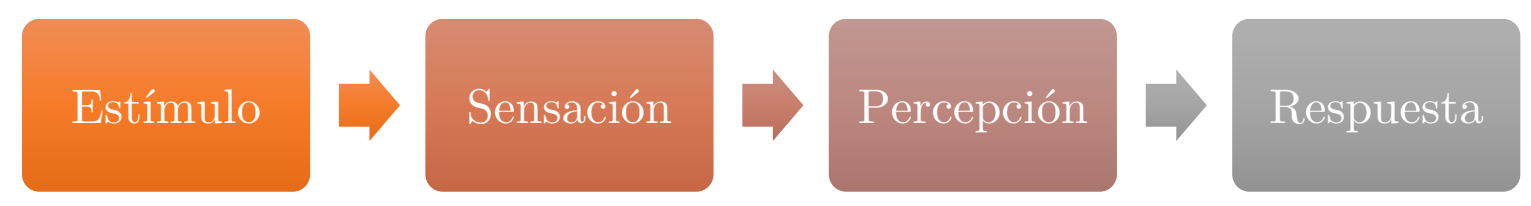

Figura 3-1. Modelo tradicional de percepción (Schiffman, 2001)

Si bien no es objeto de esta tesis debatir acerca de los modelos de percepción humana, se considera importante citar tanto el enfoque de Gibson (debido a la potencia del término affordance y a la necesidad de fortalecer la relación entre hombre-ambiente) como el modelo tradicional de percepción (donde la experiencia previa es importante para la creación de respuestas) a fin de complementar el proceso de percepción de entornos acústicos.

Profundizando en la definición de paisaje sonoro establecida por la norma ISO 12913-1, donde se dice que paisaje sonoro es la percepción, experimentación y/o entendimiento de los entornos acústicos, puede considerarse que el paisaje sonoro ofrece una visión del mundo sonoro que rodea al oyente.

Por otra parte, teniendo en cuenta la distinción marcada en la norma ISO 12913-1 acerca de respuestas y salidas de los oyentes, es necesario fortalecer la diferencia entre estos dos términos, por lo que es importante realizar un abordaje al concepto de experiencia ambiental.

3.1.2 Modelo de experiencia ambiental aplicado a los paisajes sonoros.

\subsubsection{Modelo general de experiencia ambiental.}

Según Herranz-Pascual, la experiencia ambiental se conforma a partir de la relación entre el hombre y el ambiente, donde una gran cantidad de aspectos fisiológicos y psicológicos son accionados, permitiendo recopilar y obtener información del ambiente. Las sensaciones son recibidas e integradas en contenidos y unidades de significado con las que se reconoce, explora y/o se compara el medio ambiente. A partir de esto se experimentan sensaciones o emociones y en consecuencia se actúa de una forma determinada [16]. 
En el modelo de experiencia ambiental los procesos no están fragmentados, por el contrario, todo está totalmente interrelacionado. Dicha interrelación incluye aspectos cognitivos (pensamientos), afectivos (emociones), interpretativos (significados) y evaluativos (actitudes). Por lo anterior pueden ser descritas la percepción ambiental, la cognición ambiental, el significado ambiental, la actitud ambiental y la valoración ambiental como se muestra en la Figura 3-2.

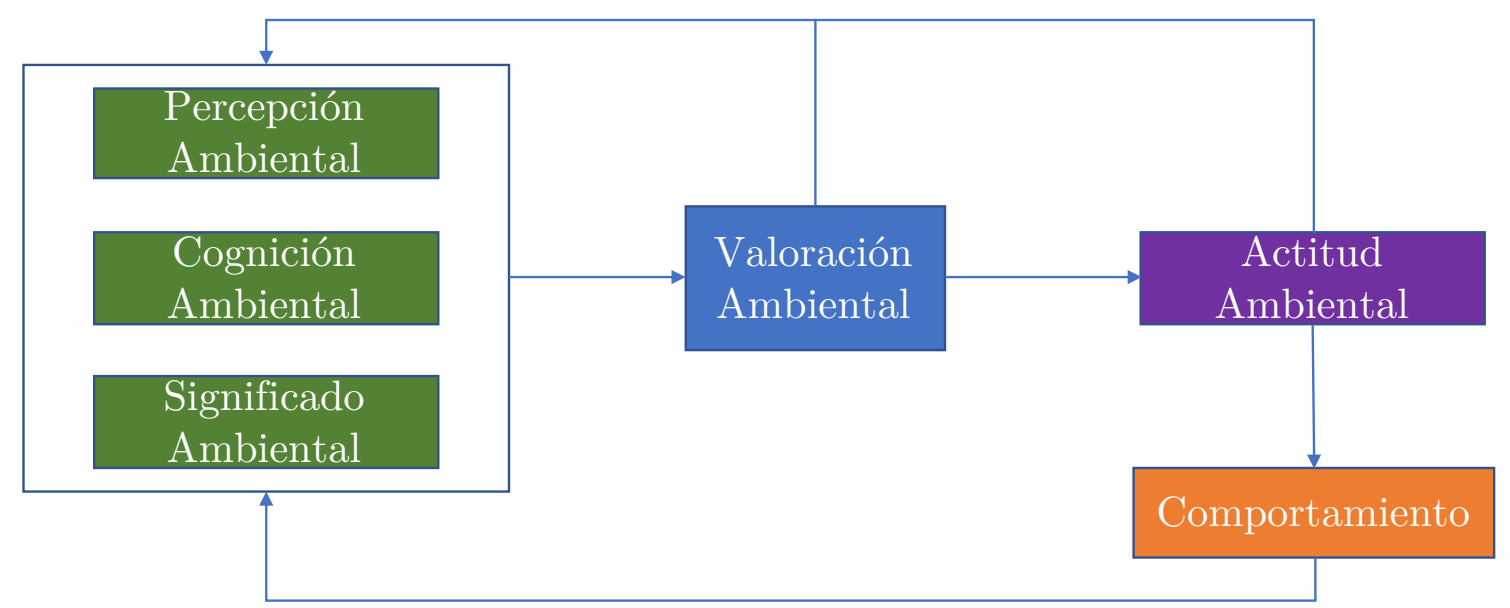

Figura 3-2. Proceso psicológico de experiencia ambiental, (Pol y Valera [17]).

La percepción ambiental se refiere al proceso en que la información sensorial es interpretada y organizada en unidades de significado para configurar una imagen coherente del entorno o de parte de él. La cognición ambiental representa procesos más altos de selección, estructuración, memorización o recuperación de la información percibida en relación con el entorno. El significado ambiental se refiere a aspectos de tipo emocional, afectivo y simbólico de la experiencia ambiental, por lo que en este campo se tratan elementos como experiencias afectivas, apego e identidad con el lugar, etc. La actitud ambiental está relacionada con las actitudes favorables o desfavorables para el medio ambiente en general o hacia una característica específica ${ }^{1}$ [18]. La valoración ambiental es la combinación de evaluaciones referidas a la mezcla de juicios de un grupo de personas que da lugar a opiniones ampliamente compartidas sobre un entorno o lugar específico (evaluación ambiental) o a evaluaciones ambientales más específicas (evaluaciones ambientales) [16,19].

\footnotetext{
${ }^{1}$ La actitud, desde el enfoque de la psicología ambiental, se refiere a la evaluación que las personas hacen sobre otras personas, ideas o cosas que técnicamente reciben la denominación de objetos de actitud. Esto quiere decir que las actitudes se refieren al grado "positivo o negativo con que las personas tienden a juzgar cualquier aspecto de la realidad" [18].
} 
Teniendo en cuenta el modelo psicológico de experiencia ambiental, puede apreciarse la diferencia entre actitudes y comportamientos. Mientras que las actitudes se refieren al grado en que las personas tienden a juzgar aspectos de la realidad, el comportamiento (también llamado conducta) es considerado el conjunto de respuestas o decisiones producto de la relación entre el hombre y el ambiente [20]. Bajo estos conceptos, podrían establecerse relaciones entre actitudes y comportamientos con respuestas y salidas del modelo de paisajes sonoros de la norma ISO 12913-1. Las respuestas pueden relacionarse con actitudes (respuestas a corto plazo) mientras que las salidas pueden relacionarse con comportamientos (consecuencias a largo plazo).

A partir del proceso psicológico de experiencia ambiental de Pol y Valera, Herranz-Pascual et al. proponen el modelo general de experiencias ambientales, presentado en la figura 3-3.

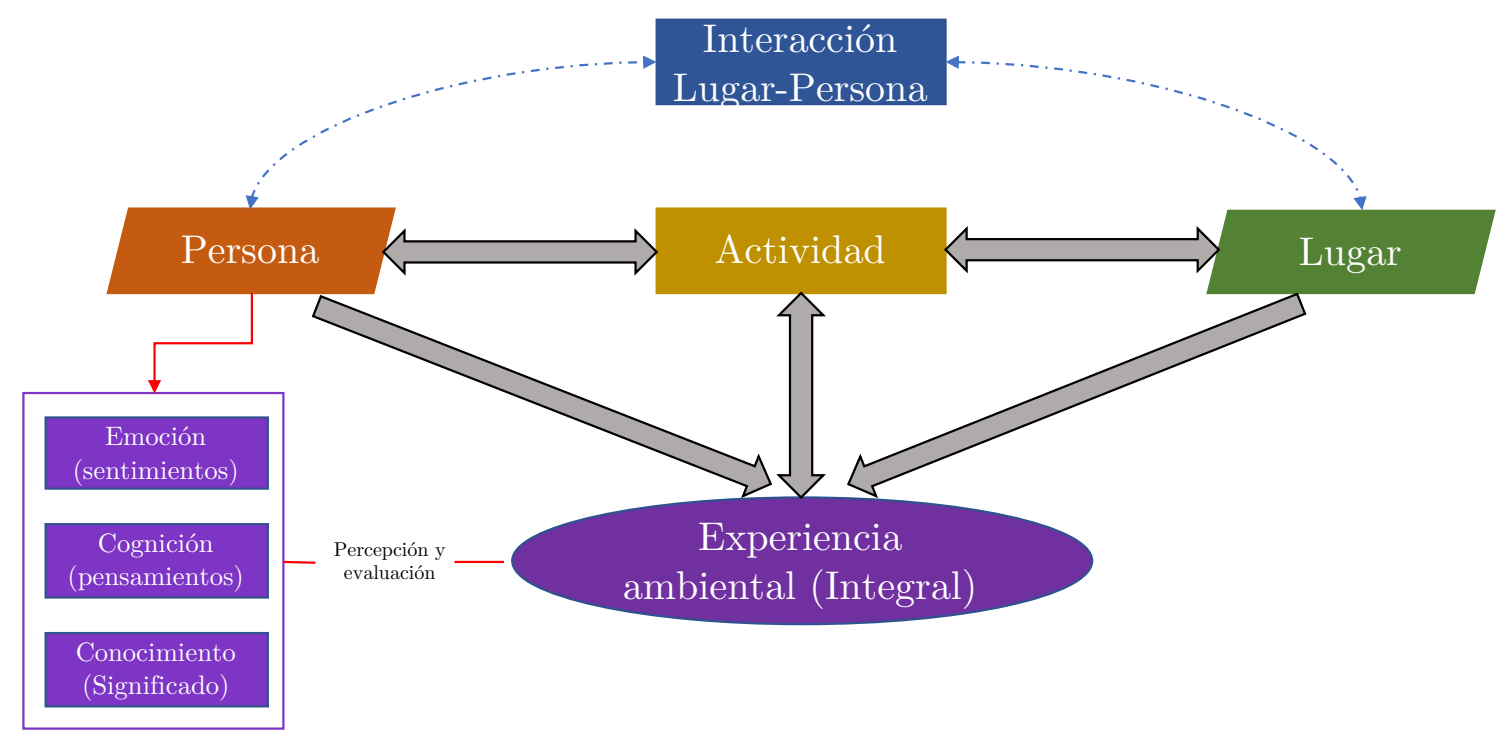

Figura 3-3. Modelo general conceptual de experiencia ambiental (Herranz-Pascual, 2010). Traducción propia.

El modelo general conceptual para experiencias ambientales se divide en tres grandes aspectos: persona, actividades y lugares. El aspecto de persona no solo se refiere a una persona sino a la comunidad, usuarios, etc. La relación entre persona y lugar es especial y tiene un carácter bidimensional por lo que un componente de experiencia previa es incluido en el modelo (interacción lugarpersona) diferenciando la actividad actual de las experiencias pasadas. La relación lugar-persona incluye experiencias previas, familiares, identidad de lugar, etc. 
Este modelo general permite analizar cualquier experiencia ambiental desde una mirada holística incluyendo diferentes aspectos psicosociales (emoción, cognición y significado).

\subsubsection{Modelo conceptual de experiencia ambiental en el estudio de paisajes sonoros.}

A partir del modelo general de experiencia ambiental presentado en la figura 3-3, Herranz-Pascual proponen el modelo de experiencia ambiental aplicado al estudio de paisajes sonoros $[7,16,17,21,22]$. Este modelo, como fue mencionado en el capítulo 1, está conformado por la triada Persona, Actividad y Lugar. A partir de estos tres aspectos se generan cuatro grupos que pueden influir en la experiencia del entorno acústico: Persona, Actividad, Lugar e Interacción lugarpersona (ver figura 1-5).

Según el modelo de Herranz-Pascual, cada lugar tiene su propio paisaje visual, entorno arquitectónico y atributos estéticos, acústicos y socio-culturales, los cuales varían según la hora, la estación o el clima. Así mismo, cuando una persona llega a un lugar, llega con su experiencia previa, la cual está mediada por hábitos, estilo de vida, preferencias, etc. La persona y el lugar interactúan a partir de las experiencias previas en ese lugar, la familiaridad e identidad y la información y expectativas que se tengan $[7,16]$. A partir de estos aspectos la persona podrá realizar actividades, las cuales según Herranz-Pascual pueden ser activas o pasivas.

Este es un modelo donde los diferentes componentes interactúan, por lo que cualquier cambio en alguno de ellos puede generar cambios en la construcción perceptual del paisaje sonoro.

Llevar el modelo de experiencia ambiental de paisajes sonoros a una práctica efectiva de evaluación de paisajes sonoros supone un gran esfuerzo de investigación. Es necesario determinar cuáles son los aspectos más influyentes en la experiencia ambiental, así como establecer procesos y procedimientos para este tipo de análisis. En la actualidad la mayoría de estudios en este campo se centran en la influencia de estímulos alternativos a los sonoros [21,23-29], detectando que uno de los estímulos más influyente en la percepción del entorno acústico se relaciona con aspectos visuales. Respecto al estudio de aspectos contextuales relacionados con la interacción lugar-persona, se tiene referencia de análisis de 
evaluaciones de paisajes sonoros en lugares de uso turístico, donde se comparan las evaluaciones entre turistas y no turistas, así como entre expertos y no expertos $[30,31]$. Sin embargo, hasta el momento no han sido especificadas diferencias entre salidas y respuestas variando aspectos culturales y de experiencia de los oyentes.

3.1.3 Calidad sonora y elementos de la semiótica en el estudio de paisajes sonoros.

Según Schulte y Fiebig, existen una gran variedad de trabajos que utilizan el término de calidad sonora (sound quality) para referirse al estudio de sonidos placenteros o afectivos, buscando de esta forma diferenciarse de trabajos que se enfocan en la molestia que generan los sonidos [5]. Los paisajes sonoros no han sido la excepción, buscando tomar distancia de trabajos de acústica ambiental enfocados en el concepto tradicional de molestia frente al ruido.

En lo que a aspectos conceptuales se refiere, a diferencia del idioma español, donde existen palabras diferentes para definir calidad y cualidad, en el inglés se tiene una única palabra para estos dos significados (quality) generado dos grandes vertientes respecto a la definición del término sound quality. Por una parte, se tienen autores que vinculan este término con las características cualitativas del sonido, las cuales son relacionadas con elementos perceptuales, indicando que sound quality se refiere a "un atributo distinguible, el atributo de una sensación elemental que hace que sea fundamentalmente diferente" sin establecer lo bueno o malo que es un sonido sino sus características específicas [32]. De otro lado diversos autores relacionan el concepto sound quality con la "reacción perceptual al sonido de un producto que refleja las reacciones del oyente a lo aceptable que el sonido del producto es", con "el grado en que la totalidad de los requerimientos individuales hechos de un evento auditivo son satisfechos" [33], o con "lo adecuado que es un sonido respecto a un producto" [34].

Buscando disipar la disputa conceptual en términos de calidad sonora, en la última década se han presentado dos enfoques. De un lado se propuso hacer la distinción entre carácter sonoro (sound character) y calidad sonora (sound quality), donde el carácter sonoro está relacionado con atributos básicos de un evento sonoro, sin considerar aspectos de contexto, actividades y/o altos niveles cognitivos, mientras que la calidad sonora describe la percepción adecuada del sonido afectada por factores como contexto, cognición e interacción [35,36]. De 
otro lado puede realizarse una diferenciación entre la característica del sonido y la calidad sonora a partir de diferentes niveles de abstracción, donde el nivel más básico (o primer nivel) se refiere a las propiedades del sonido, mientras que el más alto se refiere a aspectos elevados cognitivos relacionados con ideas y conceptos $[37]$.

El proceso de evaluación de calidad sonora se divide en dos partes: evaluación objetiva y evaluación subjetiva. En la evaluación objetiva se busca caracterizar el sonido en estudio a partir de parámetros acústicos y psicoacústicos, mientras que en la evaluación subjetiva se realiza un juicio por parte de oyentes (jurados) respecto a atributos del sonido. Por último, en las pruebas de evaluación de calidad sonora se busca determinar el grado de correlación existente entre aspectos objetivos y subjetivos [32]. Puede apreciarse, la gran similitud existente entre la evaluación de paisajes sonoros y la evaluación de la calidad sonora, más si se tiene en cuenta que las aplicaciones con mayor potencial se relacionan con el diseño sonoro y la gestión ambiental.

Por otra parte, según Blauert "el comportamiento de los seres humanos no está guiado por las señales acústicas que les proporcionamos, sino por el significado que se transfiere a través de ellas" [38], lo que implica que los sonidos llevan también una importante carga informativa al ser considerados como portadores de significados. Lo anterior implica tener que hacer un breve recorrido por algunos conceptos básicos de comunicación y semiótica a fin de usarlos en el estudio y evaluación de paisajes sonoros.

La semiótica es la ciencia que estudia los signos (y lenguajes), siendo el signo la representación mental que es procesada como referencia de otra cosa [3941]. El signo es un elemento de información (la envía y la recibe), por lo que se tienen muchos ejemplos de signos, como luces del semáforo, números o el habla. Sin embargo, para que exista la representación mental se requiere de un conocimiento previo de los objetos percibidos, así como de la relación entre el objeto primario de percepción y el concepto que este denota con el signo. En este sentido, para descifrar la información que porta un signo, el repertorio y el contexto juegan un papel fundamental. El estudio de los entornos urbanos bajo el enfoque semiótico ha permitido analizar el impacto de diferentes tipos de información (principalmente visual) en la identidad del lugar y el significado 
adquirido según el contexto de la persona [42-45]. Aplicando este enfoque en el estudio de paisajes sonoros resulta interesante plantearse la pregunta: ¿Cuál es el significado que los diseñadores, administradores y planificadores urbanos quieren transmitir a las personas desde entornos acústicos?

Según el enfoque semiótico, todos los sonidos pueden ser considerados como un portador de significado [33]. Los sonidos de las campanas de una iglesia, de los grillos que llegan anunciando el fin del verano o cualquier sonido producto de una actividad específica, transmiten información relacionada con el ambiente, así como con las prácticas o costumbres de una comunidad. En la semiosis ${ }^{2}$, los eventos perceptuales (ítems de percepción) son procesados en vista del conocimiento previo, de lo que la situación actual es, de lo que se anticipa a partir de cual sea la experiencia previa el oyente, quien finalmente asigna un significado a dicho signo. Dicho modelo es presentado en la figura 3-4.

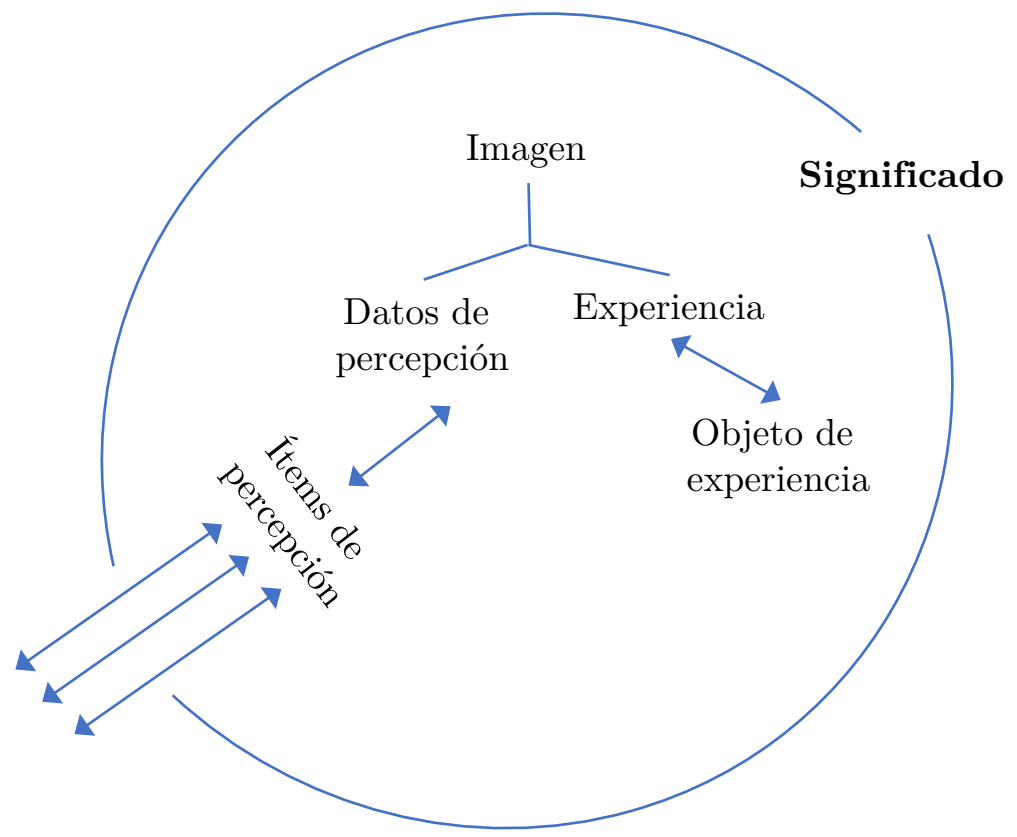

Figura 3-4. Modelo de semiosis de Jekosch (2005) (traducción propia).

Si bien los aspectos teóricos tratados en esta sección no pertenecen al campo de conocimiento de la ingeniería, se considera que estos son de gran importancia a fin de comprender resultados numéricos producto de las evaluaciones de entornos acústicos, así como de sus salidas y respuestas. Bajo el enfoque de paisajes sonoros, la caracterización de entornos acústicos no es

\footnotetext{
${ }^{2}$ Semiosis es un proceso de selección, organización, coordinación y estructuración, no solo de los ítems de percepción sino también de los objetos de experiencia [39].
} 
suficiente para la evaluación y gestión acústica ambiental, y como puede verse en la aproximación teórica presentada en esta sección, la percepción sonora no solo involucra aspectos relacionados con el estímulo que ofrece el entorno, sino también el contexto del oyente. Por lo tanto, teniendo en cuenta que en la actualidad se viene trabajando en procesos de estandarización en la evaluación de paisajes sonoros, se considera pertinente que los trabajos de investigación en este campo clarifiquen la influencia del contexto en las salidas y respuestas según el tipo de metodología usada.

\subsection{Aspectos metodológicos.}

Teniendo en cuenta el tercer objetivo específico propuesto para esta tesis doctoral, relacionado con el análisis de la influencia en las salidas y respuestas de paisajes sonoros por parte de la interacción lugar-persona, según conocimiento de lugares y de tipos de metodologías de evaluación, se desarrollaron dos pruebas de evaluación de paisajes sonoros. La primera prueba consiste en la comparación entre las respuestas de evaluadores in situ y en laboratorio, lo que permite apreciar la importancia de estímulos alternativos a los sonoros, así como las fortalezas, debilidades y posibles aplicaciones de este tipo de pruebas.

La segunda prueba busca determinar diferencias estadísticamente significativas en las respuestas y salidas de paisajes sonoros para evaluadores con diferentes contextos (interacción lugar-persona) y su impacto en procesos de diseño, gestión y planeación acústica. Para ello fueron desarrolladas pruebas pareadas en laboratorio en las ciudades de Lisboa (Portugal) y Bogotá (Colombia), presentándole a dos grupos de oyentes (lisboetas y bogotanos) entornos sonoros entornos acústicos tanto de su respectiva ciudad, como de la ciudad en donde no tienen experiencia previa. A continuación, se presenta una descripción detallada de las pruebas realizadas.

\subsubsection{Lugares en estudio.}

Para la evaluación de paisajes sonoros, además de los 5 lugares de la ciudad de Bogotá-Colombia descritos en el capítulo 2, fueron escogidos tres lugares de la ciudad de Lisboa (Portugal). Los lugares de Lisboa fueron parques urbanos de gran tradición entre los habitantes de Lisboa. Estos parques son: 
- Parque da Estrela (LJES),

- Jardín de la fundación Gulbenkian (LJFG)

- Jardín del Príncipe Real (LJPR).

Los parques tienen diferentes usos, características y ubicaciones, con áreas urbanas de 7,5ha para LJFG, 4,6ha para LJES y 1,15ha para LJPR (ver Anexo 5). Estos parques cuentan con fuentes de agua, vegetación diversa, espacios de juegos para niños, buena infraestructura, limpieza y seguridad y se encuentran en zonas privilegiadas de la ciudad, rodeados de edificios de fachadas alineadas con alturas máximas de seis pisos, donde el ruido de tráfico rodado y aéreo puede ser percibido por los habitantes.

Los lugares de la ciudad de Bogotá coinciden con los descritos en la sección 2.2.1, presentados en el Anexo 1:

- Autopista norte estación Alcalá (BOAPA).

- Calle 17 con carrera 7, centro de la ciudad (BOC197).

- Fuente de Agua edificio Tequendama (BOFAET).

- Plaza de Lourdes (BOPL).

- Parque Simón Bolívar (BOPSB).

\subsubsection{Pruebas in situ vs. Laboratorio.}

Para la comparación entre pruebas in situ y pruebas de laboratorio fueron evaluados tres parques urbanos de la ciudad de Lisboa, para lo cual se aplicaron pruebas in situ a los visitantes y se realizaron evaluaciones subjetivas en laboratorio (donde se presentaron muestras de entornos acústicos a un panel de oyentes quienes respondieron un cuestionario).

Fueron tomadas muestras de audio de los tres parques con el mismo sistema de grabación binaural descrito en la sección 2.2.1. Se trabajó en puntos fijos de escucha y grabación, escogidos según los lugares en que acostumbran a estar los visitantes. La prueba in situ fue a aplicada a personas que visitaban el parque, escogiéndolas de forma aleatoria (veinte por cada parque para un total de sesenta personas). La herramienta de aplicación in situ estaba compuesta por seis aspectos: un perfil demográfico, la relación con el parque, aspectos de infraestructura, impresión general, evaluación del entorno acústico y perfil sonoro del oyente (ver Anexo 6). El tipo de respuestas variaba según las preguntas, por 
lo que respuestas dicotómicas (si/no) o de escala de respuestas semántica de cinco pasos fueron usadas en la herramienta de aplicación in situ. Las respuestas de escala semántica ofrecían cinco niveles de respuesta: de acuerdo completamente, de acuerdo parcialmente, ni de acuerdo ni en desacuerdo, parcialmente en desacuerdo y totalmente en desacuerdo. Para el procesamiento de datos, cada uno de estos niveles tomaron valores entre +1 a un paso de 0.5 , siendo 1 concuerdo completamente y -1 totalmente en desacuerdo.

El número de evaluadores se ajustó a las recomendaciones dadas para trabajos de sound quality descrito en la sección 2.2.3 [46,47], por lo que la prueba de laboratorio fue aplicada a 25 oyentes (12 expertos y 13 no expertos), a quienes les fueron presentados fragmentos de grabaciones binaurales hechas en cada uno de los parques. Tanto expertos como no expertos conocían los tres parques en estudio. Las personas consideradas expertas tenían entrenamiento en el campo de acústica. Cada fragmento de audio tenía una duración de 30 segundos teniendo en consideración aspectos de edición según lo descrito en la sección 2.2.2. La grabación de los entornos acústicos fue presentada al panel de evaluadores por medio de auriculares. Tanto la grabación como la reproducción fue calibrada usando un torso artificial de marca 01dB a fin de asegurar que en las pruebas de laboratorio fueran presentados niveles próximos a los existentes en los entornos acústicos in situ. El proceso de calibración corresponde al presentado en la figura 2-8. Las pruebas de laboratorio fueron desarrolladas en la cámara anecóica del Instituto Superior Técnico (IST) de la Universidad de Lisboa. Al igual que en las pruebas subjetivas del capítulo 2, la herramienta de evaluación en laboratorio está dividida en cuatro grandes secciones: perfil general del oyente, aspectos descriptivos (evaluación de sonido fundamental y marca sonora) respuestas a corto plazo (atributos perceptuales según modelo de Axelsson) y salidas a largo plazo (información relacionada con el uso y el tiempo de permanencia) tal como se presenta en la figura 2-9 y el Anexo 7 (herramienta traducida al portugués).

3.2.3 Pruebas según contexto del oyente: respuestas y salidas en evaluaciones de paisajes sonoros Lisboa vs. Bogotá.

En esta prueba fueron evaluados los tres paisajes sonoros de la ciudad de Lisboa y los cinco paisajes sonoros de Bogotá para un total de ocho paisajes sonoros. Las pruebas fueron aplicadas en laboratorio, presentando a los oyentes 
exclusivamente los estímulos sonoros a partir de grabaciones binaurales de treinta segundos, lo que permite descartar cualquier otro tipo de estímulo (visual u olfativo) que pueda afectar en los resultados de la evaluación. La herramienta de aplicación corresponde a la misma herramienta aplicada en las pasadas pruebas de laboratorio presentadas en los anexos 4 y 7 .

A diferencia del capítulo 2, donde se analizó la correlación entre parámetros objetivos y atributos perceptuales (respuestas a corto plazo), en las pruebas comparativas entre oyentes de Bogotá y Lisboa, además de tener en cuenta los atributos perceptuales según el modelo de Axelsson (relacionado con respuestas o actitudes de los oyentes), se profundizó en el estudio de tiempo de permanencia y uso que darían los oyentes a un lugar con un entorno acústico como el presentado en el audio (salidas o comportamientos). Los tiempos de permanencia se presentaron por rangos (menos de 10 minutos, entre 10 y 30 minutos, entre 30 y 60 minutos, entre 1 hora y dos horas y más de dos horas), mientras que los posibles usos que los oyentes darían a un entorno acústico se presentaron a partir de un listado de actividades (actividad física, lugar de tránsito, contemplación, lectura y meditación, descanso general trabajo y otro).

Con el fin de obtener información descriptiva de los entornos acústicos relacionada con aspectos descriptivos, para identificar los sonidos fundamentales de los ocho lugares, la prueba subjetiva incluyó una lista de sonidos y se pidió a los participantes que indicaran cuan dominantes percibían cada uno de ellos. Al igual que las pruebas descritas en el capítulo 2, sección 2.2.3, las opciones de respuesta fueron "no dominante", "poco dominante", "moderadamente dominante", "muy dominante" y "totalmente dominante" y cada opción corresponde a valores de $-1,-0.5,0,0.5$ y 1 respectivamente. Así mismo, si bien el objetivo de este trabajo se centra en profundizar el estudio de los aspectos contextuales y semióticos y su relación con los procesos de gestión y planificación de paisajes sonoros urbanos, se consideró relevante incluir un indicador acústico tradicional para complementar los aspectos descriptivos de los entornos acústicos. En este estudio, se usó el nivel equivalente de presión de sonido ponderado por A LAeq.

El proceso de calibración del sistema de grabación y reproducción corresponde al presentado en la figura 2-8. En la evaluación de los parques de 
Lisboa participaron un total de 75 personas (25 en Lisboa y 50 en Bogotá) mientras que para la evaluación de los lugares de Bogotá participaron 50 personas (25 en Lisboa y 25 en Bogotá). Cada oyente evaluó 8 fragmentos de 30 segundos de audio correspondientes a los 3 parques de Lisboa y los 5 lugares de Bogotá. El tiempo total de la prueba establecido era de máximo 20 minutos para cada oyente según las consideraciones de duración máxima recomendada en las pruebas de calidad sonora presentadas en la sección $2.3[32,47,48]$. Las pruebas de laboratorio fueron desarrolladas en la cámara anecóica del Instituto Superior Técnico (IST) de la Universidad de Lisboa y en los estudios de grabación de la Universidad de San Buenaventura sede Bogotá (que cumple con una curva NC 25 según lo descrito en la sección 2.2.3.

La figura 3.5 presenta un resumen de las pruebas desarrolladas en el análisis de contexto desarrollado en este capítulo.

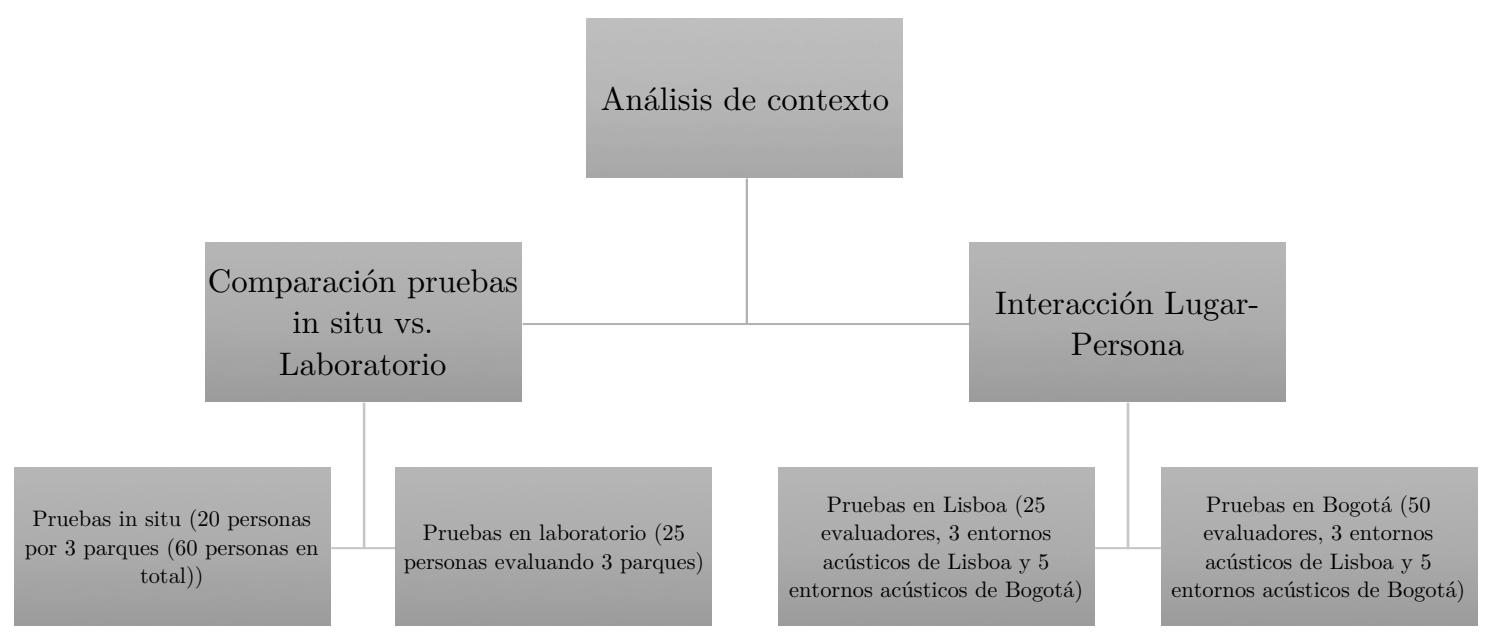

Figura 3-5. Resumen pruebas de análisis de contexto.

\subsubsection{Tratamiento estadístico de datos.}

Para todos los datos fueron obtenidas medidas de tendencia central y medidas de dispersión. Fue aplicada la prueba de normalidad Shapiro-Wilk para determinar el tipo de prueba que debía realizarse (paramétrica o no paramétrica) y detectar diferencias estadísticamente significativas entre las metodologías de evaluación (in situ y laboratorio), expertos y no expertos y entre oyentes de Lisboa y Bogotá. Dado que los datos no presentaban distribuciones normales fueron aplicadas pruebas no paramétricas. 
Para la comparación entre evaluaciones in situ y en laboratorio y, expertos y no expertos fue aplicada la prueba U de Mann-Whitney. Dicha prueba es de tipo no paramétrica y es usada para la comparación de dos muestras independientes, que está basado en la comparación de los rankings alcanzados con el mejor puntaje posible [49]

En el estudio comparativo entre diferencias de respuestas a corto plazo y salidas a largo plazo entre oyentes de Lisboa y Bogotá, los datos fueron analizados a partir de pruebas de U de Mann-Whitney para los datos numéricos (respuestas a corto plazo producto de la evaluación de atributos perceptuales) y tablas de contingencia para datos nominales (en el caso de las preguntas relacionadas con el tiempo en el que permanecería en un entorno acústico como ese y el uso que daría a ese espacio), donde fueron aplicadas las pruebas de significancia estadística Chi-Cuadrado de Pearson y la Razón de verosimilitud de Chi-cuadrado.

\subsection{Análisis de resultados.}

3.3.1 Comparación pruebas in situ vs. laboratorios.

Las figuras 3-6 y 3-7 y la tabla 3-1 presentan las medidas de tendencia central (media y mediana), las distribuciones de los atributos perceptuales y la evaluación general de los paisajes sonoros para los tres parques de Lisboa. A primera vista parecieran existir diferencias entre las pruebas in situ y en laboratorio en atributos como Caótico (más caótico en laboratorio), Agradable (más agradable in situ), Uniforme/Sin eventos (más uniforme in situ) y Calmo (más calmo in situ). Sin embargo, para determinar si las diferencias entre las respuestas según el tipo de prueba aplicada son estadísticamente significativas, deben aplicarse las pruebas de Mann-Whitney para evaluaciones no paramétricas. 


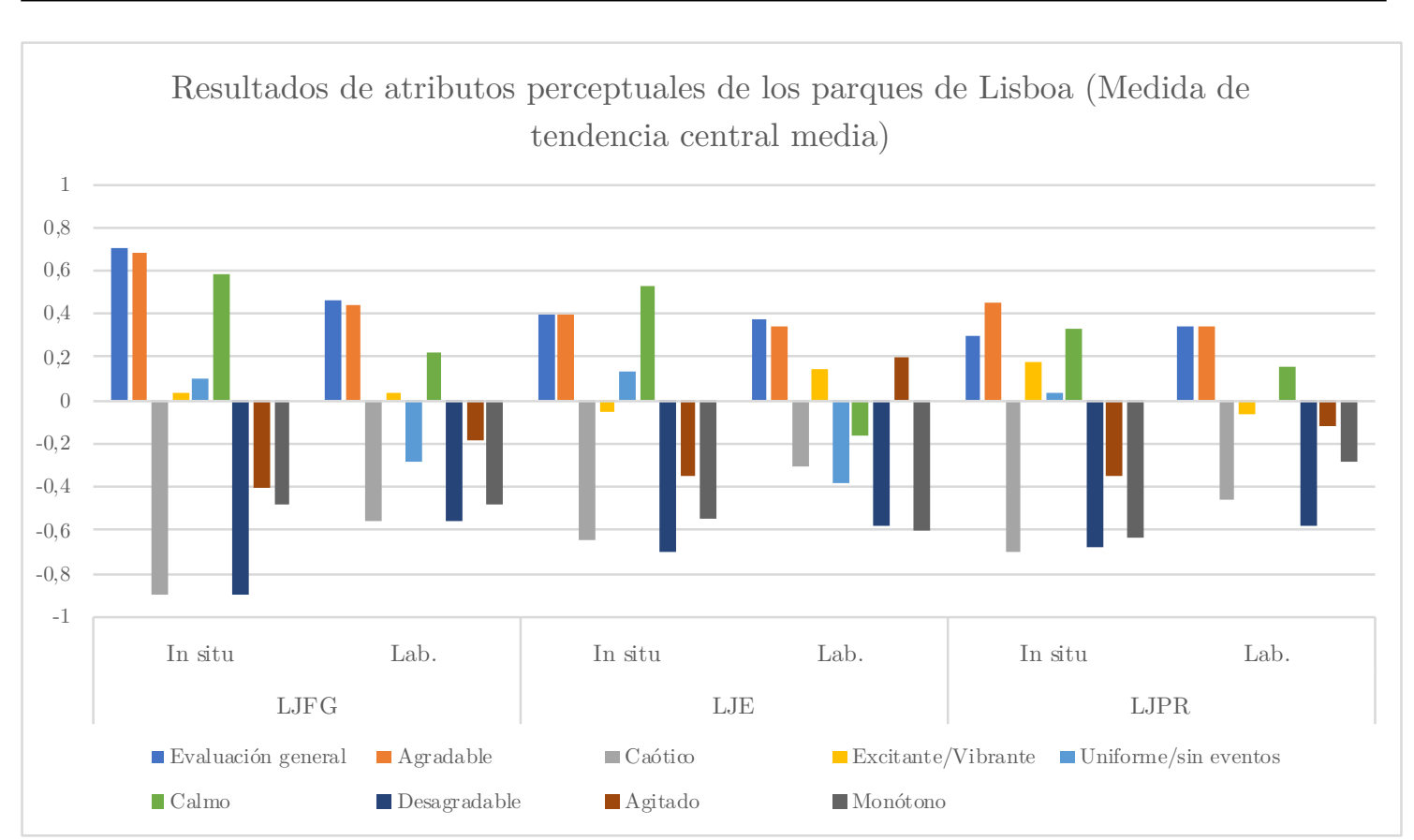

Figura 3-6. Medias de atributos perceptuales (respuestas a corto plazo) en pruebas in situ y en laboratorio para parques de Lisboa-Portugal.

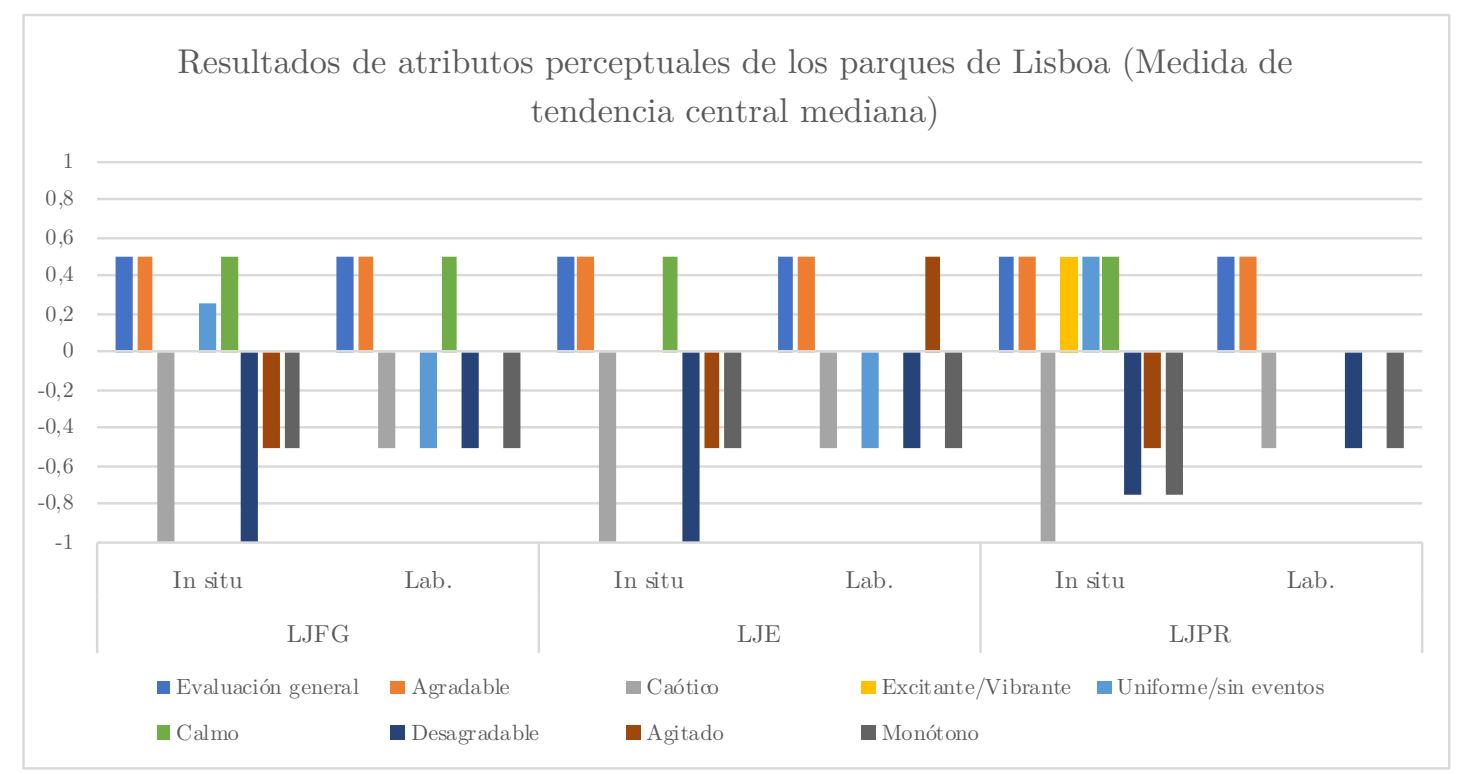

Figura 3-7. Medianas de atributos perceptuales (respuestas a corto plazo) en pruebas in situ y en laboratorio para parques de Lisboa-Portugal.

Así mismo, la tabla 3-1 permite apreciar en dos de los tres parques se presentaron mayores desviaciones estándar en las pruebas in situ que en laboratorio, lo que puede deberse a que en las pruebas en laboratorio siempre se presentaban los mismos 30 segundos de grabación para cada parque, mientras que en las pruebas in situ el entorno acústico se renovaba continuamente. 
Tabla 3-16. Desviaciones estándar de los tres parques en Lisboa-Portugal para las pruebas in situ vs. laboratorio. Casillas en sombra gris presentan menores valores de desviaciones en las pruebas de laboratorio.

\begin{tabular}{|c|c|c|c|c|c|c|c|c|c|c|}
\hline Lugar & Prueba & $\begin{array}{c}\text { Eval. } \\
\text { general }\end{array}$ & Agradable & Caótico & $\begin{array}{c}\text { Excitante } \\
\text { /Vibrante }\end{array}$ & $\begin{array}{c}\text { Uniforme/ } \\
\text { sin eventos }\end{array}$ & Calmo & Desagradable & Agitado & Monótono \\
\hline \multirow{2}{*}{ LJFG } & In situ & 0,30 & 0,24 & 0,31 & 0,38 & 0,48 & 0,37 & 0,26 & 0,50 & 0,60 \\
\cline { 2 - 12 } & Lab. & 0,35 & 0,49 & 0,42 & 0,41 & 0,48 & 0,50 & 0,42 & 0,45 & 0,27 \\
\hline \multirow{2}{*}{ LJE } & In situ & 0,42 & 0,55 & 0,54 & 0,39 & 0,48 & 0,60 & 0,64 & 0,54 & 0,48 \\
\cline { 2 - 12 } & Lab. & 0,26 & 0,35 & 0,56 & 0,51 & 0,51 & 0,43 & 0,31 & 0,41 & 0,29 \\
\hline \multirow{2}{*}{ LJPR } & In situ & 0,30 & 0,36 & 0,38 & 0,47 & 0,60 & 0,37 & 0,47 & 0,49 & 0,46 \\
\cline { 2 - 12 } & Lab. & 0,28 & 0,40 & 0,38 & 0,44 & 0,50 & 0,37 & 0,34 & 0,48 & 0,38 \\
\hline
\end{tabular}

\subsubsection{Diferencias de atributos perceptuales según metodología de evaluación:} in situ vs. laboratorio.

Dado que los resultados obtenidos no presentaban una distribución normal (Anexo 8), fueron aplicadas pruebas no paramétricas para detectar diferencias estadísticamente significativas en los atributos perceptuales según la metodología usada. La tabla 3-2 presenta los resultados obtenidos con las pruebas no paramétricas de Mann-Whitney.

Fueron encontradas diferencias estadísticamente significativas entre las pruebas in situ y laboratorio a un nivel de significancia del 5\%, aunque los atributos que presentaron estas diferencias varían según el lugar. De forma específica, en LJFG fueron encontradas diferencias en la evaluación general, así como en los atributos Caótico, Uniforme, Calmo y Molesto. En las pruebas in situ este lugar tuvo una mejor evaluación general y fue considerado más calmo y sin eventos, mientras que en laboratorio fue considerado más Caótico y Molesto. En el LJE se encontraron diferencias estadísticamente significativas en los atributos Caótico, Uniforme/sin eventos, Calmo, Molesto y Agitado. Este lugar fue percibido como más Caótico, Molesto y Agitado en laboratorio, mientras que fue encontrado más Uniforme y Calmo in situ. El parque LPR presenta diferencias estadísticamente significativas en los atributos Caótico y Monótono, mostrando mayores valores en estos dos atributos en las pruebas de laboratorio. 
Tercer Capítulo. Paisajes sonoros: Respuestas y Salidas y su relación con el contexto

Tabla 3-17. Resultados de las pruebas no paramétricas de Mann-Whitney para parques de Lisboa. Metodologías In situ. vs. Laboratorio. Resaltadas en verde se presentan los $p$ valores que presentan diferencias estadísticamente significativas.

\begin{tabular}{|c|c|c|c|c|c|c|c|c|c|c|}
\hline Lugar & Estadístico & $\begin{array}{c}\text { Evaluación } \\
\text { general }\end{array}$ & Agradable & Caótico & Excitante/Vibrante & Uniforme/sin eventos & Calmo & Desagradable & Agitado & Monótono \\
\hline \multirow{4}{*}{ LJFG } & Mann-Whitney U & 160 & 183,5 & 125 & 243,5 & 145,5 & 145,5 & 127,5 & 178 & 226,5 \\
\hline & Wilcoxon W & 485 & 508,5 & 335 & 453,5 & 470,5 & 470,5 & 337,5 & 388 & 436,5 \\
\hline & $\mathrm{Z}$ & $-2,320$ & $-1,710$ & $-3,250$ & $-0,160$ & $-2,510$ & $-2,70$ & $-3,164$ & $-1,766$ & $-0,580$ \\
\hline & Asymp. Sig. (2-tailed) & 0,020 & 0,080 & 0,001 & 0,870 & 0,012 & 0,007 & 0,002 & 0,077 & 0,562 \\
\hline \multirow{4}{*}{ LJE } & Mann-Whitney U & 212 & 206 & 153 & 186 & 120,5 & 80,5 & 155 & 107,5 & 248,5 \\
\hline & Wilcoxon W & 537 & 531 & 363 & 396 & 445,5 & 405,5 & 365 & 317,5 & 573,5 \\
\hline & $\mathrm{Z}$ & $-1,150$ & $-1,220$ & $-2,320$ & $-1,560$ & $-3,06$ & $-3,989$ & $-2,383$ & $-3,425$ & $-0,037$ \\
\hline & Asymp. Sig. (2-tailed) & 0,240 & 0,210 & 0,020 & 0,110 & 0,002 & 0,000 & 0,017 & 0,001 & 0,970 \\
\hline \multirow{4}{*}{ LJPR } & Mann-Whitney U & 241,50 & 204,5 & 164 & 181 & 233,5 & 190 & 195,5 & 185,5 & 135,5 \\
\hline & Wilcoxon W & 451,50 & 529,50 & 374 & 506 & 558,5 & 515 & 405,5 & 395,5 & 345,5 \\
\hline & $\mathrm{Z}$ & $-0,220$ & $-1,130$ & $-2,110$ & $-1,660$ & $-0,390$ & $-1,530$ & $-1,376$ & $-1,539$ & $-2,789$ \\
\hline & Asymp. Sig. (2-tailed) & 0,810 & 0,250 & 0,034 & 0,096 & 0,691 & 0,126 & 0,169 & 0,124 & 0,005 \\
\hline
\end{tabular}


De forma general los resultados permiten apreciar que las pruebas aplicadas en laboratorio eran evaluadas como más Caóticas, esto para los tres lugares en estudio. Se puede ver que los lugares eran percibidos más Uniformes en las pruebas in situ que en laboratorio, por lo que de forma complementaria fueron evaluados más Agitados en laboratorio que in situ. Estos resultados muestran la importancia que tiene el estímulo sonoro respecto a la información temporal: evanescente y cambiante en el tiempo como fue descrito por Lucia Santaella [40]. Así mismo muestra que, en estos casos de estudio aplicados a parques urbanos, otro tipo de estímulos son de gran importancia en la configuración de las respuestas de los oyentes. Un claro ejemplo de esto último es el LJFG, lugar proyectado con especial cuidado en aspectos visuales y olfativos y donde diferencias estadísticamente significativas en la evaluación general y el atributo Molesto permiten apreciar la importancia de estímulos alternos a los sonoros.

\subsubsection{Componentes principales de Eventfulness y Pleasantness en parques urbanos de Lisboa.}

A partir de los ocho atributos perceptuales, fueron calculados los componentes principales en el sistema de coordenadas cartesianas según el modelo de Axelsson según las expresiones 4 y 5 del capítulo 2 (Tabla 3-3). Debido a la falta de normalidad de las diferentes variables, tanto en las evaluaciones in situ como en laboratorio, los cálculos fueron realizados teniendo en cuenta tanto medias como medianas. Puede apreciarse que en las pruebas in situ el componente principal de Pleasantness era mayor que en las pruebas in situ para los tres lugares en estudio. Así mismo, el componente Eventfulness presentó menores valores en las pruebas in situ que en laboratorio. Las evaluaciones generales de los entornos sonoros fueron más altas en las pruebas in situ que en las pruebas de laboratorio.

Tabla 3-18. Resultados de componentes principales según model de evaluación sueco.

\begin{tabular}{|c|c|c|c|c|c|c|c|}
\hline \multirow[b]{2}{*}{ Local } & \multirow{2}{*}{$\begin{array}{c}\text { Medida } \\
\text { de } \\
\text { tendencia } \\
\text { central }\end{array}$} & \multicolumn{3}{|c|}{ In situ } & \multicolumn{3}{|c|}{ Lab } \\
\hline & & Pleasantness & Eventfulness & $\begin{array}{l}\text { Evaluación } \\
\text { general }\end{array}$ & Pleasantness & Eventfulness & $\begin{array}{c}\text { Evaluación } \\
\text { general }\end{array}$ \\
\hline \multirow{2}{*}{ LJFG } & Media & 0.56 & -0.25 & 0.70 & 0.36 & -0.02 & 0.46 \\
\hline & Mediana & 0.54 & -0.30 & 0.50 & 0.40 & 0.03 & 0.50 \\
\hline \multirow{2}{*}{ LJE } & Media & 0.43 & -0.20 & 0.40 & 0.28 & 0.21 & 0.38 \\
\hline & Mediana & 0.54 & -0.25 & 0.50 & 0.32 & 0.21 & 0.50 \\
\hline \multirow{2}{*}{ LJPR } & Media & 0.46 & -0.11 & 0.30 & 0.28 & -0.08 & 0.34 \\
\hline & Mediana & 0.62 & -0.24 & 0.50 & 0.32 & 0.00 & 0.50 \\
\hline
\end{tabular}


Las figuras 3-8 y 3-9 presentan con mayor claridad los resultados obtenidos. Pueden apreciarse en las dos figuras dos grupos diferenciados entre las pruebas in situ y en laboratorio. Los resultados de los tres lugares en las pruebas in situ se encuentran en el cuadrante inferior derecho, que indica placer y poca cantidad de eventos. Por otra parte, los resultados de las pruebas en laboratorio presentan un desplazamiento hacia el cuadrante superior derecho, más claramente existente en la figura 3-8 (análisis con medianas) que en la figura 3-9.

El lugar que presenta las mayores variaciones representadas en el modelo bidimensional de Axelsson es LJE seguido por LJFG, mostrando los mayores desplazamientos del cuadrante inferior derecho (in situ) al cuadrante superior derecho (en laboratorio).

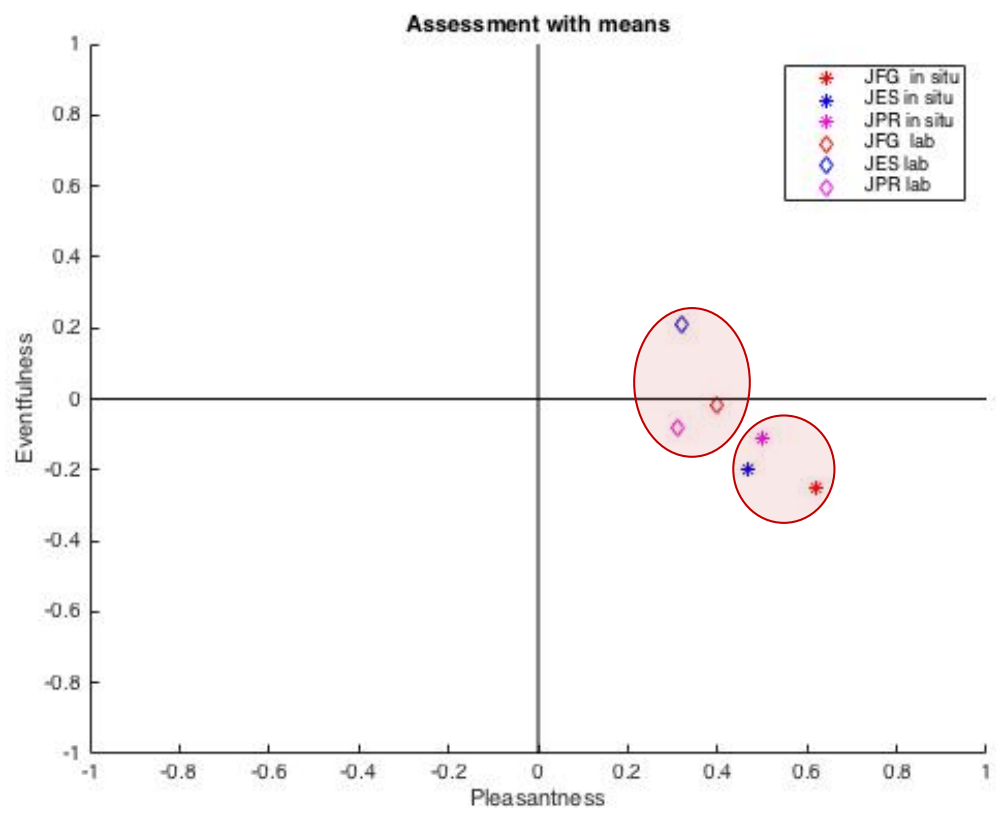

Figura 3-8 Representación gráfica de la evaluación de parques urbanos de la ciudad de Lisboa, usando como medida de tendencia central las medias. 


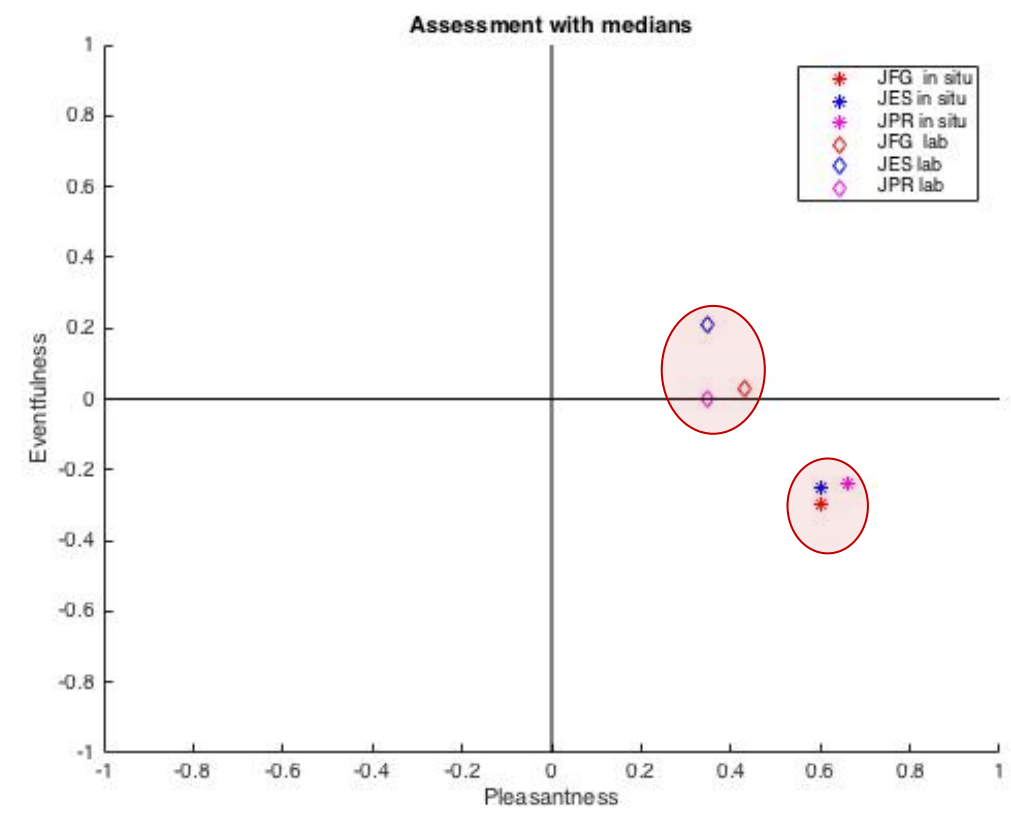

Figura 3-9 Representación gráfica de la evaluación de parques urbanos de la ciudad de Lisboa, usando como medida de tendencia central las medianas.

\subsubsection{Comparación de resultados entre oyentes expertos y no expertos para parques de Lisboa en pruebas de laboratorio.}

La tabla 3-4 presenta los resultados de las pruebas Mann-Whitney aplicadas para analizar las diferencias entre expertos y no expertos en las pruebas de laboratorio. Teniendo un nivel de significancia del 5\%, los resultados muestran que solo fue detectada diferencia estadísticamente significativa entre expertos y no expertos en el atributo Monótono. Esto quiere decir que para las pruebas aplicadas en los tres casos de estudio de parques de Lisboa no existen diferencias considerables en las evaluaciones entre expertos y no expertos.

Tabla 3-19. Resultados de pruebas de Mann-Whitney entre expertos y no expertos para parques de Lisboa (pruebas en laboratorio).

\begin{tabular}{|c|c|c|c|c|c|c|c|c|c|c|}
\hline Lugar & Estadístico & $\begin{array}{c}\text { Eval. } \\
\text { general }\end{array}$ & Agradable & Caótico & $\begin{array}{c}\text { Excitante } \\
\text { /Vibrante }\end{array}$ & $\begin{array}{c}\text { Uniforme } \\
/ \text { sin } \\
\text { eventos }\end{array}$ & Calmo & Desagradable & Agitado & Monótono \\
\hline \multirow{2}{*}{ LJFG } & $\begin{array}{c}\text { Mann- } \\
\text { Whitney U }\end{array}$ & 62 & 58 & 66.5 & 54 & 71 & 45.5 & 69.5 & 61.5 & 72.5 \\
\cline { 2 - 11 } & $\begin{array}{c}\text { Exact Sig. } \\
{[2 *(1-\text {-tailed }} \\
\text { Sig.) }\end{array}$
\end{tabular}




\begin{tabular}{|c|c|c|c|c|c|c|c|c|c|c|}
\multirow{2}{*}{ LJPR } & $\begin{array}{c}\text { Mann- } \\
\text { Whitney U }\end{array}$ & 46 & 55 & 72 & 51.5 & 63 & 63 & 60 & 71 & 74.5 \\
\cline { 2 - 9 } & $\begin{array}{c}\text { Exact Sig. } \\
{\left[\begin{array}{c}2 * \text { (1-tailed } \\
\text { Sig.) }\end{array}\right.}\end{array}$ & $.087 \mathrm{~b}$ & $.225 \mathrm{~b}$ & $.769 \mathrm{~b}$ & $.152 \mathrm{~b}$ & $.437 \mathrm{~b}$ & $\begin{array}{c}.437 \\
\mathrm{~b}\end{array}$ & $.347 \mathrm{~b}$ & $.728 \mathrm{~b}$ & $.852 \mathrm{~b}$ \\
\hline
\end{tabular}

b Not corrected for ties.

3.3.2 Evaluación en laboratorio de paisajes sonoros considerando el elemento interacción lugar-persona: comparación de evaluación de paisajes sonoros entre oyentes de las ciudades de Lisboa (Portugal) y Bogotá (Colombia).

Esta sección presenta la comparación entre los resultados de las evaluaciones realizadas por oyentes conocedores y no conocedores de los ocho paisajes sonoros de las ciudades de Lisboa-Portugal y Bogotá-Colombia. Las comparaciones son discriminadas entre respuestas a corto plazo (atributos perceptuales) y salidas a largo plazo (tiempos de permanencia y posible uso del lugar).

\subsubsection{Aspectos descriptivos de los entornos acústicos en estudios.}

La Tabla 3-5 presenta información sobre los sonidos fundamentales y el LAeq de los diferentes lugares en estudio. En relación a los sonidos fundamentales de los ocho lugares, tanto los evaluadores de Lisboa como los de Bogotá determinaron que el sonido fundamental es el tráfico vehicular para los lugares BOAPA y BOC197, para LJFG y BOPSB el sonido fundamental proviene de la naturaleza, la voz humana es el sonido fundamental para LJES y LJPR, y la fuente de agua es el sonido fundamental para El BOFAET. Solo BOPL presentó diferencias en los sonidos de apertura entre los evaluadores de Bogotá y Lisboa, donde el panel de Bogotá consideró que el sonido de apertura de este espacio es el discurso, mientras que el panel de Lisboa consideró que el sonido de apertura de este espacio es el tráfico rodado.

Respecto a los niveles de presión sonora, la Tabla 3-5 muestra que aquellos con niveles más altos corresponden a BOFAET (75.4 dBA) y BOC197 (74.6 dB), mientras que los niveles más bajos son de BOPSB (51.4 dBA) y LJPR (56.6 dBA). Los resultados permiten apreciar que los niveles más altos no se corresponden solo a los lugares con sonido fundamental del tráfico vehicular, así como los lugares 
con el LAeq más bajo no se corresponden únicamente a aquellos con el sonido fundamental de la naturaleza.

Tabla 3-20. Sonido Fundamental y nivel continuo equivalente ponderado A para los 8 paisajes sonoros en estudio.

\begin{tabular}{|c|c|c|c|}
\hline Lugar & $\begin{array}{l}\text { Ciudad de } \\
\text { evaluación }\end{array}$ & Sonido fundamental & LAeq (dBA) \\
\hline \multirow{2}{*}{ LJES } & Bogotá & Voz (valor medio 0.57 y $88 \%$ de detección) & \multirow{2}{*}{65.6} \\
\hline & Lisboa & Voz (valor medio 0.54 y $84 \%$ de detección) & \\
\hline \multirow{2}{*}{ LJFG } & Bogotá & Naturaleza (valor medio 0.43 y $80 \%$ de detección) & \multirow{2}{*}{58.4} \\
\hline & Lisboa & Naturaleza (valor medio 0.46 y $76 \%$ de detección) & \\
\hline \multirow{2}{*}{ LJPR } & Bogotá & Voz (valor medio 0.49 y $78 \%$ de detección) & \multirow{2}{*}{56.6} \\
\hline & Lisboa & Voz (valor medio 0.38 y $60 \%$ de detección) & \\
\hline \multirow{2}{*}{ BOAPA } & Bogotá & $\begin{array}{l}\text { Tráfico vehicular (valor medio } 0.66 \text { y } 96 \% \text { de } \\
\text { detección) }\end{array}$ & \multirow{2}{*}{63.9} \\
\hline & Lisboa & $\begin{array}{c}\text { Tráfico vehicular (valor medio } 0.70 \text { y } 96 \% \text { de } \\
\text { detección) }\end{array}$ & \\
\hline \multirow{2}{*}{ BOC197 } & Bogotá & $\begin{array}{l}\text { Tráfico vehicular (valor medio } 0.94 \text { y } 100 \% \text { de } \\
\text { detección) }\end{array}$ & \multirow{2}{*}{74.6} \\
\hline & Lisboa & $\begin{array}{l}\text { Tráfico vehicular (valor medio } 0.94 \text { y } 100 \% \text { de } \\
\text { detección) }\end{array}$ & \\
\hline \multirow{2}{*}{ BOFAET } & Bogotá & Agua (valor medio 0.80 y $93 \%$ de detección) & \multirow{2}{*}{75.4} \\
\hline & Lisboa & Agua (valor medio 0.90 y $100 \%$ de detección) & \\
\hline \multirow[b]{2}{*}{ BOPL } & Bogotá & Voz (valor medio 0.56 y $88 \%$ de detección) & \multirow[b]{2}{*}{68.7} \\
\hline & Lisboa & $\begin{array}{c}\text { Tráfico vehicular (valor medio } 0.50 \text { y } 92 \% \text { de } \\
\text { detección) }\end{array}$ & \\
\hline \multirow{2}{*}{ BOPSB } & Bogotá & Naturaleza (valor medio 0.50 y $80 \%$ de detección) & \multirow{2}{*}{51.4} \\
\hline & Lisboa & Naturaleza (valor medio 0.42 y $68 \%$ de detección) & \\
\hline
\end{tabular}

\subsubsection{Evaluación de respuestas a corto plazo. Evaluadores de Bogotá vs. Evaluadores de Lisboa.}

Las figuras 3-10a y 3-10b presentan los resultados obtenidos para los 8 atributos del modelo de Axelsson, así como para la evaluación general. Se pueden apreciar diferencias según el lugar de evaluación de los oyentes para atributos como Excitante, Monótono, Agitado, Desagradable entre otros, aunque para determinar si dichas diferencias son estadísticamente significativas es necesario aplicar de nuevo la prueba no paramétrica de U de Mann-Withney. La tabla 3-5 presenta los resultados de dicha prueba.

Para el lugar LJFG solo fueron encontradas diferencias estadísticamente significativas en los atributos Excitante y Monótono, donde según los valores de 
Mean Rank los evaluadores de Lisboa lo consideraban más excitante mientras que los evaluadores de Colombia lo calificaron más Monótono. El lugar LPE no presentó diferencias estadísticamente significativas, mientras que el lugar LPR presentó diferencias estadísticamente significativas solamente en el atributo Monótono (más monótono en Bogotá que en Lisboa).

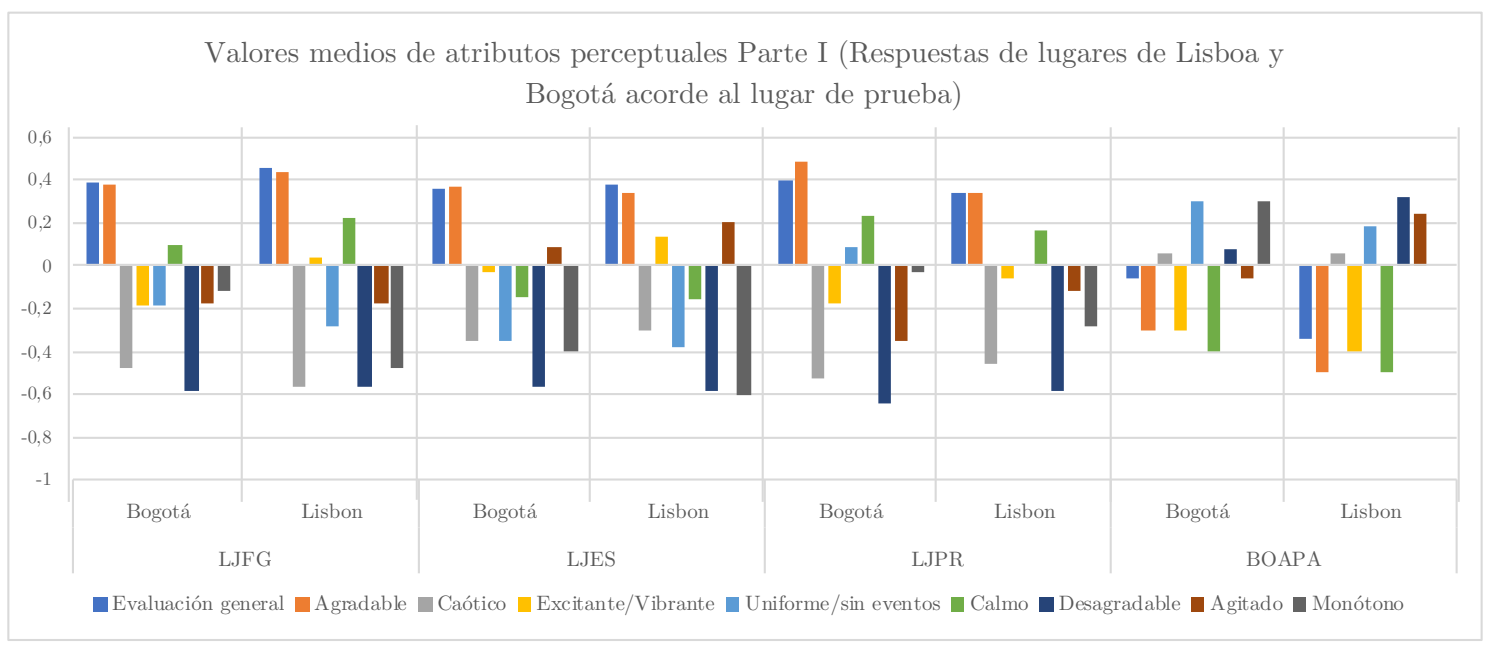

Figura 3-10. Valores medios de atributos perceptuales Parte I (respuestas a corto plazo de lugares de Lisboa y Bogotá según el lugar de prueba)

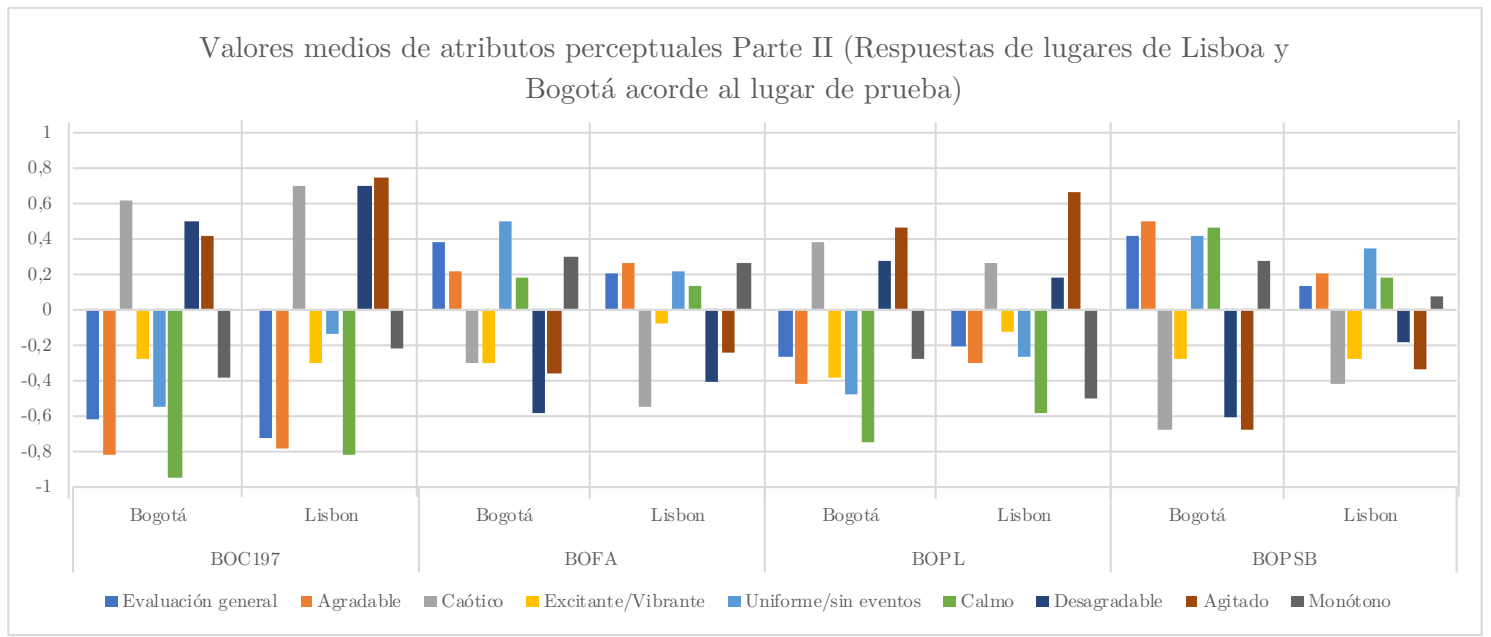

Figura 3-11. Valores medios de atributos perceptuales Parte II (respuestas a corto plazo de lugares de Lisboa y Bogotá según el lugar de evaluación) 
Tercer Capítulo. Paisajes sonoros: Respuestas y Salidas y su relación con el contexto

Tabla 3-21. Resultados de las pruebas de U Mann-Whitney entre evaluadores de Bogotá y evaluadores de Lisboa. Resaltadas en verde los p valores que indican diferencias estadísticamente significativas. Con sombra gris el mayor valor de rango promedio entre los oyentes de Bogotá y Lisboa para cada diferencia significativa.

\begin{tabular}{|c|c|c|c|c|c|c|c|c|c|c|}
\hline Lugar & & $\begin{array}{c}\text { Eval. } \\
\text { general }\end{array}$ & Agradable & Caótico & $\begin{array}{c}\text { Excitante/ } \\
\text { Vibrante }\end{array}$ & $\begin{array}{c}\text { Uniforme/ sin } \\
\text { eventos }\end{array}$ & Calmo & Desagradable & Agitado & Monótono \\
\hline \multirow{4}{*}{ LJFG } & Mann-Whitney U & 540.000 & 537.000 & 577.500 & 437.500 & 577.500 & 535.500 & 620.000 & 625.000 & 338.000 \\
\hline & Asymp. Sig. (2-tailed) & 0.282 & 0.286 & 0.571 & 0.024 & 0.575 & 0.281 & 0.952 & 1.000 & 0.001 \\
\hline & Mean Rank Bogotá & 36.300 & 36.240 & 38.950 & 34.250 & 38.950 & 36.210 & 37.900 & 38.000 & 43.740 \\
\hline & Mean Rank Lisboa & 41.400 & 41.520 & 36.100 & 45.500 & 36.100 & 41.580 & 38.200 & 38.000 & 26.520 \\
\hline \multirow{4}{*}{ LJPE } & Mann-Whitney U & 597.000 & 624.500 & 594.500 & 497.000 & 612.500 & 615.000 & 622.500 & 570.000 & 499.500 \\
\hline & Asymp. Sig. (2-tailed) & 0.731 & 0.995 & 0.722 & 0.126 & 0.882 & \begin{tabular}{|l|}
0.906 \\
\end{tabular} & 0.976 & 0.504 & 0.128 \\
\hline & Mean Rank Bogotá & 37.440 & 38.010 & 37.390 & 35.440 & 38.250 & 38.200 & 38.050 & 36.900 & 40.510 \\
\hline & Mean Rank Lisboa & 39.120 & 37.980 & 39.220 & 43.120 & 37.500 & 37.600 & 37.900 & 40.200 & 32.980 \\
\hline \multirow{4}{*}{ LJPR } & Mann-Whitney U & 569.000 & 500.500 & 530.000 & 542.000 & 569.500 & 550.000 & 543.000 & 469.000 & 433.500 \\
\hline & Asymp. Sig. (2-tailed) & 0.474 & 0.137 & 0.258 & 0.324 & 0.517 & 0.375 & 0.319 & 0.068 & 0.025 \\
\hline & Mean Rank Bogotá & 39.120 & 40.490 & 36.100 & 36.340 & 39.110 & 39.500 & 36.360 & 34.880 & 41.830 \\
\hline & Mean Rank Lisboa & 35.760 & 33.020 & 41.800 & 41.320 & 35.780 & 35.000 & 41.280 & 44.240 & 30.340 \\
\hline \multirow{4}{*}{ BOAPA } & Mann-Whitney U & 207.500 & 258 & 309.500 & 278.500 & 271.500 & 293.000 & 237.000 & 212.000 & 218.000 \\
\hline & Asymp. Sig. (2-tailed) & 0.030 & 0.265 & 0.951 & 0.489 & 0.395 & \begin{tabular}{|l|}
0.690 \\
\end{tabular} & 0.127 & 0.040 & 0.058 \\
\hline & Mean Rank Bogotá & 29.700 & 27.680 & 25.620 & 26.860 & 27.140 & 26.280 & 22.480 & 21.480 & 29.280 \\
\hline & Mean Rank Lisboa & 21.300 & 23.320 & 25.380 & 24.140 & 23.860 & 24.720 & 28.520 & 29.520 & 21.720 \\
\hline \multirow{4}{*}{ BOC197 } & Mann-Whitney U & 278.500 & 280.500 & 302.500 & 306.500 & 193.500 & 229.500 & 222.500 & 204.500 & 256.500 \\
\hline & Asymp. Sig. (2-tailed) & 0.466 & 0.463 & \begin{tabular}{|l|}
0.830 \\
\end{tabular} & 0.904 & 0.017 & \begin{tabular}{|l|}
0.025 \\
\end{tabular} & 0.055 & 0.022 & 0.261 \\
\hline & Mean Rank Bogotá & 26.860 & 24.220 & 25.100 & 25.740 & 20.740 & 22.180 & 21.900 & 21.180 & 23.260 \\
\hline & Mean Rank Lisboa & 24.140 & 26.780 & 25.900 & 25.260 & 30.260 & 28.820 & 29.100 & 29.820 & 27.740 \\
\hline \multirow{4}{*}{ BOFA } & Mann-Whitney U & 264.000 & 288.500 & 244.500 & 243.000 & 243.500 & 285.000 & 247.000 & 264.500 & 303.500 \\
\hline & Asymp. Sig. (2-tailed) & 0.317 & 0.613 & 0.168 & 0.156 & 0.160 & 0.580 & 0.165 & 0.332 & 0.856 \\
\hline & Mean Rank Bogotá & 27.440 & 24.540 & 28.220 & 22.720 & 28.260 & 26.600 & 22.880 & 23.580 & 25.860 \\
\hline & Mean Rank Lisboa & 23.560 & 26.460 & 22.780 & 28.280 & 22.740 & 24.400 & 28.120 & 27.420 & 25.140 \\
\hline
\end{tabular}


Tercer Capítulo. Paisajes sonoros: Respuestas y Salidas y su relación con el contexto

\begin{tabular}{|c|c|c|c|c|c|c|c|c|c|c|}
\hline \multirow{4}{*}{ BOPL } & Mann-Whitney U & 286.500 & 269.500 & 268.500 & 229.500 & 242.500 & 236.500 & 276.500 & 230.500 & 242.500 \\
\hline & Asymp. Sig. (2-tailed) & 0.595 & 0.375 & 0.371 & 0.095 & 0.151 & 0.100 & 0.467 & 0.075 & 0.154 \\
\hline & Mean Rank Bogotá & 24.460 & 23.780 & 27.260 & 22.180 & 22.700 & 22.460 & 26.940 & 22.220 & 28.300 \\
\hline & Mean Rank Lisboa & 26.540 & 27.220 & 23.740 & 28.820 & 28.300 & 28.540 & 24.060 & 28.780 & 22.700 \\
\hline \multirow{4}{*}{ BOPSB } & Mann-Whitney U & 208.500 & 213.500 & 203.500 & 305.500 & 263.500 & 218.500 & 196.000 & 183.000 & 233.000 \\
\hline & Asymp. Sig. (2-tailed) & 0.034 & 0.0450 & 0.025 & 0.887 & 0.314 & 0.057 & 0.019 & 0.008 & 0.108 \\
\hline & Mean Rank Bogotá & 29.660 & 29.460 & 21.140 & 25.220 & 27.460 & 29.260 & 20.840 & 20.320 & 28.680 \\
\hline & Mean Rank Lisboa & 21.340 & 21.540 & 29.860 & 25.780 & 23.540 & 21.740 & 30.160 & 30.680 & 22.320 \\
\hline
\end{tabular}


Pasando a los lugares colombianos, BOAPA presenta diferencias estadísticamente significativas en la evaluación general y en el atributo Agitado (Mejor evaluación general en Bogotá y más Agitado en Lisboa). El lugar BOC197 presentó diferencias estadísticamente significativas en los atributos Uniforme/sin eventos, Calmo, Molesto y Agitado (mayores valores de rangos promedios en las evaluaciones de Lisboa para los cuatro atributos), mientras que los lugares BOFA y BOPL no presentaron diferencias estadísticamente considerables en ninguno de sus atributos. Por último, el lugar BOPSB fue donde se obtuvo el mayor número de atributos con diferencias estadísticamente significativas en la evaluación general, Agradable, Caótico, Molesto y Agitado (mayores rangos promedios en evaluación general y Agradable en Bogotá, mientras que se presentaron mayores valores de rangos promedio en Caótico, Molesto y Agitado en Lisboa).

La figura 3-11 presenta la representación cartesiana del modelo de Axelsson según las evaluaciones realizadas en Lisboa y Bogotá. De dicha figura se puede apreciar que, si bien existen pequeños desplazamientos en los ejes X y Y para los diferentes lugares en estudio según su lugar de evaluación, en la mayoría de los casos no se presentan variaciones drásticas, representadas en cambios de cuadrantes para los diferentes lugares en estudio según el lugar de evaluación de los oyentes.

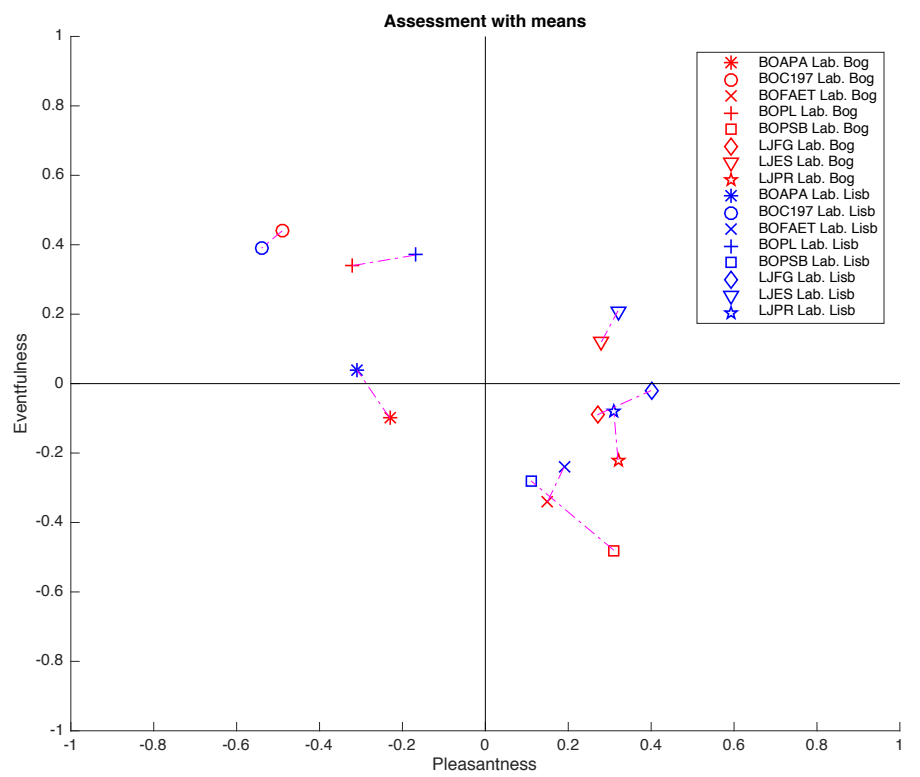

Figura 3-12. Representación gráfica de la evaluación paisajes sonoros de las ciudades de Lisboa y Bogotá según evaluadores bogotanos y lisboetas usando como medida de tendencia central la media. En rojo evaluaciones de bogotanos, en azul evaluación con lisboetas usando como medida de tendencia central las medias. 


\subsubsection{Salidas a largo plazo: diferencias en tiempos de permanencia y uso que} los oyentes de Lisboa y Bogotá.

Las figuras 3-13 y 3-14 presentan los porcentajes de tiempos de permanencia y el uso que los oyentes darían a lugares con entornos acústicos como los que son presentados en las grabaciones, según el lugar de procedencia del evaluador. En algunos casos como los de BOC197 y los tres parques de Lisboa pueden apreciarse ciertas diferencias, aunque para determinar si estas son estadísticamente significativas deben aplicarse las pruebas de Chi-cuadrado y Razón de verosimulitud presentados en la tabla 3-6.

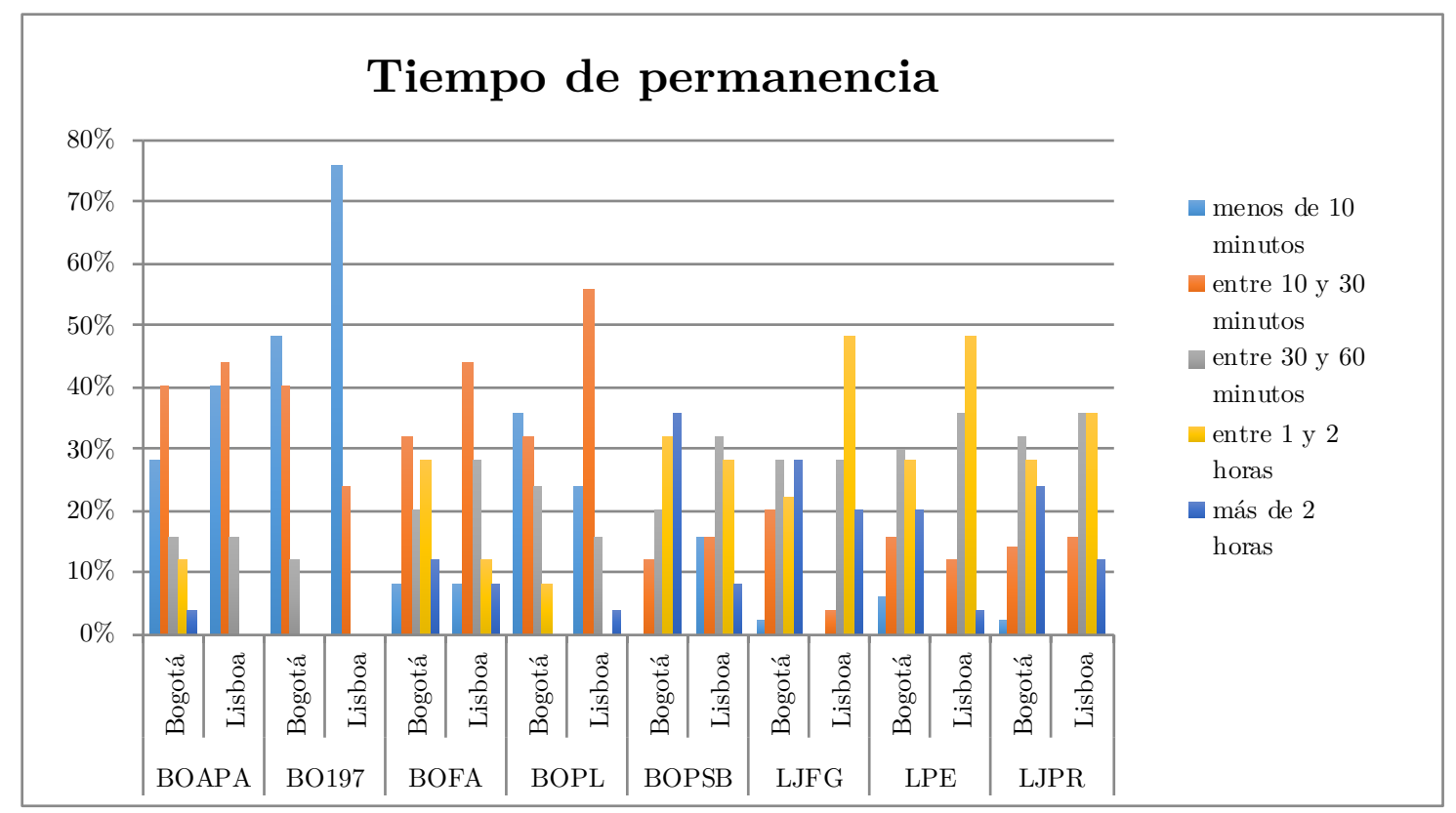

Figura 3-13. Porcentajes de tiempos de permanencia de los evaluadores en lugares con entornos acústicos como los presentados en las grabaciones. 


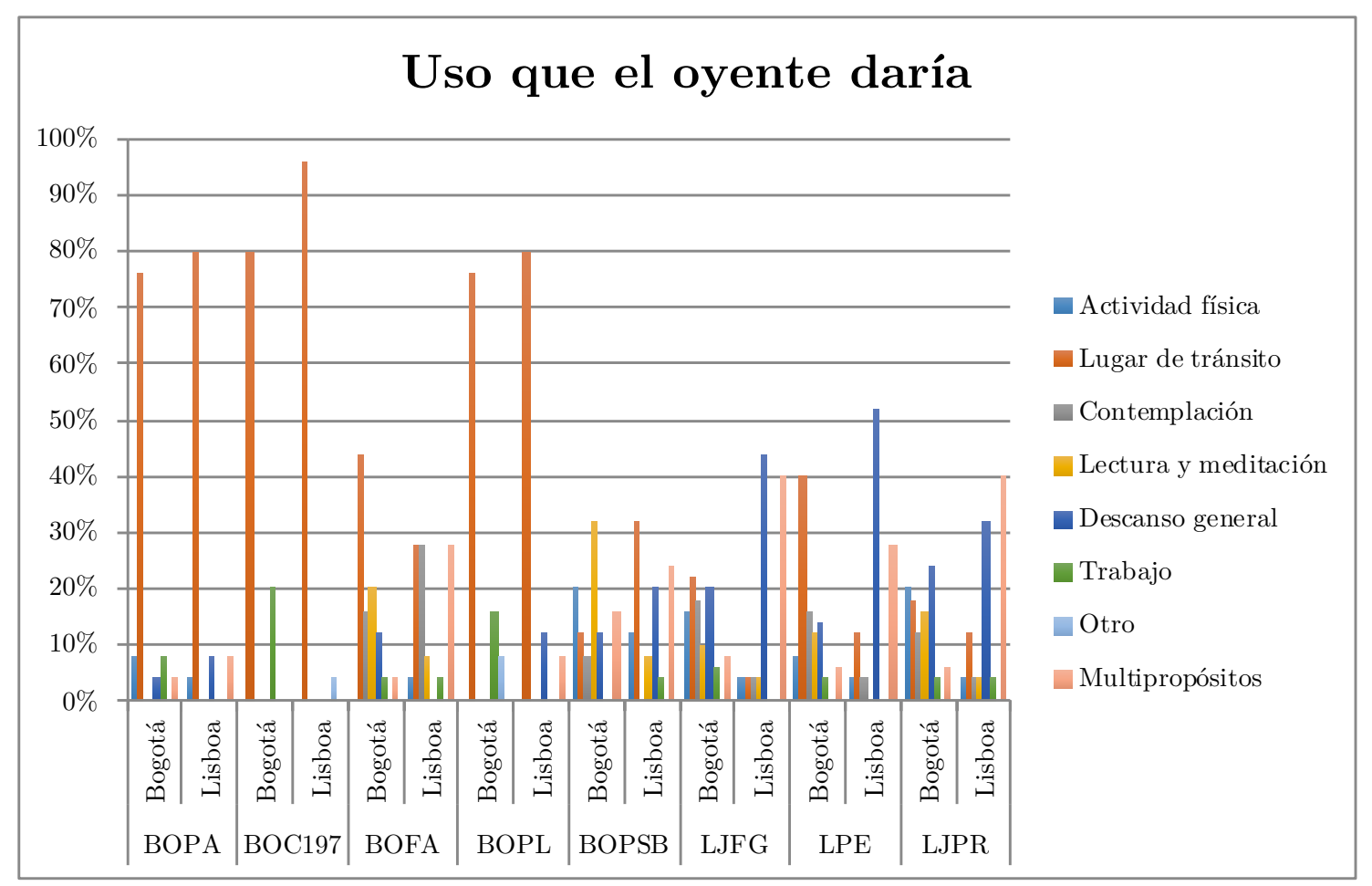

Figura 3-14. Porcentajes de uso que darían los evaluadores en lugares con entornos acústicos como los presentados en las grabaciones.

Tabla 3-22. Resultado de las pruebas de Chi-cuadrado y Razón de verosimilitud para los 8 paisajes sonoros entre evaluadores de Bogotá y evaluadores de Lisboa. Las variables presentadas son tiempos de permanencia y Uso que se daría a lugares con entornos acústicos como los presentados en las grabaciones binaurales.

\begin{tabular}{|c|c|c|c|c|c|c|c|}
\hline \multirow{2}{*}{$\begin{array}{c}\text { Lugar de } \\
\text { prueba }\end{array}$} & \multicolumn{5}{|c|}{ Tiempo de permanencia } & Posible uso \\
\cline { 2 - 9 } & Statistics & Value & $\mathbf{d f}$ & $\begin{array}{c}\boldsymbol{p} \text { value }(2- \\
\text { sided })\end{array}$ & Value & $\mathbf{d f}$ & $\boldsymbol{p}$ value (2-sided) \\
\hline \multirow{3}{*}{ BOAPA } & $\begin{array}{c}\mathrm{X}^{2} \\
\text { Likelihood } \\
\text { Ratio }\end{array}$ & 4.577 & 4 & 0.376 & 3.026 & 4 & 0.770 \\
\hline \multirow{3}{*}{ BOC197 } & $\mathrm{X}^{2}$ & 5.581 & 2 & 0.056 & 6.364 & 2 & 0.050 \\
\cline { 2 - 9 } & $\begin{array}{c}\text { Likelihood } \\
\text { Ratio }\end{array}$ & 6.764 & 2 & 0.044 & 8.682 & 2 & 0.050 \\
\hline \multirow{3}{*}{ BOFA } & $\mathrm{X}^{2}$ & 2.607 & 4 & 0.643 & 11.493 & 6 & 0.050 \\
\cline { 2 - 9 } & $\begin{array}{c}\text { Likelihood } \\
\text { Ratio }\end{array}$ & 2.658 & 4 & 0.658 & 13.66 & 6 & 0.051 \\
\hline & $\mathrm{X}^{2}$ & 5.636 & 4 & 0.198 & 11.026 & 4 & 0.008 \\
\cline { 2 - 8 } & $\begin{array}{c}\text { Likelihood } \\
\text { Ratio }\end{array}$ & 6.823 & 4 & 0.181 & 15.275 & 4 & 0.006 \\
\hline \multirow{3}{*}{ BOPSB } & $\mathrm{X}^{2}$ & 9.356 & 4 & 0.049 & 10.273 & 6 & 0.094 \\
\cline { 2 - 8 } & $\begin{array}{c}\text { Likelihood } \\
\text { Ratio }\end{array}$ & 11.272 & 4 & 0.038 & 11.785 & 6 & 0.093 \\
\hline
\end{tabular}


Tercer Capítulo. Paisajes sonoros: Respuestas y Salidas y su relación con el contexto

\begin{tabular}{|c|c|c|c|c|c|c|c|}
\hline \multirow{4}{*}{ LJFG } & $\mathrm{X}^{2}$ & 7.504 & 4 & 0.096 & 22.646 & 6 & 0.000 \\
\cline { 2 - 8 } & $\begin{array}{c}\text { Likelihood } \\
\text { Ratio }\end{array}$ & 8.300 & 4 & 0.086 & 24.590 & 6 & 0.001 \\
\hline \multirow{4}{*}{ LJES } & $\mathrm{X}^{2}$ & 6.701 & 4 & 0.155 & 25.736 & 6 & 0.000 \\
\cline { 2 - 8 } & $\begin{array}{c}\text { Likelihood } \\
\text { Ratio }\end{array}$ & 8.239 & 4 & 0.110 & 28.267 & 6 & 0.000 \\
\hline \multirow{3}{*}{ LJPR } & $\mathrm{X}^{2}$ & 2.173 & 4 & 0.773 & 17.942 & 6 & 0.004 \\
\cline { 2 - 8 } & $\begin{array}{c}\text { Likelihood } \\
\text { Ratio }\end{array}$ & 2.584 & 4 & 0.740 & 18.474 & 6 & 0.009 \\
\hline
\end{tabular}

A la pregunta relacionada con el tiempo de permanencia, de los 8 lugares en estudio, solo dos presentaron asociación estadísticamente significativa entre el lugar de evaluación y esta variable. Los evaluadores de Bogotá permanecerían más tiempo en los lugares BOC197 y BOPSB que los evaluadores de Lisboa.

Respecto al uso que se daría a un lugar con un entorno sonoro como el presentado en la grabación binaural, 5 de los 8 lugares en estudio presentaron asociación estadísticamente significativa entre esta variable y el lugar de evaluación. Resulta interesante que de los 3 lugares que no presentaron asociación estadísticamente significativa entre las variables Lugar de prueba y Posibles usos, dos son lugares que pueden ser considerados lugares transversales a cualquier ciudad (una fuente de agua BOFA y una autopista BOAPA), mientras que el tercer lugar que no presenta asociación entre estas variables es el parque BOPSB, aunque como se mencionó anteriormente, este lugar si presenta asociación entre las variables tiempo de permanencia y lugar de prueba.

Continuando con el análisis de las variables Posible uso y Lugar de prueba, los resultados presentados permiten apreciar un segundo grupo de lugares (los parques de Lisboa LJFG, LPE, LPR, y los lugares BOC197 y BOPL de Bogotá), los cuales si presentan asociación estadísticamente significativa entre las variables Lugar de prueba y Posibles usos. De forma específica, si bien el lugar BOPL presenta los mayores porcentajes en el uso Lugar de tránsito para los dos lugares de evaluación (76\% en Bogotá y $80 \%$ en Lisboa), en Bogotá también se usaría este lugar para trabajo, mientras que en Lisboa lo usarían para descanso general. Estos mismos resultados se repiten en el lugar BOC197. En el lugar LJFG las diferencias son más marcadas, dado que las evaluaciones de Lisboa muestran que este lugar se usaría principalmente como lugar de descanso general y un lugar 
multipropósito, mientras que en Bogotá los porcentajes principales se reparten entre Lugar de tránsito, descanso general y contemplación. Resultados similares fueron encontrados en el lugar LPE, donde las evaluaciones de Lisboa muestran un uso principalmente relacionado con el descanso general, mientras que de nuevo en Bogotá se usaría principalmente como lugar de tránsito. Por último, el lugar LJPR para los evaluadores de Lisboa sería usado principalmente como lugar multipropósito y lugar de descanso general, mientras que en Bogotá se usaría como lugar de descanso y lugar de tránsito.

\subsection{Discusión y conclusiones.}

A fin de aportar claridad en la discusión y conclusiones, estas se han dividido según el tipo de prueba realizada.

3.4.1 Discusión y conclusiones según comparación entre evaluaciones in situ vs. laboratorio.

Fueron encontradas diferencias significativas en las evaluaciones de paisajes sonoros según los métodos de evaluación subjetiva in situ y en laboratorio. En los casos de estudio estas diferencias se vieron manifestadas principalmente en los atributos Caótico, Agitado y Uniforme/sin eventos, generando un impacto considerable en los componentes principales Pleasantness y Eventfulness del modelo de atributos perceptuales propuesto por Axelsson. Se considera que estos cambios pueden presentarse debido a dos razones principales: la existencia de diversos estímulos alternativos en las evaluaciones in situ (visuales, olfativos, táctiles y gustativos) y en la variabilidad de la composición de los paisajes sonoros en las pruebas in situ respecto a las grabaciones presentadas en las pruebas en laboratorio.

Respecto a la diversidad de estímulos existentes en las pruebas in situ, se considera que aspectos visuales son altamente influyentes en los resultados obtenidos. En los casos de estudio, aplicado a parques urbanos de la ciudad de Lisboa, el entorno visual genera una demarcación espacial entre "la ciudad" y "el parque". Esta envolvente visual se manifestó en los resultados de las evaluaciones, donde los entornos sonoros eran más agradables y calmos in situ que en laboratorio. Estos acontecimientos hacen pensar que los estímulos visuales 
permiten crear una especie de "barrera acústica", la cual, a pesar de ser de tipo visual, afecta la evaluación del entorno acústico. Sin embargo, en los laboratorios dicha barrera no existe y para la evaluación solo se tiene como variable el estímulo sonoro (marcadamente variable y renovable en aspectos temporales), por lo que los evaluadores pueden apreciar entornos más caóticos y agitados producto de las actividades típicas de la ciudad.

Pasando a la variabilidad de los paisajes sonoros en las pruebas in situ, comparadas con el número controlado de eventos sonoros de las pruebas en laboratorio, es interesante ver cómo las desviaciones estándar de las pruebas in situ son mayores que las de laboratorio, generando también distribuciones menos simétricas en las pruebas in situ respecto a las pruebas de laboratorio. En las pruebas in situ, cada oyente tardaba entre 10 y 20 minutos en responder el cuestionario, tiempo suficiente para percibir una cantidad de eventos sonoros mucho mayor a la presentada en fragmentos de audio de treinta segundos. Así mismo, el proceso total de evaluación de cada parque podía tardar al menos cuatro horas (tiempo requerido para realizar la evaluación a cada una de las 20 personas), lo que incrementaba la ventana temporal de evaluación total para cada parque, por lo que era imposible que cada uno de los participantes evaluara exactamente el mismo entorno acústico. Esto contrasta con la prueba en laboratorio, donde todos los evaluadores evaluaron exactamente el mismo fragmento de audio.

Se considera que si bien las pruebas in situ permiten una evaluación sistémica y holística de los entornos acústicos, las pruebas en laboratorio permiten realizar un análisis puntual y específico de diferentes eventos sonoros que componen los entornos acústicos, por lo que las dos metodologías pueden ser usadas en trabajos de evaluación de paisajes sonoros dependiendo de la aplicación y necesidades que se tengan, siendo potencialmente útiles y complementarias en el diseño, evaluación y gestión de entornos acústicos urbanos.

Salvo el lugar LJFG, no fueron encontradas diferencias apreciables en la evaluación general de los entornos acústicos según el tipo de metodología de evaluación (in situ vs laboratorio), lo que contrasta con el comportamiento ya descrito de los atributos perceptuales más específicos usados por el modelo de Axelsson. La primera que debido a que todos los oyentes conocían los lugares en evaluación, las experiencias previas pueden afectar la valoración global de entorno 
acústico, independiente del tipo de prueba realizada. La segunda que cuando se empieza a indagar respecto a aspectos más específicos de los entornos acústicos, las diferencias entre metodologías de evaluación empiezan a ser considerables.

Así mismo no fueron encontradas diferencias estadísticamente significativas entre los evaluadores expertos y no expertos para las pruebas de laboratorio. Es posible que este resultado esté relacionado con que todos los evaluadores conocían los lugares en estudio, por lo que tenían una familiaridad con el entorno. Esto implica que, en las pruebas de evaluación de paisajes sonoros, existe una relación entre los evaluadores y los entornos acústicos en análisis, facilitando por ende la evaluación subjetiva independiente de la experticia de los jueces. La visita y conocimiento de los lugares a evaluar, así como el contacto simple con el entorno acústico urbano puede ser considerado como un proceso natural de entrenamiento mediado por el contexto, marcando una diferencia entre la evaluación de paisajes sonoros y la evaluación de calidad acústica de sonidos específicos (donde se recomienda el entrenamiento previo de los evaluadores).

Se encontró que los resultados de oyentes expertos y no expertos estaban en línea con los hallazgos de trabajos previos [31], en el sentido de que muestran la importancia de aspectos contextuales en la evaluación de los paisajes sonoros urbanos. De hecho, en el trabajo de Liu [31], los evaluadores expertos y turísticos tuvieron experiencias y expectativas previas totalmente diferentes, mientras que en este trabajo todos los oyentes tuvieron experiencias previas con los parques en estudio. Los antecedentes y el contexto de los oyentes son, por lo tanto, un factor importante a tener en cuenta en la evaluación del paisaje sonoro.

3.4.2 Discusión y conclusiones de diferencias en respuestas y salidas según evaluadores de Lisboa y Bogotá.

Los resultados encontrados respecto a aspectos descriptivos muestran que los oyentes de los dos países identificaron los mismos sonidos fundamentales para siete de los ocho lugares estudiados. Esto puede significar que, para los casos en estudio, la posibilidad de identificar el sonido del sonido fundamental no se ve afectada por la interacción lugar-persona. Sin embargo, es interesante que, aunque no se encontraron diferencias en este aspecto descriptivo, se encontraron algunas diferencias en las respuestas y salidas según el lugar de origen del oyente. Esto 
puede indicar que una cosa es la capacidad de detectar e identificar el sonido fundamental de los entornos acústicos y otra es el significado que les dan los oyentes a dichos sonidos.

Del mismo modo, si bien está claro que los niveles altos de LAeq afectan la salud de las personas (lo que implica que un primer paso para la gestión acústica urbana es el control y la reducción de estos niveles sonoros), no necesariamente los niveles de energía bajos pueden garantizar un tiempo de permanencia más prolongado en algunos lugares, o un uso específico (salidas). Por ejemplo, BOFAET presentó los valores más altos de LAeq, pero era un lugar donde la mayoría de las personas estarían entre 10 y 30 minutos. Sin embargo, los lugares con mayor LAeq si fueron considerados más molestos por los oyentes lo que concuerda con resultados de trabajos previos, donde los parámetros asociados con los niveles de energía influyen en el grado de confort y/o molestia [50-54].

Además, para las respuestas a corto plazo (atributos perceptuales) de los ocho lugares en estudio, tres presentaron diferencias estadísticamente significativas en el parámetro Agitado, mientras que los atributos Monótono, Calmo y Desagradable presentaron diferencias significativas en solo dos lugares. La evaluación general también presentó diferencias en dos lugares, mientras que atributos como Agradable, Caótico, Excitante y Uniforme/sin eventos solo presentaron diferencias estadísticamente significativas en uno de los lugares en estudio. Como ha sido expuesto en el capítulo 2, estos ocho componentes impactan de forma directa la estimación de los componentes principales Eventfulness y Pleasantness del modelo de Axelsson, por lo que si son pocas las asociaciones entre el lugar de prueba del oyente y los atributos perceptuales, existirá poca afectación en la representación cartesiana por cuadrantes aplicada en este modelo (representada en cambios significativos de cuadrantes en el modelo).

Dado que la variable Lugar de Prueba implica un conocimiento previo de los lugares por parte de las personas involucradas en los experimentos, existe, en consecuencia, una interacción entre la Persona y los lugares en estudio (actividades y características). Por lo tanto puede decir que, en las condiciones de estas pruebas, existe una relación entre las respuestas (aspectos actitudinales) y la interacción lugar-persona. Por lo tanto, a partir de los conocimientos y experiencias previos en lugares, es posible generar diferencias en las respuestas de 
los oyentes (por ejemplo, para el lugar de LJFG, los oyentes de Lisboa lo consideraron más Excitante, mientras que los evaluadores de Colombia lo calificaron como Monótono).

Al pasar a las salidas de largo plazo (tiempo de permanencia y uso), se encontró que 5 de los 8 lugares en estudio presentaban dependencia entre las variables lugares de prueba de los oyentes y los posibles usos, aunque solo dos presentaron dependencia entre las variables lugar de prueba del oyente y tiempo de permanencia. Estos resultados muestran que, bajo las condiciones planteadas para el desarrollo de este experimento, las salidas a largo plazo presentan una dependencia según la interacción lugar-persona. Para comprender mejor estos resultados, el enfoque de la semiótica puede ser útil, pues los sitios, el uso, el sonido y el significado tienen una relación directa.

Los significados de los lugares dependen del uso que se da a estos $($ significado $=$ uso) [55], razón por la cual son los usuarios quienes son capaces de realizar la lectura de un lugar y de darle su significado. Por lo tanto, un lugar puede considerarse un "centro de significado" que se nutre de las experiencias y los apegos de las personas [56]. Bajo este análisis, no es posible que lugares representativos de cada ciudad (caso de los parques de Lisboa o Bogotá) puedan tener el mismo significado para evaluadores de países diferentes, cuando los evaluadores no conocen ni han usado estos lugares. Por lo anterior, si bien es cierto que las grabaciones de los entornos sonoros dan una idea de las actividades y prácticas desarrolladas en el lugar, es imposible que estas aporten información relacionada con el uso del lugar según el contexto, más allá de transmitir información de prácticas o actividades desarrolladas. La interacción lugar-persona es producto de la experimentación directa de la persona, lo que genera memorias y experiencias previas que alimentan las expectativas y las actividades a desarrollarse en el lugar. Bajo condiciones de laboratorio y sin estas experiencias previas, los oyentes asociarán los entornos acústicos a sus propias experiencias con lugares de características diferentes, lo que generará una diferencia de significados según el lugar de procedencia del oyente.

Así mismo, es interesante ver la aparición de lugares que pueden ser considerados comunes o transversales a cualquier ciudad (una autopista o una fuente de agua), esto dado que no se presenta dependencia entre las variables 
posible uso y lugares de prueba del oyente, lo que quiere decir que su "significado" es el mismo independiente del lugar de evaluación del oyente. Es aquí cuando el concepto de los no lugares puede ser útil en la discusión de estos resultados. Un lugar puede definirse como "lugar de identidad, relacional e histórico", por lo que un espacio que "no puede definirse ni como lugar de identidad ni como relacional ni como histórico es considerado como un no lugar" [57]. Acercando este concepto al campo de los paisajes sonoros, entornos sonoros que no representen o generen identidad, evoquen algún recuerdo o despierten algún sentido de pertenencia en el oyente pueden ser considerados como no lugares sonoros. Para profundizar en este concepto, se presentará el detalle de dos de los ocho casos presentados en este estudio: los lugares BOAPA (autopista norte de Bogotá) y LJPE (parque urbano de la ciudad de Lisboa).

El lugar BOAPA es la principal autopista de Bogotá y comunica la capital colombiana con el norte del país. Esta autopista cruza parte de la ciudad en sentido sur-norte y norte-sur y por ella pueden llegar a transitar en horas pico unos 14000 vehículos por hora (sumando los dos sentidos). Como principal particularidad debe resaltarse que en parte de su trazado cuenta con carriles exclusivos por donde circulan buses biarticulados del sistema de transporte masivo. Por lo demás, es una autopista común que, si bien está ubicada en Bogotá, también podría estarlo en cualquier parte del mundo. Su uso es el de cualquier otra autopista: una vía de comunicación por donde circulan vehículos. Podría ser el ejemplo perfecto de un no lugar sonoro, dado que las fuentes sonoras que lo componen y los usos que se le da a este espacio no varía dependiendo de la ciudad. Al aplicar las pruebas de dependencia entre las variables Posible uso y Lugar de prueba, se encuentra que efectivamente el uso que se le daría a un lugar con un entorno sonoro como el presentado en la grabación sería el de lugar de paso tanto para los evaluadores de Lisboa como para los de Bogotá. Esto implica que para los dos grupos de evaluadores el lugar tiene el mismo uso y por ende el mismo significado.

Pasando al lugar LJE, este parque ubicado en el barrio Lapa de la ciudad de Lisboa fue construido a finales del siglo XIX. En sus 4,6 ha se cuenta con dos lagos, parques infantiles, kioscos y equipamiento para ejercitase físicamente. En los lagos pueden verse patos, cisnes y gansos, y rondando el jardín se encuentra 
un majestuoso pavo real. En el centro del jardín se cuenta con una glorieta en hierro que es usada generalmente para conciertos de bandas filarmónicas y disfrute general. Una de sus calles colinda con la Basílica da Estrela de Lisboa, punto obligado para turistas que además pueden llegar al lugar en el mítico tranvía de madera E28. En épocas de verano son comunes conciertos y picnics de lisboetas y turistas que llegan a disfrutar de este espacio. Las grabaciones binaurales presentadas a los evaluadores registraban el sonar de las campanas de la Basílica, los sonidos de los pájaros y de la naturaleza, así como sonido del transporte público. Al analizar los resultados obtenidos para las variables Posible uso y Lugar de prueba, se encuentra que estas si tienen una asociación estadísticamente significativa: mientras Bogotanos lo usarían principalmente como lugar de tránsito, Lisboetas lo usarían como lugar de descanso general. Es claro en este caso que los usos, la historia y el contacto directo con el lugar impactan en su significado y, dada la descontextualización de los evaluadores de Bogotá, y los múltiples significados que puede tener ese espacio para los lisboetas, los significados según los lugares de evaluación son diferentes.

De esta forma, bajo las condiciones de laboratorio usadas en este estudio, se considera que las respuestas no generaron asociaciones considerables con el lugar de prueba. Sin embargo, salidas a largo plazo si presentaron asociaciones con el lugar de prueba. Esto último está relacionado con dos grandes conceptos: 1) la asociación del significado a partir del uso y 2) la aparición de los no lugares sonoros.

La asociación del significado del lugar al posible uso, así como la relación de estos aspectos con los paisajes sonoros, demuestran la necesidad de realizar estudios con enfoques multidisciplinarios en este campo. En esta tesis doctoral, además de considerar aspectos de la percepción ecológica, se analizaron los ambientes acústicos como portadores de significado, que adquieren significado a partir de aspectos contextuales. Bajo las condiciones de este estudio, se estableció que para la formación del significado de un ambiente acústico las experiencias previas de las personas, las actividades para las cuales se creó ese espacio y los aspectos culturales y espaciales que influyen en las prácticas desarrolladas en los lugares, deben ser consideradas. Es decir, la interacción lugar-persona afecta el significado de los entornos acústicos a partir de su uso. 
Así mismo, la homogenización de lugares también impacta los paisajes sonoros, y si se tienen fuentes sonoras similares y usos de lugares similares, los paisajes sonoros también serán similares. Esto da lugar a los no lugares sonoros. Lugares que no presentan diferencia en su significado independiente del lugar de procedencia del oyente como centros comerciales, aeropuertos, grandes vías. Por supuesto, no es posible pensar que todos los lugares deban tener una identidad única, aunque si es importante preservar y conservar aquellos espacios que así lo requieran.

3.4.3 Aspectos contextuales y semióticos aplicados a procesos de gestión, diseño y planeación de paisajes sonoros urbanos.

Las ciudades son un reflejo de sus habitantes y su cultura, por lo que en ella se pueden encontrar diferentes formas de expresión representadas por diferentes lenguajes. Por lo tanto, cada acción se convierte potencialmente en un signo que será descifrado por las personas de acuerdo con su repertorio y contexto. En este sentido, la ciudad se comunica, transmite información [58], y el lenguaje sonoro es un poderoso portador de significado. Dado que los entornos acústicos son el producto de prácticas y actividades desarrolladas por personas, el concepto de paisaje sonoro constituye otro enfoque para relacionar y comprender la ciudad. El objetivo, por lo tanto, en los procesos de diseño, planificación y gestión de espacios urbanos, consiste en diseñar e implementar entornos acústicos urbanos que transmitan información coherente a las personas para que asignen un significado a los signos según el espacio.

Así mismo, si un lugar adquiere "significado" según su uso, el "significado" del entorno acústico se verá afectado por la interacción lugar-persona (aspectos contextuales). Cuando se realiza un ejercicio inverso en los procesos de diseño, planificación y gestión, vale la pena preguntar: ¿cuál es el "Uso-Significado" del lugar? Este ejercicio implica la comprensión del proceso semiótico donde tres aspectos, Lugar, Entorno acústico y Paisaje sonoro, están relacionados con el contexto para generar un "significado", es decir, implica un modelo de semiosis aplicado al paisaje sonoro urbano.

El concepto de semiosis se puede comprender como un proceso de selección, organización, coordinación y estructuración de los ítems de percepción y objetos 
de experiencia [39], la Figura 3-15 presenta el núcleo de un modelo de semiosis ajustado para paisajes sonoros. El propósito del modelo se basa en hallazgos previos en los campos de la calidad del sonido, la semiótica y el diseño del paisaje sonoro [39-41,59,60], donde el Lugar, el Entorno acústico y el Paisaje sonoro están relacionados, teniendo a la Persona como eje central. El modelo de semiosis propuesto en esta tesis se basa en el modelo triádico general propuesto por Peirce, que fue adaptado por Jekosch para los procesos de calidad de sonido. En esta adaptación, el entorno acústico es un portador de signos que representa un lugar específico (objeto), el paisaje sonoro es el resultado del proceso cognitivo desarrollado por una persona, que adquiere significado del contexto (que a su vez influye en la ciudad, las personas) y los ambientes acústicos).

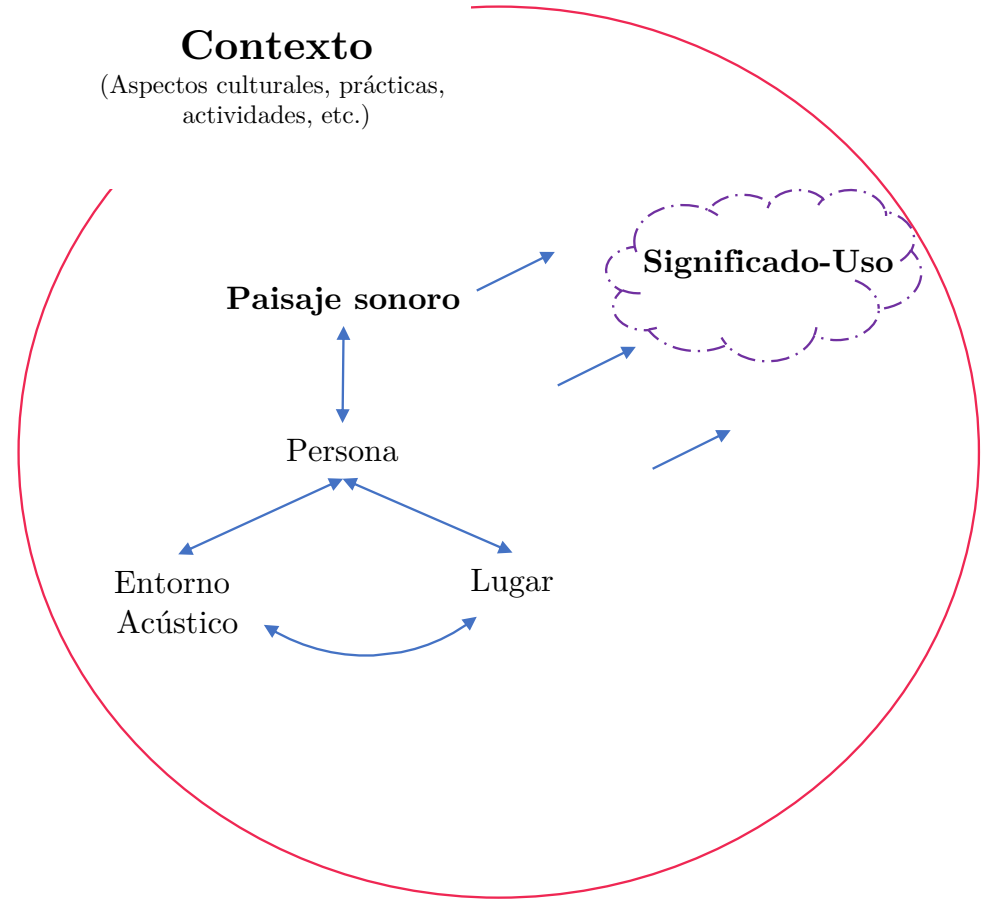

Figura 3-15. Modelo de semiosis de paisajes sonoros urbanos [61]

El proceso presentado en la Figura 3-15, establece una conexión entre el Lugar, el entorno Acústico y el Paisaje Sonoro resultante, y va en la misma línea de trabajos anteriores, que indican la necesidad de relacionar el uso de los espacios con los paisajes sonoros $[29,60]$. Las personas interactúan con los lugares, por lo que el entorno acústico se configura desde el affordance del lugar, las actividades y las prácticas desarrolladas en los mismos. El paisaje sonoro es, por lo tanto, un 
producto de la interacción de la persona con su lugar y su entorno acústico, que a su vez se alimenta del contexto y contribuye al significado de los lugares.

Considerando el hecho de que el enfoque de la gestión de paisajes sonoros amplía la gestión acústica tradicional, debido a que su objetivo principal es el bienestar de las personas, esta sección de la tesis profundiza en la necesidad de administrar paisajes sonoros para mejorar la experiencia ambiental de las personas (de acuerdo con los criterios del lugar y las respuestas y salidas deseadas) y contribuir al fortalecimiento del significado de los lugares.

Por lo anterior, el proceso de diseño del paisaje sonoro se puede profundizar al plantear preguntas relacionadas con diferentes aspectos contextuales y semióticos. Sus respuestas pueden proporcionar información para los criterios de evaluación, diseño, gestión y planificación de entornos acústicos, de acuerdo con la hoja de ruta de diseño de paisajes sonoros de Bento-Coelho [77].

Tabla 3-23. Aspectos contextuales y semióticos aplicado a la guía de diseño de paisajes sonoros de Bento-Coelho

\begin{tabular}{|l|}
\hline \begin{tabular}{c} 
Definir propuestas y actividades \\
$\bullet \quad$ ¿Cual es el objetivo y/o el significado deseado del lugar? \\
\hline Definir objetivos acústicos acorde a la propuesta y actividades establecidos \\
$\bullet \quad$ ¿Cómo contribuye el entorno acústico a la experiencia ambiental y/o al \\
significado del lugar? ¿Cual es la relación persona-lugar? (incluyendo \\
expectativas, uso y apropiación de personas)? \\
\hline Identificar lugares de escucha e itinerarios de escucha \\
¿Qué actividades se realizan en los lugares? (según la época del año, hora, etc.) \\
\hline Identificar fuentes de sonido y componentes sonoros \\
¿Qué sonidos identifican o representan las actividades más características del \\
lugar? \\
¿Qué sonidos mejoran la experiencia ambiental y son coherentes con el contexto, \\
el significado y el uso del lugar? \\
\hline Identificar rutas de propagación de sonido. \\
¿Cómo interactúan los sonidos y las fuentes de sonido con su entorno? \\
\hline Identificar sonidos preferidos y no deseados \\
¿Qué sonidos no favorecen la experiencia ambiental, son incoherentes con el \\
contexto, el significado y el uso del lugar? \\
\hline Administre componentes de sonido (disminuya los sonidos no deseados, mejore los sonidos \\
preferidos e identifique los sonidos deseados en contexto) \\
¿Cómo mejorar y / o crear sonidos que favorezcan la experiencia ambiental, el \\
uso y el significado del lugar? \\
¿Cómo reducir los sonidos que no favorecen la experiencia ambiental, en \\
detrimento del uso y significado del lugar?
\end{tabular} \\
\hline
\end{tabular}


A la luz de los resultados presentados en este capítulo puede sugerirse que, en los procesos de diseño, planificación y gestión de la acústica ambiental, no solo son necesarios los análisis taxonómicos, energéticos, temporales y espaciales de los entornos acústicos, sino también de estudios contextuales y semiótico del espacio urbano. En este sentido, el análisis del uso del lugar, de las personas que usarán el espacio, de la coherencia entre el espacio y el entorno acústico y el significado deseado, pueden ser en el desarrollo de este tipo de proyecto.

Los resultados de este trabajo sugieren la necesidad de continuar ampliando los procesos de gestión, diseño y planificación de paisajes sonoros urbanos, a partir de considerar aspectos contextuales y semióticos. Estos componentes permiten una mejor comprensión de la experiencia ambiental y fortalecen el significado de los lugares, a fin de asegurar aún más el bienestar de las personas. De esta manera, las preguntas abiertas en este trabajo pueden complementar los enfoques tradicionales y enriquecer los procesos de gestión y diseño acústico. Estas preguntas, junto con el modelo de semiosis propuesto, pueden contribuir concretamente a crear los insumos necesarios para tales procesos.

Finalmente, los diseñadores acústicos urbanos tienen la responsabilidad de presentar información coherente entre el entorno acústico y el uso del lugar. Esto, a su vez, implica definir estrategias multidisciplinares que establezcan cómo y cuándo se genera la información, para ayudar en el proceso de formación del significado de los lugares por parte de los oyentes. 


\section{Referencias Tercer Capítulo}

1. Davies, W.J.; Adams, M.D.; Bruce, N.S.; Marselle, M.; Cain, R.; Jennings, P.A.; Poxon, J.; Carlyle, A.; Cusack, P.; Hall, D.A.; et al. The Positive Soundscape Project: A synthesis of results from many disciplines. In 38th International Congress and Exposition on Noise Control Engineering 2009, INTER-NOISE 2009; Ottawa, 2009; Vol. 1, pp. 663-672.

2. Davies, W.J.; Adams, M.D.; Bruce, N.S.; Cain, R.; Carlyle, A.; Cusack, P.; Hall, D.A.; Hume, K.I.; Irwin, A.; Jennings, P.; et al. Perception of soundscapes: An interdisciplinary approach. Appl. Acoust. 2013, 74, 224231, doi:10.1016/j.apacoust.2012.05.010.

3. Brown, A.L.; Kang, J.; Gjestland, T. Towards standardization in soundscape preference assessment. Appl. Acoust. 2011, 72, 387-392, doi:10.1016/j.apacoust.2011.01.001.

4. International Standar Organization (ISO) 12913-1 Acoustics - Soundscape - Part 1: Definition and conceptual framework 2013.

5. Schulte-Fortkamp, B.; Fiebig, A. Impact of Soundscape in Terms of Perception. In Soundscape and the Built Environment; Kang, J., SchulteFortkamp, B., Eds.; CRC Press, 2016; pp. 69-88.

6. Jeon, J.Y.; Hong, J.Y.; Lavandier, C.; Lafon, J.; Axelsson, Ö.; Hurtig, M. A cross-national comparison in assessment of urban park soundscapes in France, Korea, and Sweden through laboratory experiments. Appl. Acoust. 2018, 133, doi:10.1016/j.apacoust.2017.12.016.

7. Brown, A.L.; Gjestlan, T.; Dubois, D. Acoustic Environments and soundscapes. In Soundscape and the Built Environment; Kang, J., SchulteFortkamp, B., Eds.; 2016; pp. 1-16.

8. International Standar Organization (ISO) ISO/TS 12913-2:2018. Acoustics - Soundscape - Part 2: Data collection and reporting requirements 2018, 140.

9. Gibson, J.J. The ecological approach to visual perception; Taylor \& Francis: New York, 1986;

10. Lillo, J. Ecología Perceptiva: Aportaciones Y Limitaciones. Anu. Psicol. 1987, 36/37, 21-40.

11. Gibson, J.J. Percepción del mundo visual; Revol, E., Ed.; Ediciones Infinito: Buenos Aires, 1974;

12. Gibson, J.J. The Ecological Approach To the Visual Perception of Pictures. Leonardo 1978, 11, 227-235.

13. Lutfi, R.A. Human Sound Source Identification. In Auditory Perception of Sound Sources; Yost, W., Popper, A., Fay, R., Eds.; Springer: New York, 
2008; Vol. 16, p. 545 ISBN 9780387714677.

14. Meilgaard, M.C.; Carr, B.T.; Civille, G.V. Sensory Evaluation Techniques, Fourth Edition; Third.; CRC Press: New York, 2000; ISBN 0-84930-276-5.

15. Schiffman, H. Sensation and Perception: An Integrated Approach; 4th editio.; John Wiley \& Sons: New York, 2001; ISBN 0471249300.

16. Herranz-Pascual, K.; Aspuru, I.; García, I. Proposed conceptual model of environmental experience as framework to study the soundscape. In Proceedings of Internoise 2010. Noise and sustainability; Lisbon, 2010.

17. Pol, E.; Valera, S.; Vidal, T. Psicología ambiental y procesos psicosociales. In Psicología Social; Morales, F., Huici, C., Eds.; Madrid, 1999; pp. 235252 ISBN 8448124359.

18. Briñol, P.; Falces, C.; Becerra, A. Actitudes. In Psicología Social; Morales, J.F., Moya, M.C., Gaviria, E., Cuadrado, I., Eds.; McGraw-Hill: Madrid, 2007; pp. 258-260 ISBN 978-84-481-5608-4.

19. Aspuru, I.; Garcia, I.; Herranz-Pascual, K.; Garcia-Borreguero, I. Understanding Soundscape as a specific Environmental Experience: Highlighting the importance of context relevance. 2012, 14, 015004-015004, doi:10.1121/1.3676685.

20. Javaloy, F.; Vidal, T. Bases ambientales del comportamiento. In Psicología Social; Morales, J.F., Moya, M., Gaviria, E., Cuadrado, I., Eds.; McGrawHill: Madrid, 2007 ISBN 978-84-481-5608-4.

21. Herranz-Pascual, K.; García, I.; Aspuru, I.; Díez, I.; Santander, Á. Progress in the understanding of soundscape: objective variables and objectifiable criteria that predict acoustic comfort in urban places. Noise Mapp. 2016, 3, 247-263, doi:10.1515/noise-2016-0017.

22. Garcia, I.; Herranz-Pascual, K.; Aspuru, I.; Gutierrez, L.; Acero, J.A.; Santander, A. Indicators of Environmental Comfort Sensitive to Human Perception. In Handbook of Research on Perception-Driven Approaches to Urban Assessment and Design; Aletta, F., Xiao, J., Eds.; 2018; pp. 508-533 ISBN 9781522536376.

23. Jeon, J.Y.; Lee, P.J.; Hong, J.Y.; Cabrera, D. Non-auditory factors affecting urban soundscape evaluation. J. Acoust. Soc. Am. 2011, 130, 3761-70, doi:10.1121/1.3652902.

24. Pheasant, R.; Horoshenkov, K.; Watts, G.; Barrett, B. The acoustic and visual factors influencing the construction of tranquil space in urban and rural environments tranquil spaces-quiet places? J. Acoust. Soc. Am. 2008, 123, 1446-1457, doi:10.1121/1.2831735.

25. Liu, J.; Kang, J.; Behm, H.; Luo, T. Effects of landscape on soundscape perception: Soundwalks in city parks. Landsc. Urban Plan. 2014, 123, 30- 
40, doi:10.1016/j.landurbplan.2013.12.003.

26. Truax, B.; Barrett, G.W. Soundscape in a context of acoustic and landscape ecology. Landsc. Ecol. 2011, 26, 1201-1207, doi:10.1007/s10980-011-96449.

27. Liu, J.; Kang, J.; Luo, T.; Behm, H. Landscape effects on soundscape experience in city parks. Sci. Total Environ. 2013, 454-455, 474-481, doi:10.1016/j.scitotenv.2013.03.038.

28. Herranz-Pascual, K.; García, I.; Diez, I.; Santander, A.; Aspuru, I. Analysis of Field Data to Describe the Effect of Context ( Acoustic and Non-Acoustic Factors ) on Urban Soundscape. Appl. Sci. 2017, \%, 1-18, doi:10.3390/app7020173.

29. Hong, J.Y.; Jeon, J.Y. Landscape and Urban Planning Influence of urban contexts on soundscape perceptions: A structural equation modeling approach. Landsc. Urban Plan. 2015, 141, 78-87, doi:10.1016/j.landurbplan.2015.05.004.

30. Romero, V.P.; Brambilla, G.; Gabriele, M. Di; Gallo, V.; Maffei, L. The influence of the soundscape on the tourists ' environmental quality perception. In Proceeding Euronoise 2015; Maastrícht, 2015; pp. 15351540 .

31. Liu, A.; Liu, F.; Deng, Z.; Chen, W. Relationship between soundscape and historical-cultural elements of Historical Areas in Beijing: A case study of Qianmen Avenue. In Proceeding Internoise 2014 - 43rd International Congress on Noise Control Engineering: Improving the World Through Noise Control; Melbourne, 2014; pp. 1-7.

32. Otto, N.; Amman, S.; Eaton, C.; Lake, S. Guidelines for Jury Evaluations of Automotive Sounds; 1999; Vol. April;

33. Lyon, R.H. Product sound quality: From perception to design. Sound Vib. 2003, 37, 18-23, doi:10.1121/1.4743110.

34. Blauert, J.; Jekosch, U. Sound-quality evaluation - A multi-layered problem. Acta Acust. united with Acust. 1997, 83, 747-753.

35. Fiebig, A. Influence of context effects on sound quality assessments. Euronoise 2015, 2555-2560.

36. Nykänen, A. Methods for Product Sound Design, Luleå University of Technology, 2008, Vol. Dr.

37. Blauert, J.; Jekosh, U. Auditory quality of performance spaces for music: The problem of the references. In ICA 200\%; Madrid, 2007; pp. 2-7.

38. Blauert, J. Analysis and Synthesis of Auditory Scenes. In Communication Acoustics; Blauert, J., Ed.; Springer, 2005; pp. 1-20 ISBN 3-540-22162-X.

39. Jekosch, U. Assigning Meaning to Sounds - Semiotics in the Context of 
Product-Sound Design. In Acoustics comunication; Blauert, J., Ed.; Springer: Berlin, 2005; pp. 193-219 ISBN 3-540-22162-X.

40. Santaella, L. Matrizes da Linguagem e o Pensamento; Iluminarias: São Paulo, 2013;

41. Peirce, C.S. Semiótica; 4ª ; Perspectiva: São Paulo, 2015; ISBN 9788527301947.

42. Santos, F.A. dos Design: a conexão do corpo com o ambiente e a sintaxe do pensamento humano. Triades 2010, 1, 1-18.

43. Santos, F.A. dos Comunicação visual e design como índice da complexidade semiótica do espaço urbano. In Urbanidades: mediações; Santos, F.A. dos, Camara, R., Eds.; Estereográfica Editorial: Brasilia, 2017; pp. 45-68.

44. Saleh, M.A.E. Place identity: The visual image of Saudi Arabian cities. Habitat Int. 1998, 22, 149-164, doi:10.1016/S0197-3975(97)00033-7.

45. Wagner, A. French Urban Space Management: A Visual Semiotic Approach Behind Power and Control. Int. J. Semiot. Law 2011, 24, 227-241, doi:10.1007/s11196-010-9206-5.

46. Otto, N.; Amman, S.; Eaton, C.; Lake, S. Guidelines for Jury Evaluations of Automotive Sounds. Sound Vib. 2001, April, 1-14, doi:1999-01-1822.

47. Otto, N.C. Listening test methods for automotive sound quality. In Audio engineering Society; Audio engineering Society: New York, 1997.

48. Lyon, R.H. Product sound quality: From perception to design. Sound Vib. 2003, 108, 2471, doi:10.1121/1.4743110.

49. Hinton, P.R.; McMurray, I.; Brownlow, C. Statistics Explained, 2nd Edition; 2014; ISBN 1317753119.

50. Axelsson, Ö.; Nilsson, M.E.; Berglund, B. A principal components model of soundscape perception. J. Acoust. Soc. Am. 2010, 128, 2836-2846, doi:10.1121/1.3493436.

51. Jeon, J.Y.; Lee, P.J.; You, J.; Kang, J. Perceptual assessment of quality of urban soundscapes with combined noise sources and water sounds. $J$. Acoust. Soc. Am. 2010, 127, 1357-1366, doi:10.1121/1.3298437.

52. Maristany, A.; Recuero López, M.; Asencio Rivera, C. Soundscape quality analysis by fuzzy logic: A field study in Cordoba, Argentina. Appl. Acoust. 2016, 111, 106-115, doi:10.1016/j.apacoust.2016.04.013.

53. Rey Gozalo, G.; Trujillo Carmona, J.; Barrigón Morillas, J.M.; VílchezGómez, R.; Gómez Escobar, V. Relationship between objective acoustic indices and subjective assessments for the quality of soundscapes. Appl. A coust. 2015, 97, 1-10, doi:10.1016/j.apacoust.2015.03.020.

54. Ricciardi, P.; Delaitre, P.; Lavandier, C.; Torchia, F.; Aumond, P. Sound quality indicators for urban places in Paris cross-validated by Milan data. 
J. Acoust. Soc. Am. 2015, 138, 2337-2348, doi:10.1121/1.4929747.

55. Ferrara, L.D. A estratégia dos signos: linguagem, espaço, ambiente urbano.; Segunda.; Perspectiva: San Pablo, 2009; ISBN 8527308398.

56. Tuan, Y.-F. Space and Place: The Perspective of Experience; 25Th ed.; University of Minnesota Press: Minneapolis, 1977; ISBN 0816638772.

57. Auge, M. Los no lugares. Espacios del anonimato.; Gedisa: Barcelona, 2009; ISBN 9788474324594.

58. Santos, F.A. dos Urbanidades mediações-Apresentação. In Urbanidades: mediações; Santos, F.A. dos, Camara, R., Eds.; Estereográfica Editorial: Brasilia, 2017; pp. 7-10.

59. Kang, J.; Aletta, F.; Gjestland, T.T.; Brown, L.A.; Botteldooren, D.; Schulte-fortkamp, B.; Lercher, P.; Kamp, I. Van; Genuit, K.; Luis, J.; et al. Ten questions on the soundscapes of the built environment. Build. Environ. 2016, 1-11, doi:10.1016/j.buildenv.2016.08.011.

60. Bento Coelho, J.L. Approaches to Urban Soundscape management, planning and design. In Soundscape and the Built Environment; Kang, J., Schulte-Fortkamp, B., Eds.; CRC Press, 2016; pp. 197-214.

61. Hermida, L.; Pavón, I.; Lobo Soares, A.; Bento-Coelho, J. On the PersonPlace Interaction and Its Relationship with the Responses/Outcomes of Listeners of Urban Soundscape (Compared Cases of Lisbon and Bogotá): Contextual and Semiotic Aspects. Int. J. Environ. Res. Public Health 2019, 16, 1-21, doi:10.3390/ijerph16040551. 


\section{CAPÍTULO 4}

\section{CONSIDERACIONES FINALES, CONCLUSIONES Y TRABAJOS A FUTURO.}




\section{CONSIDERACIONES FINALES, CONCLUSIONES Y TRABAJOS A FUTURO.}

\subsection{Consideraciones finales y conclusiones.}

Aunque el concepto de paisaje sonoro tiene sus principios en el campo de la música y el arte, en los últimos años este concepto se ha expandido al campo de la acústica ambiental. Su principal valor radica en que, además de analizar aspectos objetivos de los entornos acústicos, sitúa como eje central al ser humano y busca mejorar la calidad de vida de las personas. Este enfoque marca como gran desafío diseñar y/o gestionar ambientes acústicos que no solo sean no nocivos para la salud, sino también coherentes entre los diferentes estímulos ofrecidos en los lugares urbanos y con el uso que dan a los mismos las personas.

Por lo anterior, debido a que es un concepto aún en etapa de maduración, se hace necesario generar procesos y modelos que clarifiquen métodos de evaluación de paisajes sonoros y procesos de gestión acústica ambiental. En este sentido, las normas ISO 12913-1 e ISO 12913-2 son un buen punto de inicio en este proceso complejo ${ }^{3}$, aunque deben seguirse especificando diferentes aspectos relacionados con la recolección, procesamiento y uso de información, por lo que aún queda un largo camino por recorrer en este campo.

Teniendo en cuenta el marco conceptual propuesto por la norma ISO 12913-1, donde no existe paisaje sonoro sin entorno acústico, y que tampoco existe paisaje sonoro sin percepción, esta tesis doctoral vinculó tanto aspectos objetivos como subjetivos a fin de lograr su caracterización y conocimiento. Los aspectos más relevantes derivados de esta tesis se enuncian a continuación.

Si bien son conocidas las dificultades existentes para relacionar aspectos objetivos y subjetivos en la acústica ambiental, durante el desarrollo de esta tesis doctoral se exploraron herramientas descriptivas, parámetros objetivos y respuestas y salidas de corto y largo plazo de aplicación en paisajes sonoros

\footnotetext{
3 Para esta tesis doctoral lo complejo no se relaciona con la "dificultad" para desarrollar un proceso. Se adopta el concepto de pensamiento complejo de Edgar Morin, donde la complejidad hace referencia a la articulación entre dominios disciplinarios, por lo que el conocimiento adquiere un carácter multidimensional (Morin, 1990).
} 
urbanos. Los resultados presentados tanto en el segundo como en el tercer capítulo demuestran que la interacción entre varias disciplinas es posible, y que es indispensable que tanto parámetros acústicos como aspectos descriptivos y respuestas y salidas sean analizados en los procesos de evaluación de paisajes sonoros. En este sentido, uno de los principales aportes de esta tesis doctoral radica en la búsqueda exhaustiva de parámetros acústicos binaurales y temporales que permitieran relacionar aspectos descriptivos, objetivos y subjetivos.

Como se presentó en el segundo capítulo, debido a la gran variedad de atributos perceptuales que se relacionan con las respuestas a corto plazo en los paisajes sonoros, los parámetros acústicos no solo deben servir para describir la cantidad de energía existente en el entorno acústico, sino también para representar aspectos como variabilidad, uniformidad, o monotonía, donde promediados energéticos no son eficientes. En este campo es donde parámetros asociados a las funciones móviles de autocorrelación y correlación cruzada interaural (tiempo efectivo de duración $\left(\tau_{\mathrm{e}}\right)$ y el tiempo de IACC $\left(\tau_{\text {IACC }}\right)$ ) mostraron su utilidad en la evaluación y descripción de paisajes sonoros urbanos. Algunos de estos parámetros también mostraron asociaciones estadísticamente significativas no solo con atributos perceptuales, sino también con aspectos descriptivos como los sonidos fundamentales de los lugares en estudio.

La aplicación de parámetros binaurales en el estudio de paisajes sonoros permitió evidenciar que los aspectos relacionados con la localización espacial de las fuentes sonoras y las variaciones temporales impactan en la respuesta de los oyentes y en la morfología y características descriptivas de los paisajes sonoros.

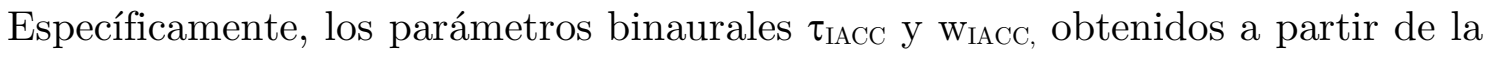
IACF móvil, permiten apreciar las variaciones que presentan las fuentes sonoras respecto a su localización espacial en el plano horizontal, mostrando además altos grados de correlación con distintos atributos perceptuales como Agitado, Calmo y Molesto, así como con los componentes principales Eventfulness y Pleasantness. Estadísticos descriptivos como el rango, o la diferencia entre percentiles 90-10 son indicadores que aportan significativamente en el estudio espacial de paisajes sonoros. En este sentido, los resultados obtenidos en esta tesis permiten apreciar que la agitación, la variabilidad, el caos o la uniformidad de los entornos acústicos 
pueden ser percibidos a partir de las variaciones de la ubicación espacial en el plano horizontal de las fuentes sonoras.

Así mismo, el parámetro $\tau_{\mathrm{IACC}}$ presentó diferencias estadísticamente significativas según el sonido fundamental de cada entorno acústico. Esto implica que los parámetros binaurales no solo son útiles en la evaluación de paisajes sonoros, sino que también permiten analizar aspectos descriptivos de los entornos acústicos.

En relación al parámetro $\tau_{\mathrm{e}}$, derivado de la ACF móvil, se encontró que también existen correlaciones entre aspectos objetivos y subjetivos. El rango de $\tau_{\text {e }}$ presentó una alta correlación negativa con el parámetro Monótono, así como correlaciones positivas moderadas con los atributos Agitado y el componente principal Eventfulness. Esto puede significar que algunas de las respuestas de corto plazo relacionadas con la variabilidad de las fuentes sonoras, pueden ser descritas con parámetros acústicos especializados en el estudio de la autosemejanza de señales.

Con base a lo anterior se concluye que la descripción y análisis de entornos acústicos debe hacerse no solo teniendo en cuenta aspectos energéticos, sino que también deben incluirse aspectos descriptivos, así como la variabilidad temporal y espacial de las fuentes sonoras en el plano horizontal. Este análisis, en conjunción con aspectos perceptuales, permitirá un abordaje amplio de los paisajes sonoros. De esta forma se da respuesta a la primera pregunta planteada en el primer capítulo, relacionada con ¿cómo evaluar paisajes sonoros, de tal forma que se obtenga información temporal y espacial que permita un diálogo entre aspectos objetivos y subjetivos?

Pasando a la segunda pregunta que motivó esta tesis, relacionada con la influencia del contexto en la evaluación de los paisajes sonoros según el conocimiento y experiencia previa de los oyentes en los lugares y según el tipo de metodología usada en el proceso de evaluación, fueron desarrolladas pruebas para comparar las metodologías de evaluación subjetiva in situ y en laboratorio y además se determinó la importancia de la interacción lugar-persona en las respuestas a corto plazo y en la salidas a largo plazo. En lo que respecta a las metodologías in situ y laboratorio, se encontraron diferencias significativas en las evaluaciones de paisajes sonoros según estos dos métodos de evaluación subjetiva. 
Estas diferencias se vieron manifestadas principalmente en los atributos Caótico (más caótico en laboratorio que in situ), Agitado (más agitado en laboratorio que in situ) y Uniforme/sin eventos (más uniforme in situ que en laboratorio), generando un impacto considerable en los componentes principales Pleasantness y Eventfulness del modelo de atributos perceptuales propuesto por Axelsson. Estas diferencias se presentan debido a dos razones principales: la existencia de diversos estímulos alternativos en las evaluaciones in situ (visuales, olfativos, táctiles y gustativos) que influencian la percepción del entorno y la variabilidad de la composición de los paisajes sonoros en las pruebas in situ respecto a las grabaciones presentadas en las pruebas en laboratorio.

Las dos metodologías de evaluación presentan sus ventajas y desventajas, por lo que sus utilidades y aplicaciones varían según el caso. Las evaluaciones in situ son importantes para el estudio holístico de los paisajes sonoros, donde además de los estímulos sonoros, el ambiente ofrece estímulos olfativos, táctiles y visuales, generando por ende una inmersión total en el ambiente. Las pruebas en laboratorio son útiles en evaluaciones de eventos sonoros específicos, permiten apreciar mayores detalles de los entornos acústicos, y además desarrollar procesos de comparación, simulación y diseño de paisajes sonoros.

En referencia a la interacción lugar-persona y su influencia en las respuestas a corto plazo (atributos perceptuales relacionados con las actitudes de las personas) y las salidas a largo plazo (usos y tiempos de permanencia referidos a los comportamientos de las personas) se encontró que, utilizando la metodología de pruebas en laboratorio, las respuestas a corto plazo son poco dependientes del lugar de origen de los evaluadores, mientras que para salidas a largo plazo si existe esta dependencia. Estos resultados indican que, bajo las condiciones de las pruebas realizadas, la interacción lugar-persona (experiencias previas) genera poco impacto en la percepción de atributos de los paisajes sonoros, aunque si es importante en las salidas a largo plazo.

Para analizar los resultados encontrados respecto a las salidas a largo plazo, en esta investigación fueron de gran utilidad algunas teorías del campo de la comunicación y la semiótica, donde los significados de los lugares se construyen según el uso que dan las personas al lugar. En este sentido, dado que las personas que tenían experiencias previas en los lugares en estudio ya tenían conocimiento 
respecto a los posibles usos (por ende, conocen su significado), se encuentra una explicación clara en la diferencia entre las salidas a largo plazo según el lugar de procedencia del evaluador: quienes tenían una interacción con los lugares conocen su significado, lo que proporciona un contexto previo. La interacción lugarpersona es producto de la experimentación directa de la persona, generando memorias y experiencias previas que alimentan las expectativas y las actividades a desarrollarse en el lugar. Bajo condiciones de laboratorio y sin estas experiencias previas, los oyentes asociarán los entornos acústicos a sus propias experiencias con lugares de características diferentes, lo que generará una diferencia de significados según el lugar de procedencia del oyente.

Así mismo, durante el análisis de resultados obtenidos en el estudio comparativo de evaluaciones subjetivas según el lugar de procedencia del oyente, fueron encontrados algunos lugares cuyas respuestas a corto plazo y salidas a largo plazo no variaban según el origen de los evaluadores. Avenidas o calles simples, pueden ser considerados espacios no representativos y, dado que su uso es similar en cualquier parte, su significado también lo es. El antropólogo Marc Augé define este tipo de espacios como los "no lugares", y dado que, según los resultados encontrados, el fenómeno parece replicarse en los paisajes sonoros urbanos, esta tesis se propone utilizar el término no lugares sonoros, en referencia a aquellos entornos acústicos sin identidad ni significado.

Los hallazgos presentados en el Capítulo 3 de esta tesis permiten a su vez establecer vínculos entre aspectos contextuales y semióticos con procesos de gestión y diseño de paisajes sonoros urbanos. En esta línea, para una mejor comprensión de los paisajes sonoros urbanos, se adaptó un modelo de semiosis proveniente del campo de la calidad acústica, estableciendo una relación entre el lugar, el entorno acústico y el paisaje sonoro, teniendo como eje central a la persona. A partir de este modelo de semiosis (claramente permeado por aspectos contextuales) así como de la interacción lugar-persona (y su influencia en el significado), se plantearon diferentes cuestionamientos relacionados con la necesidad de diseñar y gestionar entornos acústicos coherentes con el objetivo, significado y uso de los lugares. Esto implica que los procesos de gestión y diseño acústico ambiental requiere de enfoques interdisciplinares que fortalezcan el uso y el significado de los espacios urbanos. 
Las dos preguntas de investigación planteadas al inicio de esta tesis motivaron y permitieron la interacción de diferentes campos del conocimiento, teniendo que transitar entre parámetros acústicos binaurales y temporales, así como por teorías de percepción y diferentes aspectos subjetivos de evaluación de entornos ambientales en la búsqueda de posibles respuestas. Fueron detectados puntos de encuentro entre estos dos enfoques, donde por una parte los parámetros acústicos permitieron el entendimiento de aspectos descriptivos y subjetivos y por otra se encontraron aspectos subjetivos que permiten no solo esclarecer la influencia de algunos componentes contextuales en la evaluación de paisajes sonoros, sino que además son potencialmente útiles en la gestión acústica ambiental.

Por otra parte, si bien esta tesis desarrolló experimentos in situ, la gran mayoría de pruebas fueron realizadas en laboratorio. La posibilidad de reducir las variables que influencian las salidas y respuestas de los oyentes, el control sobre eventos sonoros específicos a presentar a los evaluadores, así como la gran utilidad en etapas de diseño y simulación, hacen de las pruebas de laboratorio una potente herramienta en la evaluación y gestión acústica ambiental. Además, aunque la norma ISO 12913-1 indica en su nota 1 que los entornos acústicos pueden ser reales o simulados, ésta y la norma ISO 12913-2 (sobre metodología de recolección de datos de paisajes sonoros) se enfocan principalmente en procesos de evaluación in situ. Como se ha mencionado a lo largo de este documento, estos dos métodos pueden tener diferentes usos y aplicaciones, dado que las variables que influyen en la evaluación de paisajes sonoros son también diferentes. Por todos estos motivos, se considera que, así como existen normas de evaluación de barreras acústicas o de silenciadores in situ y en laboratorio (por mencionar dos ejemplos), es necesario que se desarrollen modelos que permitan diferenciar métodos de evaluación de paisajes sonoros in situ y en laboratorio según las diferentes aplicaciones y necesidades.

Por tal razón, esta tesis presenta mediante la figura 4-1 lo que podría ser considerado un primer acercamiento al modelo de evaluación de paisajes sonoros en laboratorio. Dicho modelo contiene aspectos descriptivos y objetivos de entornos acústicos, así como su relación con respuestas y salidas en paisajes sonoros. 


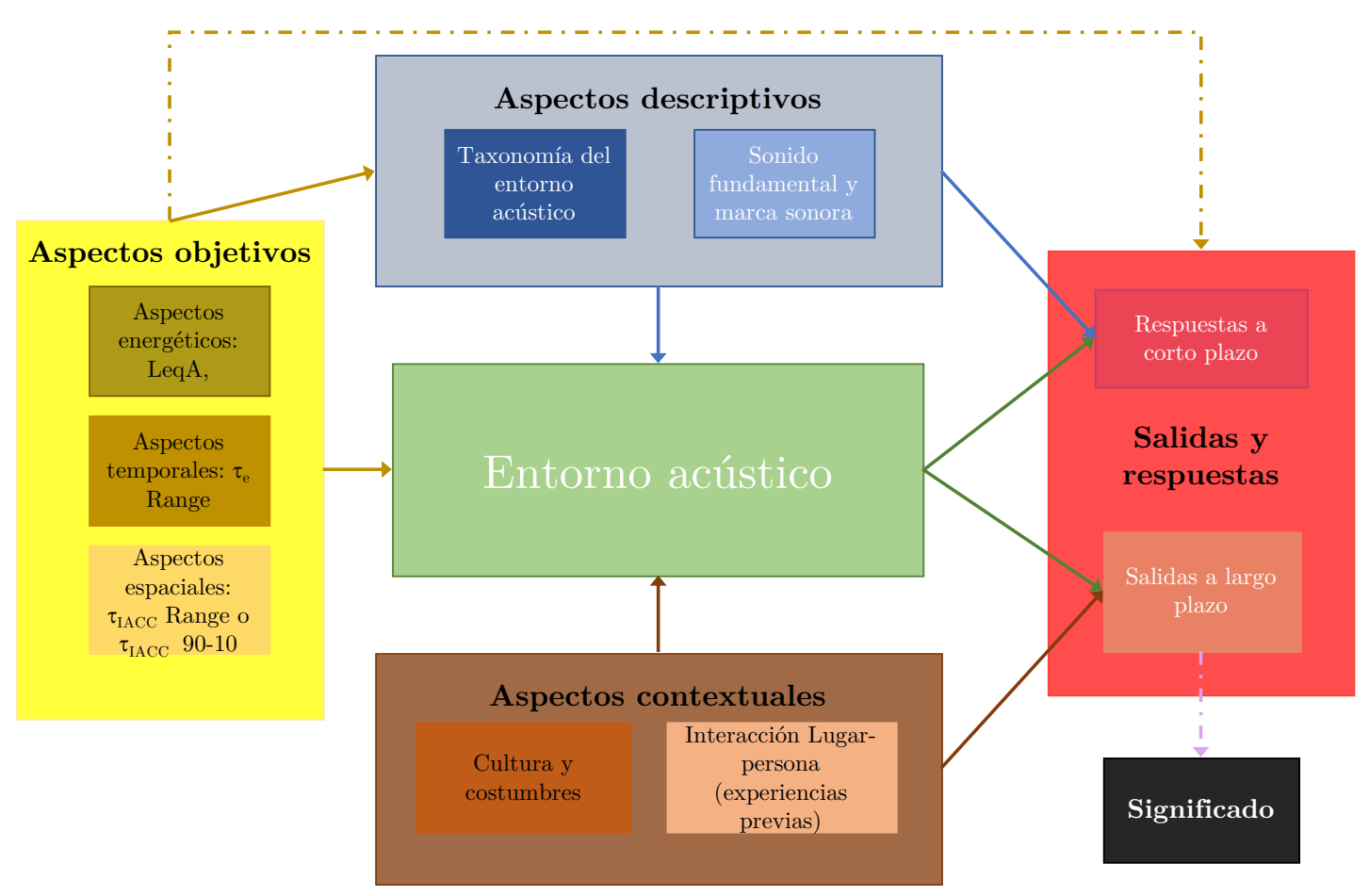

Figura 4-1. Modelo de evaluación subjetiva de paisajes sonoros en laboratorios.

El modelo de evaluación de paisajes sonoros en laboratorio propuesto en esta tesis se fundamenta en el marco conceptual de la norma ISO 12913-1 y está constituido por cuatro grandes componentes: Aspectos objetivos, Aspectos descriptivos, Aspectos contextuales y Respuestas y salidas.

Respecto a Aspectos objetivos, el modelo de evaluación incluye el estudio del comportamiento energético debido a la necesidad de analizar los niveles de emisión acústica y su impacto en la salud y el confort, pero además se incluyen parámetros temporales y espaciales, debido a que con ellos es posible obtener información relacionada con aspectos descriptivos y presentan correlaciones considerables con las respuestas a corto plazo (diferentes atributos perceptuales).

En lo que respecta a Aspectos descriptivos, según los resultados de esta tesis, se considera importante incluir herramientas como clasificación de fuentes sonoras y sonidos existentes en el entorno acústico en los procesos de evaluación de paisajes sonoros en laboratorio. Esta información permite determinar la composición del entorno acústico, así como las actividades desarrolladas en los ambientes en estudio. Tanto los Aspectos objetivos como los Aspectos descriptivos contribuyen a un análisis amplio de los entornos acústicos. 
En relación a los Aspectos contextuales, el modelo presentado se enfoca en evaluaciones en laboratorio, por lo que no son tenidos en cuenta elementos contextuales del lugar (estímulos olfativos, visuales y táctiles), aunque si se incluyen las experiencias previas del oyente dadas por la interacción del lugarpersona, así como aspectos culturales (uso de lugares según prácticas y cultura).

Finalmente, respecto a las Salidas y Respuestas, se considera que a fin de determinar tanto la percepción a corto plazo como también respuestas a largo plazo, las evaluaciones deben realizarse teniendo en cuenta diversos atributos perceptuales (agradable, desagradable, uniforme, etc.), así como usos y tiempos de permanencia en lugares con entornos acústicos como los evaluados. Según los resultados presentados en el tercer capítulo, de las salidas a largo plazo puede inferirse el significado de los lugares en estudio según el contexto, por lo que en este modelo no se relacionan las respuestas a corto plazo con las salidas a largo plazo.

Para la aplicación del modelo de evaluación propuesto en esta tesis se consideran útiles las herramientas desarrolladas a lo largo de esta investigación presentadas en los anexos 2 y 5 , aunque deben ajustarse no solo al idioma del país de aplicación, sino también a los modismos lingüísticos típicos.

\subsection{Líneas futuras de investigación.}

Teniendo en cuenta el desarrollo de esta tesis doctoral, se considera imprescindible consolidar grupos de investigación que aborden la temática de evaluación y diseño de paisajes sonoros de forma interdisciplinar. Ingenieros, arquitectos, psicólogos, comunicadores, diseñadores, urbanistas, antropólogos y artistas pueden (y deben) interactuar para la construcción de paisajes sonoros acordes al contexto y cultura de cada lugar.

Pasando a investigaciones mucho más específicas, se proponen dos grandes líneas: evaluación de paisajes sonoros y diseño y gestión de paisajes sonoros.

Respecto a la evaluación de paisajes sonoros, deben seguirse desarrollando investigaciones que permitan establecer la existencia de correlaciones entre parámetros objetivos y salidas a largo plazo. Así mismo se considera necesario seguir fortaleciendo procesos de clasificación de entornos acústicos según su 
taxonomía, donde de forma objetiva y subjetiva pueda determinarse tanto marcas sonoras como sonidos fundamentales que componen los entornos sonoros. En este sentido, también se considera necesario desarrollar investigaciones en donde se determine la influencia de marcas sonoras y sonidos fundamentales en las salidas a largo plazo.

También pueden desarrollarse proyectos de investigación que permitan determinar la influencia de los diferentes componentes del modelo de evaluación contextual en la evaluación de paisajes sonoros, siendo necesario trabajar en la importancia del componente contextual Persona en estos procesos. Es importante que en las pruebas de evaluación subjetiva en laboratorio se empiecen a generar mayores niveles de inmersión en los ambientes en estudio, por lo que el uso de tecnologías de realidad virtual puede ser de gran ayuda en estos procesos. Se considera necesaria la normalización de procesos de evaluación de paisajes sonoros, marcando una clara diferencia entre metodologías in situ y en laboratorio según las necesidades y aplicaciones requeridas.

En lo relativo a investigaciones en diseño y gestión de paisajes sonoros, como fue mencionado anteriormente, el acercamiento entre áreas del conocimiento como el diseño, la ingeniería y la arquitectura debe hacerse más fuertes y recurrentes. Diseñar espacios coherentes, que tengan en cuenta una oferta sonora (el término Affordance sonoro fue propuesto en el tercer capítulo de esta tesis) acorde al uso y contexto, debe convertirse en una práctica en la gestión acústica ambiental. Por supuesto es necesario asegurar que la salud de los habitantes de las ciudades no está en riesgo, pero además deben generarse procesos de interacción entre diferentes áreas del conocimiento para que las personas no solo encuentren confortable o no molesto su entorno acústico, sino que además generen experiencias sonoras urbanas en las personas.

El desarrollo de políticas claras de gestión acústica ambiental bajo el enfoque de paisajes sonoros, no solo asegurarán la protección de la salud de los habitantes de las ciudades, sino que también reforzarán la identidad de las personas y mejorarán la calidad de vida de las personas. Sin embargo, antes de llegar a este punto es necesario estandarizar procesos de evaluación y ofrecer pautas generales para la gestión acústica ambiental, siempre pensando que es el usuario de la ciudad quien se verá beneficiado por estos enfoques. 


\section{CHAPTER 4}

\section{FINAL CONSIDERATIONS AND CONCLUSIONS (ENGLISH VERSION)}




\section{FINAL CONSIDERATIONS CONCLUSIONS.}

\subsection{Final considerations and conclusions.}

Although the concept of soundscape has its origins in music and art fields, in recent years this concept has expanded to environmental acoustics. Its main value is that, in addition to analyzing objective aspects of acoustic environments, the human being as its central axis and tends to improve people's quality of life. This approach makes it a great challenge to design and/or manage acoustic environments that are not only harmful to health, but also coherent between the different stimuli offered in urban places and the use given to them by people.

Therefore, considering that the concept of soundscape is still being developed, it is necessary to generate processes and models that clarify assessment methods and environmental acoustic management processes. In this sense, the norms ISO 12913-1 and ISO 12913-2 are a start point in this complex ${ }^{4}$ evaluation process, although different aspects related to the collection, processing, and use of information must continue to be specified, so there is still a long way to go in this field.

Given the conceptual framework proposed by ISO 12913-1, where there is no soundscape without an acoustic environment, and that there is no soundscape without perception, this thesis linked both objective and subjective aspects to achieve their characterization and knowledge. The most relevant aspects derived from this thesis are listed below.

Although the difficulties to relate objective and subjective aspects in environmental acoustics are known, during the development of this doctoral thesis where explored descriptive tools, objective parameters, and responses and outcomes of short and long term of application in urban soundscapes. The results

\footnotetext{
${ }^{4}$ For this doctoral thesis complexity it is not related to the "difficulty" to develop a process. The concept of complex thinking is adopted by Edgar Morin, where complexity refers to the articulation between disciplinary domains, so that knowledge acquires a multidimensional character (Morin, 1990).
} 
presented in both the second and third chapters demonstrate that the interaction between various disciplines is possible and that it is essential the analysis in the processes of assessment of soundscapes of both acoustic parameters and descriptive aspects and responses and outcomes. In this sense, one of the main contributions of this doctoral thesis lies in the exhaustive search of acoustic binaural and temporal parameters that allow relating descriptive, objective and subjective aspects.

As it is presented in the second chapter, because of the wide variety of perceptual attributes that relate to short-term responses in soundscapes, acoustic parameters must not only serve to describe the amount of energy in the acoustic environment, but also to represent aspects such as variability, uniformity, or monotony, where energy averaging is not efficient. In this aspect, parameters associated to the autocorrelation and interaural cross-correlation functions (effective duration time $\left(\tau_{\mathrm{e}}\right)$ interaural cross-correlation (IACC) and IACC time $\left(\left(\tau_{\text {IACC }}\right)\right.$ were used in the evaluation and description of urban soundscapes. Some of these parameters also showed statistically significant associations not only with perceptual attributes, but also with descriptive aspects such as the keynote sounds of the places under study.

The application of binaural parameters in the soundscape's studies allowed to make evident that aspects related to the spatial location of sound sources and temporal variations impact the response from listeners and morphology and descriptive characteristics of the soundscapes. Specifically, the binaural parameters IACC, $\tau_{\mathrm{IACC}}$ and $\mathrm{w}_{\mathrm{IACC}}$, obtained from the running IACF, allow to appreciate the variations that present the sound sources concerning with their spatial location in the horizontal plane, showing also high correlation with different perceptual attributes such as Agitated, Calm and Annoying, as well as with the main components Eventfulness and Pleasantness. Descriptive statistics such as range, or the difference between 90-10 percentiles are indicators that contribute significantly in the spatial study of the soundscape. In this sense, the results obtained in this thesis allow to appreciate that the agitation, variability, chaos or uniformity of the acoustic environments can be perceived from the variations of sound sources' spatial location in the horizontal plane. 
Also, $\tau_{\text {IACC }}$ parameter presented statistically significant differences according to the keynote sound of each acoustic environment. This implies that binaural parameters are not only useful in the evaluation of the soundscape, but also allow the analysis of acoustic environments' descriptive aspects.

In relation to the $\tau_{\mathrm{e}}$ parameter derived from running $\mathrm{ACF}$, it was found that there are also correlations between objective and subjective aspects. The $\tau_{\mathrm{e}}$ range presented a high negative correlation with the Monotonous parameter, as well as moderate positive correlations with Agitated attribute and the main component Eventfulness. This means that some of the short-term responses related to the variability of sound sources can be described with specialized acoustic parameters in the study of signals self-similarity.

Based on the above, it is concluded that the description and analysis of acoustic environments should be done not only considering energy aspects, but also include descriptive aspects, as well as the temporal and spatial variability of sound sources in the horizontal plane. This analysis, in conjunction with perceptual aspects, will allow a broad approach to soundscapes. This is the answer to the first research question posed in the first chapter, related to how to evaluate soundscapes, in order to obtain temporal and spatial information that allows a dialogue between objective and subjective aspects?

In relation to the second research question that motivated this thesis, related to the context influence in the soundscapes assessment according to the listeners' previous knowledge and experience of the places and according to the type of methodology used in the evaluation process, tests were developed to compare the methods of subjective evaluation in situ and in laboratory. Also, the importance of the Person-Place Interaction in the short-term responses and in the long-term outcomes was determined. In terms of in situ and laboratory methods, significant differences were found in the evaluation of soundscape according to these two methods of subjective evaluation. These differences were manifested mainly in Chaotic (more chaotic in laboratory than in situ), Agitated (more agitated in laboratory than in situ) and Uniform/no events (more uniform in situ than in laboratory) attributes, generating a considerable impact in the main components Pleasantness and Eventfulness of the perceptual attributes model proposed by Axelsson. These differences arise due to two main reasons: the 
existence of various alternative stimuli in the in situ tests (visual, olfactory, tactile and gustatory) that influence the environment perception and the variability of the composition of the soundscape in the in Situ tests with respect to the recordings presented in the laboratory tests.

The two evaluation methods present their advantages and disadvantages, so that their utilities and applications vary according to the case. In situ assessments are important for the holistic study of soundscape, where in addition to sound stimuli, the environment offers olfactory, tactile and visual stimuli, thus generating a total immersion in the environment. Laboratory tests are useful in evaluating specific sound events, allowing greater appreciation of acoustic environments, and permitting the development of processes for comparison, simulation and design of soundscape.

About the Person-Place interaction and its influence on short-term responses (perceptual attributes related to people's attitudes) and long-term outcomes (uses and permanency times related to people's behaviour), it was found that, using the laboratory test method, short-term responses are slight dependent on the evaluators' origin place, while for long-term departures this dependency exists. These results indicate that, under the conditions of the tests performed, the Person-Place Interaction (previous experiences) has little impact on the perception of attributes of the soundscapes, although it is important in the longterm outcomes.

To analyze the results regarding long-term outcomes, in this research, theories from the field of communication and semiotics were useful, especially related with the construction of meaning according to the use that the people give to the place. In this sense, people with previous experiences in the places, already had knowledge about possible uses (hence knowing their meaning). There is a clear explanation in the difference between long-term outcomes according to the evaluators' place of origin: those who had a previous interaction with the places know their meaning, which provides a former context. The Person-Place Interaction is a product of the person's direct experimentation, which generates memories and previous experiences that feed the expectations and the activities to be developed in the place. Under laboratory conditions and without these previous experiences, listeners will associate the acoustic environments to their 
own experiences with places of different characteristics, which will generate a difference of meanings according to the listener place of origin.

Likewise, during the analysis of results generated in the comparative study of subjective evaluations according to the listener's place of origin, were found some places whose short-term responses and long-term outcomes did not vary according to the origin of the evaluators. Simple avenues or streets can be considered as non-representative spaces and, since their use is similar anywhere, it's meaning too. The anthropologist Marc Augé defines them as "no places", and according to the results found, the phenomenon seems to replicate in urban soundscapes, this thesis proposes to use the term "no sound places", referring to those acoustic environments without Identity and meaning.

The findings presented in Chapter 3 of this thesis allow in turn to establish links between contextual and semiotic aspects with management processes and design of urban soundscapes. In this line, for a better understanding of urban soundscapes, a semiosis model was adapted from the field of acoustic quality, establishing a relationship between the place, the acoustic environment and the soundscape, having the person as the central axis. From this model of semiosis (clearly permeated by contextual aspects) as well as the Person-Place Interaction (and its influence on meaning), different questions were raised related to the need to design and manage acoustic environments consistent with the objective, meaning and use of places. This implies that environmental acoustic design and management processes require interdisciplinary approaches that strengthen the use and meaning of urban spaces.

The two research questions posed at the beginning of this thesis motivated and enabled the interaction of different fields of knowledge, having to move between binaural and temporary acoustic parameters, as well as theories of perception and different subjective aspects of evaluation of environmental conditions in the search of possible answers. Points of encounter were detected between these two approaches, where on the one hand, the acoustic parameters allowed the understanding of descriptive and subjective aspects and on the other, subjective aspects were found that allows not only to clarify the influence of some contextual components soundscapes evaluation but are also potentially useful in acoustic environmental management. 
Moreover, although this thesis developed in situ experiments, most tests were performed in the laboratory. The possibility of reducing the variables that influence the listener's outcomes and responses, the control over specific sound events to be presented to the evaluators, as well as the great usefulness in design and simulation stages, make laboratory tests a powerful tool in environmental acoustic assessment and management. In addition, although ISO 12913-1 indicates in the note 1 that the acoustic environments may be current or simulated, this document and the ISO 12913-2 (on the methodology of soundscapes data collection) are mainly focused on in situ evaluation processes. As mentioned through this document, these two methods may have different uses and applications, since the variables that influence the soundscape evaluation are also different. For all these reasons, it is considered that, as there are standards for the evaluation of acoustic barriers or silencers in situ and in the laboratory (to mention two examples), it is necessary to develop models that allow to differentiate in situ and in laboratory soundscape evaluation methods according to the different applications and needs.

For these reasons, this thesis presents Figure 4-1 which could be considered a first approach to the soundscapes laboratory assessment model. This model contains descriptive and objective aspects of acoustic environments, as well as their relationship with responses and outcomes in soundscapes.

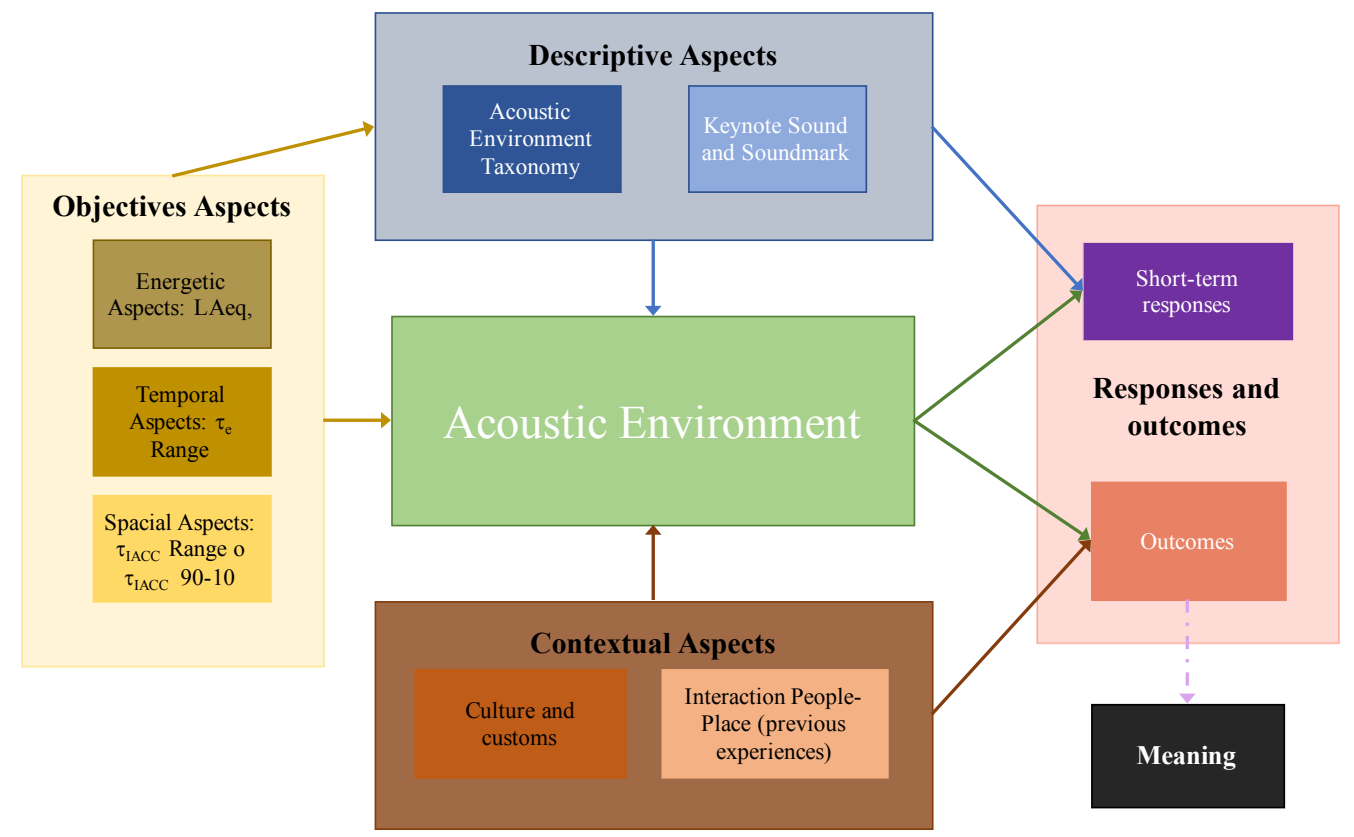

Figure 4-1. Subjective assessment model of soundscapes in laboratories. 
The soundscapes laboratory assessment model proposed in this thesis, presented in Figure 4-1, is based on the ISO 12913-1 conceptual framework and consists of four major components: Objective aspects, Descriptive aspects, Contextual aspects and Responses and Outcomes.

Regarding Objective aspects, the evaluation model includes the study of energy behavior due to the need to analyze the levels of acoustic emission and its impact on health and comfort, but also include temporal and spatial parameters, because with them it is possible to obtain information related to descriptive aspects and they show considerable correlations with short-term responses (different perceptual attributes).

Regarding Descriptive aspects, according to the results of this thesis, it is considered important to include tools such as sound sources classification and existing sounds in the acoustic environment in the soundscape assessment. This information allows to determine the composition of the acoustic environment, as well as the activities developed in the environments under study. Both the objective Aspects and the Descriptive Aspects contribute to a comprehensive analysis of acoustic environments.

In relation to the Contextual aspects, the model presented focuses on laboratory evaluations, so that contextual elements of the place (olfactory, visual and tactile stimuli) are not considered, although are included the previous experiences of the listener generated from interaction of Place-Person. Cultural aspects (use of places according to practices and culture) are included.

Finally, about Outcomes and Responses, it is considered that to determine both short-term perceptions and long-term responses, assessments should be made considering various perceptual attributes (pleasant, unpleasant, uniform, etc.) and the uses and times of permanence in places with acoustic environments like those evaluated. According to the results presented in the third chapter, the longterm outcomes can be inferred the meaning of the places under study according to the context, reason why in this model it is not related the short-term answers with the long-term outcomes.

For the application of the evaluation model proposed in this thesis, the tools developed through this research presented in annexes 2 and 5 are considered 
useful, although they must adjust not only to the country's language where occurs the application, but also to the typical linguistic idioms.

\subsection{Future research lines.}

Given the development of this thesis, it is considered essential to consolidate research groups to address the issue of evaluation and design of soundscapes in an interdisciplinary way. Engineers, architects, psychologists, communicators, designers, urbanists, anthropologists and artists can (and should) interact for the construction of soundscape according to the context and culture of each place.

Moving on more specific research, two major lines are proposed: soundscape evaluation and design and management of soundscapes.

Regarding the soundscape evaluation, research should continue to be carried out to establish the existing correlations between objective parameters and long-term outputs. Likewise, it is considered necessary to continue strengthening the classification processes of acoustic environments according to their taxonomy, where in an objective and subjective way can be determined by both soundmarks and keynote sounds that compose the sound environments. In this sense, it is also considered necessary to develop research in which the influence of sound marks and keynote sounds in the long-term outcomes is determined.

Other possible research projects may be related to determine the influence of the different components of the contextual evaluation model in the soundscape evaluation, being necessary to work on the importance of the contextual component Person in the evaluation process. It is important that subjective laboratory tests begin to generate higher levels of immersion in the study environments, so the use of virtual reality technologies can be of great help in these processes. It is considered necessary the standardization of soundscape evaluation processes, marking a clear difference between methodologies in situ and in the laboratory according to the needs and applications required. 
About research in design and management of soundscapes, as mentioned above, the conciliation between areas of knowledge such as design, engineering and architecture must become stronger and recurrent. Design congruent spaces, considering a sound affordance according to the use and context, should become a practice in environmental acoustic management. Of course, it is necessary to ensure that the health of the cities habitants is not at risk. In addition, processes of interaction between different fields of knowledge must be generated so that people not only find comfortable or not annoying their acoustic environment, but also should seek the generation of urban sound experiences in people.

The development of clear environmental acoustic management policies under the soundscape approach would not only ensure the protection of the health of city dwellers but would also reinforce the identity of individuals and improve the quality of life of people. Before reaching this point, however, it is necessary to standardize evaluation processes and offer general guidelines for acoustic environmental management, always thinking that it is the user of the city who will benefit from these approaches. 


\section{ANEXOS}




\section{Anexo 1. Presentación de lugares en estudio Bogotá y Brasil.}

1. Avenida Eixo Rodoviario (BRER): Esta avenida es una de las principales arterias vehiculares de la ciudad de Brasilia. Junto con avenida Eixo monumental dan forma al plano piloto de la ciudad. Con cerca de 13 kilómetros de extensión, esta avenida atraviesa la ciudad de norte a sur y está compuesta por un eje principal (denominado Eixao) y dos ejes secundarios a cada lado (eixinhos). El eje principal está compuesto por tres carriles por cada sentido y los ejes secundarios están compuestos por otros cuatro carriles por cada lado. El punto escogido para la grabación binaural fue la calle SQN 109 a 20 metros de la avenida en el sentido norte-sur, en un espacio abierto rodeado por edificaciones de máximo 6 pisos. La grabación se realizó paralelo al Eixo rodoviário (P1).

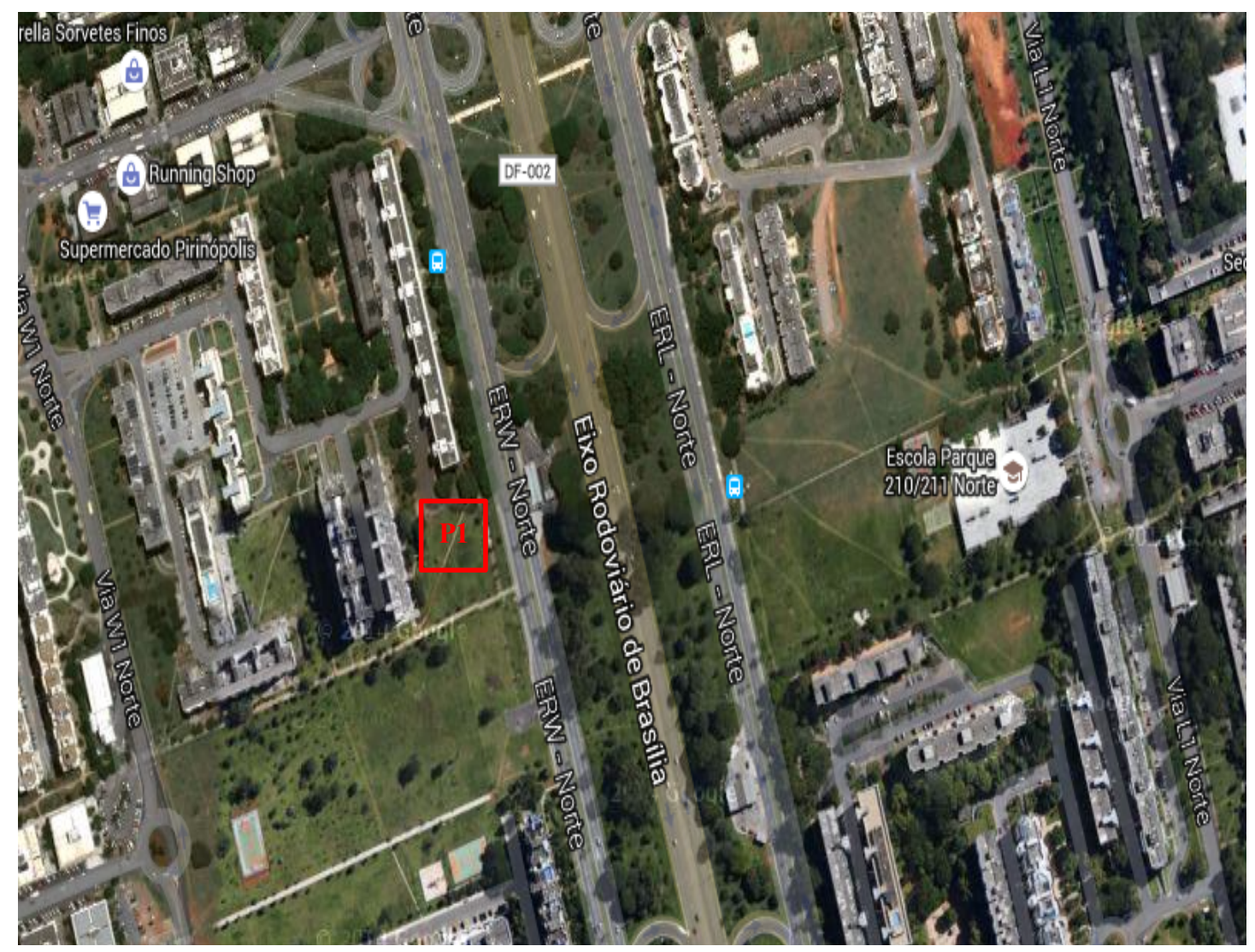

2. Feria torre de TV (FTV): La feria de artesanía permanente de la torre de TV está ubicada en el corazón de Brasilia en la avenida Eixo monumental. Es la feria en espacio abierto más antigua de Brasília, funcionando desde 1970. Este punto fue escogido por la diversidad de eventos sonoros presentes que van desde actividades comerciales, culturales y artísticas 
hasta de alimentación. Los días de mayor movimiento son los sábados entre las 11 y las 17 horas. La grabación binaural fue realizada en el centro de la fuente, paralelo al Eixo monumental (P2).

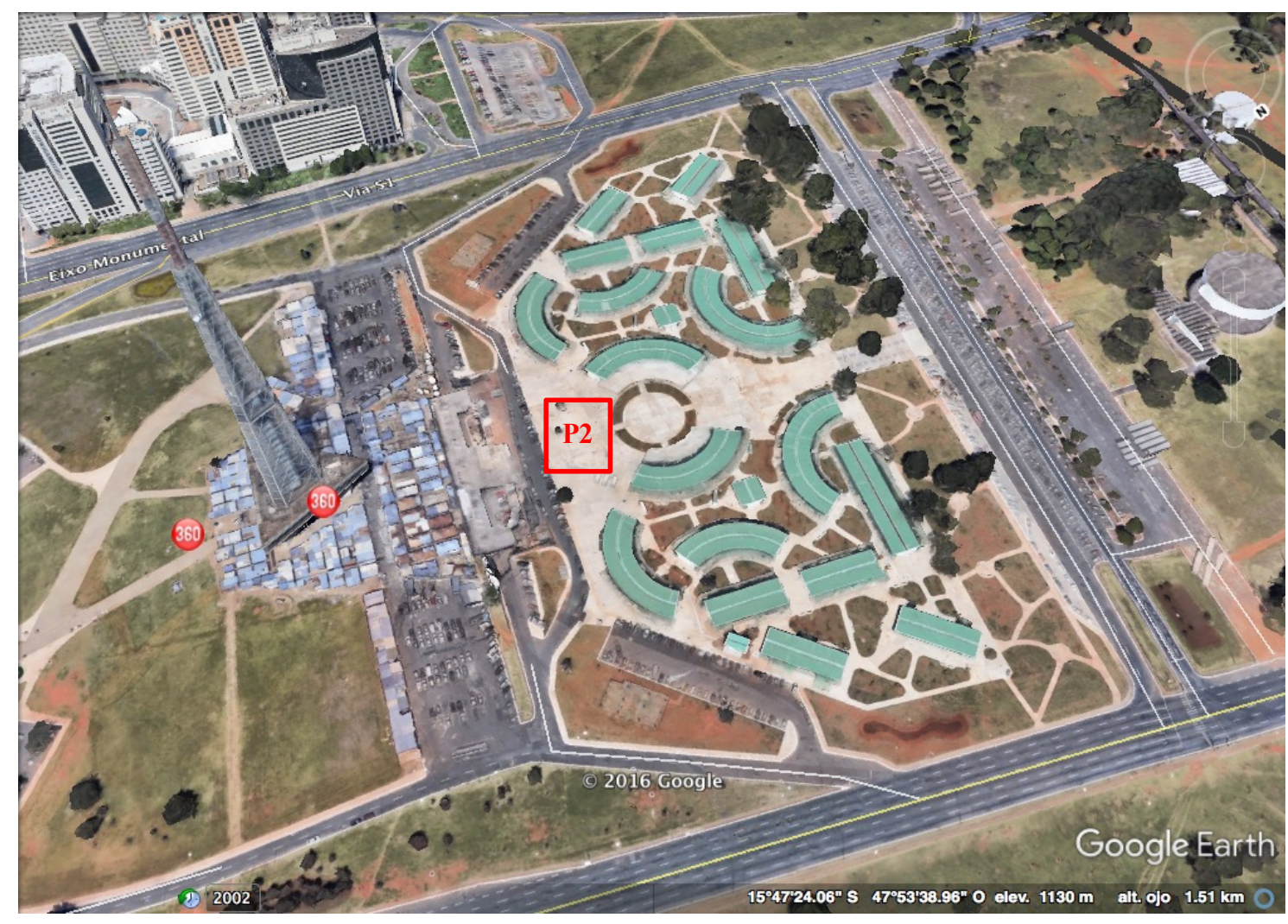

3. Fuente de agua (BRFA): También ubicada en la mitad de la avenida Eixo monumental, la fuente de agua escogida es la más grande de la ciudad y está ubicada a menos de 200 metros torre de TV. Debido a las altas temperaturas de Brasilia, que en épocas de verano pueden alcanzar los 38 ${ }^{\circ} \mathrm{C}$, es común encontrar personas descansando y compartiendo en familia, o disfrutando de actividades al aire libre y conciertos. La fuente tiene un diámetro de cerca de 80 metros y su caudal principal de agua puede alcanzar hasta cincuenta metros de altura. Al igual que en la feria torre de TV, se realizaron grabaciones en el plano paralelo al Eixo monumental, a cinco metros de la fuente de agua (P3). 


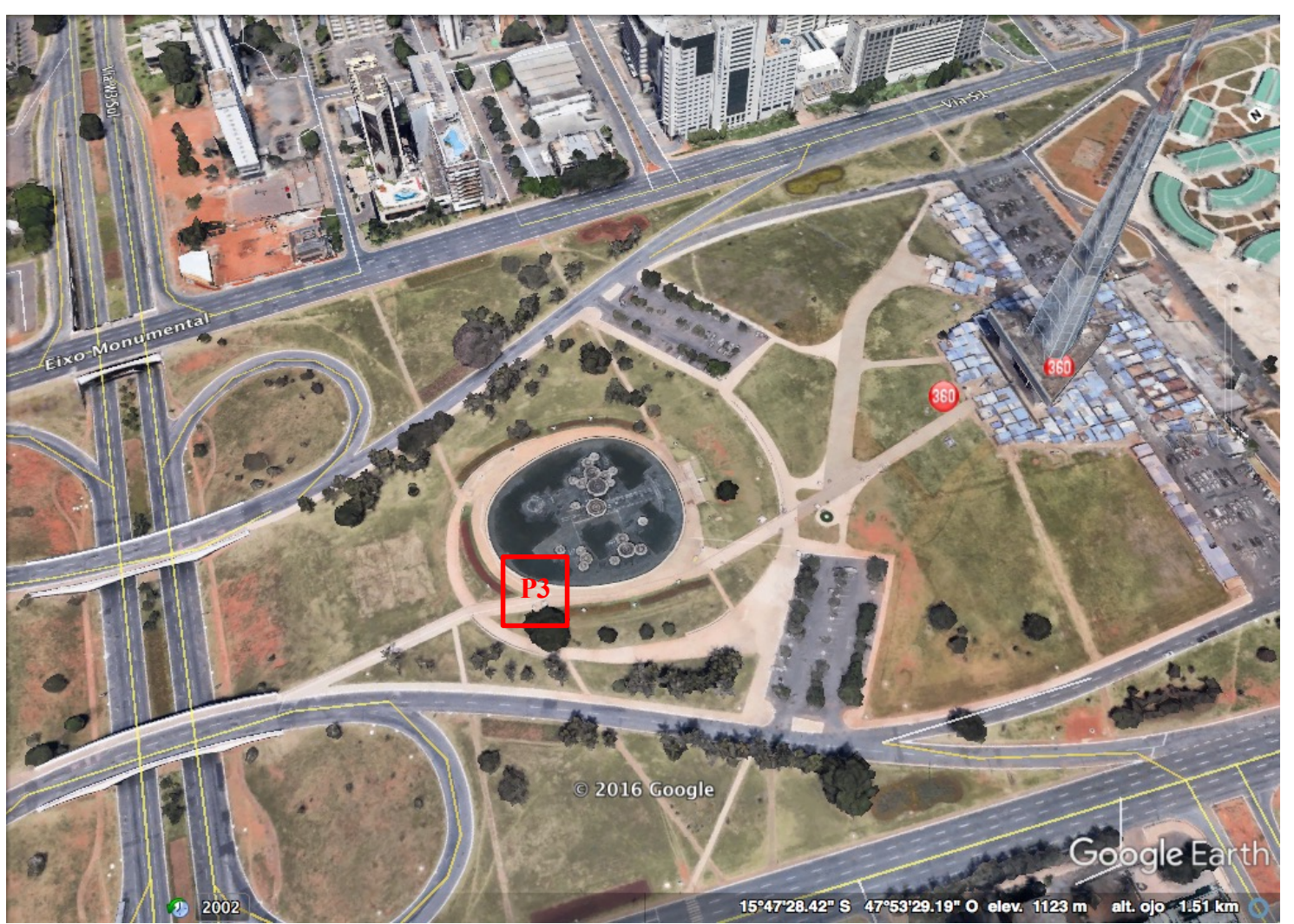

4. Parque Olhos D'agua (BRPOA): Este parque natural es frecuentemente visitado por personas del sector para hacer deportes y avistar pájaros. Fue inaugurado en el año 1994 y está ubicado en el sector norte de la ciudad, sobre la avenida L2 norte a la altura de la calle 413 norte. Cuenta con 28 hectáreas y está dotado de máquinas para ejercitarse físicamente, caminos y circuitos de caminata y un pequeño lago. La vegetación es típica de la región, conocida como "cerrado", caracterizada por su densidad y baja altura. Como en toda la ciudad de Brasília, las edificaciones cercanas al parque tienen una altura máxima permitida de seis pisos. Las grabaciones binaurales se realizaron paralelas a la vía L2N (P4). 


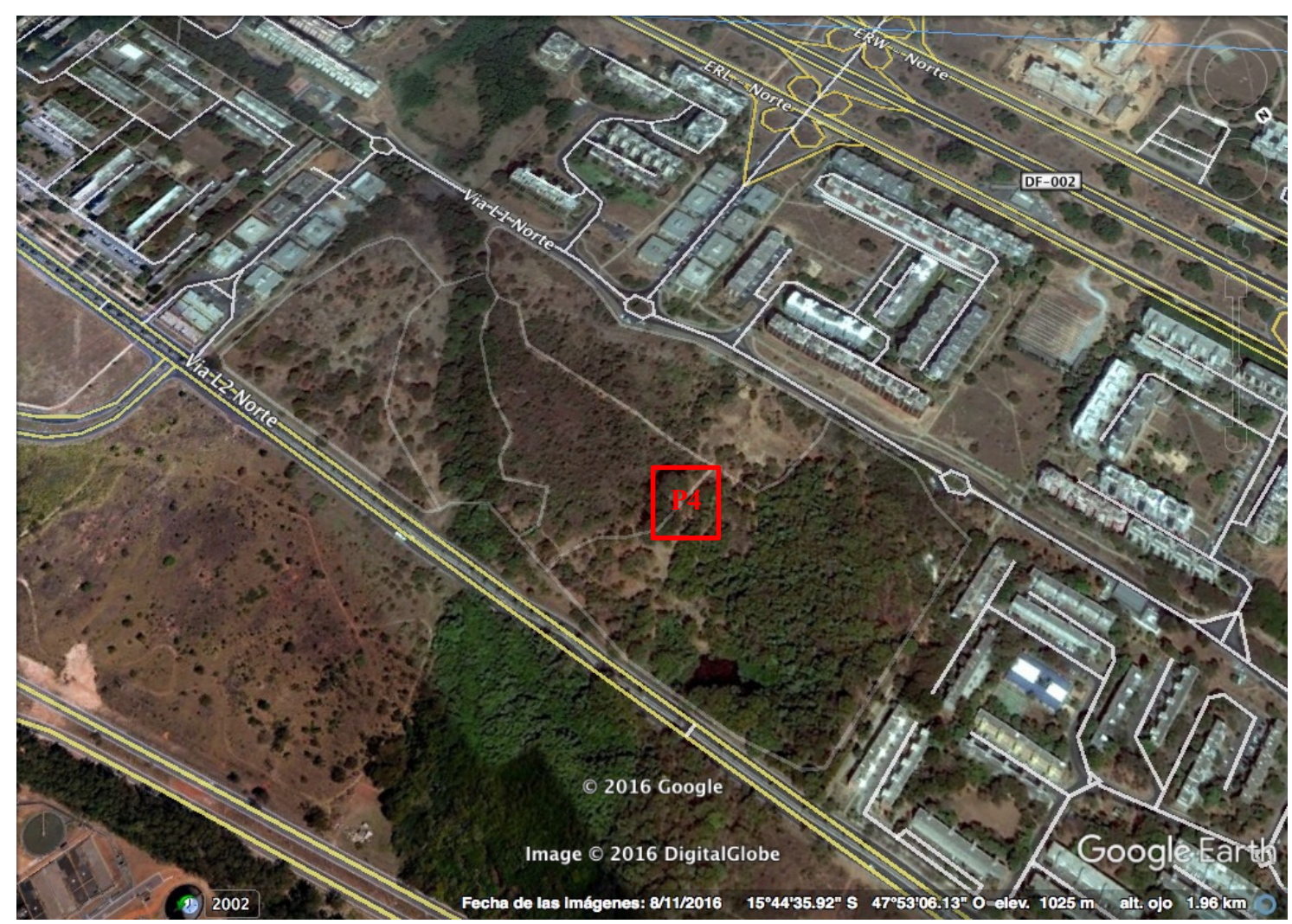

5. Centro de la ciudad de Brasilia (BRRO): El punto en el que se cruzan la avenida Eixo Rodoviario y Eixo Monumental es la estación central rodoviária metropolitana. Es un punto importante dado que es el punto de llegada y partida de buses que comunican a la ciudad con sus ciudades satélites (ciudades cercanas que hacen parte del distrito federal). Conocido como el corazón de Brasilia, la rodoviária metropolitana ofrece a los habitantes de la ciudad pequeñas tiendas de comercio, por lo que cercano al sector se tienen altos flujos de personas. En este punto se tienen los sectores comerciales norte y sur y el teatro nacional de Brasilia. La grabación binaural fue realizada a la salida sur-oriental de la rodoviária, paralela al Eixo Rodoviario sentido norte-sur (P5). 


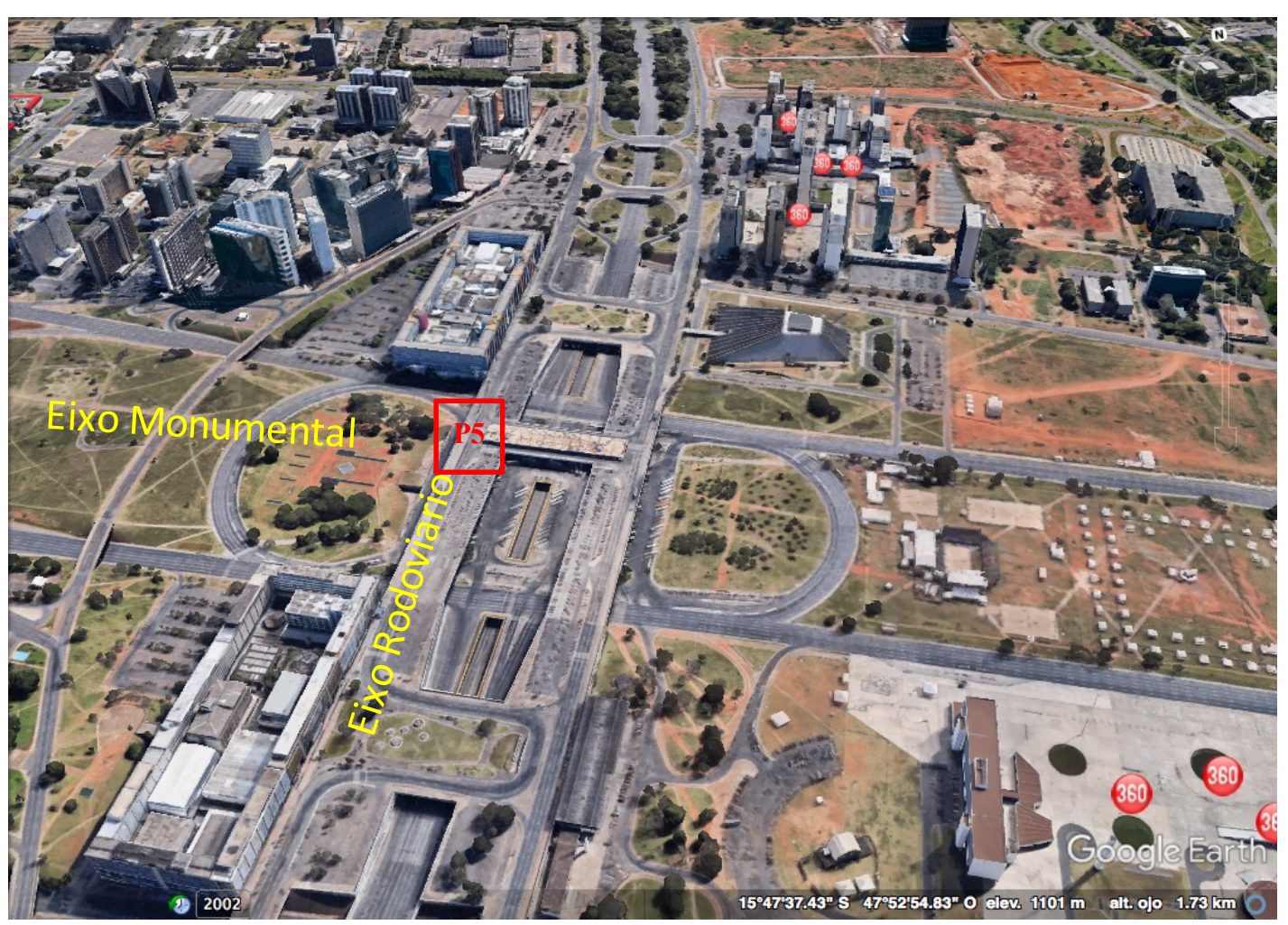

6. Autopista norte estación Alcalá (BOAPA): Intervenido por el distrito de Bogotá a principios del año 2000, el Parque Alcalá es un parque zonal de poco más de cuatro hectáreas, donde su tradicional bosque de eucaliptos acompaña una bien dotada zona de recreación y amplios espacios para el esparcimiento y descanso general. Ubicado en la localidad de Usaquén, entre las calles 136 y 138 y las avenidas 19 y carrera 13, el parque Nueva Autopista está rodeado de casas de 1 y 2 pisos, así como edificios de vivienda de máximo 6 pisos. Dentro de las actividades que se realizan en este espacio se cuenta con actividades físicas, descanso general, juegos infantiles, espacio de paseo con mascotas, danza y actividades culturales. Las principales vías vehiculares de este sector son la Autopista Norte y la Avenida 19. La autopista norte de Bogotá es una de las principales avenidas de la ciudad. En el punto de observación (sentido sur-norte a la altura de la estación Alcalá), la autopista está conformada por 4 vías, dos en sentido sur-norte y dos más en sentido norte-sur. En el sentido sur-norte una de las vías cuenta con 3 carriles para carros particulares y un carril más de uso exclusivo del sistema de transporte masivo Transmilenio, mientras que la segunda vía (denominada paralela) cuenta con dos carriles 
más. Para el sentido sur-norte en horas pico (entre 7 y 8 am) es posible alcanzar un volumen de unos 8000 vehículos por hora mientras que para el sentido norte-sur a la misma hora se puede tener un volumen de unos 4600 vehículos aproximadamente. La grabación binaural fue realizada a 25 metros de autopista en sentido sur-norte (P6).

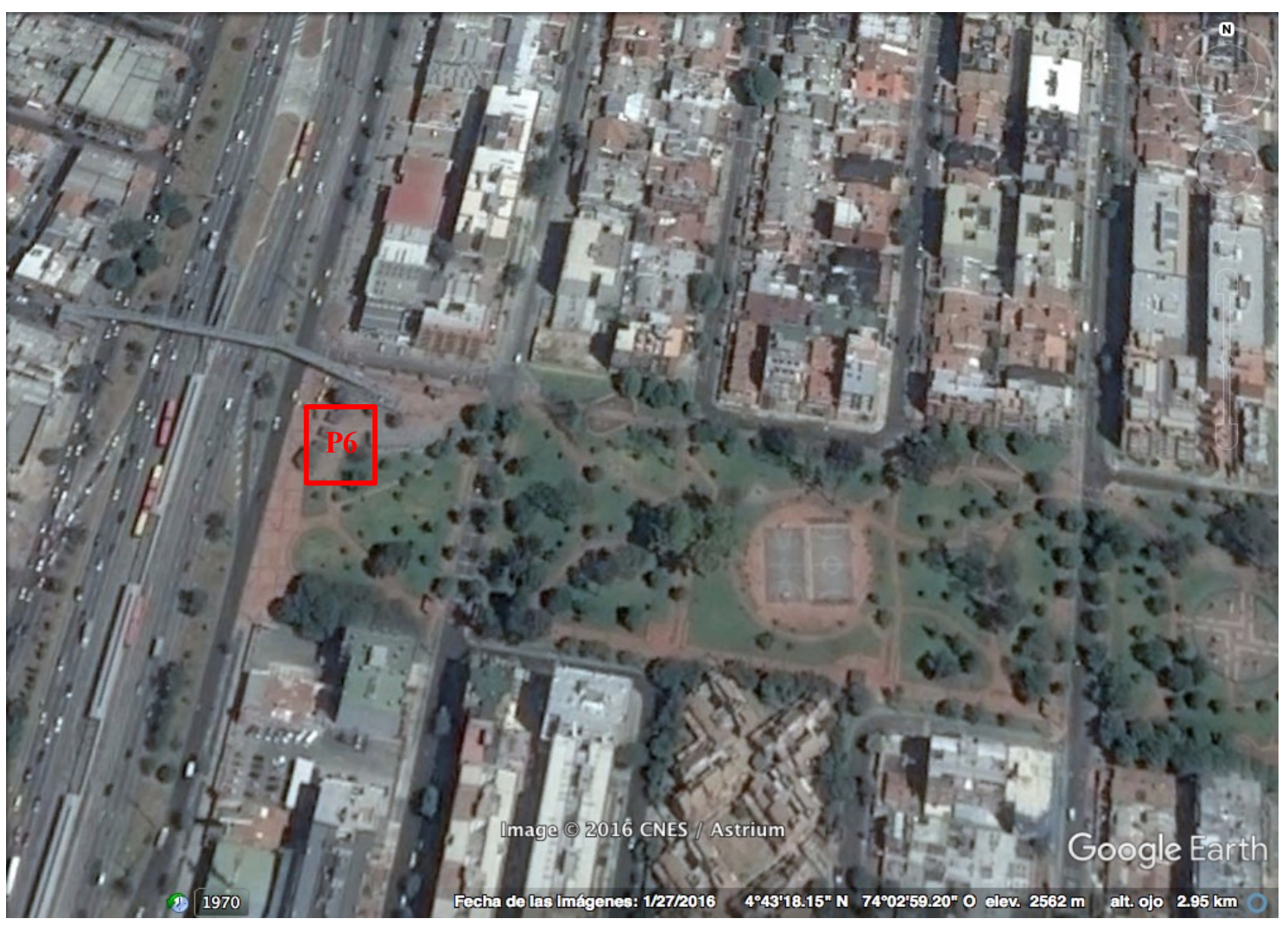

7. Calle 19 con Cra 7 (BOC197): Este es el centro de la ciudad de Bogotá. La calle 19 comunica el oriente con el occidente de la ciudad, mientras que la Cra 7 es uno de los emblemas de la ciudad y la cruza de norte a sur. La Cra séptima a la altura de la Cll 19 es de uso exclusivo para peatones, sin embargo, la Cll 19 si presenta un flujo vehicular, principalmente de buses y transporte público. Las edificaciones cercanas al punto de escucha alcanzan una altura de hasta 20 pisos, siendo un lugar concurrido por las personas, por lo que también es común encontrar vendedores ambulantes y comercio en las esquinas de este cruce de vías. La grabación binaural fue realizada paralela a la calle 19 sentido occidente-oriente (P7). 


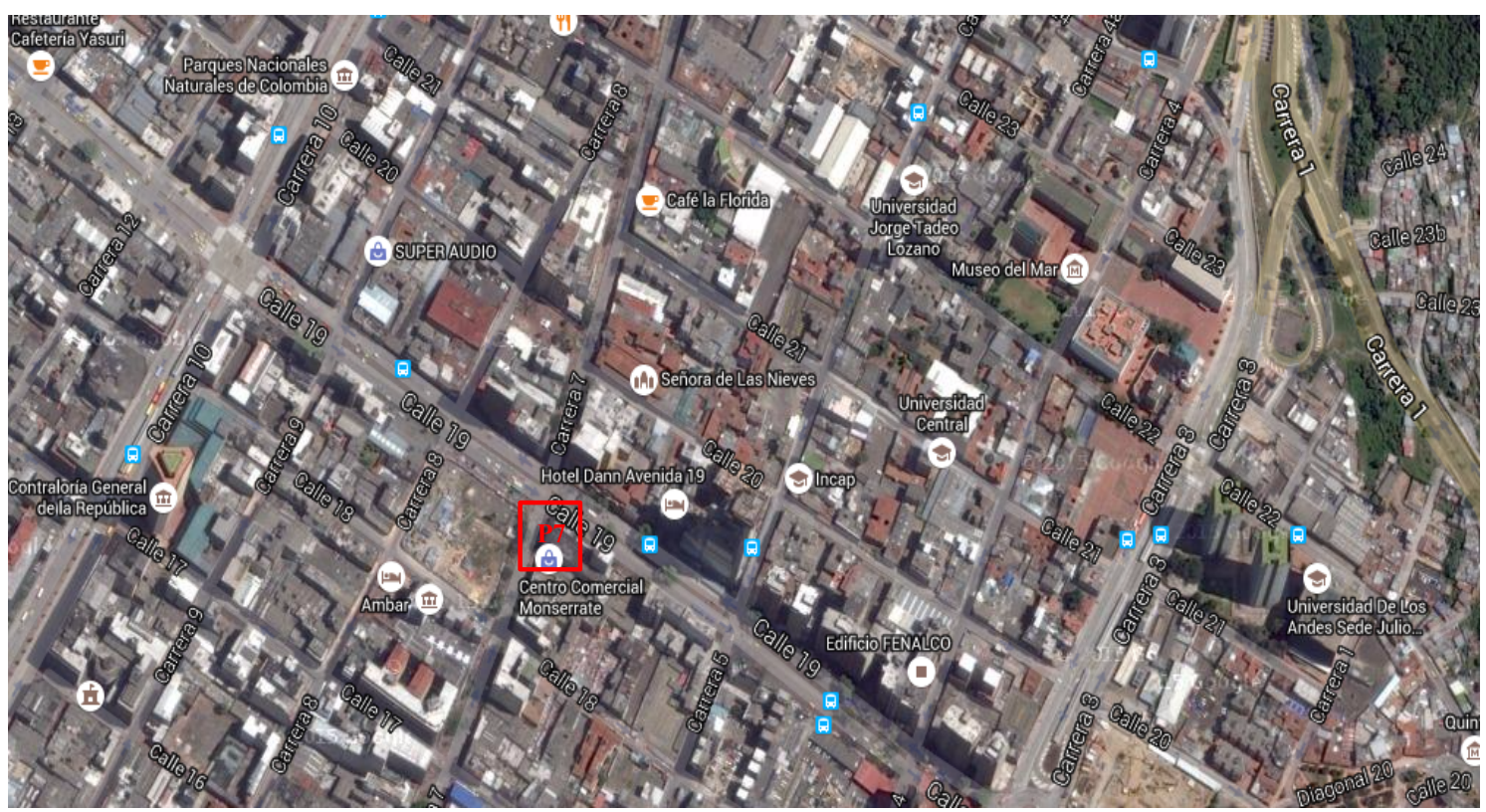

8. Fuente de agua edificio Tequendama (FAET): Esta fuente de agua está ubicada una plazoleta del edificio Tequendama, en un lugar tradicional del centro de Bogotá, cercana a la Plaza de toros, el observatorio planetario y el centro empresarial Tequendama. La plaza donde se encuentra la fuente queda ubicada sobre la carrera 7 , que a la altura de la calle 28 ya no es de uso exclusivo de peatones y presenta un flujo vehicular en horas pico cercano a los 3000 vehículos. La grabación binaural fue realizada en paralelo a la carrera séptima, en sentido sur-norte (P8).

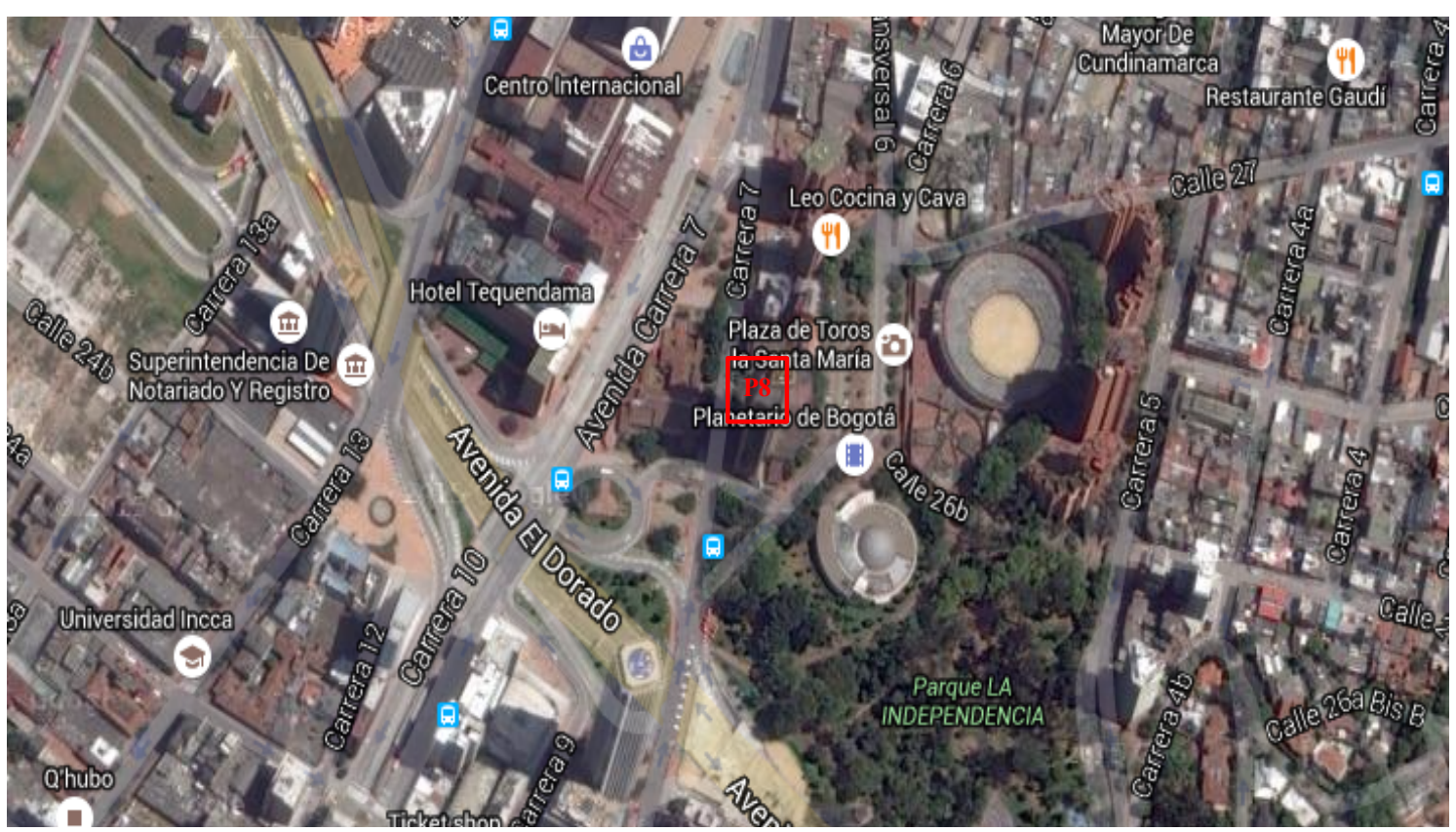


9. Plaza de Lourdes (PL): La plaza de Lourdes es otro lugar representativo de la ciudad de Bogotá. En él se encuentra la iglesia de nuestra Señora Lourdes, construida a finales del siglo XIX. El parque queda ubicado junto a la calle 63 Carrera 13, en la localidad de Chapinero de la ciudad de Bogotá. El tráfico vehicular es denso, de baja velocidad. En la plazoleta del parque se encuentran transeúntes, estudiantes, lustrabotas, vendedores ambulantes y es escenario tradicional de arte callejero. La grabación binaural fue realizada en el centro de la plaza, paralelo a la calle 63 sentido occidente-oriente (P9).

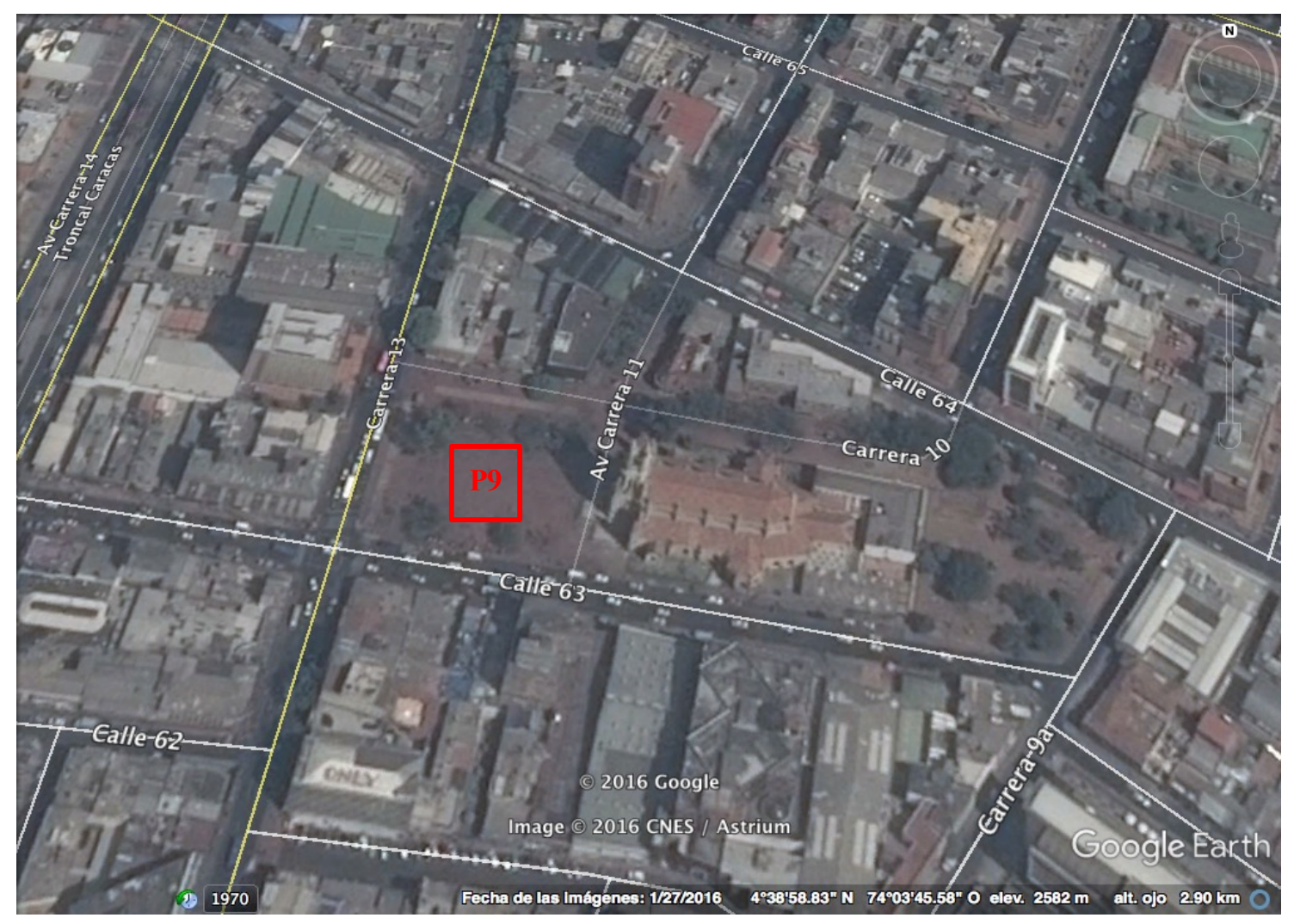

10. Parque central Simón Bolívar (PSB): Hace parte del gran complejo del parque Simón Bolívar (conformado por El parque de los Novios (parque el Lago), Parque Salitre, Complejo acuático, Centro de Alto rendimiento, la Biblioteca Virgilio Barco entre otros). La historia del parque Central se remonta al año 1966, aunque en el año 1968, con la visita del Papa Juan Pablo II se construyó un pequeño templete a fin de la celebración de una 
misa campal. A partir de ahí, el parque ha tenido intervenciones en los años 1979 (ocasión de los 200 años del Libertador Simón Bolívar), aunque en 1983 quedó constituido oficialmente como parque. En 1995 la administración distrital decidió recuperar el lago principal y ofrecer al público un espacio con ciclo-rutas y dotación general para prácticas deportivas. Este parque es de escala metropolitana y en la actualidad cuenta con zonas de juegos, plaza de eventos, monumentos, 16 kilómetros de red de caminos y 11 kioskos. Las actividades desarrolladas son eventos musicales y festivales, descanso, prácticas deportivas y en general diversas actividades recreativas. Se encuentra ubicado entre las Calles 53 y 63 y las carreras 68 y la avenida la esmeralda. La avenida 68 también presenta gran flujo vehicular, pues comunica el occidente con el norte de la ciudad (P10).

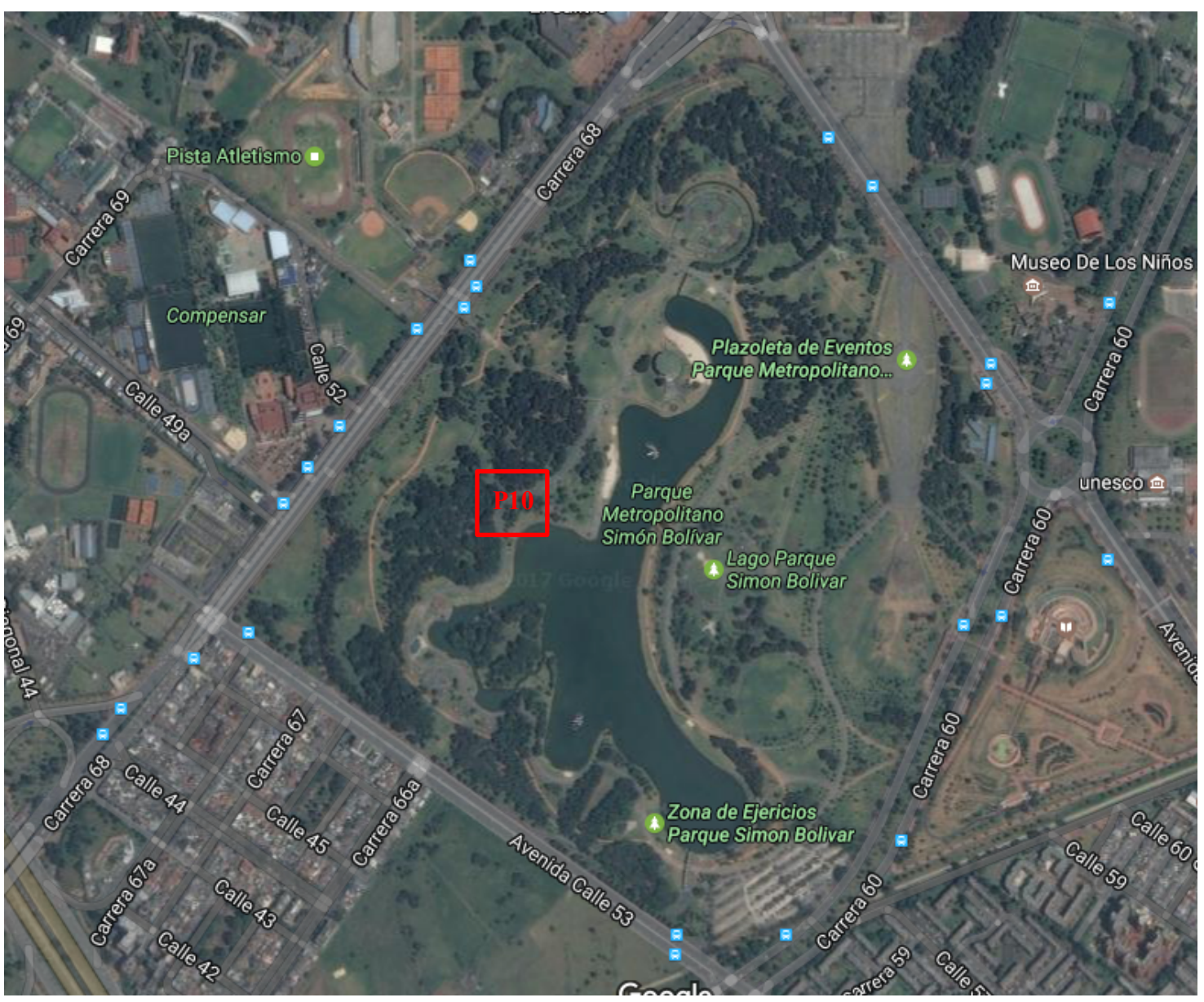




\section{Anexo 2. Especificaciones técnicas del sistema de grabación binaural (datos según fabricantes)}

Micrófono Binaural Roland 10CS-EM (sin insertar al oído)

Element: Electret Condenser

Polar Pattern: Omnidirectional

Frequency Response: $20 \mathrm{~Hz}$ to $20,000 \mathrm{~Hz}$

Open Circuit Sensitivity: $-40 \mathrm{~dB} 1 \mathrm{~V} / \mathrm{Pa}$

S/N: Greater than $60 \mathrm{~dB}$

Impedance: $2.2 \mathrm{k} \Omega$

Current Consumption: $500 \hat{\mathrm{I}}^{1} / 4 \mathrm{~A}$

Power Requirements $2 \mathrm{~V}$ to $10 \mathrm{~V}$

Grabadora Zoom H4N

ASPECTOS GENERALES

Number of

simultaneous recording

tracks:

2 (STEREO mode), 4 (4CH mode), 2 (MTR mode)

Number of

2 (STEREO mode), 4 (4CH mode), 4 (MTR mode)

simultaneous playback

tracks:

Recording time 4GB Approx. 380 minutes (WAV $44.1 \mathrm{kHz} / 16$ bit stereo (SDHC): track)

Approx. 68 hours (MP3 $44.1 \mathrm{kHz} / 128 \mathrm{kbps}$ stereo track)

* Recording times are approximations. Actual times may differ according to recording conditions.

Maximum number of $2 \mathrm{~GB}$

simultaneous recording

files size:

Projects:

1000/card 


\section{ASPECTOS GENERALES}

\begin{tabular}{|c|c|}
\hline Locate function: & Hours/Minutes/Seconds/Milliseconds \\
\hline Other functions: & Punch-in/out, Bounce, A-B repeat \\
\hline \multirow[t]{4}{*}{ Effects modules: } & 2 \\
\hline & STEREO / 4CH Mode: LO CUT, \\
\hline & COMP/LIMITER \\
\hline & MTR Mode : PRE AMP module, EFX module \\
\hline Types: & 50 \\
\hline Patches: & 60 \\
\hline \multirow[t]{2}{*}{ Tuners: } & Chromatic, Guitar, Bass, Open \\
\hline & $\mathrm{A} / \mathrm{D} / \mathrm{E} / \mathrm{G}, \mathrm{DADGAD}$ \\
\hline sources: & 5 \\
\hline Variable beat: & $1 / 4-8 / 4,6 / 8$, unaccentuated \\
\hline Tempo: & $40.0-250.0 \mathrm{BPM}$ \\
\hline A/D conversion: & 24 bit $\times 128$ oversampling \\
\hline D/A conversion: & 24 bit $\times 128$ oversampling \\
\hline Recording media: & SD card $(16 \mathrm{MB}-2 \mathrm{~GB}), \mathrm{SDHC}$ card $(4 \mathrm{~GB}-32 \mathrm{~GB})$ \\
\hline Display: & $128 \times 64$ dots Full-dot backlit LCD \\
\hline \multicolumn{2}{|c|}{ DATA TYPE - WAV FORMAT (RECORD/PLAY) } \\
\hline Quantization: & $16 / 24 \mathrm{bit}$ \\
\hline Sampling frequency: & $44.1 / 48 / 96 \mathrm{kHz}$ \\
\hline \multicolumn{2}{|c|}{ DATA TYPE - MP3 FORMAT (RECORDING) } \\
\hline
\end{tabular}




\section{ASPECTOS GENERALES}

Bit rate:

$48,56,64,80,96,112,128,160,192,224,256,320$

kbps, VBR

Sampling frequency: $\quad 44.1 \mathrm{kHz}$ 


\section{Anexo 3. Características y calibración del sistema de reproducción}

El sistema de reproducción usado en las pruebas subjetivas está conformado por Auriculares Beyerdynamic DT 770, placa de sonido RME Babyface pro y torso artificial $01 \mathrm{~dB}$.

\section{A3.1 Especificaciones técnicas Beyerdynamic DT 770}

Transducer type Dynamic

Operating principle Closed

Frequency response. $5-35,000 \mathrm{~Hz}$

Nominal impedance $32 \Omega / 80 \Omega / 250 \Omega$

Nominal SPL $96 \mathrm{~dB}$

T.H.D $<0.2 \%$

Power handling capacity $100 \mathrm{~mW}$

Sound coupling to the ear Circumaural Ambient noise attenuation $32 \Omega$ version approx.20dBA

$80 \Omega / 250 \Omega$ version approx.18dBA

Nominal headband pressure approx. $3.5 \mathrm{~N}$

Weight(withoutcable) $.270 \mathrm{~g}$

Por medio de los softwares Smaart live y SysTune y el torso artificial 01dB fueron aplicadas pruebas de coherencia entre señales generadas y la salida de los Auriculares, obteniendo valores de coherencia del $99 \%$ entre los $40 \mathrm{~Hz}$ y los $16000 \mathrm{~Hz}$. La respuesta en frecuencia del sistema no presentó variaciones de $\pm 6 \mathrm{~dB}$ entre $20 \mathrm{~Hz}$ y $20000 \mathrm{~Hz}$.

\section{A3.2 Características Torso artificial 01dB}

El torso artificia 01dB está compuesto por un preamplificador GRAS 12AA y micrófonos MK231 SET. La respuesta en frecuencia de dichos micrófonos se presenta a continuación. 


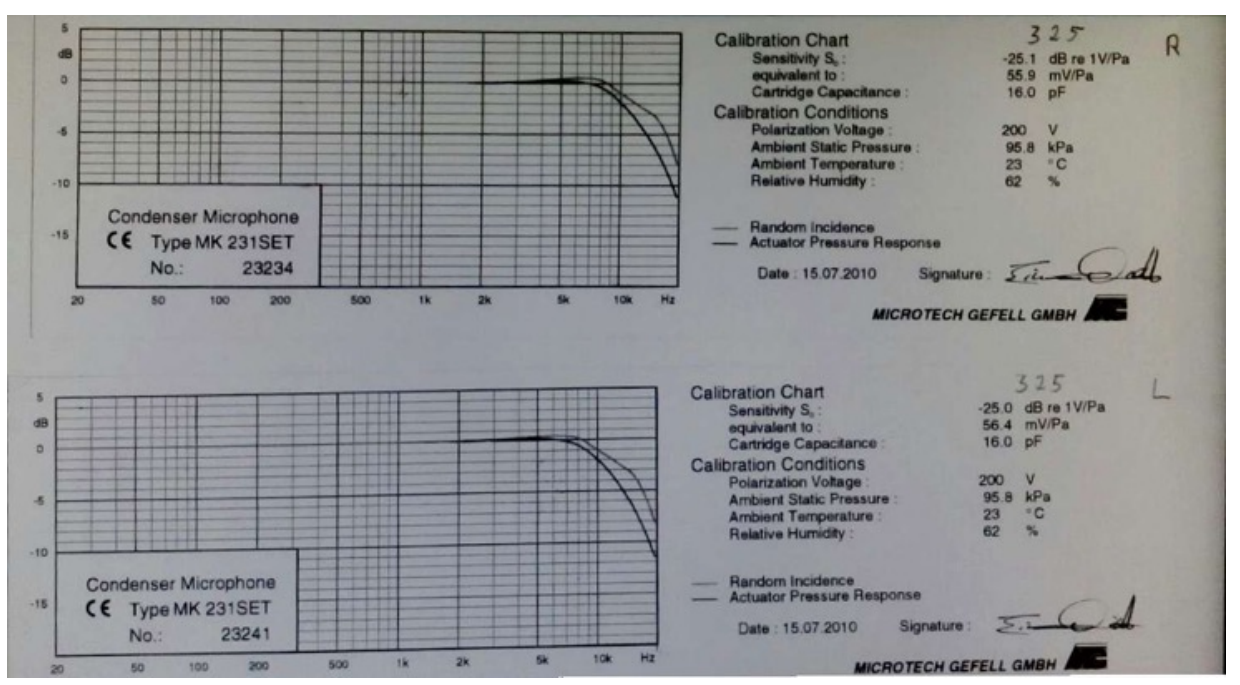

\section{A3.3 Proceso de calibración sistema de reproducción}

El proceso de calibración del sistema de reproducción está dividido en dos partes. El primero consistió en grabar una señal de referencia en el sistema de reproducción (micrófonos binaurales y grabadora). La señal de referencia usada fue generada por un calibrador Cesva Tipo I, generando 94 dB a 1000Hz. Esta señal se grabó a dos niveles de ganancia digital: -40dBFS y -50dBFS.

La segunda parte de la calibración consistió en fijar una señal de referencia al torso artificial (nuevamente se usó el calibrador Cesva tipo I generando 94 dB a $1000 \mathrm{~Hz}$ ) y posterior a esto, usando los auriculares DT 770, se reprodujeron las señalas de referencias grabadas en el sistema de grabación y se ajustaron los niveles de salida hasta obtener a la salida del torso artificial los mismos $94 \mathrm{~dB}$. El registro fotográfico de dicho proceso se presenta a continuación:
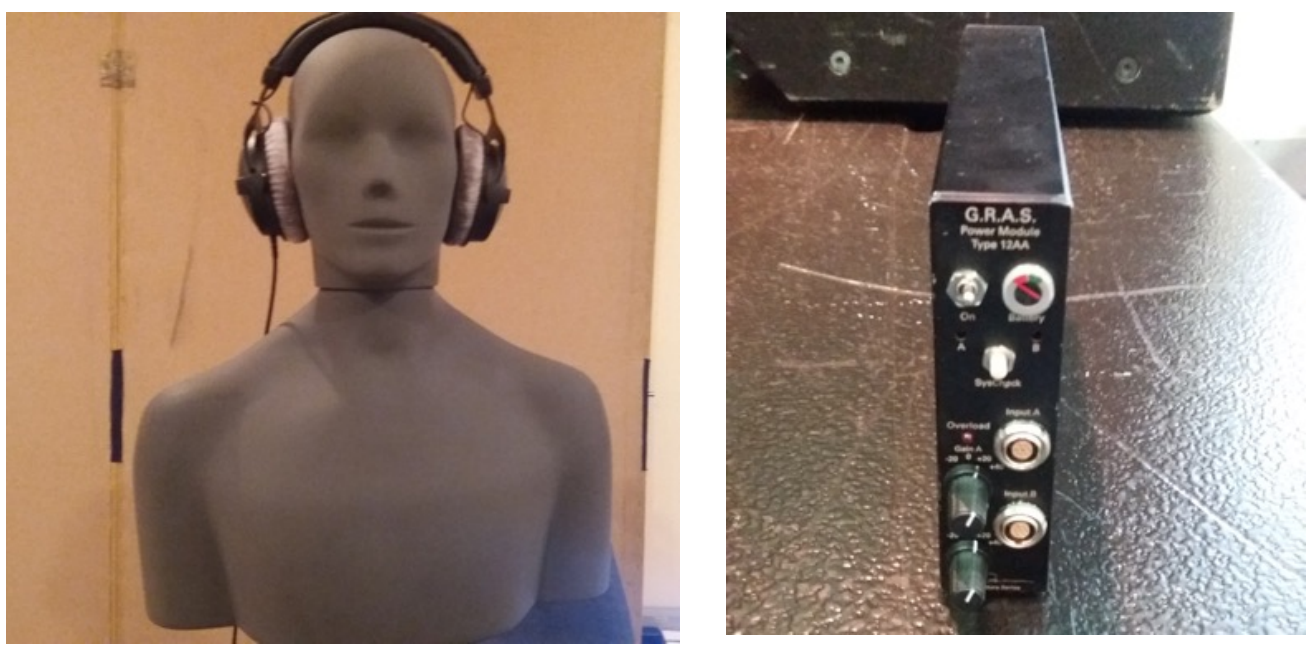
a. Torso artificial $01 \mathrm{~dB}$ y

b. Preamplificador GRAS 12AA auriculares DT 770

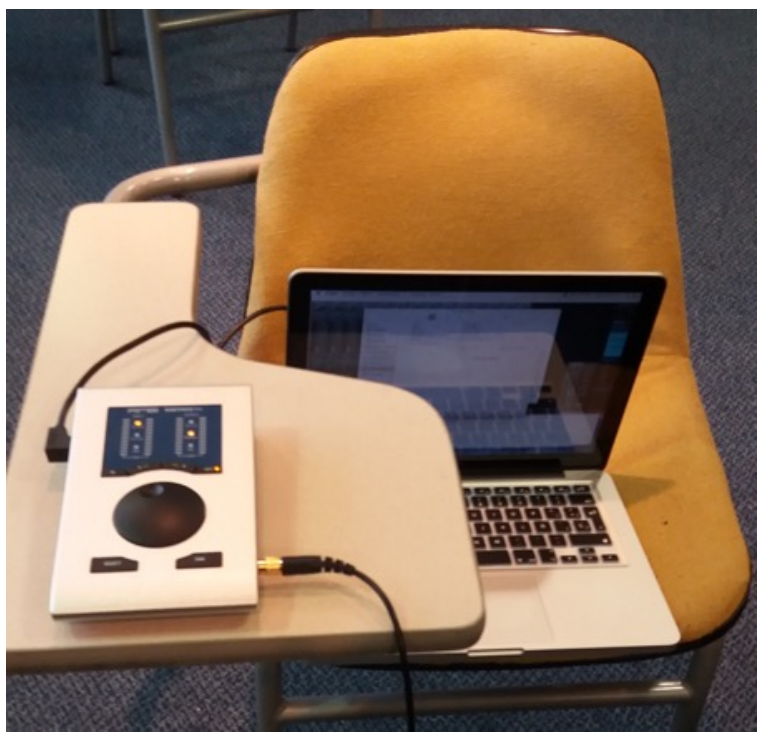

c. Sistema de reproducción: Placa de sonido Babyface PRO

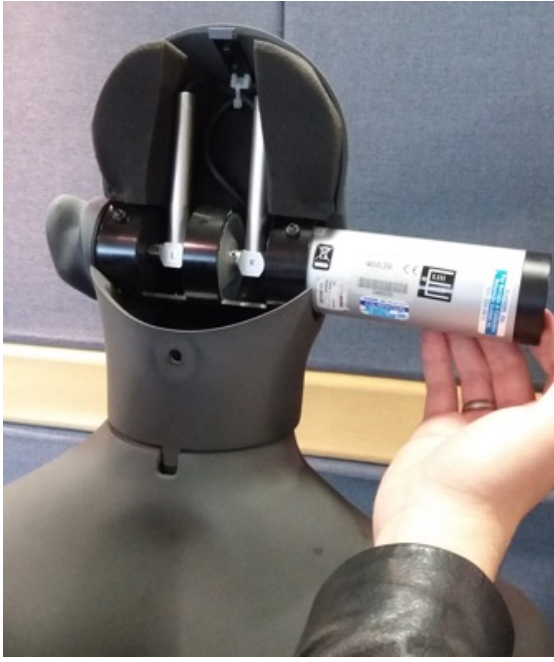

d. Calibración micrófono izquierdo

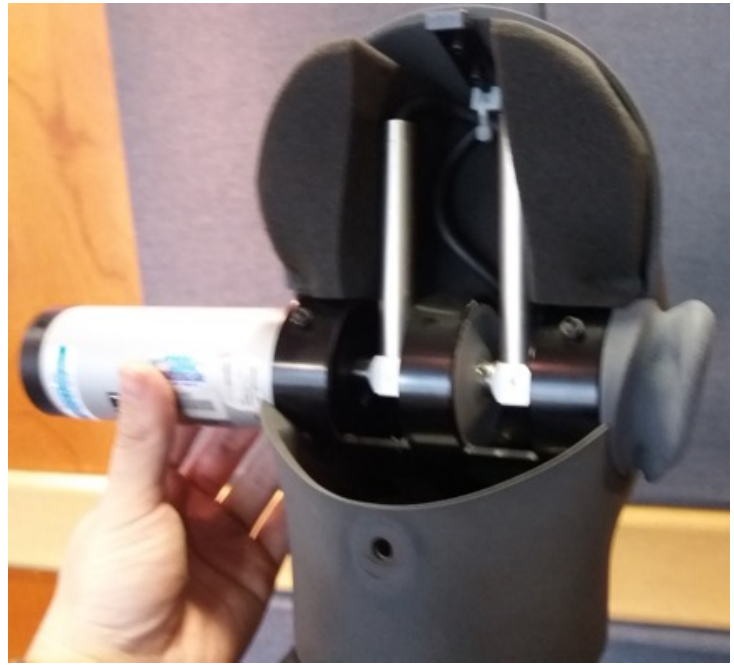

e. Calibración micrófono derecho

Proceso de calibración de torso artificial 01 dB con calibrador Cesva tipo I 


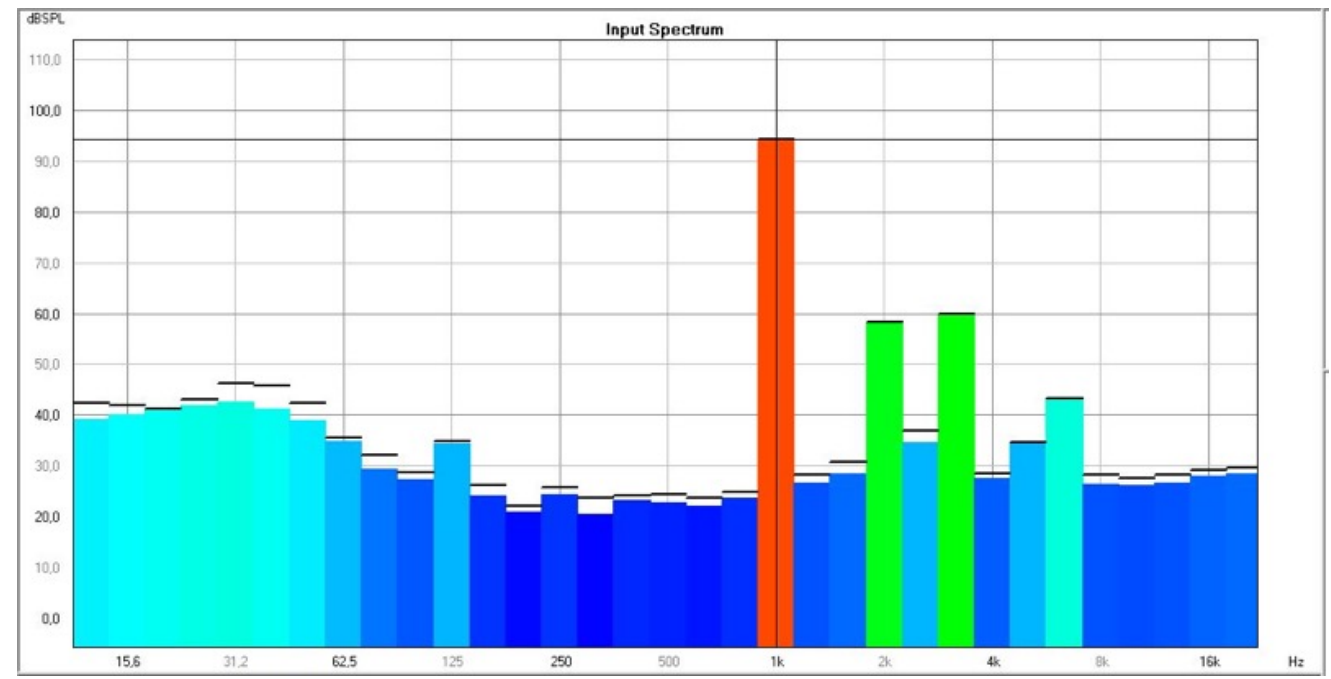

f. Ajuste de sistema de reproducción. Calibración de señal de referencia a -40dB izquierda.

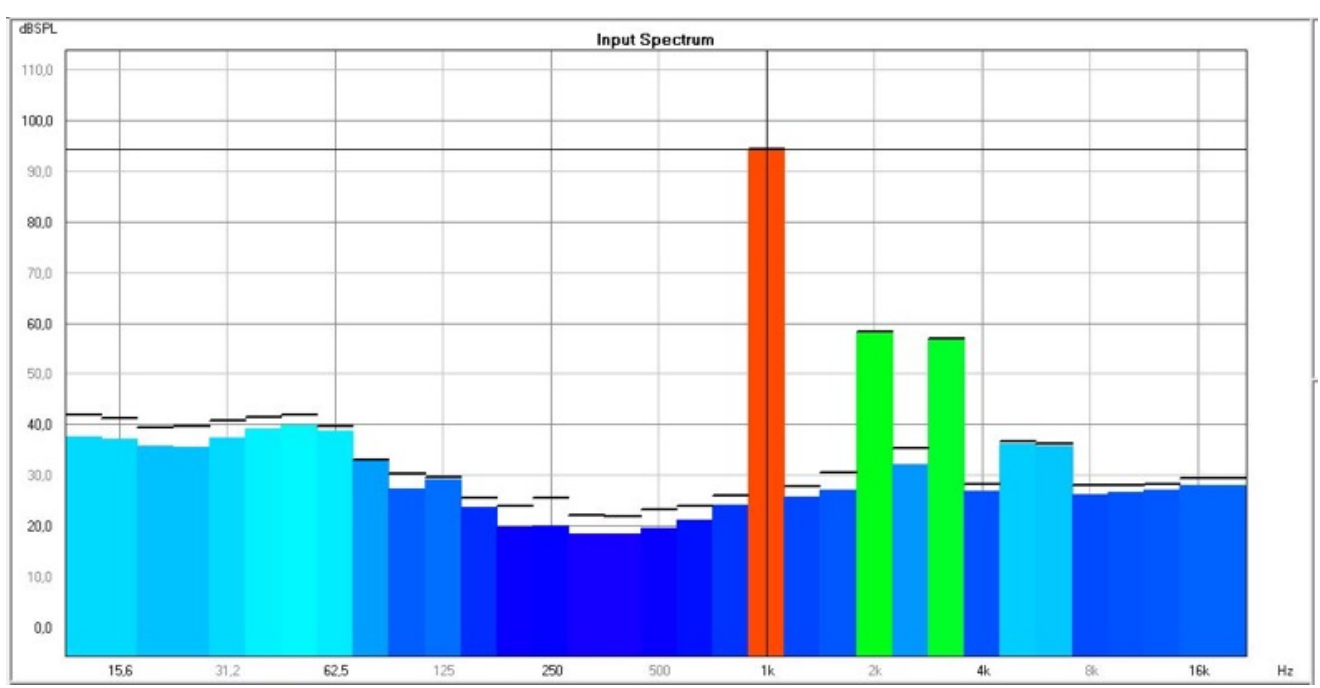

g. Ajuste de sistema de reproducción. Calibración de señal de referencia a -40dB derecha 


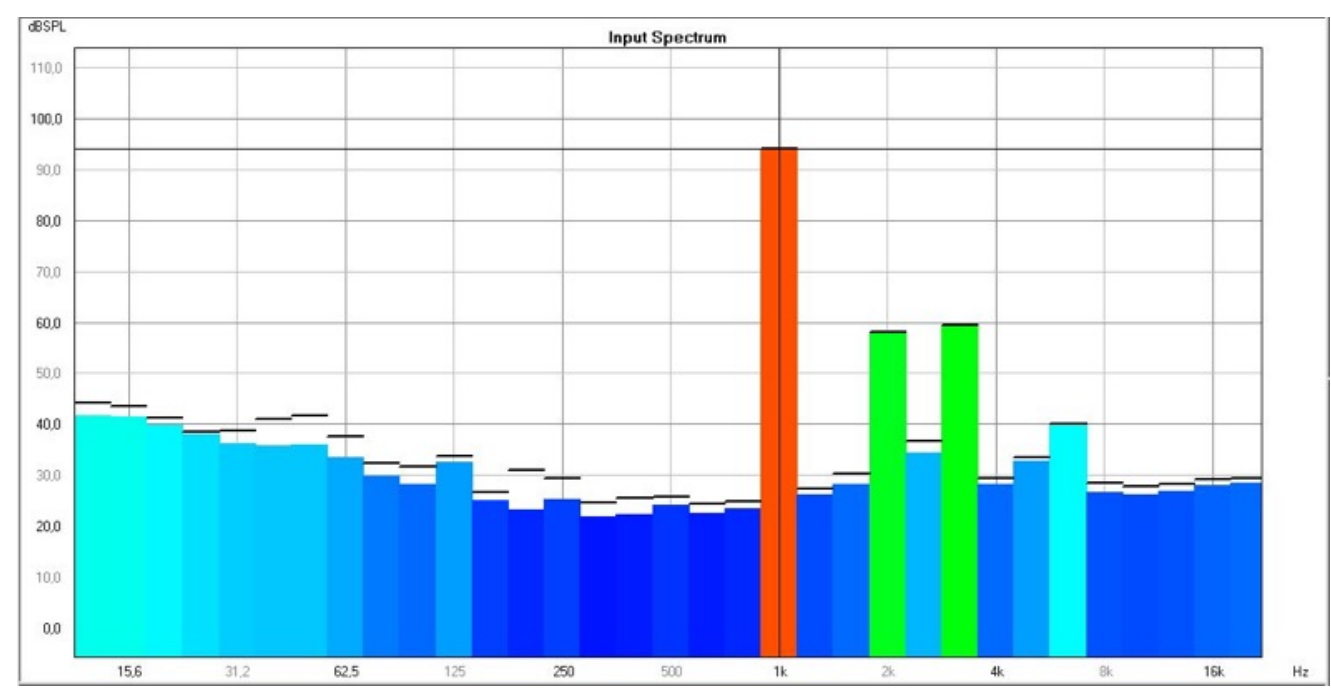

h. Ajuste de sistema de reproducción. Calibración de señal de referencia a -50dB izquierda

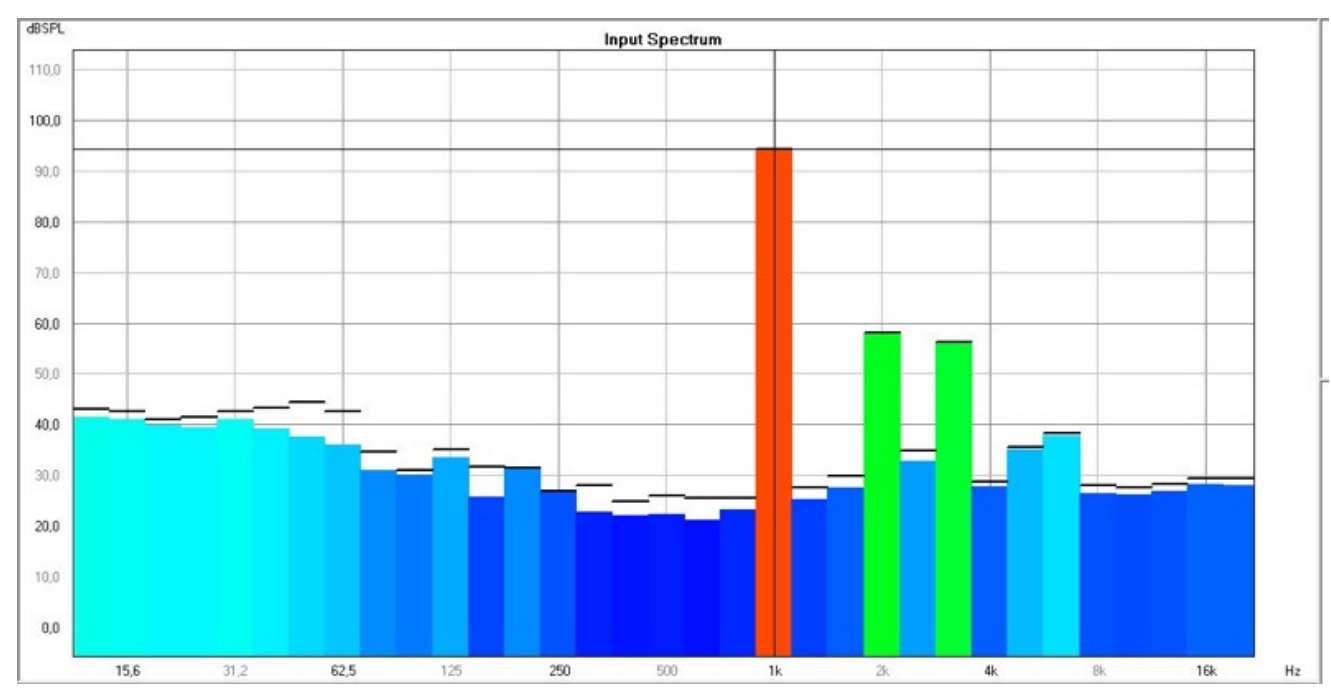

i. Ajuste de sistema de reproducción. Calibración de señal de referencia a -50dB derecha 


\section{Anexo 4. Herramienta de evaluación subjetiva prueba en laboratorio.}

\begin{tabular}{|c|c|c|c|c|c|c|}
\hline \multicolumn{2}{|c|}{ Lugar de grabación: $\mathrm{XXXX}$} & & & & & \\
\hline \multicolumn{7}{|c|}{ Hasta que punto escucha las siguientes fuentes sonoras: } \\
\hline & & Nada & Poco & Algo & Mucho & $\begin{array}{l}\text { Completamen } \\
\text { te dominante }\end{array}$ \\
\hline \multicolumn{2}{|c|}{$\begin{array}{l}\text { 1. Tráfico (carros, buses, } \\
\text { motocicletas, aviones, etc.) }\end{array}$} & $\square$ & $\square$ & $\square$ & $\square$ & $\square$ \\
\hline \multicolumn{2}{|c|}{$\begin{array}{l}\text { 2. Sonidos de motores (sistemas } \\
\text { de ventilación, etc.) }\end{array}$} & $\square$ & $\square$ & $\square$ & $\square$ & $\square$ \\
\hline \multicolumn{2}{|c|}{$\begin{array}{l}\text { 3. Otros sonidos (industria, } \\
\text { construcción, comercio, sirenas, }\end{array}$} & $\square$ & $\square$ & $\square$ & $\square$ & $\square$ \\
\hline \multicolumn{2}{|c|}{$\begin{array}{l}\text { 4. Sonidos humanos (voces, } \\
\text { pasos, niños jugando, risas, etc.) }\end{array}$} & $\square$ & $\square$ & $\square$ & $\square$ & $\square$ \\
\hline \multicolumn{2}{|c|}{$\begin{array}{l}\text { 5. Sonidos naturales (pájaros, } \\
\text { agua, viento, etc.) }\end{array}$} & $\square$ & $\square$ & $\square$ & $\square$ & $\square$ \\
\hline \multicolumn{6}{|c|}{ ¿Cual de estos sonidos considera que caracteriza o identifica esta grabación? } & \\
\hline $\begin{array}{l}\text { máquinas y } \\
\text { equipamiento }\end{array}$ & Tráfico & $\begin{array}{l}\text { Sonidos } \\
\text { naturales }\end{array}$ & $\begin{array}{l}\text { Sonidos } \\
\text { humanos }\end{array}$ & $\begin{array}{l}\text { Otros } \\
\text { (cuales?) }\end{array}$ & & \\
\hline$\square$ & $\square$ & $\square$ & $\square$ & $\square$ & & \\
\hline
\end{tabular}

\begin{tabular}{|l|l|l|l|l|l|l|}
\hline \multicolumn{6}{|l|}{ De forma general: ¿cómo evalúa el ambiente sonoro presentado en la grabación? } \\
\hline Muy bueno & Bueno & Neutro & Malo & Muy Malo & & \\
\hline$\square$ & $\square$ & $\square$ & $\square$ & $\square$ & \\
\hline
\end{tabular}

¿En que extensión considera que los siguientes 8 factores describen el ambiente sonoro escuchado? Indique una opción para cada factor:

\begin{tabular}{|c|c|c|c|c|c|c|}
\hline \multicolumn{2}{|c|}{ El ambiente sonoro es: } & $\begin{array}{l}\text { Concuerdo } \\
\text { completamen } \\
\text { te }\end{array}$ & $\begin{array}{l}\text { Concuerdo } \\
\text { bastante }\end{array}$ & $\begin{array}{l}\text { acuerdo ni } \\
\text { en } \\
\text { desacuerdo }\end{array}$ & $\begin{array}{l}\text { Parcialment } \\
\text { e en } \\
\text { desacuerdo }\end{array}$ & $\begin{array}{l}\text { Totalmente } \\
\text { en } \\
\text { desacuerdo }\end{array}$ \\
\hline \multicolumn{2}{|c|}{ Agradable } & $\square$ & $\square$ & $\square$ & $\square$ & $\square$ \\
\hline \multicolumn{2}{|l|}{ Caótico } & $\square$ & $\square$ & $\square$ & $\square$ & $\square$ \\
\hline \multicolumn{2}{|c|}{ Excitante/vibrante } & $\square$ & $\square$ & $\square$ & $\square$ & $\square$ \\
\hline \multicolumn{2}{|c|}{ Uniforme (Sin eventos) } & $\square$ & $\square$ & $\square$ & $\square$ & $\square$ \\
\hline \multicolumn{2}{|l|}{ Calmo } & $\square$ & $\square$ & $\square$ & $\square$ & $\square$ \\
\hline Desagradable & & $\square$ & $\square$ & $\square$ & $\square$ & $\square$ \\
\hline \multicolumn{2}{|l|}{ Agitado/variado } & $\square$ & $\square$ & $\square$ & $\square$ & $\square$ \\
\hline Monótono & & $\square$ & $\square$ & $\square$ & $\square$ & $\square$ \\
\hline \multicolumn{7}{|c|}{ ¿Conoce y/o ha visitado el lugar donde fue hecha esta grabación? } \\
\hline $\mathrm{Si}$ & $\square$ & No & $\square$ & & & \\
\hline \multicolumn{7}{|c|}{$\begin{array}{l}\text { ¿Cuanto tiempo permanecería en un lugar con un ambiente sonoro como el presentado en esta } \\
\text { grabación? }\end{array}$} \\
\hline $\begin{array}{l}\text { menos de } 10 \\
\text { minutos }\end{array}$ & $\begin{array}{l}\text { entre } 10 \text { y } 30 \\
\text { minutos }\end{array}$ & $\begin{array}{l}\text { entre } 30 \text { y } 60 \\
\text { minutos }\end{array}$ & $\begin{array}{l}\text { entre } 1 \text { y } 2 \\
\text { horas }\end{array}$ & $\begin{array}{l}\text { más de } 2 \\
\text { horas }\end{array}$ & & \\
\hline$\square$ & $\square$ & $\square$ & $\square$ & $\square$ & & \\
\hline \multicolumn{7}{|c|}{ ¿Que uso daría a un lugar con un ambiente sonoro como este? } \\
\hline Act. Física & Lugar de pasaje & $\begin{array}{l}\text { Contemplació } \\
\mathrm{n}\end{array}$ & $\begin{array}{l}\text { Lectura y } \\
\text { meditación }\end{array}$ & $\begin{array}{l}\text { Descanso } \\
\text { general }\end{array}$ & Trabajo & Otro (cual) \\
\hline$\square$ & $\square$ & $\square$ & $\square$ & $\square$ & $\square$ & $\square$ \\
\hline
\end{tabular}


Chapter Four. Final considerations, Conclusions and Future Work

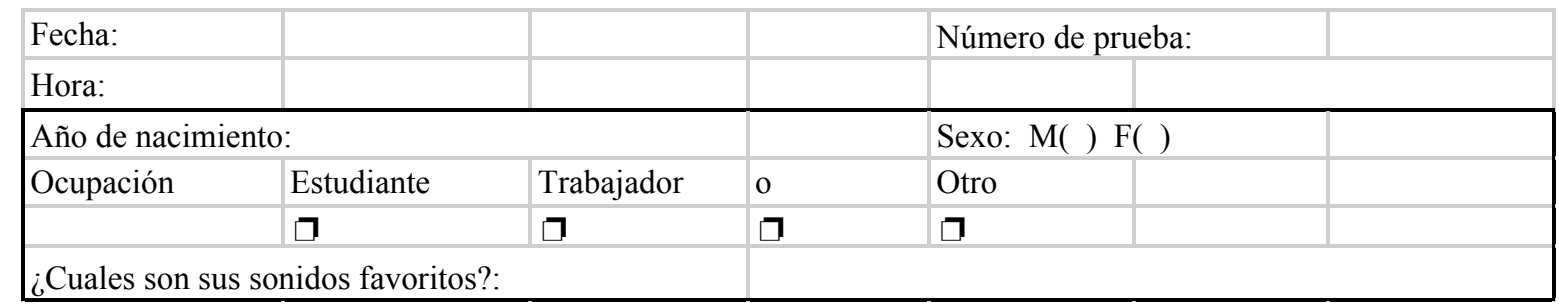




\section{Anexo 5. Lugares en estudio en Lisboa (Portugal).}

Jardim da fundaçao Gulbenkian: Fue construido en la década de los años 60 por los Arquitectos paisajistas António Biana Barreto y Gonçalo Riveiro. Actualmente es considerado uno de los jardines emblemáticos del modernismo portugués y en sus 7,5 hectáreas se cuenta con caminos aromáticos, recreación de vegetación típicamente portuguesa, aves, pequeños lagos, mesas y sillas para lectura y amplias zonas verdes. Se encuentra rodeado por las Avenidas Antonio Augusto de Aguiar y Berna, así como de la Rua Marqués Sa de Bandeira. Las edificaciones que le rodean tienen hasta 10 pisos. Las grabaciones binaurales fueron realizadas paralelo a la Avenida Berna (P11).

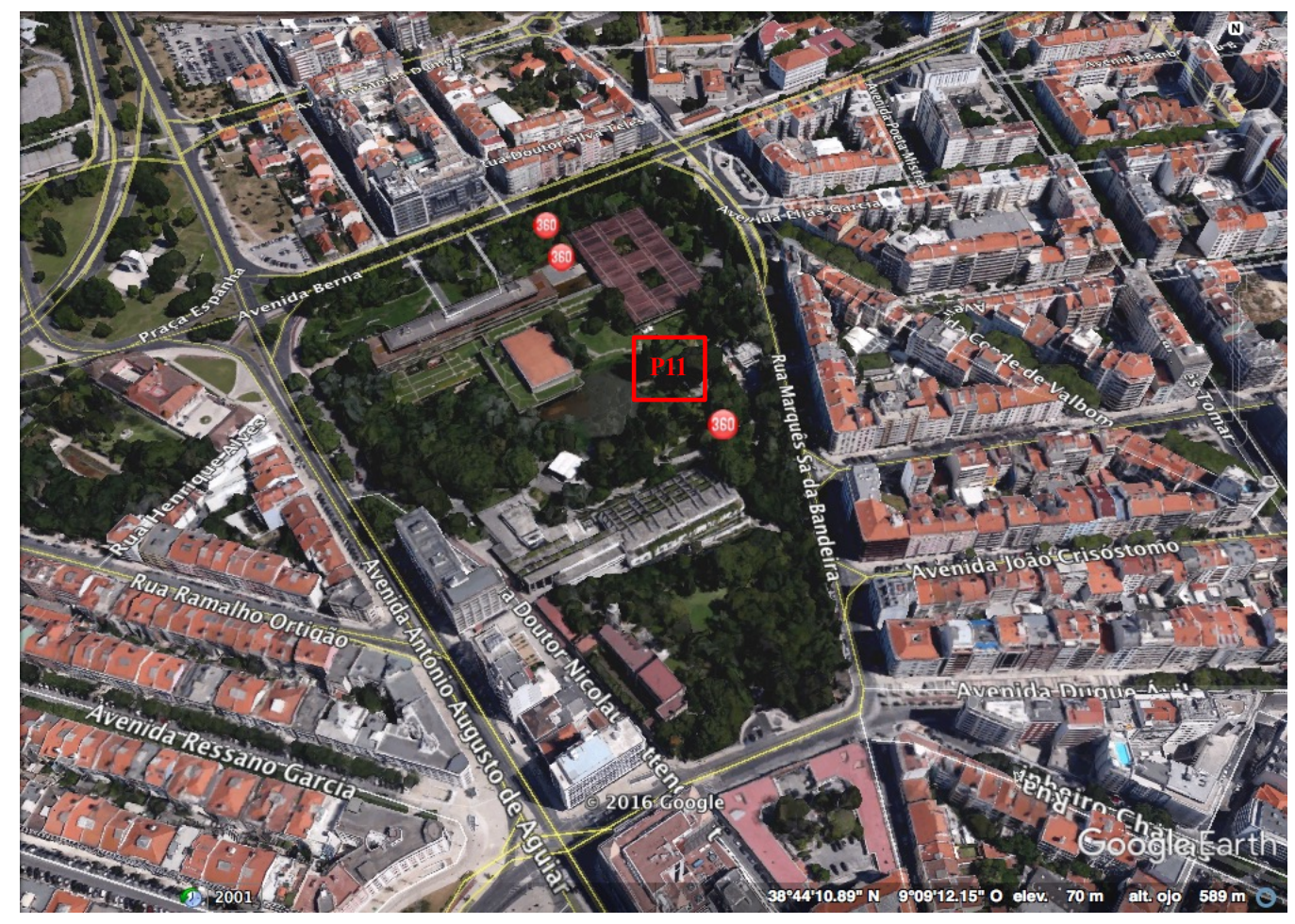

Jardim da Estrela: este parque ubicado en el barrio Lapa de la ciudad de Lisboa fue construido a finales del siglo XIX. En sus 4,6 hectáreas se cuenta con dos lagos, parques infantiles, kioscos y equipamiento para ejercitase físicamente. En los lagos pueden verse patos, cisnes y gansos, y rondando el jardín se encuentra un majestuoso pavo real. En el centro del jardín se cuenta con una glorieta en hierro que es usado generalmente para conciertos de bandas filarmónicas y disfrute general. Una de sus calles colinda con la Basílica da Estrela de Lisboa, punto obligado para turistas que además pueden llegar al lugar en el mítico tranvía de madera E25. En épocas de verano son comunes conciertos y picnics de 
lisboetas y turistas que llegan a disfrutar de este espacio. Las grabaciones fueron realizadas paralelo a la Rua Estrela (P12).

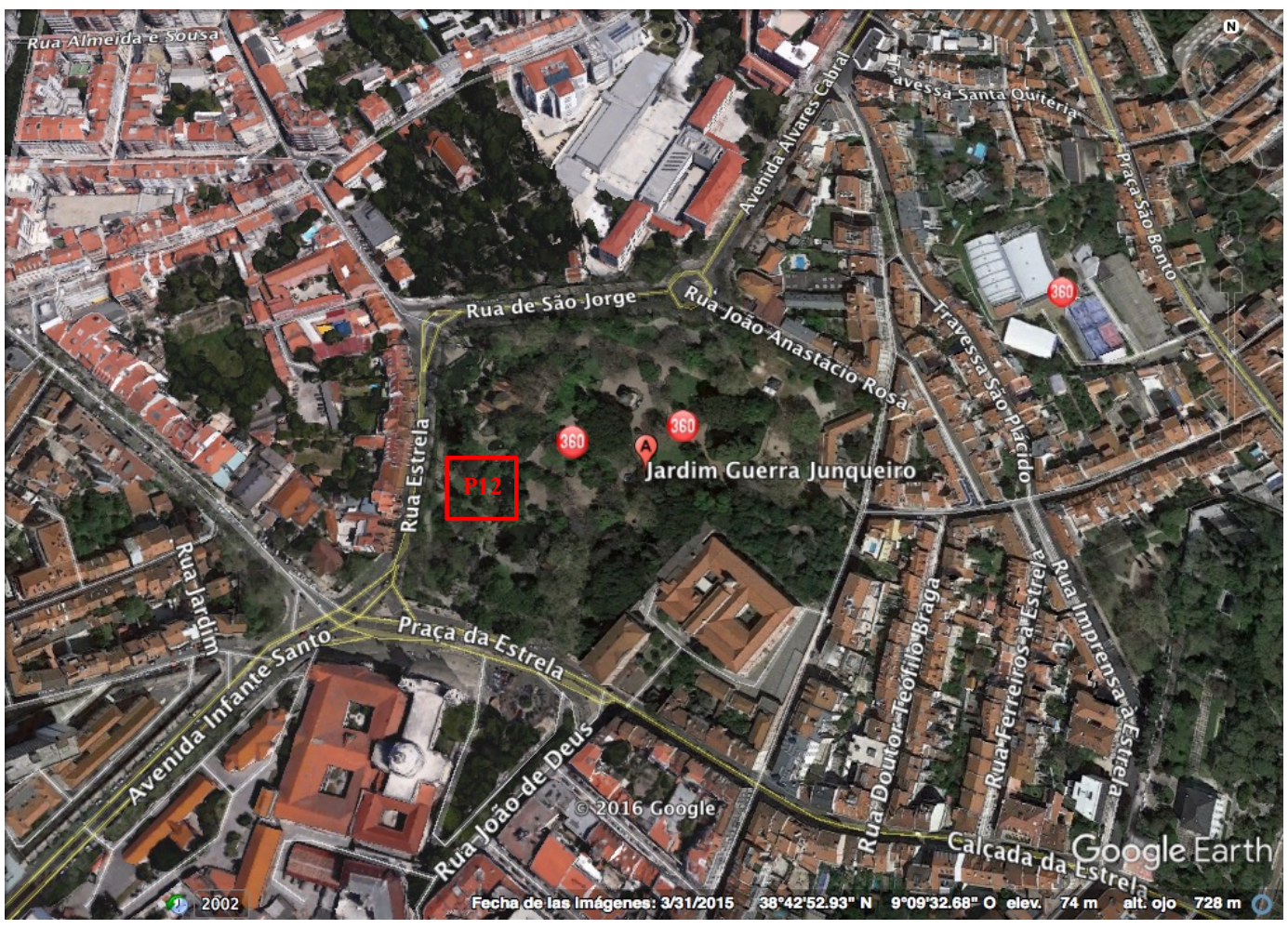

Jardim do Príncipe Real: Ubicado sobre la Rua Don Pedro V cerca al barrio alto, este Jardín de 1,15 hectáreas cuenta con zonas de vegetación, jardines infantiles, zona de alimentación, fuente de agua y mesas bancas para pasar la tarde. Este lugar es punto de encuentro de turistas que tiene corte arquitectónico inglés y fue inaugurado oficialmente en 1915, aunque su historia de construcción se remonta al siglo XVIII. Las grabaciones binaurales fueron realizadas paralelo a la Rua Don Pedro V (P13). 
Chapter Four. Final considerations, Conclusions and Future Work

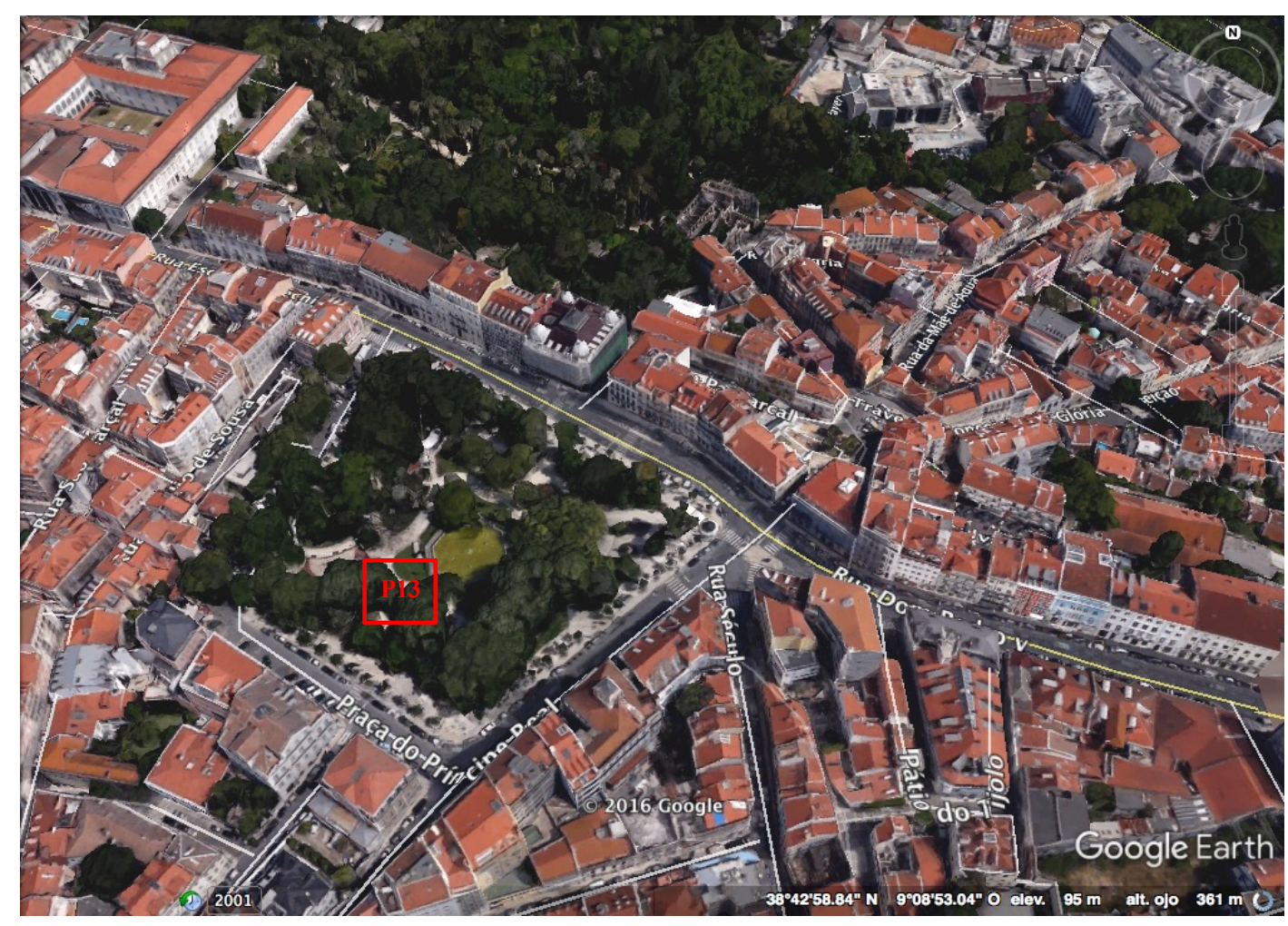


Anexo 6. Herramienta de evaluación in situ en Lisboa.

\begin{tabular}{|l|l|l|l|l|}
\hline & & & \multicolumn{2}{|l|}{ Número de prova: } \\
\hline Data: & & & Local: \\
\hline Hora: & & & Pesquisador: \\
\hline
\end{tabular}

\begin{tabular}{|c|c|c|c|c|c|}
\hline \multicolumn{3}{|c|}{ Qual é a frequência com que visita este parque: } & \multirow[b]{2}{*}{$\begin{array}{l}1 \text { vez por } \\
\text { semana }\end{array}$} & \multirow[b]{2}{*}{$\begin{array}{l}\text { Entre } 2 \text { e } 3 \\
\text { vezes por } \\
\text { semana }\end{array}$} & \multirow[b]{2}{*}{$\begin{array}{l}4 \text { ou mais vezes } \\
\text { por semana }\end{array}$} \\
\hline & $\begin{array}{l}\text { Menos de } \\
\text { uma vez por } \\
\text { mês }\end{array}$ & $\begin{array}{l}\text { Entre } 2 \text { e } 3 \\
\text { vezes por } \\
\text { mês }\end{array}$ & & & \\
\hline & $\square$ & $\square$ & $\square$ & $\square$ & $\square$ \\
\hline \multicolumn{6}{|c|}{ Quanto tempo costuma permanecer no parque? } \\
\hline & & $\begin{array}{l}\text { entre } 10 \text { e } 30 \\
\text { minutos }\end{array}$ & $\begin{array}{l}\text { entre } 30 \text { e } 60 \\
\text { minutos } \\
\text { minutos }\end{array}$ & $\begin{array}{l}\text { entre } 1 \mathrm{e} \\
\text { duas horas }\end{array}$ & $\begin{array}{l}\text { mais de duas } \\
\text { horas }\end{array}$ \\
\hline & & $\square$ & $\square$ & $\square$ & $\square$ \\
\hline \multicolumn{6}{|c|}{ Qual é o motivo de sua visita/estadia: } \\
\hline & Act. Física & $\begin{array}{l}\text { Lugar de } \\
\text { passagem }\end{array}$ & $\begin{array}{l}\text { Contemplaçã } \\
\text { o }\end{array}$ & $\begin{array}{l}\text { Leitura e } \\
\text { meditação }\end{array}$ & Lazer geral \\
\hline & $\square$ & $\square$ & $\square$ & $\square$ & $\square$ \\
\hline & Trabalho & Outro & & & \\
\hline & $\square$ & $\square$ & Qual: & & \\
\hline
\end{tabular}

\begin{tabular}{|c|c|c|c|c|c|}
\hline \multicolumn{6}{|c|}{ Até que ponto você ouve as seguintes fontes sonoras: } \\
\hline & Não escuta & Pouco & Algum & Muito & $\begin{array}{l}\text { Completamente } \\
\text { dominante }\end{array}$ \\
\hline $\begin{array}{l}\text { 1. Tráfego (carros, } \\
\text { comboios, motocicletas, } \\
\text { barcos, aviões, etc.) }\end{array}$ & $\square$ & $\square$ & $\square$ & $\square$ & $\square$ \\
\hline $\begin{array}{l}\text { 2. Sons de máquinas e } \\
\text { equipamento (sistemas } \\
\text { de ventilação, ventoina, }\end{array}$ & $\square$ & $\square$ & $\square$ & $\square$ & $\square$ \\
\hline $\begin{array}{l}\text { 3. Outros sons (industria, } \\
\text { construção, sereias, } \\
\text { maquinaria) }\end{array}$ & $\square$ & $\square$ & $\square$ & $\square$ & $\square$ \\
\hline $\begin{array}{l}\text { (pessoas falando, passos, } \\
\text { crianças jogando, risas, } \\
\text { etc.) }\end{array}$ & $\square$ & $\square$ & $\square$ & $\square$ & $\square$ \\
\hline $\begin{array}{l}\text { 5. Sons naturais } \\
\text { (pássaros, agua, vento, }\end{array}$ & $\square$ & $\square$ & $\square$ & $\square$ & $\square$ \\
\hline \multicolumn{6}{|c|}{ Qual/quais destes sons considera que caracteriza ou identifica este espaço: } \\
\hline \multirow[t]{2}{*}{\begin{tabular}{|l|l}
$\mid$ & \\
& \\
\end{tabular}} & Tráfego & $\begin{array}{l}\text { Sons de } \\
\text { máquinas e } \\
\text { equipamento }\end{array}$ & Outros sons & $\begin{array}{l}\text { Sons } \\
\text { Humanos }\end{array}$ & Sons naturais \\
\hline & $\square$ & $\square$ & $\square$ & $\square$ & $\square$ \\
\hline
\end{tabular}


Chapter Four. Final considerations, Conclusions and Future Work

\begin{tabular}{|c|c|c|c|c|c|c|}
\hline \multicolumn{7}{|c|}{ De forma geral como avalia este ambiente sonoro: } \\
\hline & & Muito bom & Bom & Neutro & Mau & Muito Mau \\
\hline & & $\square$ & $\square$ & $\square$ & $\square$ & $\square$ \\
\hline \multicolumn{7}{|c|}{$\begin{array}{l}\text { Em que extensão considera que os seguintes } 8 \text { fatores descrevem este ambiente sonoro? Indique } \\
\text { uma opção para cada fator: }\end{array}$} \\
\hline \multicolumn{2}{|c|}{ O ambiente sonoro é: } & $\begin{array}{l}\text { completame } \\
\text { nte }\end{array}$ & $\begin{array}{l}\text { Concordo } \\
\text { bastante }\end{array}$ & $\begin{array}{l}\text { concordo } \\
\text { nem discordo }\end{array}$ & $\begin{array}{l}\text { Discordo } \\
\text { bastante }\end{array}$ & $\begin{array}{l}\text { Discordo } \\
\text { completamente }\end{array}$ \\
\hline \multicolumn{2}{|c|}{ Agradável } & $\square$ & ㄱ & $\square$ & 口 & ( \\
\hline \multicolumn{2}{|l|}{ Caótico } & 口 & 口 & $\square$ & 口 & 口 \\
\hline \multicolumn{2}{|c|}{ Excitante/vibrante } & $\square$ & 口 & $\square$ & ○ & ○ \\
\hline \multicolumn{2}{|c|}{ Uniforme } & $\square$ & $\square$ & $\square$ & $\square$ & $\square$ \\
\hline \multicolumn{2}{|l|}{ Calmo } & ○ & 口 & $\square$ & ○ & $\square$ \\
\hline \multicolumn{2}{|l|}{ Desagradáv } & 口 & 口 & $\square$ & $\square$ & $\square$ \\
\hline \multicolumn{2}{|l|}{ Agitado } & ○ & 口 & $\square$ & $\square$ & $\square$ \\
\hline \multicolumn{2}{|l|}{ Monótono } & ב & ב & $\square$ & $\square$ & $\square$ \\
\hline \multicolumn{7}{|c|}{ Em termos de infraestrutura e instalações, como avalia este parque? } \\
\hline & & Muito bom & Bom & Neutro & Mau & Muito Mau \\
\hline & & $\square$ & 马 & $\square$ & ב & $\square$ \\
\hline \multicolumn{7}{|c|}{ Como descreveria de forma geral o ambiente visual : } \\
\hline & & Muito bom & Bom & Neutro & Mau & Muito mau \\
\hline & & ב & ב & 马 & 口 & ב \\
\hline \multicolumn{2}{|c|}{ Ano de nascimento: } & & Sexo: $M()$ & & & \\
\hline \multirow[t]{2}{*}{ Ocupação: } & & Estudante & Trabalhador & Aposentado & Outro & \\
\hline & & $\square$ & a & ᄆ & $\square$ & \\
\hline \multicolumn{3}{|c|}{ Quais são os seus sons preferidos: } & & & & \\
\hline \multicolumn{2}{|c|}{ Local de Moradia: } & & & & & \\
\hline
\end{tabular}


Anexo 7. Herramienta de evaluación en laboratorio en portugués.

\section{Local de gravação: $\mathrm{XXXX}$}

\begin{tabular}{|c|c|c|c|c|c|c|}
\hline \multicolumn{7}{|c|}{ Até que ponto você ouve as seguintes fontes sonoras: } \\
\hline & & Não escuta & Pouco & Algum & Muito & $\begin{array}{l}\text { Completamente } \\
\text { dominante }\end{array}$ \\
\hline \multicolumn{2}{|c|}{$\begin{array}{l}\text { 1. Tráfego (carros, comboios, } \\
\text { motocicletas, barcos, aviões, }\end{array}$} & $\square$ & $\square$ & $\square$ & $\square$ & $\square$ \\
\hline \multicolumn{2}{|c|}{$\begin{array}{l}\text { 2. Sons de motores (sistemas de } \\
\text { ventilação, etc.) }\end{array}$} & $\square$ & $\square$ & $\square$ & $\square$ & $\square$ \\
\hline \multicolumn{2}{|c|}{$\begin{array}{l}\text { 3. Outros sons (indústria, } \\
\text { construção, comercio, sirenes, }\end{array}$} & $\square$ & $\square$ & $\square$ & $\square$ & $\square$ \\
\hline \multicolumn{2}{|c|}{$\begin{array}{l}\text { 4. Sons humanos (pessoas } \\
\text { falando, passos, crianças }\end{array}$} & $\square$ & $\square$ & $\square$ & $\square$ & $\square$ \\
\hline \multicolumn{2}{|c|}{$\begin{array}{l}\text { 5. Sons naturais (pássaros, agua, } \\
\text { vento, etc.) }\end{array}$} & $\square$ & $\square$ & $\square$ & $\square$ & $\square$ \\
\hline \multicolumn{7}{|c|}{ Qual destes sons considera que caracteriza ou identifica esta gravação: } \\
\hline \multirow{2}{*}{$\begin{array}{l}\text { máquinas e } \\
\text { equipamentos } \\
\square\end{array}$} & Trafego & $\begin{array}{l}\text { Sons } \\
\text { naturais }\end{array}$ & $\begin{array}{l}\text { Sons } \\
\text { Humanos }\end{array}$ & $\begin{array}{l}\text { Outros } \\
\text { (quais?) }\end{array}$ & & \\
\hline & $\square$ & $\square$ & $\square$ & $\square$ & & \\
\hline
\end{tabular}

\begin{tabular}{|l|l|l|l|l|l|l|}
\hline \multicolumn{6}{|l|}{ De forma geral como avalia o ambiente sonoro apresentado na gravação?: } \\
\hline Muito bom & Bom & Neutro & Mau & Muito Mau & & \\
\hline$\square$ & $\square$ & $\square$ & $\square$ & $\square$ & \\
\hline
\end{tabular}

\begin{tabular}{|c|c|c|c|c|c|c|}
\hline \multicolumn{7}{|c|}{$\begin{array}{l}\text { Em que extensão considera que os seguintes } 8 \text { fatores descre } \\
\text { Indique uma opção para cada fator: }\end{array}$} \\
\hline \multicolumn{2}{|c|}{ O ambiente sonoro é: } & $\begin{array}{l}\text { completame } \\
\text { nte }\end{array}$ & $\begin{array}{l}\text { Concordo } \\
\text { bastante }\end{array}$ & $\begin{array}{l}\text { concordo } \\
\text { nem }\end{array}$ & $\begin{array}{l}\text { Discordo } \\
\text { bastante }\end{array}$ & $\begin{array}{l}\text { Discordo } \\
\text { completamente }\end{array}$ \\
\hline \multicolumn{2}{|c|}{ Agradável } & $\square$ & $\square$ & $\square$ & $\square$ & $\square$ \\
\hline \multicolumn{2}{|l|}{ Caótico } & $\square$ & $\square$ & $\square$ & $\square$ & $\square$ \\
\hline \multicolumn{2}{|c|}{ Excitante/vibrante } & $\square$ & $\square$ & $\square$ & $\square$ & $\square$ \\
\hline Uniforme & & $\square$ & $\square$ & $\square$ & $\square$ & $\square$ \\
\hline \multicolumn{2}{|l|}{ Calmo } & $\square$ & $\square$ & $\square$ & $\square$ & $\square$ \\
\hline Desagradável & & $\square$ & $\square$ & $\square$ & $\square$ & $\square$ \\
\hline \multicolumn{2}{|l|}{ Agitado } & $\square$ & $\square$ & $\square$ & $\square$ & $\square$ \\
\hline Monótono & & $\square$ & $\square$ & $\square$ & $\square$ & $\square$ \\
\hline \multicolumn{7}{|c|}{ Conhece e/ou tem visitado o local no qual foi feita esta gravação? } \\
\hline Sim & $\square$ & Não & $\square$ & & & \\
\hline \multicolumn{7}{|l|}{ gravação? } \\
\hline $\begin{array}{l}\text { menos de } 10 \\
\text { minutos }\end{array}$ & $\begin{array}{l}\text { entre } 10 \text { e } 30 \\
\text { minutos }\end{array}$ & $\begin{array}{l}\text { entre } 30 \text { e } \\
60 \text { minutos }\end{array}$ & $\begin{array}{l}\text { entre } 1 \text { e } 2 \\
\text { horas }\end{array}$ & $\begin{array}{l}\text { mais de } 2 \\
\text { horas }\end{array}$ & & \\
\hline$\square$ & $\square$ & $\square$ & $\square$ & $\square$ & & \\
\hline \multicolumn{7}{|c|}{ Que uso você daria a um local com um ambiente sonoro como este? } \\
\hline Act. Física & $\begin{array}{l}\text { Lugar de } \\
\text { passagem }\end{array}$ & $\begin{array}{l}\text { Contemplaç } \\
\text { ão }\end{array}$ & $\begin{array}{l}\text { Leitura e } \\
\text { meditação }\end{array}$ & Lazer geral & Trabalho & Outro (qual): \\
\hline$\square$ & $\square$ & $\square$ & $\square$ & $\square$ & $\square$ & $\square$ \\
\hline
\end{tabular}


Chapter Four. Final considerations, Conclusions and Future Work

\section{Anexo 8. Pruebas de normalidad de Shapiro-Wilk para los parques de Lisboa-Portugal.}

Los estadísticos descriptivos más relevantes son presentados en la tabla 1.

Tabla 24-A. Pruebas de normalidad de Shapiro-Wilk

\begin{tabular}{|c|c|c|c|c|c|c|c|}
\hline & & Shapiro-Wilk in situ & & & Shapiro-Wilk Lab & & \\
\hline Place & Perceptual atributte & Statistic & df & Sig. & Statistic & df & Sig. \\
\hline \multirow{9}{*}{ LJFG } & General Assessment & 0,744 & 20 & 0 & 0,795 & 25 & 0 \\
\hline & Pleasant & 0,608 & 20 & 0 & 0,821 & 25 & 0,001 \\
\hline & Chaotic & 0,351 & 20 & 0 & 0,832 & 25 & 0,001 \\
\hline & Exciting & 0,796 & 20 & 0,001 & 0,836 & 25 & 0,001 \\
\hline & Uneventful & 0,792 & 20 & 0,001 & 0,829 & 25 & 0,001 \\
\hline & Calm & 0,759 & 20 & 0 & 0,762 & 25 & 0 \\
\hline & Annoying & 0,447 & 20 & 0 & 0,832 & 25 & 0,001 \\
\hline & Eventful & 0,743 & 20 & 0 & 0,873 & 25 & 0,005 \\
\hline & Monotonous & 0,822 & 20 & 0,002 & 0,714 & 25 & 0 \\
\hline \multirow{9}{*}{ LJE } & General Assessment & 0,488 & 20 & 0 & 0,52 & 25 & 0 \\
\hline & Pleasant & 0,728 & 20 & 0 & 0,521 & 25 & 0 \\
\hline & Chaotic & 0,699 & 20 & 0 & 0,901 & 25 & 0,019 \\
\hline & Exciting & 0,846 & 20 & 0,005 & 0,884 & 25 & 0,008 \\
\hline & Uneventful & 0,893 & 20 & 0,031 & 0,852 & 25 & 0,002 \\
\hline & Calm & 0,71 & 20 & 0 & 0,847 & 25 & 0,002 \\
\hline & Annoying & 0,537 & 20 & 0 & 0,776 & 25 & 0 \\
\hline & Eventful & 0,85 & 20 & 0,005 & 0,697 & 25 & 0 \\
\hline & Monotonous & 0,816 & 20 & 0,002 & 0,744 & 25 & 0 \\
\hline \multirow{9}{*}{ LJPR } & General Assessment & 0,671 & 20 & 0 & 0,721 & 25 & 0 \\
\hline & Pleasant & 0,79 & 20 & 0,001 & 0,858 & 25 & 0,003 \\
\hline & Chaotic & 0,74 & 20 & 0 & 0,838 & 25 & 0,001 \\
\hline & Exciting & 0,817 & 20 & 0,002 & 0,86 & 25 & 0,003 \\
\hline & Uneventful & 0,748 & 20 & 0 & 0,894 & 25 & 0,014 \\
\hline & Calm & 0,759 & 20 & 0 & 0,771 & 25 & 0 \\
\hline & Annoying & 0,623 & 20 & 0 & 0,8 & 25 & 0 \\
\hline & Eventful & 0,879 & 20 & 0,017 & 0,874 & 25 & 0,005 \\
\hline & Monotonous & 0,788 & 20 & 0,001 & 0,751 & 25 & 0 \\
\hline
\end{tabular}


Según los resultados presentados en la tabla 3, puede observarse que todos los valores $p$ de significancia son menores a 0,05 , por lo que no es posible afirmar que los diferentes atributos perceputuales en estudio presentan distribución normal para las metodologías in situ y en laboratorio tomando un valor de significancia del $5 \%$. 